\title{
Spatial models of metapopulations and benthic communities in patchy environments
}

by

Mark Forrest Hill

\author{
M.S. Biology \\ University of Massachusetts, Boston 1995 \\ Submitted to the department of biology in partial fulfillment \\ of the requirements for the degree of \\ Doctor of Philosophy in Biological Oceanography \\ at the \\ MASSACHUSETTS INSTITUTE OF TECHNOLOGY \\ and \\ WOODS HOLE OCEANOGRAPHIC INSTITUTION
}

SEPTEMBER 2000

(c)Mark Forrest Hill. All rights reserved.

The author hereby grants to MIT and WHOI permission to reproduce and to distribute copies of this thesis in whole or in part.

Author

Joint Program in Oceanography and Applied Ocean Science and Engineering, Massachusetts Institute of Technology/Woods Hole Oceanographic Institution

Certified by

Dr. Hal Caswell

Senior Scientist, WHOI

Thesis Supervisor

Accepted by

Dr. Mark E. Hahn Chairman, MIT/WHOI Joint Committee on Biological Oceanography 


\title{
Spatial models of metapopulations and benthic communities in patchy environments
}

\author{
by \\ Mark Forrest Hill \\ Submitted in partial fulfillment \\ of the requirements for the degree of \\ Doctor of Philosophy \\ at the \\ MASSACHUSETTS INSTITUTE OF TECHNOLOGY \\ and \\ WOODS HOLE OCEANOGRAPHIC INSTITUTION \\ September 2000
}

\begin{abstract}
The distribution of organisms in space has important consequences for the function and structure of ecological systems. Such distributions are often referred to as patchy, and a patch-based approach to modeling ecosystem dynamics has become a major research focus. These models have been used to explore a wide range of questions concerning population, metapopulation, community, and landscape ecology, in both terrestrial and aquatic systems.

In this dissertation I develop and analyze a series of spatial models to study the dynamics of metapopulations and marine benthic communities in patchy environments. All the models have the form of a discrete-time Markov chain, and assume that the landscape is composed of discrete patches, each of which is in one of a number of possible states. The state of a patch is determined by the presence of an individual of a given species, a local population, or a group of species, depending on the spatial scale of the model.

The research is organized into two main parts as follows. In the first part, I present an analysis of the effects of habitat destruction on metapopulation persistence. Theoretical studies have already shown that a metapopulation goes extinct when the fraction of suitable patches in the landscape falls below a critical threshold (the so called extinction threshold). This result has become a paradigm in conservation biology and several models have been developed to calculate extinction thresholds for endangered species. These models, however, generally do not take into account the spatial arrangement of habitat destruction, or the actual size of the landscape. To investigate how the spatial structure of habitat destruction affects persistence, I compare the behavior of two models: a spatially implicit patch-occupancy model (which recreates the extinction patterns found in other models) and a spatially explicit cellular automaton (CA) model. In the CA, I use fractal arrangements of suitable and unsuitable patches to simulate habitat destruction and show that the extinction threshold depends on the fractal dimension of the landscape. To investigate how
\end{abstract}


habitat destruction affects persistence in finite landscapes, I develop and analyze a chain-binomial metapopulation (CBM) model. This model predicts the expected extinction time of a metapopulation as a function of the number of patches in the landscape and the number of those patches that are suitable for the population. The CBM model shows that the expected time to extinction decreases greater than exponentially as suitable patches are destroyed. I also describe a statistical method for estimating parameters for the CBM model in order to evaluate metapopulation viability in real landscapes.

In the second part, I develop and analyze a series of Markov chain models for a rocky subtidal community in the Gulf of Maine. Data for the model comes from ten permanent quadrats (located on Ammen Rock Pinnacle at 30 meters depth) monitored over an 8-year period (1986-1994). I first parameterize a linear (homogenous) Markov chain model from the data set and analyze it using an array of novel techniques, including a compression algorithm to classify species into functional groups, a set of measures from stochastic process theory to characterize successional patterns, sensitivity analyses to predict how changes in various ecological processes effect community composition, and a method for simulating species removal to identify keystone species. I then explore the effects of time and space on successional patterns using log-linear analysis, and show that transition probabilities vary significantly across small spatial scales and over yearly time intervals. I examine the implications of these findings for predicting equilibrium species abundances and for characterizing the transient dynamics of the community. Finally, I develop a nonlinear Markov chain for the rocky subtidal community. The model is parameterized using maximum likelihood methods to estimate density-dependent transition probabilities. I analyze the best fitting models to study the effects of nonlinear species interactions on community dynamics, and to identify multiple stable states in the subtidal system.

Dissertation Advisor: Hal Caswell

Title: Senior Scientist, Woods Hole Oceanographic Institution 


\section{Acknowledgements}

I owe a large debt of gratitude to my advisor Hal Caswell, for his patience, guidance and support, and for giving me the freedom to explore a range of different research avenues. I am also extremely grateful to Jon Witman for sharing his data with me, for his valuable counsel on the ecology of rocky subtidal communities, and for many fruitful hours of discussion over several strong cups of coffee. I would also like to thank Mike Nubert for his friendship, for helping me expand my quantitative skills and for showing me the beauty of mathematical ecology. I am also grateful to the other members of my committee, Glen Flierl and Jesus Pineda, for their insights and advise. Finally, I would like to thank Joel Cohen for his advise on the use of stochastic matrices.

A number of graduate students, poctdocs, technicians and scientists have made my time here at WHOI a more rewarding experience, through their support and camaraderie. I would especially like to thank Michael Atkins, Shannon Bard, Katie Boissonneault-Cellineri, Anne Canaday, Alvin Gomez, Rebecca Green, Robert Hamersley, Patrick Miller, Nicole Poulton, Mario Sengco, Linda Traykovski, Andreas Teske, Rebecca Thomas, Sandra Werner, and Bruce Woodin for many hours of laughs and the occasional outbursts of insanity. I would also like to thank Mary Jane Tucci and Julie Westwater for helping me navigate the MIT/WHOI labyrinth of paper work. Finally, I would like to extent a special thanks to Amelie and Rudy Scheltema for being good neighbors and for their willingness to accept a modeler as a colleague and friend.

I am particularly grateful to my wife, Angela Tymrak, whose love and encouragement provided me with the confidence I needed to undertake and finish this dissertation. For her support I am eternally thankful.

This work was supported by the Office of Naval Research and the National Science Foundation through the following grants to Hal Caswell: ONR-URIP Grant N0001492-J-1527, NSF Grants DEB-9119420, DEB-95-27400, OCE-981267 and OCE-9302238. 


\section{Contents}

1 Introduction $\quad 13$

1.1 Characterizing patch dynamics: A discrete-time Markov chain approach 16

1.2 Thesis structure . . . . . . . . . . . . . . . . 17

1.2 .1 Chapter details ..................... 19

1.3 References ......................... 22

2 Habitat fragmentation and extinction thresholds on fractal land$\begin{array}{ll}\text { scapes } & 29\end{array}$

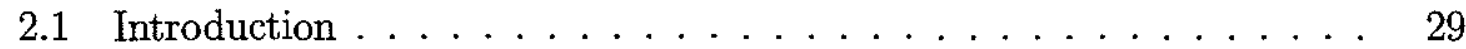

2.1.1 Lande's Model .................. 31

2.1.2 Metapopulation Models .............. 32

2.2 The Patch-Occupancy Model . . . . . . . . . . . . 33

2.2.1 Stability Analysis and Extinction Threshold . . . . . . . 36

2.3 Adding Landscape Structure . . . . . . . . . . . . . . . . . . 39

2.3.1 Fractal Landscapes . . . . . . . . . . . . . . 40

2.4 Simulation Methods . . . . . . . . . . . . . . . . 43

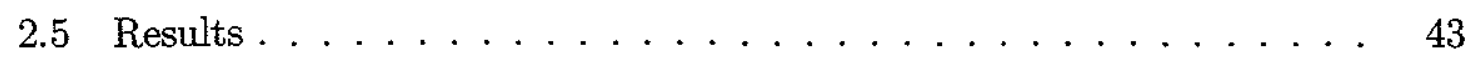

2.6 Discussion .......................... 46

2.7 References ........................... 48 
3 The effects of habitat destruction in finite landscapes: A chainbinomial metapopulation model $\quad \mathbf{5 2}$

3.1 Introduction . . . . . . . . . . . . . . 52

3.2 Model Description . . . . . . . . . . . . . . . 54

3.2 .1 Extinction matrix .............. 55

3.2.2 Colonization matrix . . . . . . . . . . . . 55

3.2.3 CBM Model . . . . . . . . . . . . . . . . . 57

3.2 .4 Search Ability . . . . . . . . . . . . . 59

3.2.5 Model Analysis . . . . . . . . . . . . . . . . 60

3.3 Results: The Effect of Habitat Destruction . . . . . . . . . . . . 64

3.4 Parameter Estimation . . . . . . . . . . . . . 67

3.4.1 Likelihood function . . . . . . . . . . . . . 69

3.4 .2 An Example . . . . . . . . . . . . . . . 70

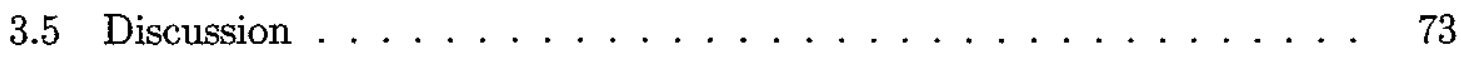

3.6 References . . . . . . . . . . . . . . 76

4 A Markov chain model of a rocky subtidal community: succession and species interactions in a complex assemblage $\quad 79$

4.1 Introduction . . . . . . . . . . . . . . . . 79

4.2 Background: Rocky Subtidal Communities . . . . . . . . . . . . 82

4.3 Model Structure and Analysis . . . . . . . . . . . . . . . 84

4.3.1 Parameter estimation . . . . . . . . . . . . 87

4.3 .2 Identifying Functional Groups . . . . . . . . . . . . . 88

4.3.3 Rates and patterns of succession . . . . . . . . . . 90

4.3 .4 Sensitivity analysis ................... 92

4.3.5 Effects of species removals .............. 94

4.4 Results .............................. 95

4.4.1 Equilibrium distribution ................ 98 
4.4.2 Quantifying functional relatedness and combining species into functional groups . . . . . . . . . . . . . . . . 99

4.4.3 Characterizing patch dynamics . . . . . . . . . . 102

4.4 .4 Sensitivity Analysis . . . . . . . . . . . . . . 105

4.4 .5 Species removal effects . . . . . . . . . . . . . . 108

4.5 Discussion . . . . . . . . . . . . . . . . 111

4.6 References . . . . . . . . . . . . . . . . . . . . . . . . . 119

5 Temporal and spatial variation in successional patterns in a rocky subtidal community: Statistical methods, Markov chain models, and

$\begin{array}{ll}\text { Species distributions } & 128\end{array}$

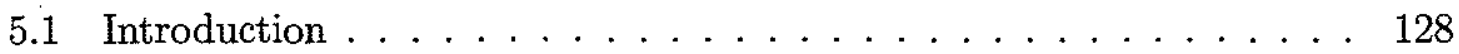

5.2 Methods . . . . . . . . . . . . . . . . . 130

5.2 .1 Data collection ... . . . . . . . . . . . . . . 130

5.2 .2 Statistical Analysis . . . . . . . . . . . . . . . . . 132

5.3 Results: The effects of time and location on patch succession . . . . 134

5.4 Markov chain models: Species composition and community dynamics 134

5.4.1 Equilibrium Predictions . . . . . . . . . . . . . 136

5.4 .2 Community Dynamics . . . . . . . . . . . . . . . 138

5.5 Temporal and spatial variability in species distributions . . . . . . 144

5.6 Discussion . . . . . . . . . . . . . . . . . . . . . 145

5.7 References . . . . . . . . . . . . . . . . . . . . . . 149

6 A Nonlinear Markov Chain of a Rocky Subtidal Community: Quantifying the effects of species interactions on community dynamics 155

6.1 Introduction . . . . . . . . . . . . . . . . . 155

6.2 Data collection . . . . . . . . . . . . . . . 158

6.3 A nonlinear Markov chain model . . . . . . . . . . . . . 160 
6.4 Methods . . . . . . . . . . . . . . . . . . . . . . . . 162

6.4 .1 Parameter estimation . . . . . . . . . . . . . . . 162

6.4 .2 Set of candidate models . . . . . . . . . . . . . 163

6.4.3 Model Fitting using a Two Step Method . . . . . . . . . 165

6.4 .4 Best fitting model . . . . . . . . . . . . . . . 167

6.5 Results: Nonlinear Dynamics of the Subtidal Community . . . . . . 167

6.5 .1 Numerical simulations . . . . . . . . . . . . . . . . . 167

6.5 .2 Equilibrium dynamics . . . . . . . . . . . . . . . 169

6.5 .3 Convergence times . . . . . . . . . . . . . . 171

6.6 Dependence of species interactions on neighborhood size $\ldots \ldots . .172$

6.6 .1 Equilibrium distributions . . . . . . . . . . . . . . . 173

6.6.2 Distribution of species interaction coefficients $\ldots \ldots \ldots .175$

6.6.3 Convergence times and patterns of succession . . . . . . 177

6.6 .4 Rates of local succession . . . . . . . . . . . . . . 179

6.7 Bifurcation Analysis . . . . . . . . . . . . . . . . 180

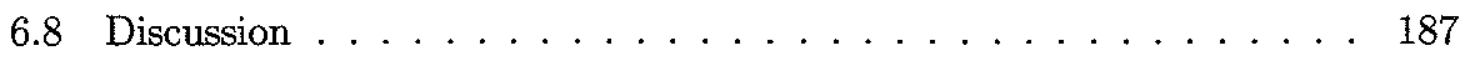

6.9 Conclusion . . . . . . . . . . . . . . . . . . . 193

7 Conclusion and Future Directions $\quad 206$

7.1 New insights into the effects of habitat destruction . . . . . . . . 207

7.2 Markov chain models of sessile communities . . . . . . . 210

7.3 Future research directions . . . . . . . . . . . . . . . . 213

7.3.1 Metapopulations models: Habitat restoration and Marine reserves213

7.3.2 Spatially explicit models of benthic communities . . . . . . 217

7.4 References . . . . . . . . . . . . . . . . . . . . 219 


\section{List of Figures}

1.1 Transition diagram for a four state Markov chain model. . . . . . . . 18

2.1 Transition diagram for the patch-occupancy model. . . . . . . . . 35

2.2 A one-dimensional map for the patch-occupancy model. . . . . . . . . 38

2.3 Equilibrium frequency of occupied patches. . . . . . . . . . . 39

2.4 Browian fractal landscapes . . . . . . . . . . . . . . . . 42

2.5 Equilibrium frequencies for the $\mathrm{CA}$ on fractal landscapes. . . . . . . 44

2.6 Extinction thresholds on random and fractal landscapes. . . . . . . 45

3.1 Example transition matrices for the chain-binomial model. . . . . . . 58

3.2 Stochastic realization of the CBM model. . . . . . . . . . . . . 61

3.3 Time series of the distribution of landscapes. . . . . . . . . . . . . 62

3.4 Quasi-equilibrium frequency as a function of the number of suitable

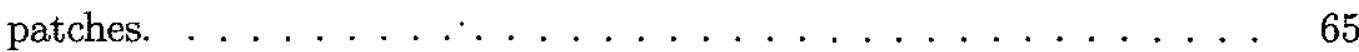

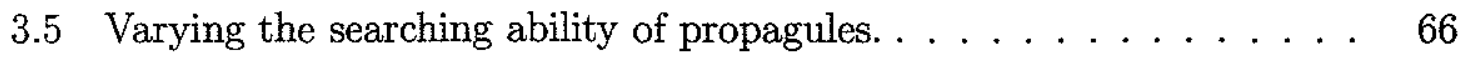

3.6 The effect of habitat destruction on extinction times. . . . . . . . 67

3.7 Mean persistence probability as a function of time. . . . . . . 68

3.8 Patch occupancy data for Meltiaea cinxia and Cotesia melitaearum . 71

3.9 The expected extinction time of Meltiaea cinxia . . . . . . . . . 73

4.1 A photo quadrat of the subtidal community. . . . . . . . . . 85

4.2 Method of collecting data from quadrat photos. . . . . . . . . 87

4.3 Predicted and observed species abundances. . . . . . . . . . . . 98 
4.4 Distance dendogram of species states. . . . . . . . . . . 100

4.5 Percent change in equilibrium distribution. . . . . . . . . . . 101

4.6 Smoluchowski recurrence times. . . . . . . . . . . . . . . . . . . 104

4.7 First passage times. . . . . . . . . . . . . . . . 106

4.8 Sensitivity of community diversity $(H)$ to changes in $a_{i j} \ldots \ldots \ldots$

4.9 Predicted change in species evenness diversity. . . . . . . . . . . 109

4.10 Predicted change in community resilience. . . . . . . . . . 110

4.11 The percent change in species abundance. . . . . . . . . . 117

5.1 The effects of time (T) and location (L) on transition probabilities. . 135

5.2 Predicted vs. observed species distributions. . . . . . . . . . . 139

5.3 Simulations of the non-homogeneous Markov chain. . . . . . . . . . 141

5.4 Distribution of dominance times for Hymedesmia 1 and Crisia. . . . . 142

5.5 Comparison of equilibrium distributions between the homogeneous and non-homogenous Markov chains . . . . . . . . . . . . . . . 143

5.6 Temporal and spatial variability of relative species abundances. . . 146

6.1 Coexisting attractors for the best fitting nonlinear Markov chain . . 170

6.2 Observed species abundances in the Gulf of Maine . . . . . . . . . 171

6.3 Coexisting attractors at different neighborhood sizes. . . . . . . . . 174

6.4 Distribution of interaction coefficients. . . . . . . . . . 176

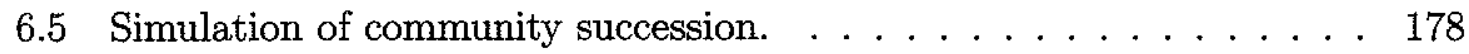

6.6 Smouchowski recurrence times at equilibrium . . . . . . . . . . 181

6.7 Interaction strength bifurcation diagrams. . . . . . . . . . . . 184

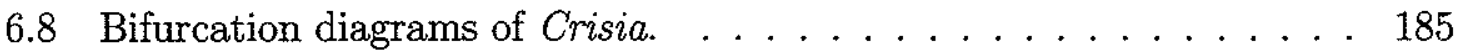

6.9 Sensitivity to initial conditions. . . . . . . . . . . . 186

6.10 Bifurcation diagrams of Hymedesmia. . . . . . . . . . . . 188

7.1 The effect of the fractal dimension on equilibrium frequencies and the extinction threshold . . . . . . . . . . . . . . . . . . . 208 
7.2 Metapopulation model of habitat destruction and restoration. . . . . 214

7.3 Hyper-population model of a marine reserve. . . . . . . . . . . . 215 


\section{List of Tables}

3.1 Likelihood estimates of $\beta$ and $\delta \ldots \ldots \ldots \ldots \ldots \ldots \ldots \ldots$

4.1 Subtidal species in the Gulf of Maine data set. . . . . . . . . . 84

4.2 Transition matrix for the subtidal community. . . . . . . . . 96

4.3 Functional group species. . . . . . . . . . . . . . . . . . . . . 102

4.4 Compressed Transition matrix for the subtidal community. . . . . . . 103

4.5 Distribution of eigenvector sensitivities. . . . . . . . . . . . 108

4.6 Summary of matrix entropy $E$ values. . . . . . . . . . . . 113

5.1 Subtidal species in the Gulf of Maine. . . . . . . . . . . . 131

5.2 Product-moment correlation coefficients $r_{p}$ for the temporally-varying Markov chain. . . . . . . . . . . . . . . . . . . . . . . 142

6.1 Invertebrate species identified in the photo quadrat data set. . . . 158

6.2 Species groups used to develop the nonlinear Markov chain. . . . . . 159

6.3 Schematic representation of the transition matrix for the nonlinear Markov chain. . . . . . . . . . . . . . . . . 160

6.4 Summary of the models fitted to the subtidal data . . . . . . . . 164

6.5 The models fitted to the photo quadrat data. . . . . . . . . . . 168

6.6 Best fitting models using smaller neighborhood sizes. . . . . . . . . 173

6.7 Distance between the predicted and observed equilibrium distribution. 175

6.8 Convergence time to equilibrium. . . . . . . . . . . . 177

6.9 Mean turnover rate of patches. . . . . . . . . . . . . 180 


\section{Chapter 1}

\section{Introduction}

Here is the world, sound as a nut, perfect, not the smallest piece of chaos left, never a stitch nor an end, not a mark of haste, or botching, or second thought; but the theory of the world is a thing of shreds and patches.

-Ralph Waldo Emerson

A characteristic feature of the spatial distribution of most species, across a range of spatial scales, is patchiness (Taylor 1961, Taylor and Taylor 1969; Hanski 1994; Wiens 1997). Patchiness refers to the non-homogenous distribution of organisms (and their resources) in space and time (Hanski 1999). At smaller spatial scales, patchiness is generally a consequence of neighborhood effects; i.e., organisms are more likely to interact with their neighbors than with more distant organisms. This is especially true for benthic invertebrates such as corals, sponges, bryozoans, and other sessile marine organisms (Tanner et al. 1994, Sebens 1986, Witman and Dayton 2000). At larger spatial scales, patchiness arises due to dispersal and recruitment patterns (e.g. Horn and MacArthur 1972, Levin 1974, Levin et at. 1984, Paine 1984, Cohen and Levin 1991, Caswell and Cohen 1991a,b, Hanski 1999), physical disturbance (Dayton et al 1970, Witman 1987, Witman and Dayton 2000), and variations in the quality of the environment (Kareiva 1990; Levin 1992; Tilman and Kareiva 1997). Because the absence of a species from a locality may reflect dispersal limitations, unsuitable 
environmental conditions, or competitive exclusion through local biotic interactions, patchy distributions are an emergent property of ecological processes acting over a wide range of spatial scales (e.g., Horn 1971, Hastings 1980, Connell 1985, Gaines and Roughgarden 1985, Menge and Sutherland 1987, Grosberg and Levitan 1992, Connolly and Roughgarden 1998).

The importance of spatial patchiness is increasingly recognized as having important consequences for the function and structure of terrestrial and aquatic systems (Levin and Paine 1974; Steel 1978; Pickett and White 1985; Caswell and Cohen 1991a,b; Weins et. al. 1993; Wu and Levin 1994; Wu and Loucks 1995; Hanski 1999). A patch is generally defined in terms of a spatial unit within a landscape that is different in some physical or biological aspect from its immediate surroundings (Kotliar and Weins 1990). Definitions of a patch include a bounded region within a homogenous background consisting of either single or multiple biological components (Levins and Paine 1974); a spatial location within an environment where the abundance of a resource, a population, or a community of organisms is high (Roughgarden 1977); a location containing an aggregated collection of prey species within which a predator forages (Stephens and Kerbs 1986); territorial sites of individuals (Lande 1987); discrete regions in a landscape containing local populations connected by dispersal (Hanski 1994); and any division or heterogeneity in the abundance of resources (Antolin and Addicott 1991). Thus what is considered a patch depends both on the spatial and temporal scales of interest and the fundamental units of the system being studied (e.g., vertical nutrient distributions at the sediment water interface, territories of spotted owls in fragmented forests, aggregates of benthic organisms, the global distribution of plankton). Spatial patchiness at any scale, however, can be defined in terms of both the composition of patches (i.e. types of patches and their relative abundance) and the spatial distribution of patches (i.e. patch size, shape, and their location in space).

A patch-based approach to studying ecosystem dynamics has become a major 
research focus, and several theoretical methods have been developed to model patch dynamics (Levins 1969; Lande 1989; Caswell and Cohen 1991a,b, 1995; Levin 1992; Weins et. al. 1993; Hanski 1994; Wu and Loucks 1995; Barradas et al. 1996; Caswell and Etter 1999). They include metapopulation models (Levin 1969, Nee and May 1992; Hanski 1999), patch-occupancy models (Caswell and Cohen 1991a,b, 1995; Barradas et al. 1996), reaction-diffusion networks (Karlin and McGregor 1972; Levin 1974; Hastings 1983), Markov chains (Horn 1975; Usher 1979; Tanner et al. 1994,1996), coupled map lattices (Comins et al. 1992; Sole and Valls 1991; Bevers and Flather 1999), interacting particle systems (Mackay and Jan 1994; Sutherland and Jacobs 1994; Durrett and Levin 1994), and cellular automata (Sivlertown et al. 1992; Caswell and Etter 1993, 1999; Molofsky 1994; Dythan 1995; Bascompte and Sole 1996). These modeling approaches have been developed to answer a wide range of questions concerning population ecology, metapopulation dynamics, community ecology, biogeography, and landscape ecology (Wu and Loucks 1995).

In this dissertation I develop a series of models to study the effects of habitat destruction on metapopulation persistence, and to analyze the dynamics of marine benthic communities. The general methods I use to model these systems all have the form of a discrete-time Markov chain. The models assume that the landscape is composed of discrete patches, each of which is in one of a number of possible states. The state of a patch is determined by the presence of an individual of a given species, a local population, or a group of species, depending on the spatial scale of the model. Before describing the specific research presented in the coming chapters, I briefly review the Markov chain approach used to model biological systems in patchy environments. 


\subsection{Characterizing patch dynamics: A discrete- time Markov chain approach}

Consider a landscape composed of patches, each of which is in one of $N$ possible states. Let $x_{i}(t)$ be the proportion of patches in state $i$ at time $t$. The temporal dynamics of the patch states in the landscape can be modeled as a system of difference equations

$$
\begin{aligned}
x_{1}(t+1) & =f_{1}\left(x_{1}(t), x_{2}(t), \ldots, x_{N}(t)\right) \\
x_{2}(t+1) & =f_{2}\left(x_{1}(t), x_{2}(t), \ldots, x_{N}(t)\right) \\
& \vdots \\
x_{N}(t+1) & =f_{N}\left(x_{1}(t), x_{2}(t), \ldots, x_{N}(t)\right)
\end{aligned}
$$

where the functions $f_{i}$ specify the change in the proportion of patches in state $i$ in the interval $(t, t+1)$. If we define a vector $\mathbf{x}(\mathrm{t})$ whose $i$ th element is $x_{i}(t)$, then equation 1.1 can be written in matrix form

$$
\mathbf{x}(t+1)=\mathbf{A x}(t)
$$

where $\mathbf{A}$ is a Markov chain transition matrix whose $(i, j)$ element $\left(a_{i j}\right)$ gives the probability that a patch in state $j$ changes to state $i$ in one time interval. The matrix $\mathbf{A}$ is nonnegative (all $a_{i j} \geq 0$ ) and has the property that each column sums to 1 (i.e. $\mathbf{A}$ is column-stochastic).

The set of possible state transitions for a given biological system can be depicted graphically using a transition diagram (Caswell and Cohen 1991a,b). Figure 1.1 shows an example of a transition diagram for a landscape in which there are four possible patch states. The arrows show the possible transitions among the patch states, while the expressions above the arrows are the probabilities that the transitions occur in the time interval $(t, t+1)$. Transition probabilities can be constant (e.g., $\delta)$, a function 
of time (e.g., $\gamma(t))$, and/or dependent on the frequency of patches in a particular state (e.g., $C_{1}\left(x_{2}\right)$ ). To construct a transition matrix from the diagram simply set the elements $a_{i j}$ equal to the expression on the arrow going from $X_{j}$ to $X_{i}$. The transition matrix corresponding to Figure 1.1 is

$$
\mathbf{A}=\left(\begin{array}{cccc}
1-C_{1}\left(x_{2}\right) & \delta & 0 & \sigma \\
C_{1}\left(x_{1}\right) & \left\{1-C_{2}\left(x_{3}\right)\right\}\{1-\delta\} & 0 & 0 \\
0 & C_{2}\left(x_{3}\right)\{1-\delta\} & 1-\gamma(t) & 0 \\
0 & 0 & \gamma(t) & 1-\sigma
\end{array}\right) .
$$

\subsection{Thesis structure}

The research presented in the following chapters focuses on the use of Markov chain models to investigate patch dynamics at the level of the metapopulation and at the scale of the community. While these models are applicable to many groups of organisms, they are primarily motivated by marine benthic systems.

The models of metapopulation dynamics focus on the effects of habitat destruction on persistence. Theoretical models have shown that a metapopulation cannot persist when the fraction of suitable patches in the landscape falls below a critical threshold (Lande 1987; Nee and May 1992; Lamerson et al. 1994; Kareiva and Wennergren 1995; Noon and McKelvey 1996). Consequently, identifying extinction thresholds for endangered species has become an important paradigm in conservation biology. These studies, however, have mostly ignored two fundamental factors affecting metapopulation persistence: (1). the spatial arrangement of habitat destruction, and (2). the actual size of the patch network. In the first part of the thesis (Chapters 2 and 3) I develop and analyze a set of models that examine how changes in the habitat destruction pattern and changes in the size of the patch network affect predictions about metapopulation persistence and extinction thresholds in fragmented habitats. 


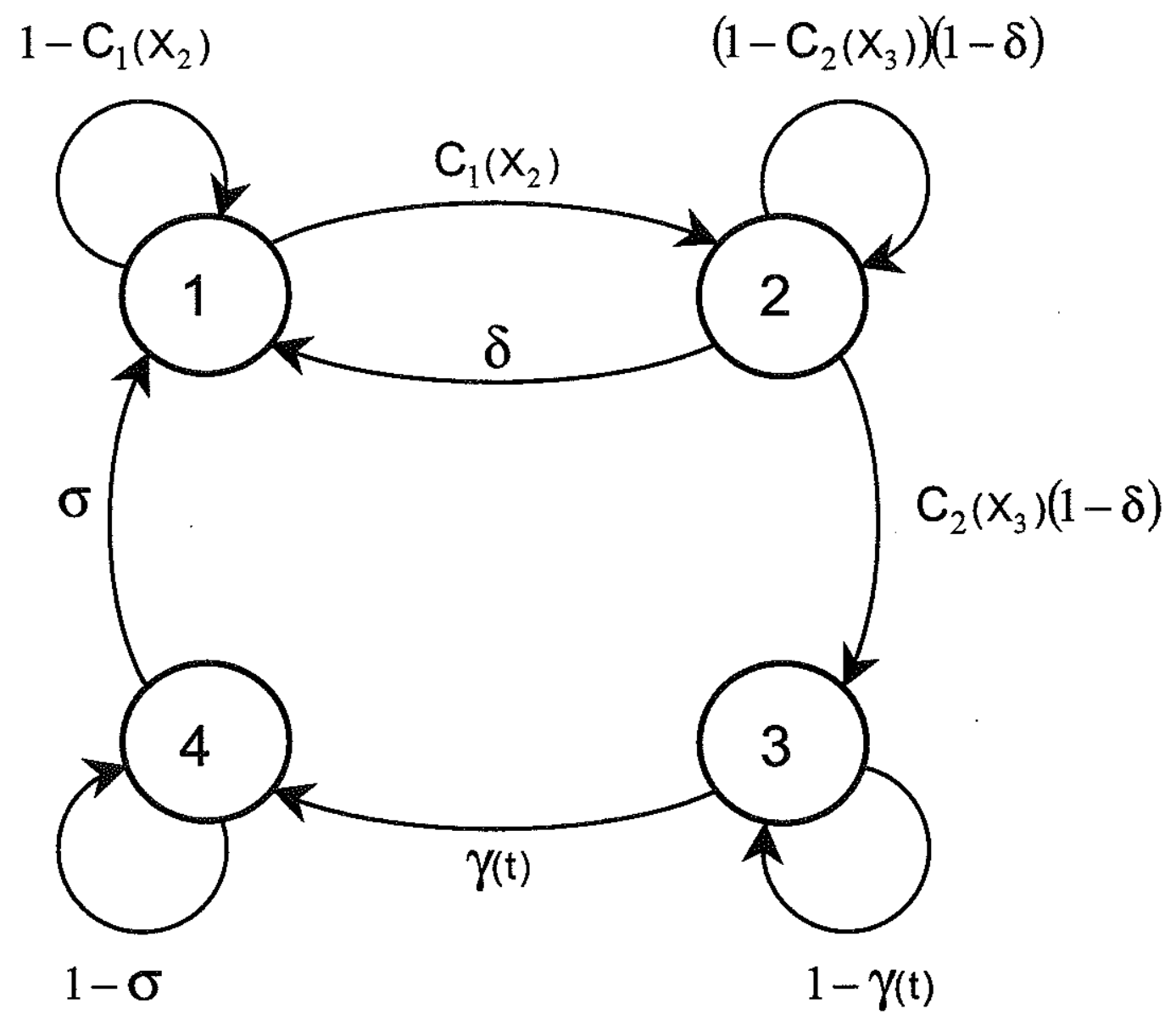

Figure 1.1: Transition diagram for a four state Markov chain model. Each circle represents a possible state of a patch, and in this example the states and are identified as 12,3 , and 4 . The state of a patch is defined by the presence and/or absence of a particular species or species group. For instance, if the diagram were for a predatorprey model then $1=$ empty patches, $2=$ prey only, $3=$ prey and predators, and $4=$ predators only. The arrows connecting the circles represent possible state transitions, which the coefficients above the arrows are the transition probabilities during the time period $(t, t+1)$. 
In second part of the thesis (Chapters 4, 5 and 6), I focus on the construction and analysis of a series of Markov chain models for a rocky subtidal community in the Gulf of Maine. The purpose of this research is four-fold. First, it extends the use of Markov chain models as a tool for marine community ecology. Second, it characterizes how temporal and spatial variation in successional processes affects species abundances and community dynamics. Third, it provides the first evaluation of nonlinear Markov models developed from empirical data. Finally, it increases our understanding of the ecology of sessile invertebrate communities in the rocky subtidal zone, a vast habitat that faces an increasing pace of anthropomorphic disturbance.

\subsubsection{Chapter details}

The chapters in this thesis are organized as follows:

- In chapter 2 I explore the effect of habitat fragmentation when the fragmentation follows a fractal pattern. The goals are to determine the habitat fragmentation pattern affects metapopulation abundance and the amount of habitat destruction the metapopulation can tolerate before it goes extinct. To study these effects, I compare the behavior of two models: a spatially implicit patchoccupancy (PO) model and a spatially explicit cellular automaton (CA) model. The PO model is a nonlinear Markov chain that describes patch dynamics within a fragmented habitat, in which patches are either suitable (i.e. can support local population growth) or unsuitable. Suitable patches are either occupied or unoccupied, and change state depending on rates of colonization and local extinction. The PO model recreates the extinction patterns found in other metapopulation models (e.g., Lande 1987, Hanski 1999). The advantage of the PO model is that it can be directly translated into a stochastic CA model (Caswell and Etter 1993, 1999), in which the state and the location of patches are followed explicitly through time. By using fractal arrangements of suitable 
and unsuitable patches to simulate habitat fragmentation, I show that landscape structure plays a vital role in determining the effects of habitat destruction on persistence.

- In chapter 3 I present a stochastic model for metapopulations in landscapes with a finite but arbitrary number of patches. The model, similar in form to chain-binomial epidemic models, is an absorbing Markov chain that describes the changes in the number of occupied patches as a sequence of binomial probabilities. It predicts the quasi-equilibrium distribution of occupied patches, the expected extinction time, and the probability of persistence to a given age as a function of the number of patches in the landscape and the number of those patches that are suitable for the population. In this chapter I also describe a statistical method for estimating model parameters from time series data in order to evaluate metapopulation viability in real landscapes. An example is presented using published data on the Glanville fritillary butterfly Meltiaea cinxia and its specialist parasitoid Cotesia melitaearum in which the expected extinction time of $M$. cinxia is calculated as a function of the frequency of parasite outbreaks.

- In chapter 4 I describe the development of a homogenous Markov chain model for a rocky subtidal community in the Gulf of Maine. Data for the model comes from ten permanent quadrats (located on Ammen Rock Pinnacle at 30 meters depth) monitored over an 8-year period (1986-1994). I analyze this model using an array of novel techniques, including a compression algorithm for transition matrices to classify species into functional groups, a set of tools from stochastic process theory to characterize successional patterns, and sensitivity analyses to quantify the importance of various ecological processes for maintaining species diversity.

I also present methods for quantifying the effects of species removal on species 
diversity and community resilience. These methods are Markov chain analogs to species removal experiments conducted in natural systems. They provide a theoretical means for classifying the relative importance of individual species to the structure and stability of a community when experimental manipulations are not possible.

- In chapter $5 \mathrm{I}$ explore the effects of time and space on successional patterns in a rocky subtidal community. Using log-linear analysis, I show that transition probabilities vary significantly across small spatial scales and over yearly time intervals. The implications of these findings for predicting equilibrium species abundances and understanding biological processes that drive transient dynamics are discussed. I also use a set of methods introduced by Cohen et al. (1998) to characterize regional variability in the temporal and spatial distribution of species. This analysis is conducted to contrast how regional variations in the distribution of species compares with local variations in successional patterns predicted by log-linear analysis.

- In chapter 6 I describe the development of a nonlinear Markov chain of a rocky subtidal community. The model is parameterized using maximum likelihood methods to estimate density-dependent transition probabilities from the Gulf of Maine data set. I analyze the best fitting Markov chains to study the effects of species interactions on community dynamics, to characterize the sensitivity of community dynamics to initial conditions, and to identify multiple stable states in the subtidal system. I also examine how changes in the strength of interactions among species affect the behavior of the model.

- Finally, Chapter 7 summarizes the main results as they relate to the general theme of this thesis, and proposes some further research directions. 


\subsection{References}

Antolin, M.F. and J.F. Addicott. 1991. Colonization, among shoot movement, and local population neighborhoods of two aphid species. Oikos, 61:45-53.

Barradas, I., H. Caswell and J. E. Cohen. 1996. Competition during colonization vs. competition after colonization in disturbed environments: A metapopulation approach. Bulletin of Mathematical Biology, 58:1187-1207.

Bascompte, J. and R.V. Sole. 1996. Habitat fragmentation and extinction thresholds in spatially explicit models. Journal of Animal Ecology 65:465-473.

Bevers, M. and C.H. Flather. 1999. Numerically exploring habitat fragmentation effects on populations using cell-based coupled map lattices. Theoretical Population Biology, 55:61-76.

Caswell, H. and J. E. Cohen. 1991a. Communities in patchy environments: a model of disturbance, competition, and heterogeneity. In Ecological Heterogeneity, J. Kolasa (ed.). Springer-Verlag, Berlin.

Caswell, H. and J. E. Cohen. 1991b. Disturbance and diversity in metapopulations. Biological Journal of the Linnaean Society, 42:193-218.

Caswell, H. and R.J. Etter. 1993. Ecological interactions in a patchy environment: from patch occupancy models to cellular automata. In Patch Dynamics, S. A. Levin, T. M. Powell, and J. H. Steele (eds.), pp. 93-109. Springer Verlag, New York.

Caswell, H. and J. E. Cohen. 1995. Red, white and blue: environmental variance spectra and coexistence in metapopulations. Journal of Theoretical Biology, 176:301316. 
Caswell, H. and R.J. Etter. 1999. Cellular automaton models for competition in patchy environments: facilitation, inhibition, and tolerance. Journal Of Mathematical Biology, 61:625-649.

Cohen J.E., J.H.B. Kemperman, and Gh. Zbăganu. 1998. Comparisons of Stochastic Matrices; with applications in Information Theory, Statistics, Economics, and Population Sciences. Birkhäuser, Boston, MA.

Cohen, D. and S.A. Levin. 1991. Dispersal in patchy environments: the effects of temporal and spatial structure. Theoretical Population Biology, 39:63-99.

Connell, J.H. 1985. The consequences of variation in initial settlement vs. postsettlement mortality in rocky intertidal communities. Journal of Experimental Marine Biology and Ecology, 93:11-45.

Comins, H.N., M.P. Hassell and R.M. May. 1992. The spatial dynamics of hostparasitoid systems. Journal of Animal Ecology, 61:735-748.

Connolly, S. and J. Roughgarden 1998. A latitudinal gradient in northeast Pacific intertidal community structure: evidence for an oceanographically based synthesis of marine community theory. American Naturalist, 151:311-326.

Dayton, P.K., G.A. Robilliard and R.T. Paine. 1970. Benthic faunal zonation as a result of anchor ice at McMurdo Sound, Antarctica. In Antarctic ecology. Martin Holdgate ed., Academic Press, London.

Durrett, R. and S.A. Levin 1994. The importance of being discrete (and spatial). Theoretical Population Biology, 46:363-394.

Dytham, C. 1995. The effect of habitat destruction pattern on species persistence: a cellular model. Oikos, 74:340-344. 
Gaines, S. and J. Roughgarden. 1985. Larval settlement rate: a leading determinant of structure in an ecological community of the marine intertidal zone. Proceedings of the National Academy of Science, 82:3707-3711.

Grosberg and Levitan. 1992. For adults only? Supply-side ecology and the history of larval biology. Trends in Ecology and Evolution, 7:130-133.

Hassell, M.P., H.N. Comins and R.M. May. 1991. Spatial structure and chaos in insect populations. Nature, 353:255-258.

Hastings, A. 1980. Disturbance, coexistence, history, and competition for space. Theoretical Population Biology, 18:363-373.

Hastings, A. 1983. Can spatial variation alone lead to selection for dispersal?. Theoretical Population Biology, 24:244-251.

Horn, H.S. 1971. The adaptive geometry of trees. Princeton University Press, Princeton, New Jersey.

Horn, H.S. and R.H. MacArthur. 1972. Competition among fugitive species in a harlequin environment. Ecology, 53:749-752.

Horn, H.S. 1975. Markovian properties of forest succession. pp. 196-211 in M.L. Cody and J.M. Diamond (eds.) Ecology and evolution of communities. Harvard University Press, Cambridge, MA.

Hanski, I. 1994. Patch-occupancy dynamics in fragmented habitats. Trends in Evolution and Ecology, 4:131-135.

Hanski, I. 1999. Metapopulation ecology. Oxford University Press, Oxford.

Kareiva, P. 1987. Habitat fragmentation and the stability of predator-prey interactions. Nature, 321:388-391. 
Kareiva, P. 1990. Population dynamics in spatially complex environments: theory and data. In Population Regulation and Dynamics, (eds.) R.F. Denno and M.S. McClure), pp. 259-290. Academic Press, New York.

Kareiva, P. and V. Wennergren. 1995. Connecting landscape patterns to ecosystem and population processes. Nature, 373:299-302.

Karlin, S. and J. McGregor. 1972. Polymorphisms for genetic and ecological systems with weak coupling. Theoretical Population Biology, 3:210-238.

Kotliar, N.B. and J.A. Weins. 1990. Multiple scales of patchiness and patch structure: a hierarchical framework for the study of heterogeneity. Oikos, 59:253-260.

Lamberson, R.H., B.R. Noon, C. Voss and K.S. McKelvey. 1994. Reserve design for territorial species: The effects of patch size and spacing on the viability of the northern spotted owl. Conservation Biology, 8:185-195.

Lande, R. 1987. Extinction thresholds in demographic models of territorial populations. American Naturalist, 130:624-635.

Levin, S.A. 1974. Dispersion and population interactions. American Naturalist, 108:207-223.

Levin, S.A. 1992. The problem of pattern and scale in ecology. Ecology, 73:1943-1967.

Levin, S.A. and R.T. Paine. 1974. Disturbance, patch formation and community structure. Proceedings of the National Academy of Sciences, 71:2744:2747.

Levin S.A., D. Cohen and A. Hastings. 1984. Dispersal strategies in patchy environments. Theoretical Population Biology, 26:165-191.

Levins, R. 1969. Some demographic and genetic consequences of environmental heterogeneity for biological control. Bulletin of the Entomological Society of America, 15:237-240. 
Mackay, G. and N. Jan. 1994. Forest fires as critical phenomena. Journal of Physics A: Mathematical and General 17:L757-L760.

Menge B.A. and J.P. Sutherland. 1987. Community regulation: variation in disturbance, competition, and predation in relation to environmental stress and recruitment. American Naturalist, 130:730-757.

Molofski, J. 1994. Population dynamics and pattern fluctuations in theoretical populations. Ecology, 75:30-39.

Nee, S. and R.M. May. 1992. Dynamics of metapopulations: habitat destruction and competition coexistence. Journal of Animal Ecology, 61:37-40.

Noon, B.R. and K.S. McKelvey. 1996. A common framework for conservation planning: Linking individual and metapopulation models. In Metapopulations and Wildlife Conservation, D.R. McCullough (ed.), pp. 139-166. Island Press, Washington, D.C.

Paine, R.T. 1984. Ecological determinism in the competition for space. Ecology, 65:1339-1348.

Paine, R.T. and S.A. Levin 1981. Intertidal landscapes: disturbance and the dynamics of pattern. Ecological Monographs, 51:145-178.

Pickett S.T.A. and P.S. White 1985. Ecology of Natural Disturbance and Patch Dynamics. Academic Press, San Diego.

Roughgarden, J. 1977. Patchiness in the spatial distribution of a population caused by stochastic fluctuations in resources. Oikos, 29:52-59.

Sebens K.P. 1986. Spatial relationships among encrusting marine organisms in the New England subtidal zone. Ecol. Monogr., 56:73-96.

Shorrocks, B. 1991. Competition on a divided and ephemeral resource: a cage experiment. Biological Journal of the Linnean Society, 43:211-220. 
Silverton, J., S. Holtier, J. Johnson and P. Dale. 1992. Cellular automata models of interspecific competition for space - the effect of pattern on process. Ecology, 80:527-534.

Sole, R.V. and J. Valls 1991. Order and chaos in a 2D Lotka-Volterra coupled map lattice. Physics Letters A, 153:330-336.

Steel, J.H. 1978. Spatial Patterns in Plankton Communities. Plenum, New York.

Stephens, D.W. and J.R. Krebs. 1986. Foraging Theory. Princeton University Press, Princeton, NJ.

Sutherland, B.R. and A.E. Jacobs. 1994. Self-organization and scaling in a lattice predator-prey model. Complex Systems, 8:385:405.

Taylor, L.R. 1961. Aggregation, variance and the mean. Nature, 189:732-735.

Taylor R.A.J. and L.R. Taylor. 1979 A behavioral model for the evolution of spatial dynamics. In Population dynamics. (eds.) R.M. Anderson, B.D. Turner and L.R. Taylor, pp 1-28. Blackwell, Oxford.

Tanner J.E., T.P. Hughes and J.H. Connell. 1994. Species coexistence, keystone species, and succession: a sensitivity analysis. Ecology, 75:2204-2219.

Tanner J.E., T.P. Hughes and J.H. Connell. 1996. The role of history in community dynamics: a modeling approach. Ecology, 77:108-117.

Tilman, D. and P. Kareiva. 1997. Spatial ecology. Princeton University Press, Princeton, New Jersey.

Usher, M.B. 1979. Markovian approaches to ecological succession. Journal of Animal Ecology 48:413-426.

Wiens, J.A. 1997. Metapopulation dynamics and landscape ecology. In Metapopulation biology. (eds.) I.A. Hanski and M.E. Gilpin, pp 43-68. Academic Press, San Diego. 
Weins, J.A., N.C. Stenseth, B. Van Horne and R.A. Ims. 1993. Ecological mechanisms and landscape ecology. Oikos, 66:369-380.

Witman J.D. 1987. Subtidal coexistence: storms, grazing mutualism, and the zonation of kelp and mussels. Ecol. Monogr., 57:167-187.

Witman, J.D. and P.K. Dayton. 2000. Rocky subtidal communities. In Bertness, M.D. and M. Hay eds. Marine Benthic Ecology. Sinauer Press (in press)

Wu, J. and S.A. Levin 1994. A spatial patch dynamic modeling approach to pattern and process in an annual grassland. Ecological Monogrpahs,64:447-464.

Wu, J. and O.L. Loucks. 1995. From balance of nature to hierarchical patch dynamics: a paradigm shift in ecology. The Quarterly Review in Biology, 70:439-466. 


\section{Chapter 2}

\section{Habitat fragmentation and extinction thresholds on fractal landscapes}

The earth belongs to the living, not to the dead.

-Thomas Jefferson

\section{$2.1 \quad$ Introduction}

A species living in a fragmented landscape, only part of which is suitable for occupancy, faces two challenges for persistence. It must balance mortality and reproduction within a patch to maintain itself locally, and must locate those parts of the landscape that are suitable. As landscapes become more fragmented due to human disturbance, the second challenge becomes a critical conservation and management issue.

Habitat fragmentation is important because there exists a threshold level of suitable habitat, below which the population goes extinct, even though its vital rates are capable of supporting positive population growth in the suitable areas. This was first shown by Lande (1987) in an analysis of the Northern Spotted Owl, which is

\footnotetext{
${ }^{1}$ This chapter was published in Ecology Letters (1999) 2:121-127.
} 
endangered because logging has fragmented its old-growth habitat. This result has become a paradigm in conservation biology (e.g. McKelvey et al. 1993; Lamberson et al. 1992).

Lande's model is an ingenious application of demographic theory, but does not directly describe the dynamics of occupied and unoccupied territories. He incorporated the fraction of suitable territories as a factor in a survivorship function, and calculated the net reproductive rate by substituting this survivorship function into Lotka's integral equation of population growth. By setting the net reproductive rate to 1 , he solved for the minimum fraction of suitable habitat permitting population growth.

An alternative approach is to use metapopulation (Levins 1969) or patch-occupancy (Caswell and Cohen 1991a, b, 1995) models to investigate the affects of habitat fragmentation. Such models describe the landscape as a mosaic of discrete patches and focus on the balance between colonization and local extinction. Several single-species metapopulation models have shown that a population cannot persist when the fraction of suitable patches or territories in the landscape falls below a critical threshold (Nee and May 1992; Lamerson and Carroll 1993; Kareiva and Wennergren 1995; Noon and McKelvey 1996). Nee and May (1992) extended this approach to a two species competition model and found that habitat destruction decreased the frequency of patches occupied by the superior competitor, but surprisingly increased the frequency of the inferior competitor. Multi-species competition models (Tillman et al. 1994; Stone 1995) have shown that the most vulnerable species to habitat loss are the top competitors (given that they have lower colonization rates), and that species extinction may occur decades after suitable territory has been destroyed (the so called extinction debt; Tillman et al. 1994).

Because these models assume that every patch interacts equally with every other patch, the explicit arrangement of patches has no effect on the results, and such models tell us nothing about how the spatial arrangement of habitat destruction 
effects a population. These effects can be important (Doak et al. 1992; Mckelvey et al. 1993; Bascompte and Sole 1996).

In this paper, we derive a discrete time patch-occupancy model (PO model) which captures the essential features of Lande's model and the other single-species models in the literature. We then embed this model in an explicit spatial arrangement of habitat destruction by transforming it into a stochastic cellular automata model (Caswell and Etter 1992, 1999). We compare the results of the two models to study the effects of the spatial arrangement of habitat destruction.

\subsubsection{Lande's Model}

Suppose that the landscape is divided into patches the size of an individual territory, and that a proportion $h$ of these are suitable and a proportion $u$ are unsuitable. Let $p$ denote the proportion of the suitable territories that are occupied. Suppose that individuals with a suitable territory have a survivorship function $l^{\prime}(x)$ and maternity function $b(x)$. At some age before maturity, juveniles inhabit their parents territory with probability $\varepsilon$ or disperse in search of suitable territory. A juvenile can search as many as $m$ potential territories before dying. The probability of finding an unoccupied and suitable territory is

$$
1-(1-\varepsilon)(u+p h)^{m}
$$

Since dispersal happens before maturity, the survivorship function for ages beyond maturity is simply $l^{\prime}(x)$ multiplied by (2.1). Thus the net reproductive rate is

$$
R_{0}=1-(1-\varepsilon)(u+p h)^{m} \int_{a}^{\infty} l^{\prime}(x) b(x) d x
$$

where $a$ is the age of maturity. Denoting the integral term by $R_{0}^{\prime}$ (i.e. the net reproduction rate conditional on finding a territory), the condition for equilibrium is

$$
1-(1-\varepsilon)(u+p h)^{m} R_{0}^{\prime}=1
$$


The only variable in this equation is $p$; the value which satisfies it is the equilibrium habitat occupancy

$$
p^{*}=\left\{\begin{array}{r}
1-\frac{1-k}{h} \\
0
\end{array}\right.
$$

Where

$$
\dot{k}=\left(\frac{1-\frac{1}{R_{0}^{\prime}}}{1-\varepsilon}\right)^{1 / m}
$$

Since $p^{*}=k$ when $h=1$, Lande identifies $k$ as the demographic potential of the population.

Plotting $p^{*}$ as a function of $h$ for different values of $k$ reveals a clear extinction threshold; if $h$ is too small the population goes extinct.

\subsubsection{Metapopulation Models}

Levins (1969) envisioned metapopulation dynamics as a tradeoff between the colonization rate $C$ of empty patches and the local extinction rate $m$ of occupied patches. If we let $x$ denote the fraction of empty patches, then the instantaneous change in the proportion of occupied patches is

$$
\frac{d x}{d t}=C x(1-x)-m x
$$

The non-trivial steady state solution is $x^{*}=1-\frac{m}{C}$, so the population persists only if $C>m$. Note that this solution implies that for biologically realistic values of $C$ and $m$ the metapopulation is unable to occupied all available patches.

Habitat destruction can be incorporated into (2.6) by introducing a term $D$ denoting the proportion of patches that have been destroyed. In a landscape where not all of the habitat is suitable, the change in the frequency of occupied patches becomes

$$
\frac{d x}{d t}=C x(1-D-x)-m x
$$


where the non-trivial solution is now $x^{*}=1-D-\frac{m}{C}$. Thus for the population to persist, $D$ must be less than the critical destruction level $D_{c}=1-\frac{m}{C}$. Equation (2.7) can be solved for the proportion of suitable territories occupied by making the substitutions $h=1-D$ and $p=\frac{x}{h}$, yielding

$$
\frac{d p}{d t}=C h p(1-p)-m p
$$

At equilibrium $p^{*}=1-\frac{m}{h C}$, and the population goes extinct when $h \leq \frac{m}{C}$.

\subsection{The Patch-Occupancy Model}

Lande's model takes a population-centered view of the landscape, and calculates the fraction of occupied territory from consideration of the survival and reproduction of individuals. Metapopulation models take a patch-centered view where occupied and unoccupied patches change states in continuous time, depending on colonization and mortality rates. A patch-occupancy model also takes a patch-centered view, however, patches are now described as state variables which change states according to a discrete time nonlinear Markov process.

Consider a landscape composed of patches, each of which can be in one of three states

\begin{tabular}{r|l} 
State & Description \\
\hline 1 & Suitable habitat; unoccupied \\
2 & Suitable habitat; occupied \\
3 & Unsuitable habitat
\end{tabular}

Denote the number of patches in state $i$ by $n_{i}$, and the proportion of patches in state $i$ by $x_{i}$, for $i=1,2,3$. In the model, the proportion $h$ of suitable habitat is constant; $h=x_{1}+x_{2}$. We denote the proportion of suitable patches that are actually occupied by $p=x_{2} / h$. This is the variable of interest in the conservation context. 
No one expects to find owls in parking lots; what is important is how much of their remaining potential habitat is actually occupied.

We will speak of the colonization, growth, and local extinction of populations within patches, but the model can also be interpreted in terms of the immigration and mortality of individuals on territories.

A population in an occupied territory goes extinct in a unit of time with probability $\delta$, and survives with probability $1-\delta$ (Figure 2.1). Local extinction produces an empty patch. The colonization of such a patch is described by a Poisson process. Colonists arrive at the empty patch from other occupied patches in the system. Thus the probability that an unoccupied territory is colonized is

$$
\begin{aligned}
C & =P[\text { at least } 1 \text { colonist arrives }] \\
& =1-e^{-M}
\end{aligned}
$$

where $M$ is the mean number of colonist arriving at a territory. That number is given as

$$
\begin{aligned}
M & =\frac{d n_{2}}{n_{1}+n_{2}+n_{3}} \\
& =b x_{2}
\end{aligned}
$$

where $b$ is the number of dispersing propagules produced by each of the $n_{2}$ occupied patches, and $n_{1}+n_{2}+n_{3}$ is the number of patches over which those propagules are distributed. Thus $b$ can be interpreted as a measure of propagule production; we will call it the dispersal parameter. In terms of $b$, the colonization probability can be written as

$$
C=1-e^{-b x_{2}}
$$




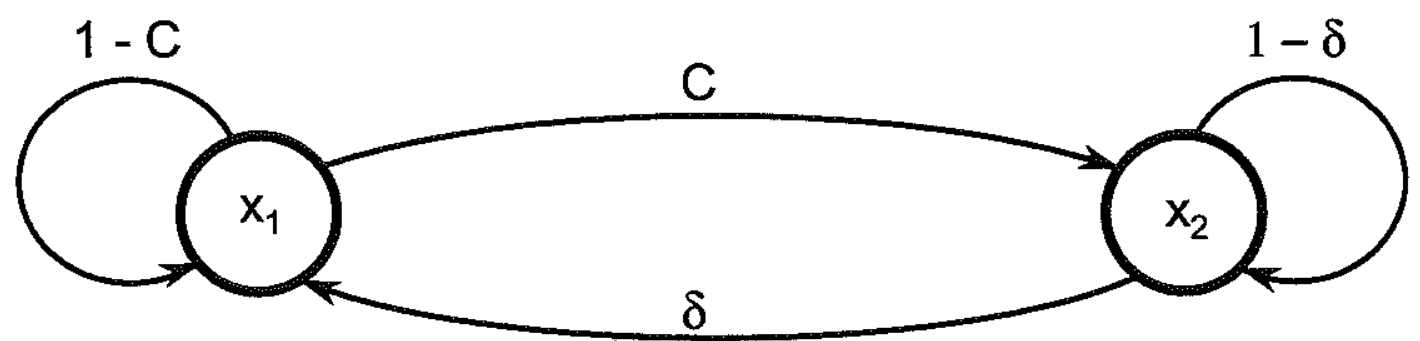

Suitable habitat; unoccupied

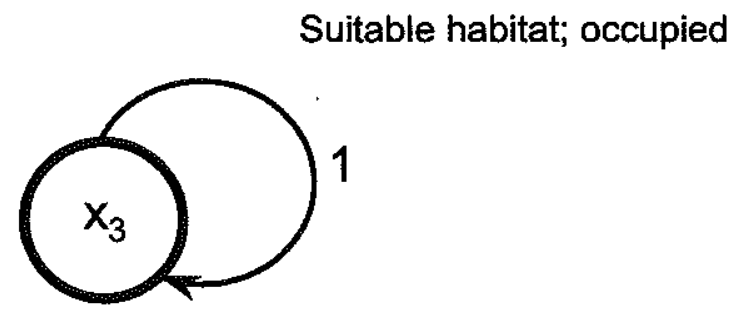

Unsuitable habitat

Figure 2.1: Transition diagram for the patch-occupancy model. The circles represent the different possible states of a patch, and the arrows show the possible state transitions. $C$ is the probability that a suitable unoccupied patch is colonized and $\delta$ is the probability that occupied patch goes extinct. See text for details. 
The dynamics of the system are given by

$$
\begin{aligned}
& x_{1}(t+1)=(1-C) x_{1}(t)+\delta x_{2}(t) \\
& x_{2}(t+1)=C x_{1}(t)+(1-\delta) x_{2}(t) \\
& x_{3}(t+1)=x_{3}(t)
\end{aligned}
$$

Because the proportion of suitable patches, $h$, is constant and equals $x_{1}+x_{2},(2.12)$ can be reduced to a single equation describing the dynamics of occupied patches

$$
x_{2}(t+1)=C\left(h-x_{2}(t)\right)+(1-\delta) x_{2}(t)
$$

where $C=1-e^{-b x_{2}(t)}$.

Equation (2.13) can be rewritten in terms of the occupancy $p$ of suitable patches as

$$
p(t+1)=\left(1-e^{-h b p(t)}\right)(1-p(t))+(1-\delta) p(t) .
$$

As $h$ decreases the colonization rate decreases and fewer empty patches are colonized during each time step.

\subsubsection{Stability Analysis and Extinction Threshold}

Equation (2.14) has two fixed points: a trivial equilibrium at $p=0$ and another satisfying

$$
\hat{p}=\frac{1-e^{-h b \hat{p}}}{1-e^{-h b \hat{p}}+\delta}
$$

The species can invade the system when the trivial equilibrium is unstable. Denote the right hand side of equation $(2.14)$ by $f(p, h)$. The boundary between stability and instability of the trivial equilibrium is given by

$$
\left.\frac{\partial f(p, h)}{\partial p}\right|_{p=0}=1 .
$$


The value of $h$ satisfying this equation is $h_{c}$, the critical proportion of suitable habitat. It satisfies

$$
\left(1+h_{c} b\right)-\delta=1
$$

the solution to which is

$$
h_{c}=\frac{\delta}{b}
$$

Populations with a higher dispersal rate or a smaller extinction rate can tolerate more habitat destruction.

A transcritical bifurcation occurs at $h=h_{c}$, at which the trivial equilibrium ( $p=$ 0 ) and the nontrivial equilibrium $(\hat{p})$ collide and exchange stability. The conditions for such a bifurcation are (Wiggins 1990)

$$
\begin{aligned}
\frac{\partial f}{\partial h} & =b p e^{-h b p}(1-p)=0 \\
\frac{\partial^{2} f}{\partial p \partial h} & =e^{-h b p}[b(1+p(h b p-h b-2))] \neq 0 \\
\frac{\partial^{2} f}{\partial p^{2}} & =h b e^{-h b p}[h b(p-1)-2] \neq 0
\end{aligned}
$$

where all the derivatives are evaluated at $p=0$ and $h=h_{c}$. It is easy to confirm upon substitution that all three conditions are met.

The nontrivial equilibrium $\hat{p}$ is always stable when it exists. To see this, note that the second derivative (2.21) is negative for $0 \leq p \leq 1$, so the one-dimensional map of equation (2.14) is concave downward (Fig. 2.2). Since the map is always concave downward, $\hat{p}$ can lose stability only by a flip bifurcation, i.e. when $\partial f / \partial p<-1$. However, this would require that

$$
e^{-h b \hat{p}}<\frac{\delta-1}{1+h b(1-\hat{p})}
$$

But the left side of (2.22) is always positive and the right side is always negative. Hence, the inequality never holds, and flip bifurcations are impossible. 


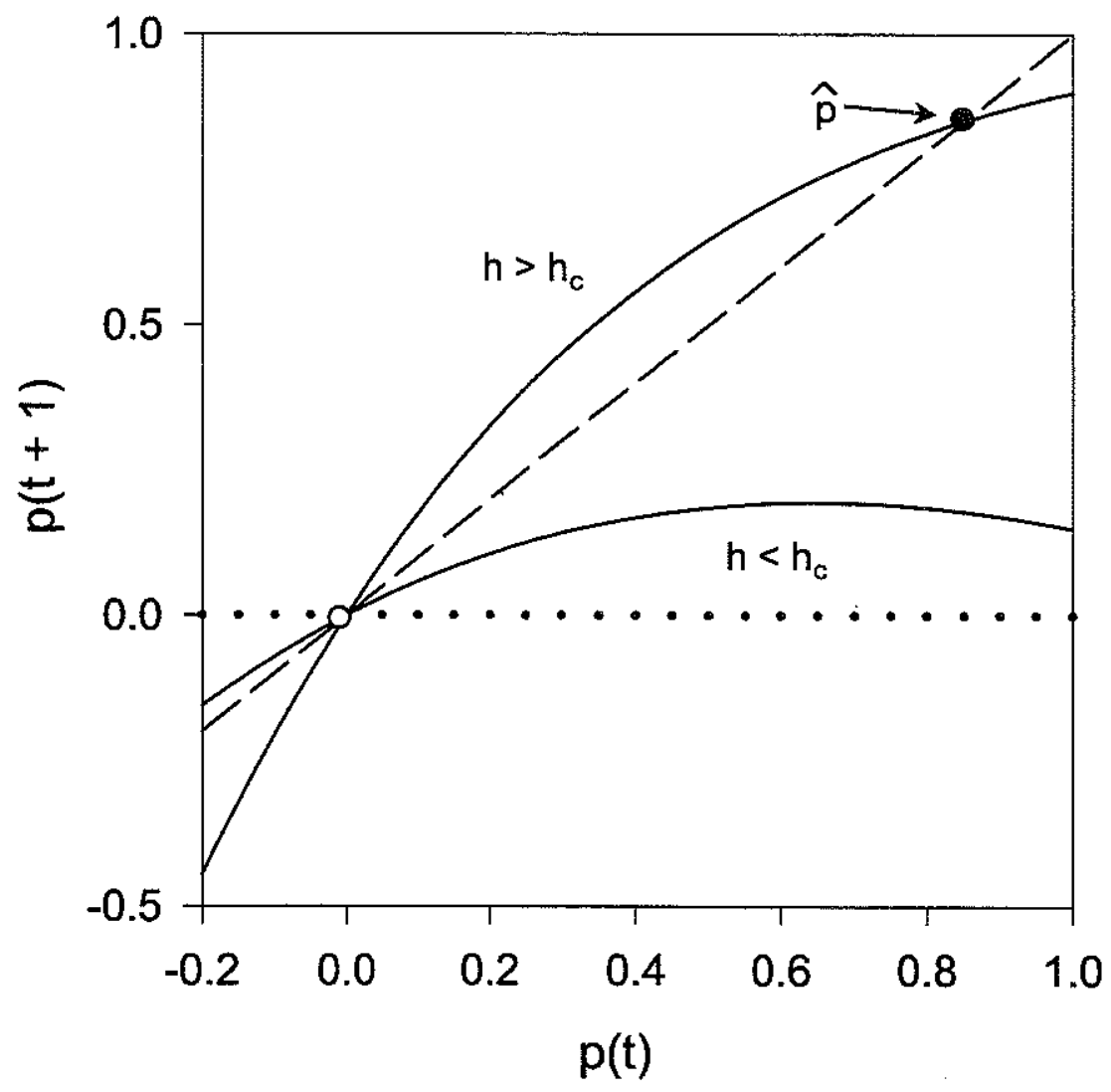

Figure 2.2: A one-dimensional map for equation (2.14), where the solid line in each plot depicts $p(t+1)$ as a function of $p(t)$. The top curve shows the case for $h>h_{c}$ while the bottom curve is for $h<h_{c}$. Fixed points are located at the intersection of the curves with the $45^{\circ}$ dashed line (point at which $p(t+1)=p(t)$ ). 

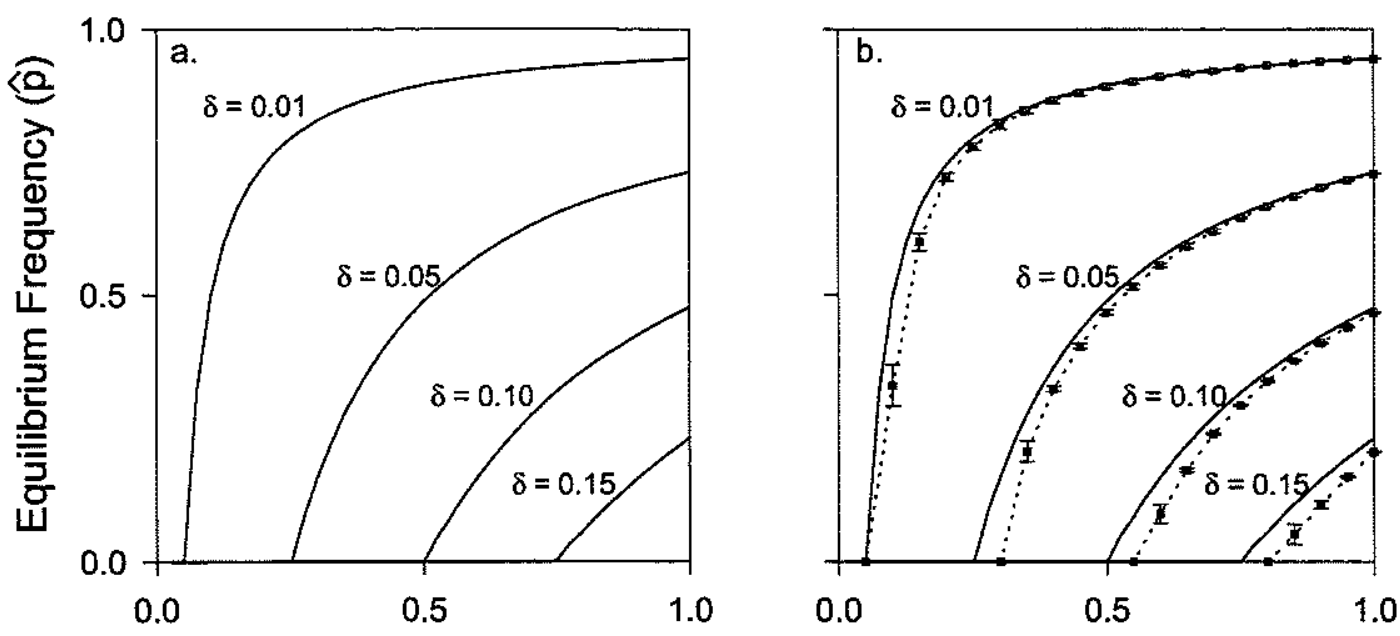

Suitable habitat (h)

Figure 2.3: Equilibrium frequency of occupied patches, $\hat{p}$, as a function of $h$ for $(a$.) the PO model, and $(b$. ) the CA (dashed line) when patches are randomly destroyed. CA results are averages from five replicate simulations. The error bars represent $95 \%$ confidence intervals. The curves are for $b=0.2$ and $\delta=0.01,0.05,0.10$, and 0.15 .

Thus, the long-term dynamics of the model are limited to either extinction (when $h<h_{c}$ ), or convergence to a stable equilibrium (when $h>h_{c}$ ). The equilibrium frequency, $\hat{p}$, of occupied patches can be found by solving (2.15) numerically. Figure 2.3a shows $\hat{p}$ as a function of $h$; the pattern agrees with the results of Lande's model for the Northern Spotted Owl (Lande 1988).

\subsection{Adding Landscape Structure}

To study the influence of landscape structure on dynamics we converted the patch occupancy model into a cellular automaton (CA) (see Caswell and Etter 1993, 1999, Etter and Caswell 1994, for the relationship between patch-occupancy and CA models of this sort). In the $\mathrm{CA}$, both the state and the location of patches are followed explicitly through time. Each patch evolves in time following (2.12), but interacts only with patches in a local neighborhood. 
We simulated the CA on a $256 \times 256$ grid with periodic boundary conditions. Each patch is in one of three states; unoccupied but suitable for colonization, occupied, or unsuitable. Patches change state according to a nonlinear Markov chain whose transition matrix is

$$
\mathbf{A}=\left(\begin{array}{ccc}
1-C & \delta & 0 \\
C & 1-\delta & 0 \\
0 & 0 & 1
\end{array}\right)
$$

The elements of $\mathbf{A}$ correspond to the transition probabilities in Figure 2.1. The colonization probability $C$ is given by

$$
C=1-\exp \left(-b x_{2}^{(N)}\right)
$$

where $x_{2}^{(N)}$ is the frequency of occupied patches in the neighborhood of the patch under consideration. We report results here for a $7 \times 7$ neighborhood, which is equivalent to a dispersal radius of 3 patches in all directions from an occupied patch. The parameters in the CA model are the same as those in the PO model: $\delta$ is the extinction probability of a local population within a patch, and $d$ is the dispersal parameter.

\subsubsection{Fractal Landscapes}

In a spatially explicit model the pattern of habitat fragmentation may also affect population dynamics. The simplest pattern is a random uncorrelated distribution of suitable and unsuitable patches. Real landscapes, however, are often fractal, showing patterns of variance or clumping at different spatial scales (Mandelbrot 1982; Krummel et. al. 1987; Milne 1988; Sole and Manrubia 1995).

We created landscapes with fractal patterns of habitat destruction from fractal surfaces whose contours are the traces of fractal Brownian motion. If $V(t)$ represents the value of a randomly moving trace at time $t$, then the change, $\Delta V=V\left(t_{2}\right)-V\left(t_{1}\right)$, 
over the increment $\Delta t=t_{2}-t_{1}$, scales as

$$
\Delta V \propto \Delta t^{H}
$$

where the ruggedness of the trace is controlled by the hurst exponent $H$ (Saupe 1988). In 3-dimensional space the path of the trajectory produces a fractal surface. These surfaces are now familiar from computer graphics books and movies and appear remarkably similar to real landscape surfaces (Peitgen and Saupe 1988).

The intersection of the surface with a horizontal plane ("flooding" the landscape to a specific elevation) creates two sets of patches: those above the plane and those below it. By defining the patches below the plane as unsuitable and then progressively flooding the landscape to higher elevations, we create fragmented habitats in which the frequency of suitable patches declines from 1 to 0 , and in which the suitable patches are self-similar throughout.

The edges of these patches have a fractal dimension $\mathrm{D}$ given by.

$$
D=2-H
$$

where $1 \leq D \leq 2$. As $D$ increases, fragment edges become increasingly rough, and suitable patches tend to be isolated in small clusters. Their connectivity is minimized when $D=2$. On the other hand, as $D \rightarrow 1$, fragment edges become smoother, and suitable patches are more likely to be located within large contiguous fragments than in small isolated clusters.

We used the midpoint displacement algorithm published in Saupe (1988) to produce random surfaces with $D=1.9,1.5$, and 1.1. We generated five replicate random landscapes for each fractal dimension. Examples are shown in Figure 2.4 with $50 \%$ suitable habitat. An uncorrelated random landscape, created by allocating suitable and unsuitable patches randomly and independently, is also shown for comparison (Fig. 2.4d). 
a.
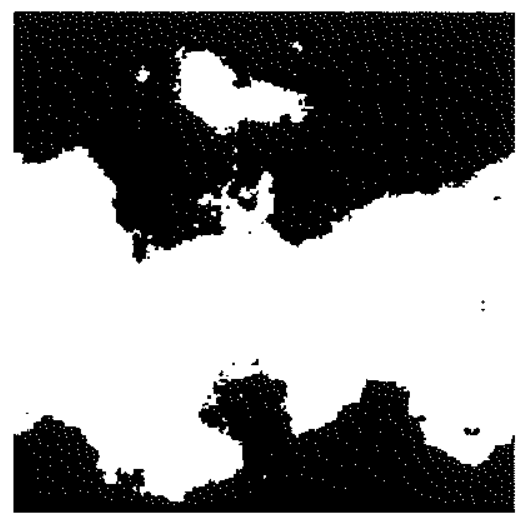

c.

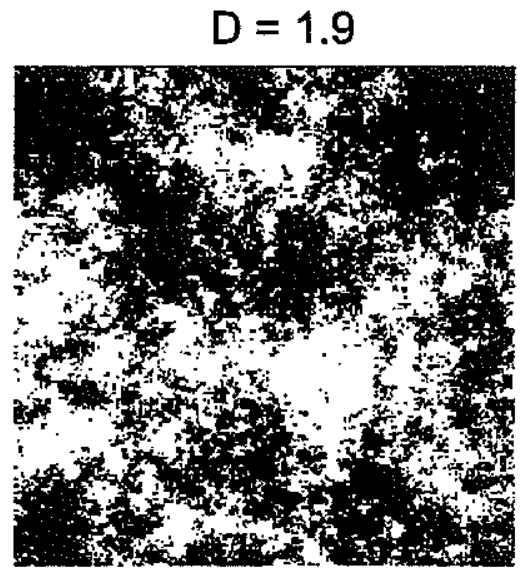

b.

$D=1.5$

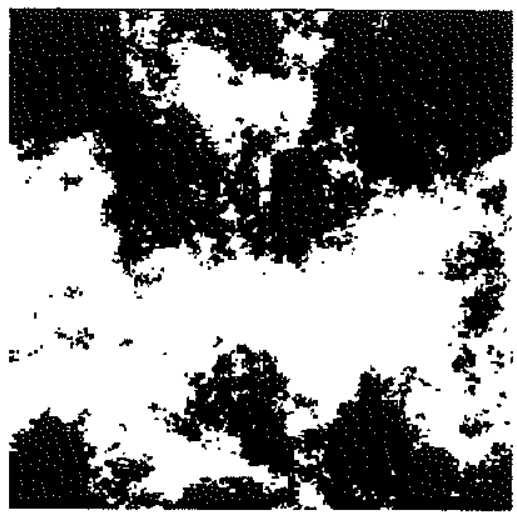

d.

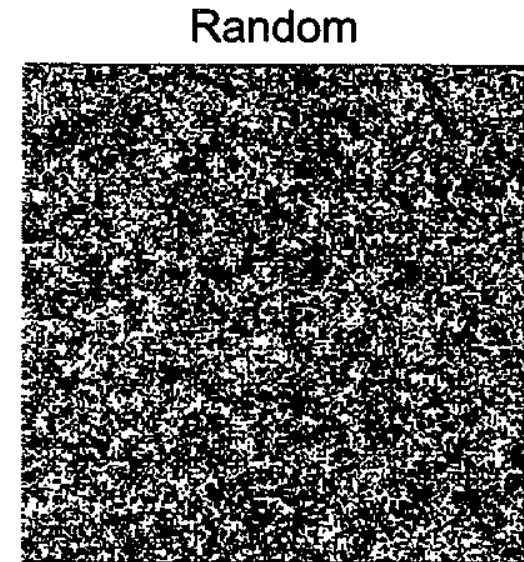

Figure 2.4: Three Browian fractal landscapes (a-c) produced using the midpoint displacement algorithm (Barnsley et al.). Landscapes are depicted with $50 \%$ suitable habitat. White corresponds to regions consisting of suitable patches. Black areas represent destroyed patches. A landscape in which $50 \%$ of the patches were randomly destroyed (d) is included for comparison. The fractal landscapes were produced using the same integer value to seed the random number generator. 


\subsection{Simulation Methods}

Before the start of each simulation, a proportion of patches in the landscape were designated as unsuitable. The location of unsuitable patches were assigned either randomly and independently (Fig. 2.4d), or by superimposing the CA onto one of the fractal surfaces (Fig. 2.4a-c). The CA was initialized by setting all suitable patches to state 2 (occupied), and run until the proportion of occupied patches converged to a stable equilibrium. A simulation was considered to have reached equilibrium when the absolute value of the slope of a regression line fit to the last 100 values of $p(t)$ was less than 0.01 .

A value of $b=0.2$ was used for all CA simulations (different values of $d$ give qualitatively similar results for the same value of $\delta / d$ ). Local extinction probabilities were varied between $\delta=0.01$ and $\delta=0.19$ so that $0<\delta / b<1$. Five replicate simulations were performed for each value of $h$ and for each set of parameter values.

\subsection{Results}

Figure 2.3b shows equilibrium frequencies $(\hat{p})$ as a function of $h$ for different values of $\delta$ on a random uncorrelated landscape. The behavior of the spatial model on such a landscape is similar to that of the non-spatial model. As the proportion of suitable habitat decreases, $\hat{p}$ decreases until a threshold is reached at which the population goes extinct. The equilibrium frequency $\hat{p}$ decreases. faster in the $\mathrm{CA}$ than in the PO model, and the critical proportion of suitable habitat required for persistence is larger, especially for higher disturbance probabilities $(\delta \geq 0.05)$, but the spatially explicit model creates no qualitatively new results.

When habitat is destroyed in a fractal pattern, the behavior of the CA changes dramatically (Fig. 2.5). The equilibrium frequency $(\hat{p})$ on a fractal landscape is much higher than on a random landscape or in the PO model. For a given amount of suitable habitat, $\hat{p}$ varies inversely with the fractal dimension of the landscape, i.e. 


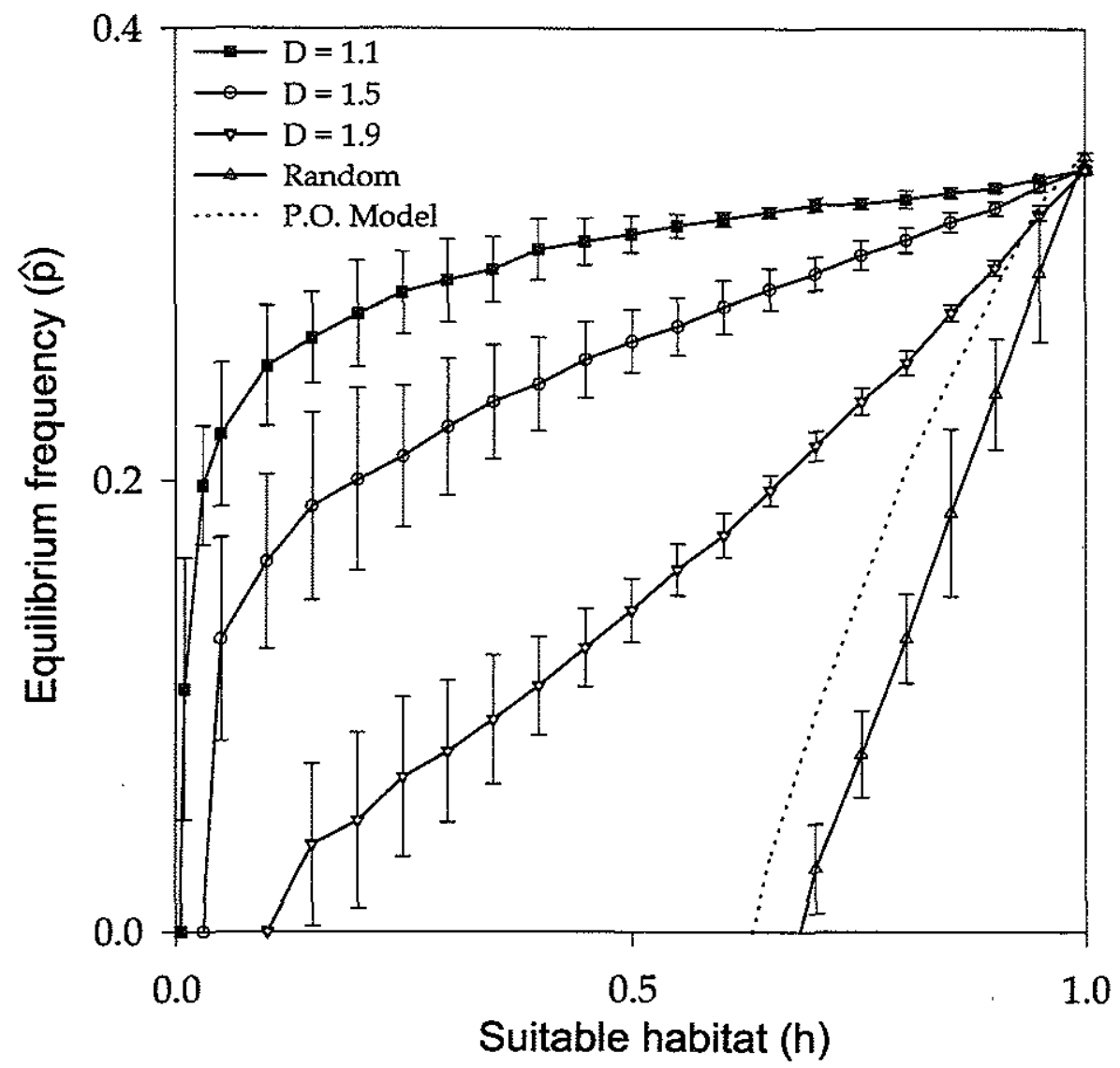

Figure 2.5: Equilibrium frequency of occupied patches, $\hat{p}$, as a function of $h$, for the $\mathrm{CA}$ on landscapes with different fractal dimensions. Equilibrium curves for the PO model (dashed line), and the CA with random habitat destruction, are included for comparison. CA results are averages from five replicate simulations. The error bars represent $95 \%$ confidence intervals. Parameter values are $\delta=0.125$, and $b=0.2$. Statistically, values of $\hat{p}$ for $D=1.9,1.5$, and 1.1 are significantly different for $h<$ 0.95 . This pattern is qualitatively similar for all values of $\delta / b<0.9$.

the population does much better when suitable patches are more contiguous. This pattern is qualitatively the same for all values of $\delta / b<0.90$.

Figure 2.6 shows the extinction thresholds for the different habitat destruction regimes as a function of $\delta / b$. On a fractal landscape, the CA population can persist with much less suitable habitat than predicted by the PO model (except when $\delta / b>$ 0.925 , in which case the population always goes extinct, even if $h=1$ ). Decreasing the 


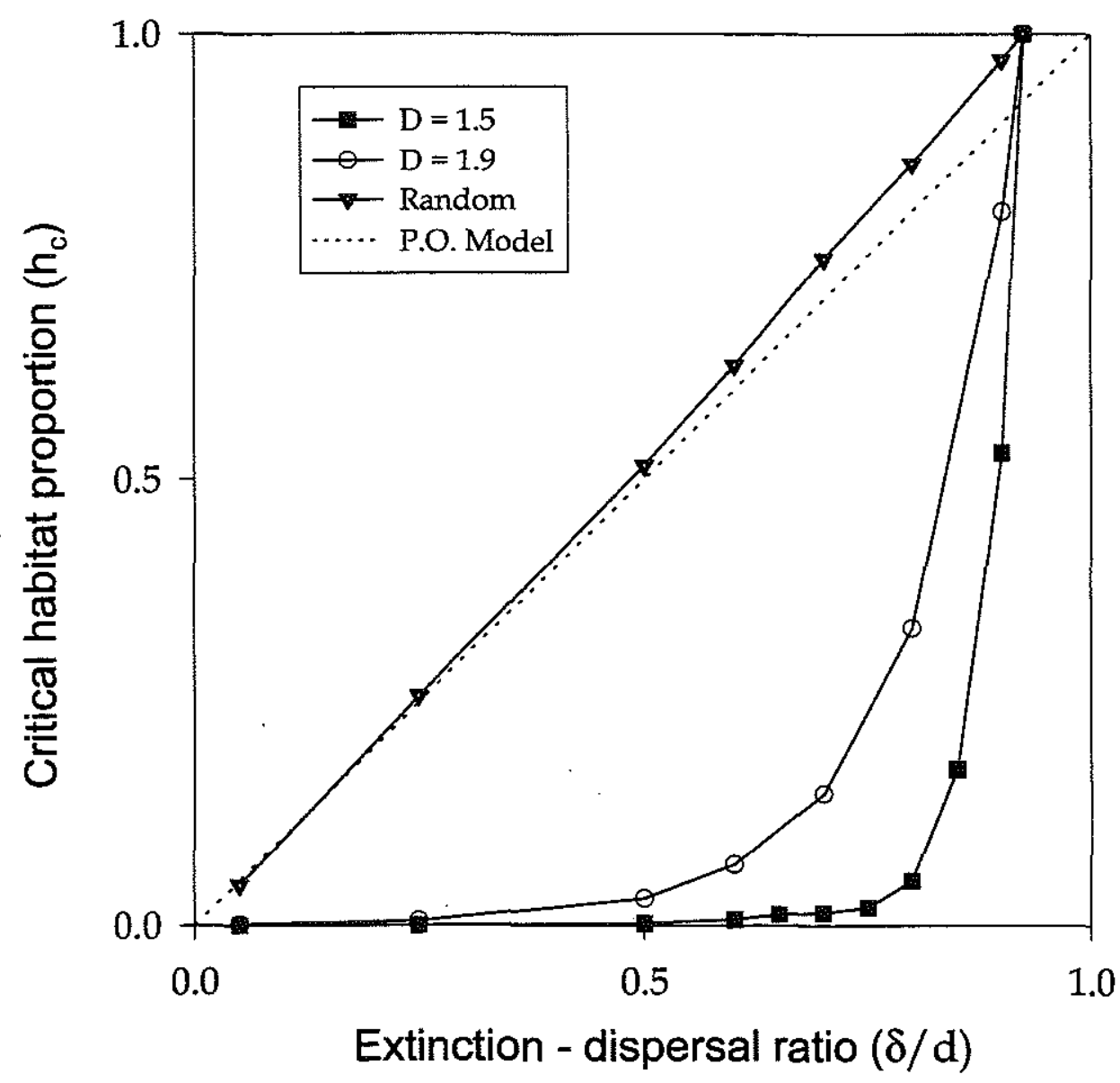

Figure 2.6: Extinction threshold, $h_{c}$, as a function of $\delta / b$ (for $b$ fixed at 0.2 ) on random and fractal landscapes. For the CA, $h_{c}$ is defined as the value of $h$ at which $\hat{p} \approx 0.01$. Each point represents the average estimated value of $h_{c}$ in five replicate landscapes. The dashed line gives the extinction threshold for the PO model.

fractal dimension of the landscape further decreases the amount of suitable habitat required for the population to persist. This is especially true when local extinction rates are higher $(0.5 \leq \delta / b \leq 0.9)$. 


\subsection{Discussion}

Spatially implicit models, such as Lande's demographic model and single species patch-occupancy models, have provided conservation biologist with valuable insights into the problem of habitat fragmentation. Patch-occupancy models, however, are mean field approximations to spatially explicit CA models (Caswell and Etter 1993, 1999). Comparisons between the two kinds of models provide insights into how the spatial arrangement of patches in a fragmented landscape affect population dynamics.

The amount of habitat loss that a population can tolerate depends on the spatial arrangement of suitable and unsuitable habitat. On an uncorrelated random landscape, the population occupies slightly less territory than predicted by the PO model, and is more susceptible to global extinction. The magnitude of this difference increases with the local extinction rate. This result is similar to work published by Bascompte and Sole (1996), who found that a population was more vulnerable to the effects of random habitat destruction in a spatially explicit metapopulation model than in its spatially implicit counterpart. Such findings would suggest that non-spatial models underestimate the effects of habitat fragmentation on population persistence.

In the real world, however, habitat destruction is rarely a completely random process. Instead, fragmentation tends to produce suitable territories of varying size with irregular boundaries that are often fractal in nature (Krummel et. al. 1987; Milne 1988; Scheuring 1991; Sole and Manrubia 1995). On such a fractal landscape, a population can tolerate greater habitat destruction, and has a lower extinction threshold, than predicted by the PO model. Persistence becomes even more favored as the fractal dimension of the landscape decreases. As $D \rightarrow 1$, suitable patches become more clumped together and the boundaries between suitable and unsuitable territory become smoother. This arrangement insures that on a local scale, patch dynamics remain relatively unaffected by habitat destruction outside the periphery of a cluster of suitable patches, since few propagules produced in these clusters end up 
in unsuitable territory. Thus a fractal arrangement of suitable patches increases the ability of a population to tolerate habitat destruction by facilitating the recolonization of empty suitable territory. This conclusion supports previous studies on reserve design for territorial species, which show that the likelihood of population persistence increases when suitable territories are clustered together (Doak 1989; Carroll and Lamberson 1993; Lamberson et al. 1994).

Our findings are consistent with those of Dythan (1995), who compared different habitat loss regimes in a cellular automata counterpart of a spatially implicit competition model proposed by Nee and May (1992). In their model, Nee and May showed that increased habitat destruction progressively decreases the absolute abundance of the superior competitor while increasing the relative abundance of the inferior competitor. In CA simulations, Dythan found that when destruction of patches was non-random, the relationship between the superior and inferior competitors changed in a similar way, but both species were able to persist in less habitat than predicted by Nee and May's model.

Although we have shown that a population on a fractal landscape can persist with a relatively small amount of suitable territory, the results presented here should not be taken as justification to continue destroying native habitats. Instead they are meant to point out the need to think about spatial structure and spatial scale when considering conservation strategies. It is unlikely that all the effects of landscape structure have been incorporated into our model. For instance, habitat connectivity can have adverse effects on populations by facilitating the spread of contagious diseases and increasing predation pressure. Models of this effect suggest that increasing territorial connectivity beyond a critical point can drive a population to extinction (Hess 1996). The interaction between such mortality rates and landscape structure in spatially explicit models will have to be reserved for future studies. We believe it is safe to say, however, that landscape structure plays a vital role in mediating the effects of habitat fragmentation on persistence. 


\section{$2.7 \quad$ References}

Bascompte, J. and R.V. Sole. 1996. Habitat fragmentation and extinction thresholds in spatially explicit models. Journal of Animal Ecology 65:465-473.

Carroll, J.E. and R.H. Lamberson. 1993. A continuous model for the dispersal of territorial species, SIAM. Journal of Applied Mathematics, 53:205-218.

Caswell, H. and J. E. Cohen. 1991a. Communities in patchy environments: a model of disturbance, competition, and heterogeneity. In Ecological Heterogeneity, J. Kolasa (ed.). Springer-Verlag, Berlin.

Caswell, H. and J. E. Cohen. 1991b. Disturbance and diversity in metapopulations. Biological Journal of the Linnaean Society, 42:193-218.

Caswell, H. and R.J. Etter. 1993. Ecological interactions in a patchy environment: from patch occupancy models to cellular automata. In Patch Dynamics, S. A. Levin, T. M. Powell, and J. H. Steele (eds.), pp. 93-109. Springer Verlag, New York.

Caswell, H. and J. E. Cohen. 1995. Red, white and blue: environmental variance spectra and coexistence in metapopulations. Journal of Theoretical Biology, 176:301316.

Caswell, H. and R.J. Etter. 1999. Cellular automaton models for competition in patchy environments: facilitation, inhibition, and tolerance. Journal Of Mathematical Biology, (Submitted).

Doak, D. 1989. Spotted owls and old growth logging in the Pacific Northwest. Conservation Biology, 3:389-396.

Doak, D. F., P.C. Marino and P.M. Kareiva. 1992. Spatial scale mediates the influence of habitat fragmentation on dispersal success: Implications for conservation. Theoretical Population Biology, 41:315-336. 
Durrett, R. and S. Levin. 1994. The importance of being discrete (and spatial). Theoretical Population Biology, 46:363-394.

Dytham, C. 1995. The effect of habitat destruction pattern on species persistence: a cellular model. Oikos, 74:340-344.

Etter, R.J. and H. Caswell. 1994. The advantages of dispersal in a patchy environment: Effects of disturbance in a cellular automata model. In Reproduction, Larval Biology and recruitment in the Deep-sea Benthos, Eckelbargar K. and Young C. (eds.), pp. 284-305. Columbia University Press, New York.

Groom, M.J. and N. Schumaker. 1993. Evaluating landscape change: Patterns of worldwide deforestation and local fragmentation. In Biotic Interactions and Global Change, Kareiva P.M., Kingsolver J.L., and Huey R.B. (eds.), pp. 24-44. Sinauer Associates Inc., Massachusetts.

Hanski, I. 1994. Patch-occupancy dynamics in fragmented habitats. Trends in Evolution and Ecology, 4:131-135.

Kareiva, P. and V. Wennergren. 1995. Connecting landscape patterns to ecosystem and population processes. Nature, 373:299-302.

Krummel, J.R., R.H. Gardner, G. Sugihara, R.V. O'Neill and P.R. Coleman. 1987. Landscape patterns in a disturbed environment. Oikos, 48:321-324.

Lamberson, R.H., R. McKevley, B.R. Noon and C. Voss. 1992. A dynamic analysis of northern spotted owl viability in a fragmented forest landscape. Conservation Biology, 6:505-512.

Lamberson R.H. and J. Carroll. 1993. Thresholds for Persistence in territorial species. In Topics in Biomathematics: Proceedings of the II International Conference on Mathematical Biology, I. Barbieri, E. Grassi, G. Pallotti and P. Pettazzoni (eds.), pp. 55-62. World Scientific Publishing Co., Singapore. 
Lamberson, R.H., B.R. Noon, C. Voss and K.S. McKelvey. 1994. Reserve design for territorial species: The effects of patch size and spacing on the viability of the northern spotted owl. Conservation Biology, 8:185-195.

Lande, R. 1987. Extinction thresholds in demographic models of territorial populations. American Naturalist, 130:624-635.

Levins, R. 1969. Some demographic and genetic consequences of environmental heterogeneity for biological control. Bulletin of the Entomological Society of America, 15:237-240.

Mandelbrot, B.B. 1982. The Fractal Geometry of Nature. Freeman, New York.

McKelvey, K., B.R. Noon and R.H. Lamberson. 1993. Conservation planning for species occupying fragmented landscapes: The case of the northern spotted owl. In Biotic Interactions and Global Change, Kareiva P.M., Kingsolver J.L., and Huey R.B. (eds.), pp. 424-450. Sinauer Associates Inc., Massachusetts.

Milne, B.T. 1988. Measuring the fractal geometry of landscapes. Applied Mathematics and Computation, 27:67-79.

Molofski, J. 1994. Population dynamics and pattern fluctuations in theoretical populations. Ecology, 75:30-39.

Nee, S. and R.M. May. 1992. Dynamics of metapopulations: habitat destruction and competition coexistence. Journal of Animal Ecology, 61:37-40.

Noon, B.R. and K.S. McKelvey. 1996. A common framework for conservation planning: Linking individual and metapopulation models. In Metapopulations and Wildlife Conservation, D.R. McCullough (ed.), pp. 139-166. Island Press, Washington, D.C.

Palmer, M.W. 1988. Fractal geometry: a tool for describing spatial patterns of plant communities. Vegetatio, 75:91-102. 
Peitgen, H. and D. Saupe. 1988. The Science of Fractal Images. Springer-Verlag, New York.

Saupe, D. 1988. Algorithms for random fractals. In The Science of Fractal Images, H. Peitgen and D. Saupe (eds.), pp.71-136. Springer-Verlag, New York.

Scheuring, I. 1991. The fractal nature of vegetation and the species-area relationship. Theoretical Population Biology, 39:170-100.

Silverton, J., S. Holtier, J. Johnson and P. Dale. 1992. Cellular automata models of interspecific competition for space - the effect of pattern on process. Ecology, 80:527-534.

Sole, R.V. and S.C. Manrubia. 1995. Are rainforests self-organized in a critical state? Journal of Theoretical Biology, 173:31-40.

Stone, L. 1995. Biodiversity and habitat destruction: a comparative study of model forest and coral reef systems. Proceedings of the Royal Society of London, 262:381388.

Tillman, D., R.M. May, C.L. Lehman and M.A. Nowak. 1994. Habitat destruction and the extinction debt. Nature, 371:65-66.

Wiggins, S. 1990. Introduction to applied nonlinear dynamical systems and chaos. Springer-Verlag: New York. 


\section{Chapter 3}

\section{The effects of habitat destruction in finite landscapes: A chain-binomial metapopulation model}

Interestingly, according to modern astronomers, space is finite. This is a very comforting thought-particularly for people who can never remember where they have left things.

-Woody Allen

\subsection{Introduction}

Many species living in fragmented habitats exist as an assemblage of local populations occupying distinct patches that are connected, to varying degrees, by dispersal (Levins 1969; Hanski and Gilpin 1997; Hanski 1999). Ecologists call such assemblages metapopulations (Hanski and Simberloff 1997). To persist, a metapopulation must balance the extinction of local populations with the colonization of empty patches (Hanski 1999).

A major threat to metapopulation persistence is the destruction of habitat due

\footnotetext{
${ }^{1}$ This chapter been submitted to Oikos for publication.
} 
to human activities. Habitat destruction affects the balance between colonization and extinction rates by reducing the number of suitable patches, i.e. patches that are capable of supporting a local population. As the number of suitable patches decreases, stochastic fluctuations in colonization and local extinctions render the metapopulation increasingly vulnerable to global extinction (Lande et al. 1998).

A common approach to investigating the effects of habitat destruction on the abundance and persistence of a species has been to use metapopulation models (e.g. Levins 1969; Lande 1987; Nee and May 1992; Lawton et al. 1994; Nee 1994; Tillman et al. 1994; Stone 1995; Gyllenberg and Hanski 1997; Bascompte and Sole 1996, 1998; Hill and Caswell 1999). These models have shown that the proportion of suitable patches occupied declines as habitat is destroyed, and that a metapopulation cannot persist when the fraction of suitable patches in the landscape falls below a critical threshold.

Most of these models assume that the landscape consists of an infinite number of patches, and describe the landscape in terms of the proportion of these patches that are occupied. While such models may provide a good approximation in landscapes consisting of hundreds of patches, they may fail badly when the number of suitable patches is small (Nisbet and Gurney 1982; Hanski 1999).

To study the effects of habitat destruction in small finite landscapes, we developed a stochastic model that describes the landscape in terms of the number, rather than the proportion of occupied patches. The model specifies probabilities of transition from any number, to any other number of occupied patches. The change in the number of occupied patches has a binomial distribution that can be specified in terms of colonization and extinction probabilities. Similar models have been historically used in epidemiology (Bailey 1957), and have recently been used by Klok and De Roos (1998) and Richards et al. (1999). Because dynamics are a sequence of binomial probabilities, the models are called chain-binomial. We will refer to our model as a chain binomial metapopulation (CBM) model. 
In the CBM model, the state of the metapopulation at time $t$ is described by the number, $n$, of occupied patches. The state space is the set of all integers $0 \leq n \leq S$, where $S$ is the number of suitable patches in the landscape. The output of the model is a probability distribution vector $\mathbf{x}(t)$, where $x_{i}(t)$ is the probability that $n=i$. The $\mathrm{CBM}$ model is an absorbing Markov chain, where extinction is the absorbing state.

In this paper, we analyze the CBM model to study the effects of habitat destruction in small landscapes. We use methods from stochastic process theory to examine how quasi-equilibrium patch occupancy frequencies, mean extinction times, and persistence probabilities vary as a function of the number of suitable patches and the ability of propagules to search for suitable patches. We also describe a method for parameterizing the CBM model from time series data, and then use this method to study how the frequency of parasitoid outbreaks affects extinction times of a host butterfly population.

\subsection{Model Description}

The landscape contains $N$ patches, $S$ of which are suitable, where $S \leq N$. Only suitable patches are capable of supporting a local population. Suitable patches become empty when the population occupying them goes extinct, while empty patches become occupied after being colonized and developing their own populations. Habitat destruction decreases the number of suitable patches within the landscape.

We model the dynamics of the metapopulation as a two-part process. The first part describes extinction within patches, while the second part describes the colonization of unoccupied patches. For each of these processes we derive a transition matrix whose elements give the probability that $i$ patches will be occupied after a unit of time given that $j$ patches are currently occupied. 


\subsubsection{Extinction matrix}

The number of occupied patches decreases when one or more local populations go extinct. A local population goes extinct with probability $\delta$ and survives with probability $1-\delta$. If $n$ patches are occupied before the extinction process, then the number of patches occupied after extinction is a binomial random variable with parameters $(n, \delta)$. The probability, $m_{\delta}(i, j)$, that $i$ patches are occupied after extinction given that $j$ patches were occupied before extinction is

$$
m_{\delta}(i, j)= \begin{cases}\left(\begin{array}{c}
j \\
i
\end{array}\right)(1-\delta)^{i} \delta^{j-i} & \text { if } i \leq j \\
0 & \text { otherwise }\end{cases}
$$

for $i, j \leq S$. Extinction is represented by a $(S+1) \times(S+1)$, upper triangular matrix $\mathbf{M}_{\delta}$ whose $(i, j)$ element is $m_{\delta}(i, j)$. For example, when $S=3$ and $\delta=0.1$,

$$
\mathbf{M}_{\delta}=\left(\begin{array}{llll}
1.000 & 0.100 & 0.010 & 0.001 \\
0 & 0.900 & 0.180 & 0.027 \\
0 & 0 & 0.810 & 0.243 \\
0 & 0 & 0 & 0.729
\end{array}\right)
$$

\subsubsection{Colonization matrix}

The number of occupied patches increases when one or more empty patches are colonized. An empty patch is colonized with probability $P_{c}$ and remains uncolonized with probability $1-P_{c}$. If $n$ patches are occupied before the colonization process, then the number of patches occupied after colonization is a binomial random variable with parameters $\left(n, P_{c}\right)$. The probability that $n$ increases from $j$ to $i$ is the probability that $i-j$ patches are colonized, where $i>j$. Thus the probability $m_{c}(i, j)$ that $i$ patches 
are occupied after colonization given $j$ patches were occupied before colonization is

$$
m_{c}(i, j)= \begin{cases}\left(\begin{array}{c}
S-j \\
i-j
\end{array}\right) P_{c}^{i-j}\left(1-P_{c}\right)^{S-i} & \text { if } i \geq j \\
0 & \text { otherwise }\end{cases}
$$

for $i, j \leq S$. Colonization is thus represented by an $(S+1) \times(S+1)$ lower triangular matrix, $\mathbf{M}_{c}$, whose $(i, j)$ element is $m_{c}(i, j)$.

We assume that colonization is a Poisson process, where the probability that an unoccupied patch is colonized is

$$
\begin{aligned}
P_{c} & =P[\geq 1 \text { offspring arrives at patch }] \\
& =1-\exp (-f(n))
\end{aligned}
$$

where $f(n)$ is the mean number of offspring arriving at each patch in the landscape, which depends on the number of occupied patches producing propagules. If propagules disperse randomly, the expected number of propagules arriving at a patch is

$$
f(n)=\frac{\beta n}{N}
$$

where $\beta$ is the number of dispersing propagules produced by each occupied patch. The parameter $\beta$ measures both reproductive output and colonization ability. Colonization depends on $N$ because propagules are dispersed over both suitable and unsuitable patches. An example of the colonization matrix for $b=0.5, S=3, N=6$ is

$$
\mathbf{M}_{c}=\left(\begin{array}{llll}
1.000 & 0 & 0 & 0 \\
0 & 0.847 & 0 & 0 \\
0 & 0.147 & 0.847 & 0 \\
0 & 0.006 & 0.154 & 1.000
\end{array}\right)
$$


Equation (3.5) assumes that the dispersal is random. Later in Section 2.4 we generalize $f(n)$ to incorporate propagule searching ability.

\subsubsection{CBM Model}

The transition from $t$ to $t+1$ requires both colonization and extinction. The transition matrix is

$$
\mathrm{A}=\mathrm{M}_{\delta} \mathbf{M}_{\mathrm{c}}
$$

A is a nonnegative column stochastic matrix of dimension $(S+1) \times(S+1)$, whose elements, $a_{i j}$, give the probability that $i$ patches are occupied at time $t+1$ given that $j$ patches were occupied at time $t$. Changing the order of the matrix multiplication (i.e. $\mathbf{M}_{\mathbf{c}} \mathbf{M}_{\delta}$ ) changes when you sample the metapopulation, after extinction or after colonization, but does not substantially change the long-term behavior of the model. An example of the matrix A, using the example matrices (3.2) and (3.6) from above, is shown below

$$
\mathbf{A}=\left(\begin{array}{llll}
1.000 & 0.086 & 0.009 & 0.001 \\
0 & 0.789 & 0.157 & 0.027 \\
0 & 0.121 & 0.723 & 0.243 \\
0 & 0.005 & 0.112 & 0.729
\end{array}\right)
$$

Note that state 1 (global extinction) is absorbing and that the largest probabilities lie on the diagonal of $\mathbf{A}$.

Figure 3.1 shows surface plots of A for larger values of $S$. When $\delta$ is small (fig. 3.1a) transition probabilities are highest near the diagonal of $\mathbf{A}$ and drop off sharply as you move away from the largest value in each column. As $\delta \rightarrow 1$ (fig 3.1b.), however, the largest transition probabilities lie increasingly above the diagonal of $\mathbf{A}$ and their distribution in each column becomes more spread out. 


\section{Given $j$ patches occupied at time $\mathrm{t}$}

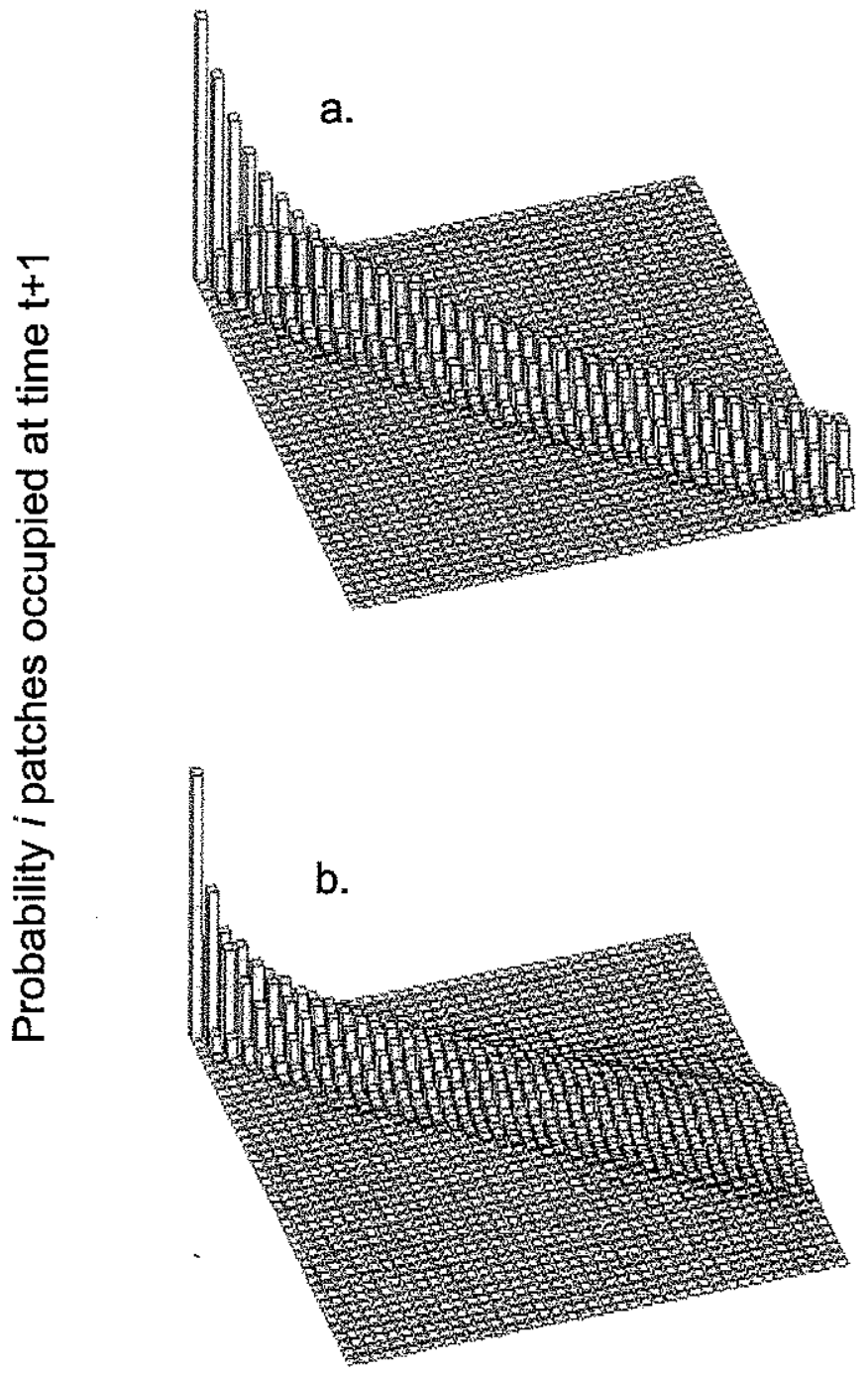

Figure 3.1: Graphic representation of the transition matrix A for the case $S=40$, $N=100$ and $\beta=0.5$. The value of each element $a_{i, j}$ is represented by the height of the bar in column $j$ row $i$. a. Transition matrix for $\delta=0.05$. b. Transition matrix for $\delta=0.4$. 


\subsubsection{Search Ability}

The model so far assumes that propagules passively accept the conditions (suitable or not) of the first patch they sample. Plant seeds may do so, but many species produce propagules that can sample many patches in search for unoccupied suitable space before dying. We can modify equation (3.5) for $f(n)$ in this case by assuming that a propagule that lands in an occupied or unsuitable patch, leaves that patch and samples the landscape again until it either finds an empty suitable patch, or dies. The search process is assumed to occur on a fast time scale within the colonization process.

On the first try, the mean number of propagules arriving at each patch is $\beta n / N$. Of these, a fraction

$$
\frac{n+(N-S)}{N}
$$

land in occupied or unsuitable patches. Of these, a fraction $\phi$ survive $(0 \leq \phi \leq 1)$, and try again. The mean number of propagules arriving at each patch who are on their second attempt is then

$$
\frac{\beta n}{N} \phi\left(\frac{n+(N-S)}{N}\right)
$$

Similarly, the mean number of propagules arriving at each patch who are on their third attempt is given by

$$
\frac{\beta n}{N} \phi\left(\frac{n+(N-S)}{N}\right) \phi\left(\frac{n+(N-S)}{N}\right)
$$

Thus the total number of propagules arriving at each patch within the colonization interval is

$$
\begin{aligned}
f(n) & =\frac{\beta n}{N}+\phi \frac{\beta n}{N}\left(\frac{n+(N-S)}{N}\right)+\phi^{2} \frac{\beta n}{N}\left(\frac{n+(N-S)}{N}\right)^{2}+\cdots \\
& =\frac{\beta n}{N-\phi(n+N-S)}
\end{aligned}
$$


Note that (3.5) is a special case of (3.12) with $\phi=0$.

The average number of patches that a propagule visits depends on the survival parameter $\phi$ and the number of suitable and unsuitable patches

$$
E \text { (Number of searches })=\frac{1}{1-\phi q}
$$

where $q=1-\frac{(S-n)}{N} \leq 1$. Thus there are fewer searches when $n$ is small compared to $S$ because the propagules have a better chance, on each try, of finding an empty suitable patch. As more habitat is destroyed ( $S$ decreases), the average number of searches increases because the probability of landing in an unsuitable patch increases.

\subsubsection{Model Analysis}

An example of a single stochastic realization of the model specified by $\mathbf{A}$ is shown in figure 3.2. The number of occupied patches fluctuates, and in this example the population avoids global extinction for 1000 iterations. The probability distribution for the number of occupied patches satisfies

$$
\mathbf{x}(t+1)=\mathbf{A} \mathbf{x}(t)
$$

where $x_{i}(t)$, is the probability that $i-1$ patches are occupied at time $t$. Figure fig:C3f3 shows an example of $\mathbf{x}(t)$ as a function of time, starting from an initial condition in which all suitable patches are occupied. Note that as $t \rightarrow \infty$, the probability that the metapopulation goes extinct $\rightarrow 1$.

The state $n=0$ is absorbing; hence the matrix $\mathbf{A}$ can be rewritten in the form

$$
\mathbf{A}=\left(\begin{array}{l|l}
1 & \mathbf{e} \\
\hline 0 & \mathbf{T}
\end{array}\right)
$$

where $\mathbf{e}$ is a $1 \times S$ row vector of extinction probabilities. The matrix $\mathbf{T}$ describes 


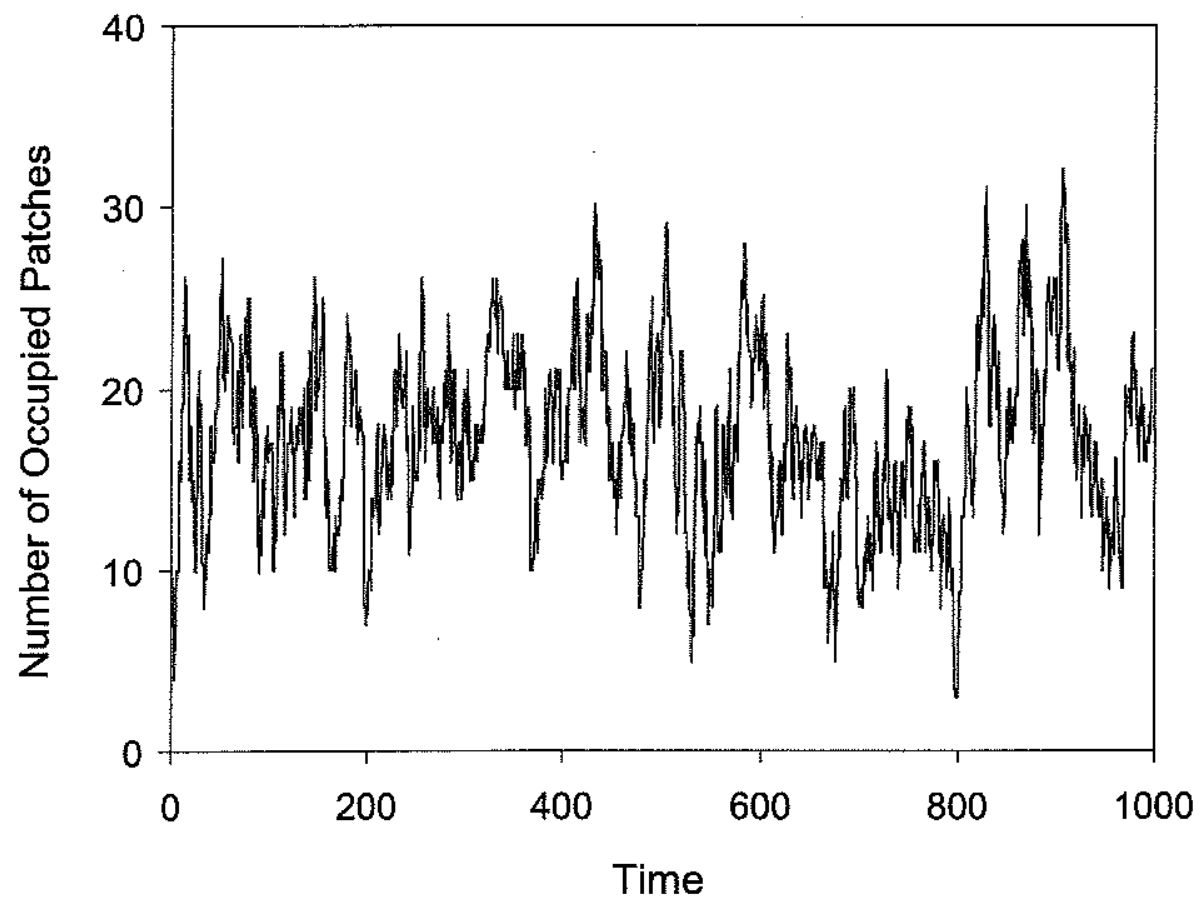

Figure 3.2: Stochastic realization of the number of occupied patches in a single landscape as a function of time. Parameter values are $S=35, N=100, \beta=0.5, \delta=0.1$ and $\phi=0$. 

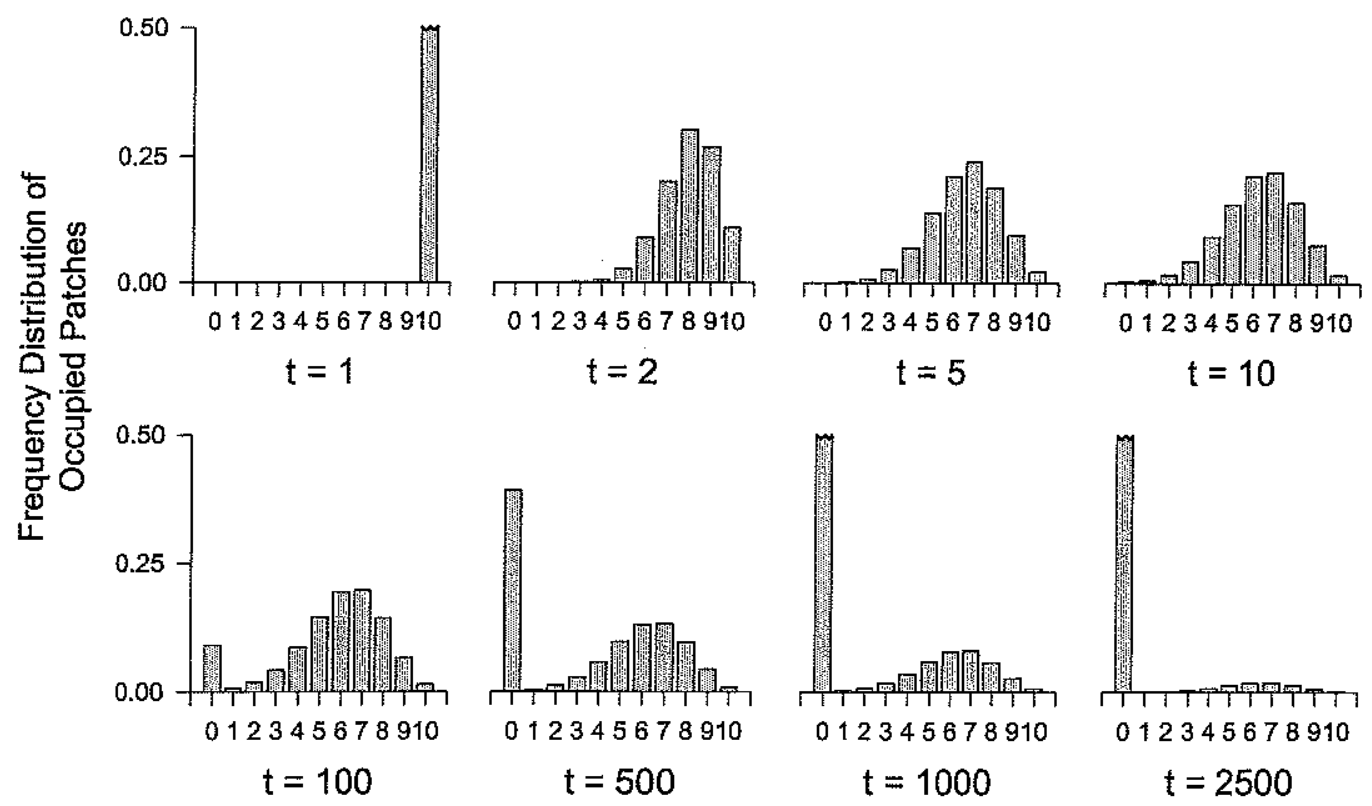

Figure 3.3: Time series of the distribution of landscapes with $n$ occupied patches starting from an initial condition in which all patches are occupied. Parameter values are $S=10, N=20, \beta=0.5, \delta=0.1$ and $\phi=0$.

transitions among the transient states $(i=2,3, \cdots, S+1)$. From the matrix $\mathbf{T}$ we can calculate the quasi-stationary distribution, the expected extinction time, and the probability that the metapopulation survives to time $t$.

The quasi-equilibrium distribution gives the probability distribution of the number of occupied patches, given that extinction has not yet happened and will not happen for a long time (Seneta 1966). Let $q_{i}$ denote the probability that $i$ patches are occupied $(i=1, \ldots, S)$. The distribution $\mathbf{q}$ can be calculated from the right and left eigenvectors, $\mathbf{w}$ and $\mathbf{v}$, corresponding to the largest eigenvalue of $\mathbf{T}$ :

$$
q_{i}=w_{i} v_{i}
$$

The probability vector $\mathbf{q}$, whose elements are $q_{i}$, is normalized appropriately so that $\sum_{i} q_{i}=1$. The expected number of occupied patches, $\bar{n}$, in the quasi-equilibrium 
distribution is then

$$
\bar{n}=\sum_{i=1}^{S} i q_{i} .
$$

and the variance in $n$ is

$$
\sigma^{2}(n)=\sum_{i} i^{2} q_{i}-\left(\sum_{i} i q_{i}\right)^{2}
$$

Dividing the expected number of occupied patches by the number of suitable patches gives

$$
\hat{p}=\bar{n} / S
$$

where $\hat{p}$ is the expected proportion of suitable patches that are occupied, given that the metapopulation has not gone extinct. We will refer to $\hat{p}$ as the quasi-equilibrium frequency.

The expected time to extinction is determined from the fundamental matrix of $\mathbf{T}$, given by

$$
\mathbf{F}=(\mathbf{I}-\mathbf{T})^{-1}
$$

where $\mathbf{I}$ is the identity matrix (Kemeny and Snell 1976). The expected extinction time given that $j$ patches are currently occupied, $\tau_{j}$, is calculated by summing the $j$ th column of $\mathbf{F}$

$$
\tau_{j}=\sum_{i} f_{i j}
$$

The expected extinction time for an initial state selected at random from the quasistationary distribution is

$$
\bar{\tau}=\sum_{i=1}^{S} q_{i} \tau_{i} .
$$

We will refer to $\bar{\tau}$ as the mean expected extinction time of the metapopulation.

Consider a metapopulation initially occupying $j$ patches. We denote by $l_{j}(x)$ the 
probability that it persists to "age" $x$. Let $\left(\mathbf{T}^{x}\right)_{i j}$ be the $(i, j)$ entry of $\mathbf{T}^{x}$. Then

$$
l_{j}(x)=\sum_{i}\left(\mathbf{T}^{x}\right)_{i j}
$$

The expected probability that the metapopulation persists to time $x$ for an initial state selected at random from the quasi-stationary distribution is

$$
\bar{l}(x)=\sum_{j=1}^{S} q_{j} l_{j}(x)
$$

We will refer to $\bar{l}(x)$ as the mean persistence probability to time $x$.

\subsection{Results: The Effect of Habitat Destruction}

In this section we illustrate the effect of habitat destruction on the quasi-equilibrium frequency $\hat{p}$ and the expected extinction time $\bar{\tau}$. We also show how changing the searching ability of propagules, $\phi$, effects $\hat{p}$ and $\bar{\tau}$. Finally, we show how the mean persistence probability $\bar{l}(x)$ varies as a function of time and the number of suitable patches.

Figure 3.4 shows how $\hat{p}$ depends on the number of suitable patches $S$. Reductions in $S$ cause $\hat{p}$ to decline. At very low values of $S$, however, $\hat{p}$ increases, because $\hat{p}$ is the expected patch occupancy given that the metapopulation has not gone extinct. Thus in the extreme case, if there is only a single patch and the population is not extinct, that patch must be occupied and $\hat{p}=1$.

Figure 3.5 illustrates how quasi-equilibrium frequencies are affected by the propagule searching parameter $\phi$. Increasing $\phi$ increases both $\hat{p}$ and the amount of habitat destruction the metapopulation can tolerate (fig. 3.5a). Increasing $\phi$ also decreases the variance, $\sigma^{2}(n)$, of the quasi-equilibrium distribution (fig. $3.5 \mathrm{~b}$ ). Thus patch occupancy frequencies for a species with good searching abilities are less variable than a species with poor searching abilities. 


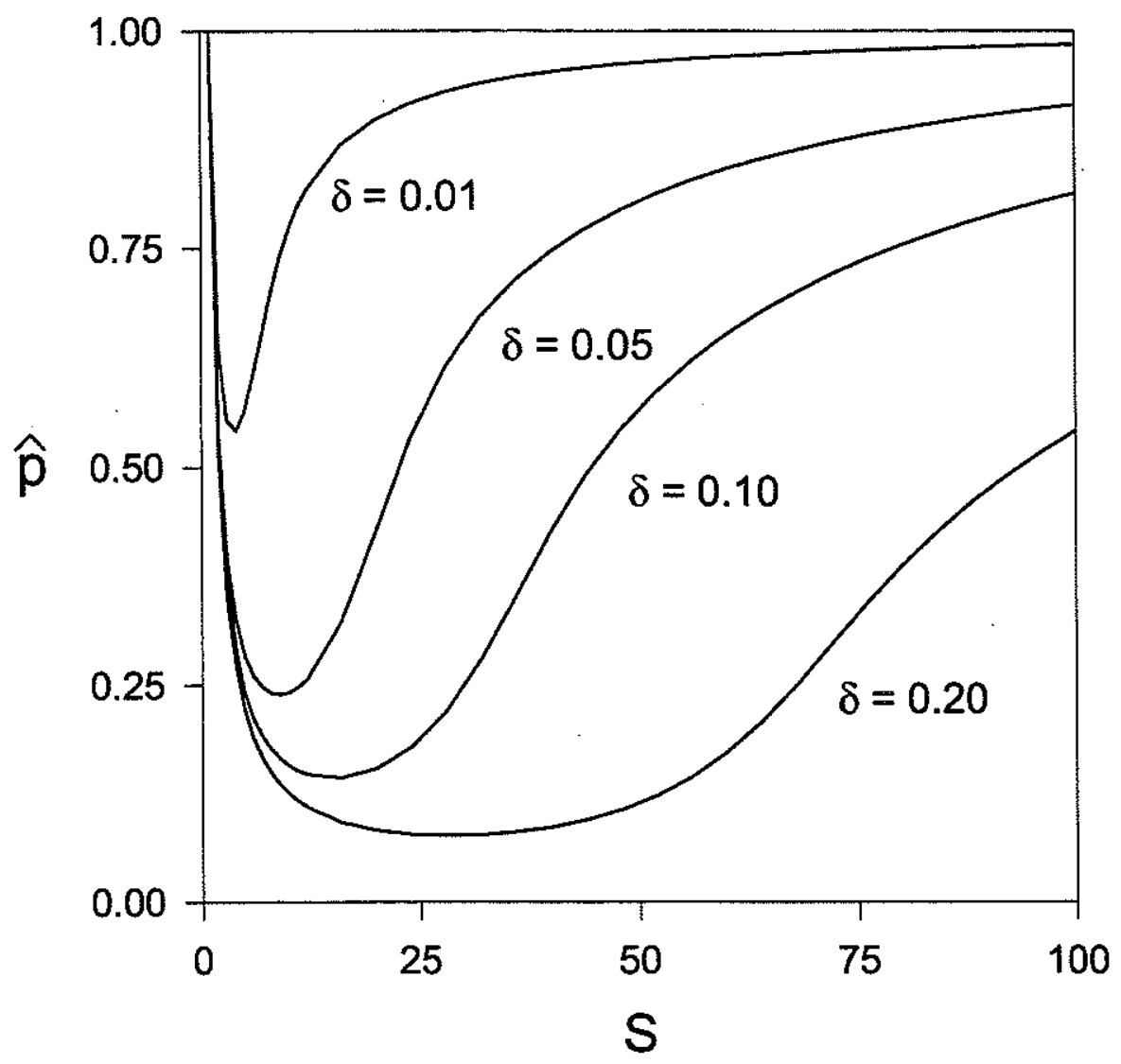

Figure 3.4: Quasi-equilibrium frequency, $\hat{p}$, as a function of the number of suitable patches, $S$. The landscape size is $N=100$. Parameter values are $\beta=0.5$ and $\phi=0$. 

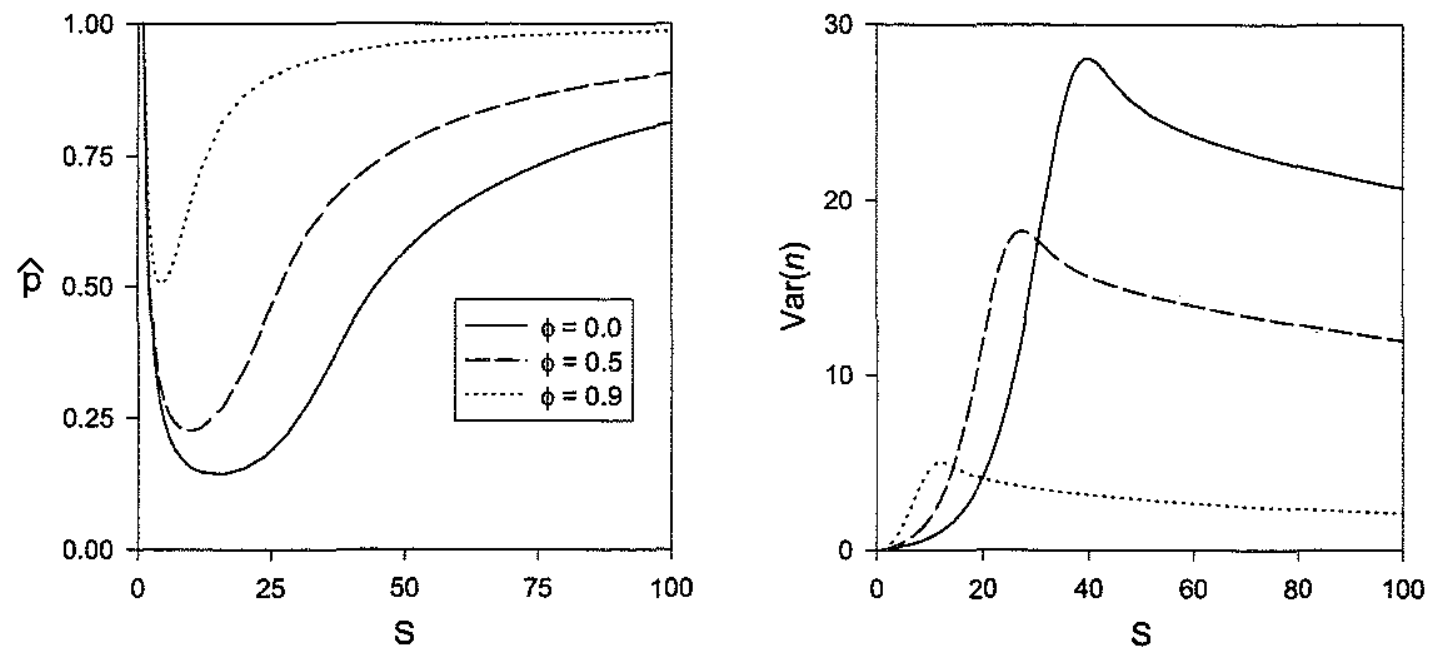

Figure 3.5: Varying the searching ability of propagules. $a$. The effect of the search parameter $\phi$ on quasi-equilibrium frequencies, $\hat{p}$. $b$. The effect of the search parameter, $\phi$, on the variance of the quasi-stationary distribution, $\sigma(n)^{2}$. Parameter values are $\beta=0.5, \delta=0.1$ and $N=100$.

Figures 3.6 shows the expected time to extinction, $\tilde{\tau}$, as a function of the number of suitable patches. When $\tilde{\tau}$ is plotted on a logarithm scale, the slope is a convex increasing line (fig 3.6a), hence $\bar{\tau}$ increases greater than exponentially with $S$. Plotting $\bar{\tau}$ on a linear scale (fig 3.6b.) illustrates the large effect that small changes in $S$ have on $\bar{\tau}$. There is a threshold number of patches $S$ at which $\bar{\tau}$ increases abruptly from small values to values so large as to be effectively infinite. The consequences of this effect are especially important when $\bar{\tau}$ is small (note, the point where the lines in fig. $6 \mathrm{~b}$ appear to meet the $\mathrm{x}$ axis depends on the scale of the $\mathrm{y}$ axis). Figure 3.6 also shows that increasing the search parameter $\phi$ increases the expected time to extinction. Given 2 species with similar values of $\beta$ and $\delta$ but different values of $\phi$, the species with the better searching ability will persist longer for a given value of $S$, and can tolerate a greater loss of suitable habitat.

Figure 3.7 illustrates how the mean persistence probability to time $x, \bar{l}(x)$ depends on $S$. In general, $\bar{l}(x)$ is always a monotonically decreasing function of time, however, the rate of decrease is highly sensitive to changes in $S$. For example when $N=100$, 
a.

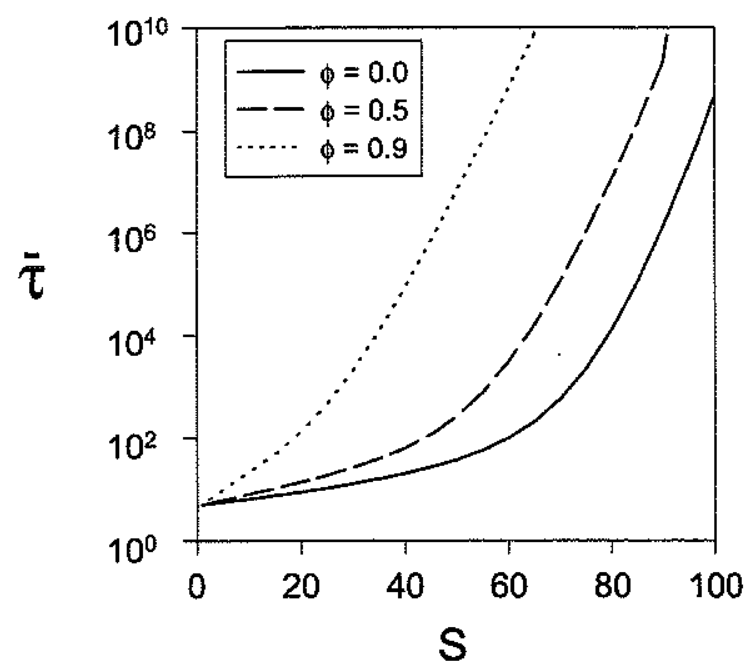

b.

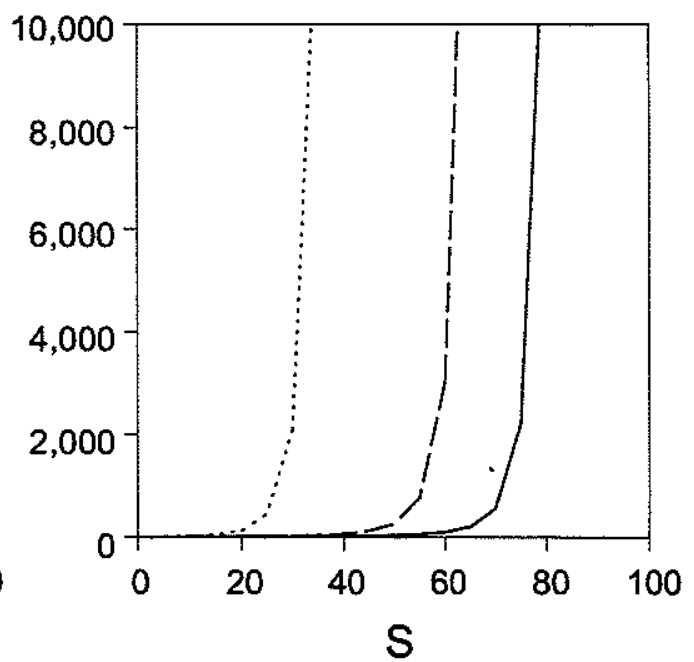

Figure 3.6: The effect of habitat destruction on extinction times. a. Expected extinction time $\bar{\tau}$ as a function of the number of suitable patches, $S$, and the search parameter $\phi . \bar{\tau}$ is plotted on a logarithmic scale $b$. Same as fig. 6a except $\bar{\tau}$ is now plotted on a linear scale. Parameter values are $\beta=0.5, \delta=0.2$, and $N=100$.

$\beta=0.5, \delta=0.2$, and $\phi=0.5$, a reduction in $S$ form 60 to 50 reduces the chance of surviving at least 500 years from $85 \%$ to $14 \%$. Thus in terms of persistence, there is a threshold range of habitat destruction over which small changes in $S$ dramatically affect the probability that the metapopulation persists for a long period of time.

\subsection{Parameter Estimation}

In this section we describe a method for estimating $\beta$ and $\delta$ from time series data. We focus on the case where $\phi=0$ (i.e. propagules have no searching ability). The same basic principles apply for species in which $\phi>0$, however, it may not always be possible to get an accurate estimate of $\beta$ when $\phi$ is unknown. One way around this problem is to specify a value of $\phi$ first. If information is available on the average number of patches visited by propagules, then equation (3.13) can be used to calculate $\phi$. Otherwise, $\beta$ and $\delta$ can be estimated for a range of likely $\phi$ values. 


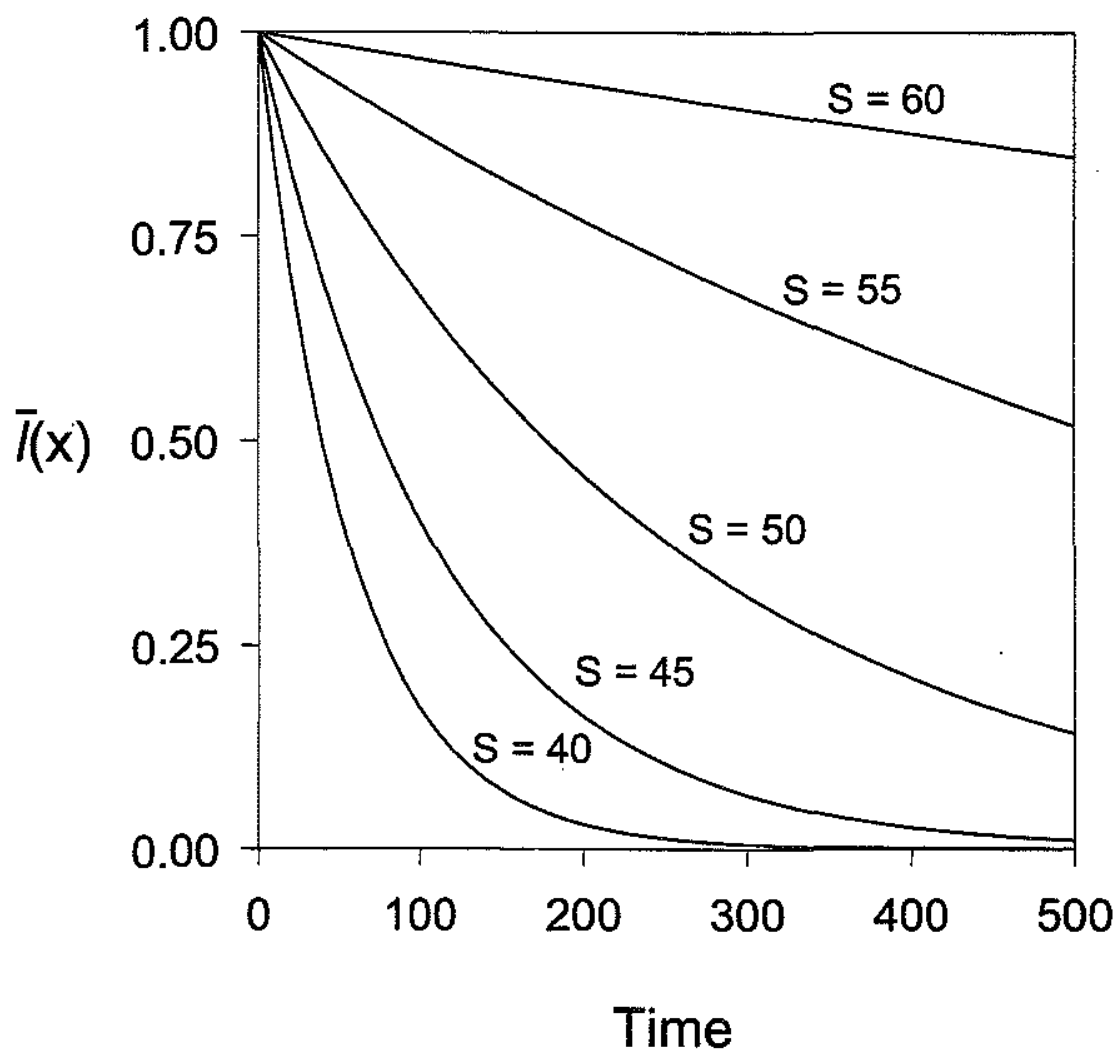

Figure 3.7: The mean persistence probability $\bar{l}(x)$ as a function of time and the number $S$ of suitable patches. Parameters values are $\beta=0.5, \delta=0.2, \phi=0.5$, and $N=100$. 


\subsubsection{Likelihood function}

We want to estimate parameters for real metapopulations. Suppose data are available in the form of a three-way contingency table, where the $(i, j, k)$ entry of the table is the number of patches making the transition from state $i$, to fate $j$, at time $k$. The table layout for a single time interval (i.e. $k=1$ ) is shown below

\begin{tabular}{||c|c|c||}
\hline & $O(t)$ & $U(t)$ \\
\hline$O(t+1)$ & $\mathrm{A}$ & $\mathrm{C}$ \\
\hline$U(t+1)$ & $\mathrm{B}$ & $\mathrm{D}$ \\
\hline
\end{tabular}

where $O(t)$ and $U(t)$ are the number of occupied and unoccupied patches at time $t$, and the letters $A, B, C$, and $D$ represent the number of patches that fall into each of the transition categories, and $N=A+B+C+D$.

We can estimate $\beta$ and $\delta$ by maximum likelihood. The probability that a patch undergoes a particular transition is obtained by summing together the probabilities of all possible events that could produce the transition. For example, the probability that an occupied patch becomes unoccupied during one time interval is

$$
\begin{aligned}
P[O(t+1) \mid U(t)] & =\delta\left(1-P_{c}\right)+\delta P_{c} \delta\left(1-P_{c}\right)+\left(\delta P_{c}\right)^{2} \delta\left(1-P_{c}\right)+\ldots \\
& =\frac{\delta\left(1-P_{c}\right)}{1-\delta P_{c}}
\end{aligned}
$$

where $P_{c}=1-\exp (-b O(t) / N)$. The first term on the right side of (3.25) is the probability the patch was disturbed (i.e. local population went extinct) and not recolonized. The second term is the probability that the patch was disturbed, recolonized, disturbed, and not recolonized; and so on. The other transition probabilities are calculated similarly and are shown in the table below

\begin{tabular}{||c|c|c||}
\hline & $O(t)$ & $U(t)$ \\
\hline$O(t+1)$ & $\frac{1-\delta}{1-\delta P_{c}}$ & $\frac{P_{c}(1-\delta)}{1-\delta P_{c}}$ \\
\hline$U(t+1)$ & $\frac{\delta\left(1-P_{c}\right)}{1-\delta P_{c}}$ & $\frac{1-P_{c}}{1-\delta P_{c}}$ \\
\hline
\end{tabular}


Suppose the transition data is collected over $k$ time intervals. The likelihood function for $\beta$ and $\delta$ given the observed data is

$$
L(\beta, \delta \mid \text { Data })=\prod_{t=1}^{k} \gamma\left(\frac{1-\delta}{1-\delta P_{c}}\right)^{A(t)}\left(\frac{\delta\left(1-P_{c}\right)}{1-\delta P_{c}}\right)^{B(t)}\left(\frac{P_{c}(1-\delta)}{1-\delta P_{c}}\right)^{C(t)}\left(\frac{1-P_{c}}{1-\delta P_{c}}\right)^{D(t)}
$$

where $\gamma$ is an arbitrary constant, $\theta$ denotes the unknown parameters $(\beta$ and $\delta$ in this case) and $\Sigma$ denotes the observed data. Statistical calculations utilize the loglikelihood of (3.27) given by

$$
\begin{aligned}
\ln L(\beta, \delta \mid \text { Data })= & \sum_{t=1}^{k} A(t) \ln (1-\delta)+B(t) \ln \left(\delta\left(1-P_{c}\right)+\ldots\right. \\
& C(t) \ln \left(P_{c}(1-\delta)\right)+D(t) \ln \left(1-P_{c}\right)-N \ln \left(1-\delta P_{c}\right)
\end{aligned}
$$

The value of $\beta$ and $\delta$ that maximizes (3.28), is the maximum likelihood estimate of the model parameters given the observed data (Edwards 1992).

\subsubsection{An Example}

Lei and Hanski (1997) and Hanski (1999) recorded five years of patch occupancy data (1993 - 1997) on the Glanville fritillary butterfly Meltiaea cinxia in the Åland islands (SW Finland). The data is in the form of yearly spatial maps identifying the location of occupied and unoccupied patches in a landscape of 63 patches (see fig. 12.9 in Hanski [1999]). We reorganized this data into a three-way contingency table, which is shown in figure $3.8 \mathrm{a}$.

Figure $3.8 \mathrm{~b}$ shows how the number of patches occupied by $M$. cinxia and a specialist parasitoid wasp Cotesia melitaearum varied as a function of time in the Åland landscape. The wasp increases the mortality of $M$. cinxia by attacking the larvae of its butterfly host. Between 1993-1995 there was a declining trend in the number of patches occupied by $M$. cinxia due to a high incidence of $C$. melitaearum (Lei and Hanski 1997). In 1995, however, C. melitaearum became nearly extinct in the 
a.

\begin{tabular}{|c|c|c|c|}
\hline & $\mathrm{O}(\mathrm{t})$ & $\mathrm{U}(\mathrm{t})$ & Total \\
\hline $\mathrm{O}(\mathrm{t}+1)$ & 24 & 2 & 26 \\
\hline $\mathrm{U}(\mathrm{t}+1)$ & 8 & 29 & 37 \\
\hline Total & 32 & 31 & 63 \\
\hline
\end{tabular}

\begin{tabular}{|c|c|c|c|}
\hline & $O(t)$ & $U(t)$ & Total \\
\hline$O(t+1)$ & 10 & 4 & 14 \\
\hline$U(t+1)$ & 16 & 33 & 49 \\
\hline Total & 26 & 37 & 63 \\
\hline
\end{tabular}

\begin{tabular}{|c|c|c|c|}
\hline & $O(t)$ & $U(t)$ & Total \\
\hline$O(t+1)$ & 9 & 6 & 15 \\
\hline$U(t+1)$ & 5 & 43 & 48 \\
\hline Total & 14 & 49 & 63 \\
\hline
\end{tabular}

\begin{tabular}{|c|c|c|c|}
\hline & $1996-1997$ \\
\hline$O(t+1)$ & 13 & $8(t)$ & Total \\
\hline$U(t+1)$ & 2 & 40 & 21 \\
\hline Total & 15 & 48 & 63 \\
\hline
\end{tabular}

b.

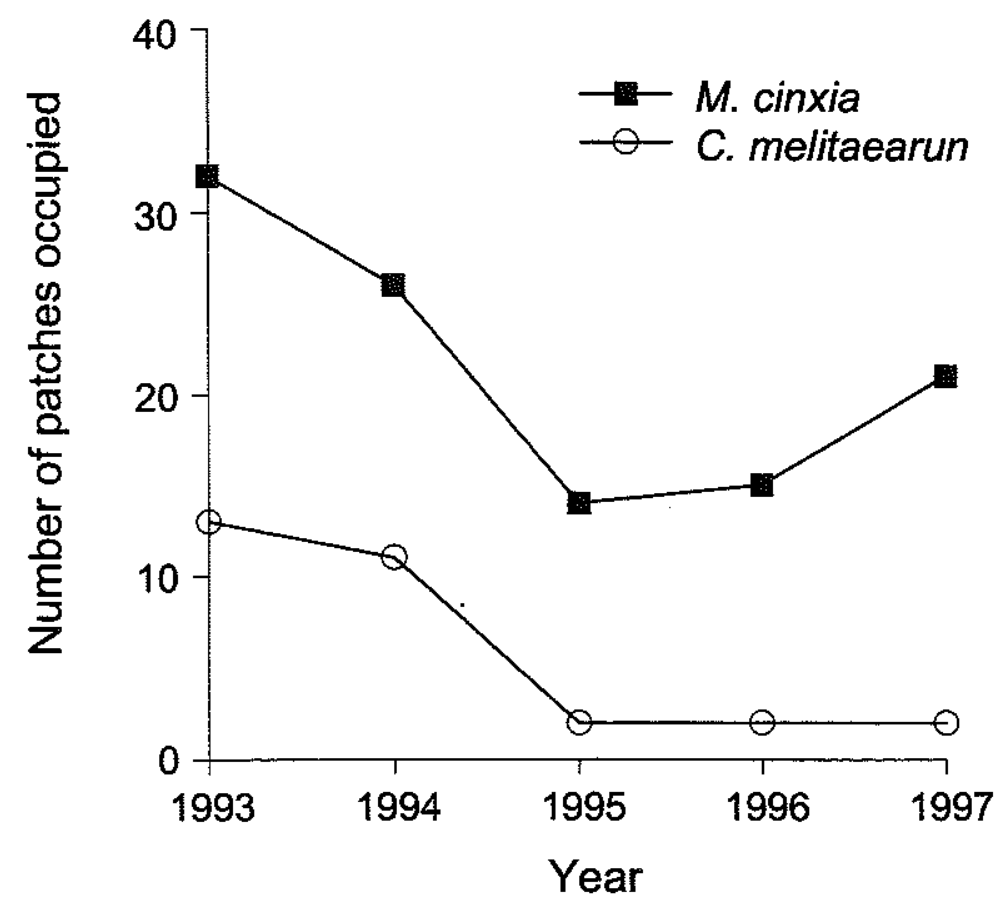

Figure 3.8: Patch transition data for a network of 63 patches in the Aland islands (SW Finland). a. A three-way contingency table showing the observed yearly patch transitions for the Glanville fritillary butterfly Meltiaea cinxia from 1993 - 1997. $b$. Number of patches occupied by the butterfly $M$. cinxia and its specialist parasitoid Cotesia melitaearum as a function of time. 


\begin{tabular}{c|ccc}
\hline \hline & $1993-1995$ & $1995-1997$ & $1993-1997$ \\
\hline$\beta$ & 0.3488 & 0.9198 & 0.6080 \\
$\delta$ & 0.4548 & 0.2826 & 0.4083 \\
$\bar{\tau}$ & 4.18 yrs. & $2.69 \times 10^{7}$ yrs. & 13.61 yrs. \\
\hline \hline
\end{tabular}

Table 3.1: Likelihood estimates of $\beta$ and $\delta$ based on time series data for the Glanville fritillary butterfly Meltiaea cinxia. Each column shows the estimated values of $\beta$ and $\delta$ for a specific set of observation years. The expected extinction times for the models specified by each set of parameter values are shown at the bottom of each column (see text for details).

landscape, allowing $M$. cinxia to recover between 1995-1997 (Hanski 1999).

To look at the effects of $C$. melitaearum on $M$. cinxia, we estimated $\beta$ and $\delta$ using data for 1993-1995 only (parasite outbreak), for 1995-1997 only (low parasite abundance), and for the entire data set (1993-1997). Table 3.1 lists parameter estimates for each time period. From these estimates we calculated three transition matrices, $\mathbf{A}_{o}$ (outbreak years), $\mathbf{A}_{n}$ (non-outbreak years) and $\mathbf{A}_{\text {all }}$ (all years); and calculated $\bar{\tau}$ for each matrix (Table 3.1). The expected extinction time in non-outbreak years is 6 million times larger than that in outbreak years. The expected extinction time for the entire data set, on the other hand, is only slightly larger than for the outbreak years. This pattern suggests that outbreaks of $C$. melitaearum devastate the $M$. cinxia metapopulation and that more than 2 years are required, following an outbreak, for the recovery of $M$. cinxia.

We studied how the frequency of parasite outbreaks affect extinction times by calculating the matrix

$$
\mathbf{A}_{F}=\mathbf{A}_{n}^{k-1} \mathbf{A}_{o}
$$

where $k-1$ is the number of years between outbreaks and $F=1 / k$ is the outbreak frequency. The expected extinction time specified by $\mathbf{A}_{F}\left(\bar{\tau}_{F}\right)$ is in units of $k$ years. To convert to a yearly time scale, $\bar{\tau}_{F}$ is multiplied by $k$

$$
\bar{\tau}_{F y}=k \bar{\tau}_{F} .
$$



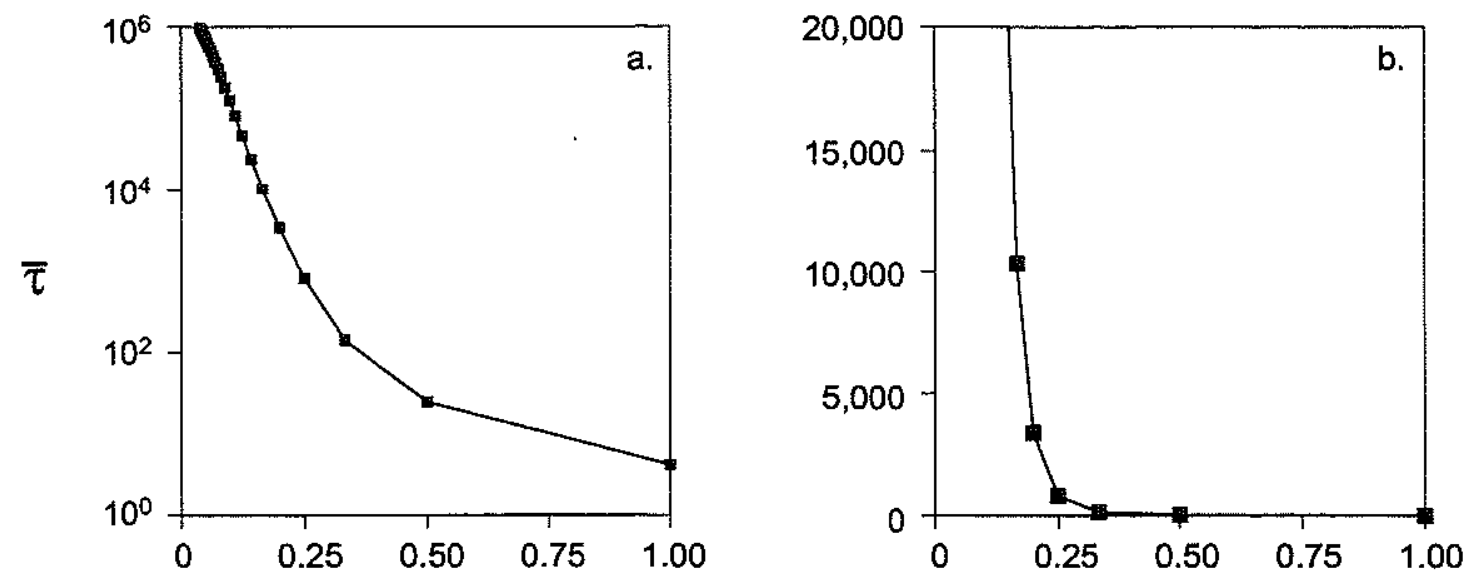

Parasitoid Outbreak Frequency

Figure 3.9: The expected extinction time of Meltiaea cinxia as a function of the frequency of Cotesia melitaearum outbreaks. $a . \bar{\tau}$ is plotted on a logarithmic scale $b$. $\bar{\tau}$ is plotted on a linear scale.

A plot of $\bar{\tau}_{F y}$ as a function of outbreak frequency is shown in figure 3.9. The extinction time of $M$. cinxia decreases greater than exponentially as the frequency of $C$. melitaearum increases, and has a threshold at $F \approx 0.25$ (fig. $3.9 \mathrm{~b}$ ). If the frequency of outbreaks occur once every four years (or less), then the likelihood that M. cinxia goes extinct in the near future is relatively certain. If outbreaks are spaced five years apart, however, then the expected time extinction time jumps abruptly to about 3000 years. Again we see a sharp threshold in $\bar{\tau}$, this time as a function of the frequency of a naturally occurring disturbance. Note that the curves in fig. 3.9 are probably a best case scenario, since we assumed outbreaks are evenly spaced in time and last only one year.

\subsection{Discussion}

Metapopulation models often describe the effects of habitat destruction on persistence by asssuming that colonization and extinction take place in an infinite landscape. 
Such models predict that the metapopulation either goes extinct or persists at a stable equilibrium, depending on the proportion of suitable habitat. These predictions, however, may fail badly when applied to landscapes consisting of only a small number of patches (Nisbet and Gurney 1982, Hanski 1999). In a finite landscape a metapopulation can go extinct simply because all subpopulations happen to go extinct at the same time. This is a metapopulation analog of demographic stochasticity, and has been termed colonization-extinction stochasticity by Hanski (1999). Colonizationextinction stochasticity becomes increasingly important as the size of the landscape and the number of suitable patches becomes small (Nisbet and Gurney 1982). To describe the dynamics of a finite landscape, a model must account for all possible fates of patches.

Quasi-equilibrium frequencies are a measure of occupied patch densities within suitable habitat given the metapopulation has not gone extinct. They are predicted to decline as the number of suitable patches, $S$, declines. At low values of $S$, however, $\hat{p}$ increases as $S$ declines. This phenomenon corresponds to situations where a scientists studies a metapopulation on a small spatial scale (only a few or even one suitable patch), or chooses regions to study based on the presence of the species. The latter might be common, since most biologists are reluctant to begin studying a species in a place where it is known to be extinct.

Several models (e.g. Lande 1987; Lamberson et al. 1994; With and King 1999) have shown that increasing the number of times migrants can search for suitable patches increases $\hat{p}$ and lowers the extinction threshold (i.e. the value of $h$ at which a metapopulation goes extinct). In the CBM model, increasing the search parameter $\phi$ not only increases $\hat{p}$ but also reduces the amount of variability in the landscape probability distribution (fig. $3.5 \mathrm{~b}$ ). A reduction in $\sigma^{2}(n)$ reduces the probability that a landscape will have zero occupied patches, thereby increasing the expected time to metapopulation extinction. This result is consistent with previous findings that species with better search abilities (as measure here by $\phi$ ) can tolerate more habitat 
destruction.

Mean extinction times are a greater than exponentially increasing function of $S$. This relationship means that even small amounts of habitat destruction dramatically reduce $\bar{\tau}$ (fig $6 \mathrm{~b}$ ). As the number of suitable patches decreases, there is a threshold response to habitat destruction in which $\bar{\tau}$ decreases abruptly from effectively infinite values (on an ecological time scale) to very small values. This threshold is also evident in terms of $\bar{l}(x)$, where the probability of persistence over a particular time interval $x$ can also decline drastically for small changes in $S$ (fig. 3.7). In general, $\bar{\tau}$ and $\bar{l}(x)$ are much more sensitive to changes in $S$ than $\hat{p}$, and thus provide a better assessment of the effects of habitat destruction in finite landscapes. Predictions of metapopulation viability based on $\hat{p}$ do not capture the true risk of global extinction in small finite landscapes as there is not the same threshold response to changes in $S$, as there is with $\bar{\tau}$ and $\bar{l}(x)$. Large underestimates in metapopulation persistence times can result by concentrating only on occupied patch frequencies.

The parameterization methods presented here are straight forward, and are applicable to any species with a metapopulation structure confined to a small network of patches. Once the CBM model is parameterized, it can used to predict how $\hat{p}, \bar{\tau}$, and $\vec{l}(x)$ are effected by a variety of processes, such as habitat loss, parasite outbreaks (as in the case of $M$. cinxia), habitat degradation, etc. As a large majority of the worlds threatened and endangered species are confined to small habitats, it is important to understand how they will respond to natural and human induced disturbances. The CBM model provides a simple but useful framework for studying these processes. 


\subsection{References}

Bailey, N.T.J. 1957. The Mathematical Theory of Infectious Diseases. - Charles Griffin \& Company LTD, London.

Bascompte, J. and Sole, R.V. 1996. Habitat fragmentation and extinction thresholds in spatially explicit models. - J. Anim. Ecol. 65:465-473.

Bascompte, J. and Sole, R.V. 1998. Effects of habitat destruction in a prey-predator metapopulation model. - J. Theor. Ecol. 195:383-393.

Edwards, A.W.F. 1992. Likelihood: Expanded Edition. - The Johns Hopkins University Press LTD, London.

Gyllenberg, M. and Hanski, I. 1997. Habitat deterioration, habitat destruction, and metapopulation persistence in heterogeneous landscapes. - Theor. Popul. Biol. 52:198-215.

Hanski, I. 1999. Metapopulation ecology. - Oxford University Press, Oxford.

Hanski, I. and Gilpin, M.E. 1997. Metapopulation Biology. - Acedemic Press, San Diego.

Hanski, I. and Simberloof, D. 1997. The metapopulation approach, its history, conceptual domain and application to conservation. - In: I. Hanski, I. and Gilpin M. (eds.), Metapopulation biology: ecology, genetics and evolution. Academic Press, London, pp. 5-26.

Hill, M. and Caswell, H. 1999. Habitat fragmentation and extinction thresholds on fractal landscapes. - Ecol. Letters 2:121-127.

Klok, C. and De Roos, A.M. 1998. Effects of habitat size and habitat quality on equilibrium density and extinction time of Sorex araneus populations. - J. Anim. Ecol. 67:195-209. 
Lamberson, R.H., Noon, B.R., Voss, C. and McKelvey, K.S. 1994. Reserve design for territorial species: The effects of patch size and spacing on the viability of the northern spotted owl. - Cons. Biol. 8:185-195.

Lande, R. 1987. Extinction thresholds in demographic models of territorial populations. - Am. Nat. 130:624-635

Lande, R., Engen, S. and Saether, B-E. 1998. Extinction times in finite metapopulation models with stochastic local dynamics. - Oikos 83:383-389.

Lawton, J.H., Nee, S., Letcher, A.J. and Harvey, P.H. 1994. Animal distributions: pattern and process. - In: Edwards, P.J., May, R.M. and Webb, N.R. (ed.), Largescale ecology and conservation biology. Blackwell Scientific Press, Oxford, pp. $41-58$.

Lei, G. and Hanski, I. 1997. Metapopulation structure of Cotesia melitaearum, a specialist parasitoid of the butterfly Meltiaea cinxia. - Oikos 78:91-100.

Levins, R. 1969. Some demographic and genetic consequences of environmental heterogeneity for biological control. - Bull. Ent. Soc. Amer. 15:237-240.

Nee, S. 1994. How populations persist. - Nature 367:123-124.

Nee, S. and May, R.M. 1992. Dynamics of metapopulations: habitat destruction and competitive coexistence. - J. Anim. Ecol. 61:37-40.

Nisbet, R.M. and Gurney, W.S.C. 1982. Modeling fluctuating populations. - John Wiley and Sons, New York.

Richards, S.A., Possingham, H.P. and Tizard, J. 1999. Optimal fire management for maintaining community diversity. - Ecol. Appl. 9:880:892.

Seneta, E. 1966. Quasi-stationary distributions and time-reversions in genetics. - J. Royal Statistical Soc., Series B 28:253-277.

Stone, L. 1995. Biodiversity and habitat destruction: a comparative study of model 
forest and coral reef systems. - Proc. Royal Soc. Lond. 262:381-388.

Tilman, D., May, R.M., Lehman, C.L. and Nowak, M.A. 1994. Habitat destruction and the extinction debt. - Nature 371:65-66.

With, K.A. and King, A.W. 1999. Extinction thresholds for species in fractal landscapes. - Cons. Biol. 13:314-326. 


\section{Chapter 4}

\section{A Markov chain model of a rocky subtidal community: succession and species interactions in a complex assemblage}

It is a magnificent feeling to recognize the unity of complex phenomena which appear to be things quite apart from the direct visible truth.

-Albert Einstein

\subsection{Introduction}

Marine hard substrate communities have proven to be ideal systems for studying the dynamics of multi-species assemblages. They are highly diverse, patchy communities that tend to be stable on large spatial scales but are relatively unstable on small spatial scales (Jackson 1977; Connell and Keough 1985; Sousa 1985). Experiments in these systems have shown the importance of a variety of biotic and abiotic processes, including competition (Connell 1961a,b, 1972; Paine 1974, 1976; Jackson 1977; Quinn 1982, Sebens 1986), predator-prey interactions (Menge and Sutherland 1976; Connell 1983, Witman 1985), mutualism (Vance 1978; Steneck 1982, Witman 1987), distur-

\footnotetext{
${ }^{1}$ This chapter been submitted to Ecology for publication.
} 
bance (Dayton 1971; Connell and Slatyer 1977; Paine and Levin 1981; Witman 1987; Jackson 1991; Hughes 1994), and recruitment (Sutherland 1974; Roughgarden et al. 1988; Gaines and Bertness 1992; Graham and Sebens 1996). Because these factors can interact in complex ways (e.g. direct species interactions can be nonlinear; shifts in species abundance can have indirect effects on the abundance of other species with which they do not interact), it is difficult to determine which ecological processes are most important for controlling community structure (Wootton 1993).

One method of untangling this complexity is to seek key processes or species whose loss would lead to large changes in the structure of the community (Bond 1994). A common approach to identifying these factors is species removal experiments (Paine 1974, 1992; Menge et al. 1994), in which the structure of a community is monitored in experimental plots following the removal of a species from the system. This approach has been successful in studies of intertidal communities where, for example, it has been shown that the exclusion of the starfish Pisaster ochraceus results in the competitive elimination of several sessile species by the mussel Mytilus californianus (Paine 1966, 1974). Species such as $P$. ochraceus whose removal produces a dramatic effect are termed strong reactors (Macarthur 1972; Paine 1980), or "keystone" species (Paine 1966). Unfortunately, in communities where no clear keystone species exists, it can be extremely difficult to quantify species interaction strengths and to characterize the relative importance of weak vs. strong interactions (Menge and Sutherland 1987; Paine 1992; Goldwasser and Roughgarden 1993; Laska and Wootton 1998). In many offshore marine habitats, such as the rocky subtidal zone, species removal experiments are impractical or difficult to perform. To characterize the processes governing the dynamics of such communities new theoretical approaches are required.

The combination of variability on small spatial scales and (relative) stability on larger spatial scales, typical of marine and hard-substrate systems, suggests the use of Markov chains as a description of community dynamics. These models have been used to characterize the dynamics of terrestrial forests (Waggoner and Stephens 1970; 
Horn 1975; Acevedo 1982; Runkle 1981; Masaki et al. 1992), plant communities (Isagi and Nakagoshi 1990; Aaviksoo 1995), and insect assemblages (Usher 1979). Their application in marine systems has been limited to a few studies on oceanic fisheries (Salia and Erxini 1987; Grant et. al. 1988; Formacion and Salia 1994) and a comprehensive analysis of a coral reef community (Tanneret al. 1994, 1996).

A Markov chain describes a community as a landscape of patches, each of which is in one of a number of possible states. The state of a patch is determined by the presence of an individual of a given species (or species group). The model is based on a transition matrix whose $(i, j)$ entry gives the probability that a patch in state $j$ changes to state $i$ in one time step. Markov chains usually converge to an equilibrium probability distribution of patch states, and most authors have focused on that distribution as a prediction of eventual community composition. There are, however, many other analytical tools available for the study of Markov chains that have not been widely used (Caswell and Cohen 1991a,b). In addition, sensitivity analyses originally developed for studying matrix population models (Caswell 1989) can be modified for use in Markov chains to investigate the effects of perturbations of the transition probabilities on model behavior.

In this paper we develop a simple Markov chain to characterize the dynamics of epifaunal invertebrate communities living on subtidal rock walls in the Gulf of Maine. Data for the model comes from permanent quadrats monitored over an 8 year period. We apply an array of analytical methods to the model to gain insights into the key processes underlying the observed structure of the community. These include a similarity analysis for classifying species into functional groups, several stochastic process indices (turnover rates, recurrence times, and first passage times) for quantifying successional dynamics at small spatial scales, and a set of sensitivity analysis for identifying the important factors and key species influencing diversity and community stability. While our focus here is on rocky subtidal communities, these methods are wholly applicable to any community of sessile organisms, such as plant 
communities, coral reefs, or rocky intertidal communities.

\subsection{Background: Rocky Subtidal Communities}

Much of our knowledge of marine hard substrate communities comes from research conducted in the rocky intertidal zone (e.g. Paine 1966; Menge 1976; Underwood and Denley 1984; Sousa 1985; Roughgarden et al. 1988). In comparison, the ecology of organisms living in rocky subtidal zone is much less well known (Witman and Dayton 2000). Because subtidal organisms inhabit regions that are typically subject to heavy fishing pressures and other human disturbances (Witman and Sebens 1992; Steneck 1997; Watling and Norse 1998) more attention needs to be focused on these regions if we hope to protect the integrity of subtidal systems.

The rocky subtidal zone encompasses the hard substrate habitat stretching from the intertidal fringe down to approximately 200 meters in depth (Witman and Dayton 2000). Communities are typically dominated by either algae or sessile invertebrates, which can occupy up to $90 \%$ of the available rock substrate at any one time (Sebens 1985; Witman and Dayton 2000). Diversity in subtidal communities is generally high, with many species coexisting on the substrate surface (Witman 1996). Factors thought to be important in maintaining subtidal diversity include predation (Ayling 1981; Duggins 1983; Witman and Cooper 1983), disease (Scheibling and Hennigar 1997) competition (Osman 1977, Sebens 1986), physical disturbance (Dayton et al 1970, Witman 1987, Witman and Dayton 2000), spatial heterogeneity (Witman 1985), recruitment (Smith and Witman 1999), sedimentation, current flow (Genovese 1996, Genovese and Witman 1999) and the richness of the biogeographical species pool (J.D.Witman, F. Smith and R.J. Etter, unpublished).

Space is frequently a limiting factor in rocky subtidal communities (Osman 1977; Russ 1982; Sebens 1986). Species typically compete for space by colonizing and holding on to empty space (Sebens 1986; Keough 1983), by eliminating nearby species 
through overgrowth competition (Russ 1982; Witman 1996), or by interference (Velimiron and Griffins 1979; Bruno and Witman 1996). In some subtidal communities overgrowth competition has been shown to be hierarchical with clear dominant species (Sebens 1986), while in others competition is best described as a network in which competitive interactions between species are often reciprocal (Buss and Jackson 1979; Russ 1982).

The slope of the rock surface and depth can also affect subtidal community structure. At shallow depths, horizontal and gently sloping substrates are generally dominated by macroalgae, while vertical rock walls are dominated by epifaunal invertebrates (Witman and Cooper 1983; Sebens 1985; 1986, Witman and Sebens 1988; Bruno and Witman 1996; Witman and Grange 1998). This pattern is probably regulated by multiple factors but is undoubtedly related to higher light levels on horizontal substrates, which creates a more favorable environment for the growth and survival of macroalgae (Witman and Cooper 1983). With increasing depth, the abundance of sessile invertebrates increases and the abundance of macroalgae decreases (Vadas and Stenck 1988; Witman and Sebens 1988). Thus differences between horizontal and vertical rock wall communities are less distinct at depths greater than 30 meters (Witman and Dayton 2000).

The focus of our study is a vertical rock wall community located at approximately 30 meters depth on Ammen Rock.Pinnacle in the Gulf of Maine (Witman and Sebens 1988; Leichter and Witman 1997). The data for our model was collected over an eight-year period (1986-1994). It consists of a series of photographs chronicling the spatial distribution of sessile species on the rock wall substrate through time. Ten replicate quadrats, positioned randomly along a 20 meter span of rock wall habitat were photographed at least yearly with a Nikonos $V$ camera mounted on a quadrapod frame (as in Witman 1985). Color prints were made of the high resolution color slides to identify the species of five major taxa of epifaunal invertebrates (sponges, sea anemones, ascidians, bryozoans, and polychaetes) and a species of coralline algae 


\begin{tabular}{lll}
\hline \hline Model States & Species Type & State ID \\
\hline Bare Rock & & BR \\
Hymedesmia 1 sp. & Sponge & HY 1 \\
Hymedesmia 2 sp. & Sponge & HY 2 \\
Myxilla fimbriata & Sponge & MYX \\
Mycale lingua & Sponge & MYC \\
Metridium senile & Sea anemone & MET \\
Urticina crassicornis & Sea anemone & URT \\
Aplidium pallidum & Ascidian & APL \\
Ascidia callosa & Ascidian & ASC \\
Parasmittina jeffreysi & Bryozoan & PAR \\
Idmidronea atlantica & Bryozoan & IDM \\
Crisia eburnea & Bryozoan & CRI \\
Filograna implexa & Polychaete & FIL \\
Spirorbis spirorbis & Polychaete & SPI \\
Coralline Algae & Encrusting algae & COR \\
\hline \hline
\end{tabular}

Table 4.1: Subtidal species identified in ten quadrats located at 30 meters depth on Ammen Rock pinnacle in the Gulf of Maine. Species are identified in the model using the state codes in the right-hand column of the table.

(Fig. 4.1). A total of 14 species were identified in the quadrats (Table 4.1).

\subsection{Model Structure and Analysis}

We modeled the dynamics of the subtidal community using a Markov chain. The model is defined by its transition matrix $\mathbf{A}$, whose elements, $a_{i j}$, give the probability that a patch (i.e. a small discrete area on the rock substrate) in state $j$ at time $t$ changes to state $i$ at time $t+1$. The matrix $\mathbf{A}$ is nonnegative (all $a_{i j} \geq 0$ ) and has the property that each column sums to 1 (i.e. $\mathbf{A}$ is column-stochastic).

The state of a patch is defined by the species that occupies it. A patch can also be empty (bare rock). Thus, since there are 14 species in our data set, the number of states in our model is 15 . Patch states are identified by the abbreviations given in Table 4.1.

In the model, the dynamics of the community are described in terms of patch tran- 


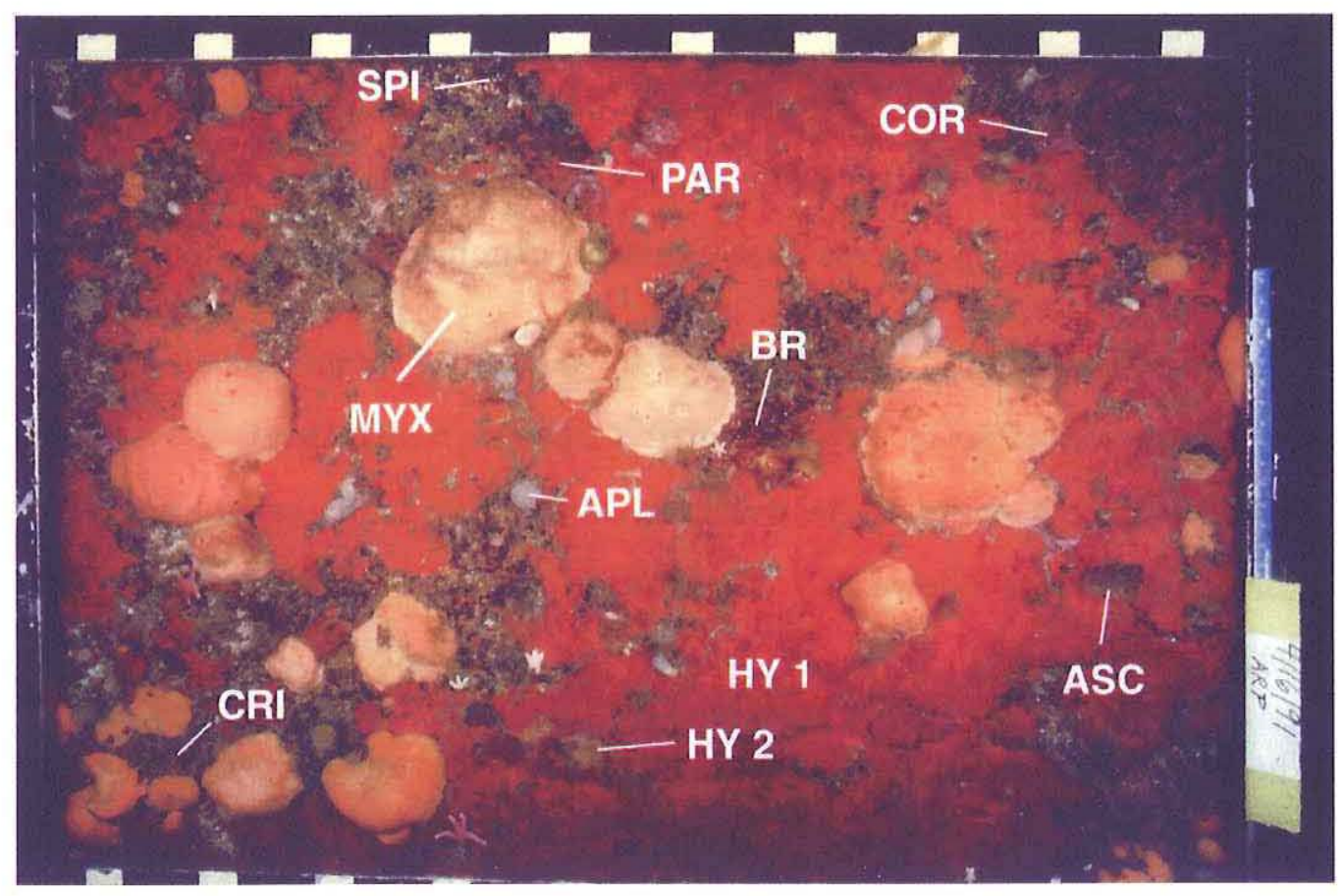

Figure 4.1: Photo quadrat showing most of the 14 species of epifaunal invertebrates investigated. The thinly encrusting sponge Hymedesmia species 1 (HY1) dominates most of the space in this particular quadrat. The orange mounding sponge Myxilla fimbriata (MYX) is also prominent. Species abbreviations are as in Table 4.1. Not shown are the sea anemones, Urticina crassicornis and Metridium senile, which occur in large aggregations, the polychaete Filograna implexa, and the bryozoan, Idmidronea atlantica. 
sition probabilities. Transition probabilities depend on several ecological processes affecting species abundances.

1. The probability that an empty patch becomes occupied is a function of colonization. Colonization of a patch occurs either by successful recruitment of larvae onto bare substrate or by growth of individuals into unoccupied space.

2. The probability that an occupied patch becomes empty is a function of disturbance. Disturbance within a patch is a result of predation, physical disturbance, disease, or any other process that cause an occupied patch to become empty.

3. The probability that a patch occupied by species $j$ becomes occupied by species $i$ is a function of species replacement. Species replacement can occur either directly through competitive overgrowth (Sebens 1985) or indirectly when a mortality event is followed by colonization within a single time step (Witman 1987, 1996).

4. The probability a that patch occupied by species $i$ remains occupied by species $i$ is a function of persistence. Species that are resistant to disturbance and competitive replacement have high persistence probabilities.

The proportion of patches in each state gives a description of the species composition of the community. Let $\mathbf{x}(\mathrm{t})$ be a column vector giving the probability distribution of patch states at time $t$. Then the species composition at time $t+1$ is given by

$$
\mathbf{x}(t+1)=\mathbf{A} \mathbf{x}(t)
$$

In our model the time interval is one year. This interval was chosen because subannual observations of quadrat photos show minimal variation in species composition. Thus multiplication of $\mathbf{x}(\mathrm{t})$ by $\mathrm{A}$ projects the community vector forward one year in time. 


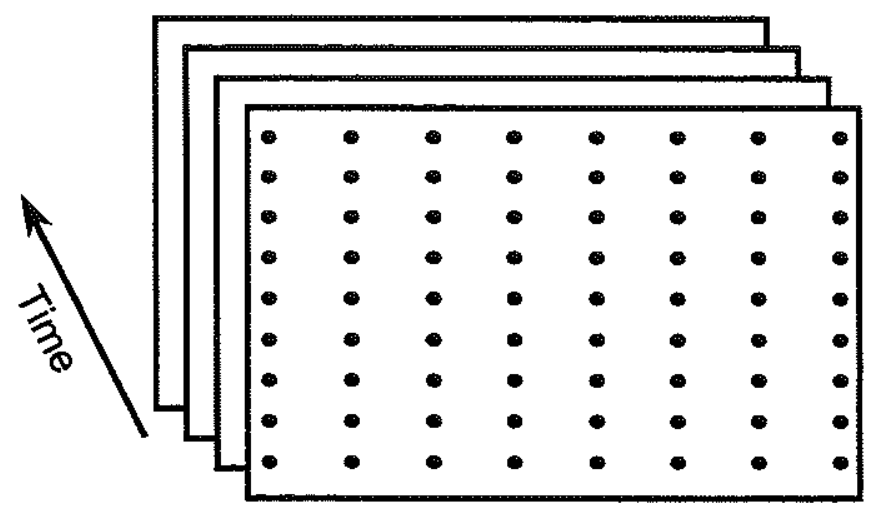

Figure 4.2: Cartoon showing the method used to collect state transition data from quadrat photos. The squares represent photos from a single quadrat taken at yearly intervals. A lattice of approximately 600 points was placed on each quadrat photo and species transitions under each point were followed through time. Points were space approximately $1 \mathrm{~cm}$ apart and are considered to represent a $1 \mathrm{~cm}^{2}$ patch on the substrate wall.

The largest eigenvalue of $\mathbf{A}$ equals 1. The corresponding eigenvector, $\mathbf{w}$, gives the equilibrium distribution of patch states. If $\mathbf{A}$ is primitive, the community will asymptotically converge (as $t \rightarrow \infty$ ) to $\mathbf{w}$ from any initial condition. The $i$ th element of $\mathbf{w}\left(w_{i}\right)$ gives the proportion of the landscape occupied by species $i$ at equilibrium.

\subsubsection{Parameter estimation}

Data to construct the transition matrix were obtained by superimposing a lattice of evenly spaced points over the quadrat color prints $(30 \times 20 \mathrm{~cm})$ and counting patch transitions through time (Fig. 4.2). Approximately 600 points (a single point every $1 \mathrm{~cm}$ ) were assayed per quadrat. We chose this scale because it was approximately equivalent to the size of the smallest organism in our data set. For simplicity we will refer to each point as a patch, where the size of a patch is taken to be $1 \mathrm{~cm}^{2}$. Since individuals of many of the subtidal species are capable of growing much larger than $1 \mathrm{~cm}^{2}$, a single individual can occupy more than one patch. 
The transition probabilities were estimated by constructing a two way contingency table in which the $i, j$ th entry gives the number of patches that were in state $j$ at some time $t$ and in state $i$ the following year. We constructed the contingency table by pooling the data among all quadrats and all time intervals. The transition probabilities, $a_{i j}$, were calculated as

$$
a_{i j}=\frac{n_{i j}}{n_{j}}
$$

where $n_{i j}$ is the number of transitions from state $j$ to state $i$ (the $i, j$ th entry of the contingency table), and $n_{j}$ is the total number of transitions starting in state $j$ at time $t$ (sum of the $j$ th column of the contingency table).

By pooling the data, we are averaging over small scale spatial variability and small scale temporal variability to produce the best single realization of a Markov chain for the subtidal community. The questions we are exploring with our model concern the expected behavior of the community, given that the present conditions are maintained indefinitely. As we will show, analyses of homogenous Markov chains can reveal important information about the processes giving rise to observed patterns of species abundances. The effects of temporal and spatial variability on model behavior are explored in a separate paper (Hill et al. 2000 in prep).

\subsubsection{Identifying Functional Groups}

The number of states in our model is large. To make the Markov chain more tractable, we introduce a method for combining species into groups based on the functional similarity of species roles within the community. Typically, species are combined into functional groups based on their degree of taxonomic relatedness (e.g., Waggoner and Stephens 1970, Saila and Erzini 1987, Tanner et al. 1994). The problem with this approach, however, is that taxonomically related species may have functionally different effects on community dynamics. 
To form functional groups we used a compression algorithm for Markov chain matrices developed by Spears (1998). The algorithm works by combining a pair of states $i$ and $j$ together to create a new state $i \cup j$ that represents the probability of being in either state $i$ or state $j$. Spears shows that the probability of a transition from any state $k$ into state $i \cup j$ is simply the sum of the individual state probabilities.

$$
a_{i \cup j, k}=a_{i, k}+a_{j, k}
$$

while the probability of a transition from state $i \cup j$ to any state $k$ is a weighted average of the elements $a_{k i}$ and $a_{k j}$,

$$
a_{k, i \cup j}=\frac{m_{i} a_{k, i}+m_{j} a_{k, j}}{m_{i}+m_{j}}
$$

where $m_{i}$ is the sum of all the elements in the $i$ th row of $\mathbf{A}$.

The functional similarity among species is determined by measuring the distance between the rows and columns of $\mathbf{A}$. The distance between the rows and columns associated with states $i$ and $j$ is given by

$$
\delta_{i j}=\left(\sum_{k}\left|\frac{m_{i} a_{j, k}-m_{j} a_{i, k}}{m_{i}+m_{j}}\right|\right)\left(\sum_{l}\left|a_{l, i}-a_{l, j}\right|\right)
$$

(Spears 1998). The value of $\delta_{i j}$ can be thought of as a measure of the degree of functional dissimilarity between states $i$ and $j$. Pairs of species with low values of $\delta_{i j}$ are good candidates for combing into functional groups.

Combining a pair of states reduces the dimension of the transition matrix by one. The algorithm for compressing the transition matrix is as follows:

1. Calculate the distance $\delta_{i j}, i, j=1, \ldots, S$, between all pairs of states.

2. Find the pair of states $i^{*}$ and $j^{*}$ for which $\delta_{i^{*} j^{*}}=\min _{i, j}\left(\delta_{i j}\right)$.

3. Compute a weighted average of columns $i^{*}$ and $j^{*}$ (using Eq. 4.5)and place the 
result in columns $i^{*}$ and $j^{*}$.

4. Add rows $i^{*}$ and $j^{*}$ and place the result in row $i^{*}$.

5. Delete column $j^{*}$ and row $j^{*}$ from the matrix.

This process can be iterated by applying the algorithm to the new matrix $\mathbf{A}_{i^{*} \cup j^{*}}$ (where $\mathbf{A}_{i^{*} \cup j^{*}}$ is the transition matrix for the combined state $i^{*} \cup j^{*}$ ).

Combing states $i^{*}$ and $j^{*}$ results in the loss of some information from the model. We can quantify the loss of information by calculating the percent change $(\Phi)$ in the equilibrium distribution as

$$
\Phi=\frac{\left|\mathbf{w}_{i \cup j}-\mathbf{w}^{\prime}\right|}{2} \times 100
$$

where $\mathbf{w}_{i^{*} \cup j^{*}}$ is the dominant eigenvector of $\mathbf{A}_{i^{*} \cup j^{*}}$, and $\mathbf{w}^{\prime}$ is a vector formed by setting element $i^{*}$ of $\mathbf{w}$ equal to the sum of elements $i^{*}$ and $j^{*}$ and then deleting element $j^{*}$.

\subsubsection{Rates and patterns of succession}

A Markov chain describes two spatial scales-the regional dynamics of the community and the local dynamics of patches. Although the community converges to an equilibrium distribution of patches (characterized by $\mathbf{w}$ ), individual patches change state continuously through time.

To investigate rates of successional change at the spatial scale of a patch, we calculated mean turnover rates, Smouchowski recurrence times for each state, and the mean first passage times for pairs of states. These indices are a well-known part of the literature on Markov chains (e.g., Iosifescu 1980); they provide insights into rates of species change within patches and patterns of succession (Caswell and Cohen 1991a,b). 
- The mean turnover rate describes the probability that a randomly selected patch changes state between $t$ and $t+1$, and is given by

$$
\sum_{i=1}^{s} w_{i}\left(1-a_{i i}\right)
$$

where $w_{i}$ is the $i$ th element of the dominant eigenvector, and $\left(1-a_{i i}\right)$ is the probability that a patch in state $i$ changes states in the time interval from $t$ to $t+1$ (Caswell and Cohen 1991b).

- The Smouchowski recurrence time $\theta_{i}$ of state $i$ is the time elapsing between a patch leaving state $i$ and then returning to it again. Its mean is given by

$$
\theta_{i}=\frac{1-w_{i}}{w_{i}\left(1-a_{i i}\right)}
$$

(Iosifescu 1980). A particularly informative measure is the Smouchowski recurrence time for bare rock $\left(\theta_{1}\right)$. Its value gives the mean time a patch stays occupied once it has been colonized.

- The mean first passage time is the average time it takes for a patch in state $j$ to first reach state $i$. This measure can provide insights into the relative rates of succession. Let $\tau_{i j}$ denote the mean first passage time from state $j$ to state $i$. The matrix $\mathbf{T}$ whose elements are $\tau_{i j}$ is given by

$$
\mathbf{T}=\left(\mathbf{V}_{\mathbf{d g}}\right)^{-1}\left(\mathbf{I}-\mathbf{Z}+\mathbf{Z}_{\mathrm{dg}} \mathbf{E}\right)
$$

where $\mathbf{V}$ is a matrix whose columns all equal $\mathbf{w}, \mathbf{Z}$ is a matrix given by $\mathbf{Z}=[\mathbf{I}-(\mathbf{A}-\mathbf{V})]^{-1}$ (I is the identify matrix), $\mathbf{E}$ is a matrix of ones, and the subscript $d g$ denotes matrices containing the diagonal elements of $\mathbf{V}$ and $\mathbf{Z}$ (Iosifescu 1980). Note that the mean recurrence time for a state is given by the diagonal elements $\tau_{i i}$ of $\mathbf{T}$. The difference between $\tau_{i i}$ and $\theta_{i}$, however, is that 
$\tau_{i i}$ can be heavily influenced by patches remaining in state $i$ from time $t$ to $t+1$ (Caswell and Cohen 1991b). Thus, $\theta_{i}$ is a better estimate of recurrence times than $\tau_{i i}$.

\subsubsection{Sensitivity analysis}

We used sensitivity analysis to investigate how species abundance would vary in response to changes in the elements of $\mathbf{A}$. The effect of such changes provides a measure of how important a given transition probability is to the overall composition of the community. To quantify this effect, we derive sensitivity formulas for the dominant eigenvector of a Markov chain and for a scalar measure of species diversity.

Eigenvector sensitivities provide information on how the equilibrium distribution of patches $\mathbf{w}$ changes in response to changes in A. Tanner et al. (1984) attempted such an analysis for a Markov chain of a coral reef community, but their calculations are flawed because they failed to account for the fact that the column sums of a Markov chain transition matrix must sum to one.

The dominant right eigenvector (here represented as $\mathbf{w}_{1}$ ) of $\mathbf{A}$ gives the stationary community structure distribution. The sensitivity of each element in $\mathbf{w}_{1}$ to changes in A can be found using Caswell's $(1989,2000)$ formula for scaled eigenvectors

$$
\frac{d \frac{\mathbf{w}_{1}}{\left\|\mathbf{w}_{1}\right\|}}{d a_{i j}}=\frac{\partial \frac{\mathbf{w}_{1}}{\left\|\mathbf{w}_{1}\right\|}}{\partial a_{i j}}+\sum_{m \neq i}^{s} \frac{\partial \frac{\mathbf{w}_{1}}{\left\|\mathbf{w}_{1}\right\|}}{\partial a_{m j}} \frac{\partial a_{m j}}{\partial a_{i j}}
$$

If the dominant eigenvector is already scaled so $\left\|\mathbf{w}_{1}\right\|=1$, then

$$
\frac{\partial \frac{\mathbf{w}_{1}}{\left\|\mathbf{w}_{1}\right\|}}{\partial a_{i j}}=\frac{\partial \mathbf{w}_{1}}{\partial a_{i j}}-\mathbf{w}_{1} \sum_{k} \frac{\partial w_{k}}{\partial a_{i j}}
$$

where

$$
\frac{\partial \mathbf{w}_{1}}{\partial a_{i j}}=w_{j}^{(1)} \sum_{m \neq 1}^{s} \frac{v_{i}^{(m)}}{\lambda_{1}-\lambda_{m}} \mathbf{w}_{m}
$$

$w_{j}^{(1)}$ is the $j$ th element of $\mathbf{w}_{1}, v_{i}^{(m)}$ is element $i$ of the left eigenvector $\mathbf{v}_{m}$, and $\lambda_{m}$ is 
the mth eigenvalue.

The derivatives $\partial a_{k j} / \partial a_{i j}$ in (4.10) are determined so that the change in $a_{i j}$ is compensated by the other entries in column $j$ of $\mathbf{A}$. While many compensation patterns are possible (Caswell 2000), here we used proportional compensation

$$
\frac{\partial a_{k j}}{\partial a_{i j}}=\frac{-a_{k j}}{1-a_{i j}}
$$

in which the change in $a_{i j}$ is distributed over the other the entries in the $j$ th column proportional to their value.

Eigenvector sensitivities provide insights into how changes in transition probabilities affect community structure; however, they can also be cumbersome to interpret. A simpler approach, akin to the concept of eigenvalue sensitivities in demographic modeling, is to use equation (4.10) to compute the sensitivity of a summary statistic describing community structure.

A summary statistic commonly used in community ecology is the Shannon-Wiener diversity index $(H)$. Using this index, the diversity of the stationary distribution can be calculated as

$$
H=-\sum_{i} w_{i} \ln w_{i}
$$

The sensitivity of $H$ to changes in $a_{i j}$ is derived by taking the derivative of $H$ with respect to $a_{i j}$

$$
\frac{\partial H}{\partial a_{i j}}=-\sum_{k}\left(\ln w_{k}+1\right) \frac{d w_{k}}{d a_{i j}}
$$

where $d w_{k} / d a_{i j}$ is the $k$ th element of $d \mathbf{w} / d a_{i j}$. Equation 4.15 provides a way of characterizing how changes in the transition probabilities affect a scalar measure of community diversity. 


\subsubsection{Effects of species removals}

We characterized the functional importance of each species by calculating the effect of its removal on community diversity. Diversity, as defined by the Shannon-Wiener index $H$, is a measure of both species richness and the evenness in species abundance (Lloyd and Ghelardi 1964). Because species removal automatically affects richness, we want a measure that is sensitive only to the evenness component of diversity. Evenness $J(S)$ for a community with $S$ species is defined by the ratio

$$
J(S)=\frac{H}{\ln (S)}
$$

where $0 \leq J(S) \leq 1$.

To simulate the removal of species $i$, we first set row $i$ and column $i$ of $\mathbf{A}$ to zero and renormalized all the other columns so they sum to one. Call this matrix, with species $i$ removed, $\mathbf{A}_{i}$ and its equilibrium distribution $\mathbf{w}^{(i)}$. Then the percent change in evenness $\Delta J_{i}$ is

$$
\Delta J_{i}=\frac{J_{i}(S-1)-J(S)}{J(S)} \times 100
$$

where $J_{i}(S-1)=H_{i} / \ln (S-1)$ and $H_{i}$ is obtained from $\mathbf{w}^{(i)}$. If the removal of species $i$ has no effect on the relative abundance of the rest of the community, $\Delta J_{i}=0$. If $\Delta J_{i}<0$, then species $i$ has a positive effect on evenness. If $\Delta J_{i}>0$ then the presence of species $i$ has a negative effect on diversity.

Species removal also affects community resilience (i.e. the rate the community converges to equilibrium). We measured resilience using Dobrushin's coefficient of ergodicity

$$
\bar{\alpha}(\mathbf{A})=\frac{1}{2} \max _{j, k} \sum_{i=1}^{S}\left|a_{i j}-a_{i k}\right|
$$

(Dobrushin 1956a,b). The coefficient $\bar{\alpha}(\mathbf{A})$ satisfies the condition

$$
\bar{\alpha}(\mathbf{A})=\sup _{\mathbf{x}_{1} \mathbf{x}_{2}}\left\{\frac{\left\|\mathbf{A} \mathbf{x}_{1}-\mathbf{A} \mathbf{x}_{2}\right\|}{\left\|\mathbf{x}_{1}-\mathbf{x}_{2}\right\|}\right\}
$$


where $\mathbf{x}_{1}$ and $\mathbf{x}_{2}$ are probability vectors of size $S \times 1$ whose elements sum to 1 $\left(\mathbf{x}_{1} \neq \mathrm{x}_{2}\right)$. The value of $\bar{\alpha}(\mathbf{A})$ gives the minimum contraction in the distance between two initial vectors after multiplication by $\mathbf{A}$. Thus the lower the value of $\bar{\alpha}(\mathbf{A})$, the faster the community is predicted to converge to equilibrium.

Dobrushin's coefficient $\bar{\alpha}(\mathbf{A})$ gives the minimum contraction rate, but does not show how that minimum relates to the typical contraction rate. To quantify this typical rate, we calculated the distribution for the contraction rate

$$
\phi=\frac{\left\|\mathbf{A} \mathbf{x}_{1}-\mathbf{A} \mathbf{x}_{2}\right\|}{\left\|\mathbf{x}_{1}-\mathbf{x}_{2}\right\|}
$$

by randomly selecting 10000 pairs of probability vectors, $\mathbf{x}_{1}$ and $\mathbf{x}_{2}$, from a uniform distribution on the $S-1$ simplex. We represent the mean of this distribution as $\bar{\phi}$.

If the removal of species $i$ increases $\bar{\alpha}(\mathbf{A})$ or $\bar{\phi}$ then species $i$ has a stabilizing affect on the community. If the removal of species $i$ decreases $\bar{\alpha}(\mathbf{A})$ or $\bar{\phi}$ then species $i$ has a destabilizing affect on the community.

The measures $\bar{\alpha}(\mathbf{A})$ and $\bar{\phi}$ give measures of community resilience by estimating contraction ratios starting from any initial condition. We chose these measures because disturbances in ecological systems can be quite large (Dayton 1971; Witman 1987).

\subsection{Results}

The transition matrix $\mathbf{A}$ is shown in Table 4.2. The matrix is organized so that colonization probabilities are given in the first column (Bare Rock $\rightarrow$ Species $i$ ) and disturbance probabilities are given in the first row (Species $i \rightarrow$ Bare Rock). Persistence probabilities are located along the diagonal of the matrix (bold elements), while species replacement probabilities are given by the off diagonal elements of $\mathbf{A}$ (excluding the first row and column).

The first column of $\mathbf{A}$ shows that colonization probabilities are highly variable, 


\begin{tabular}{|c|c|c|c|c|c|c|c|c|c|c|c|c|c|c|c|}
\hline \multicolumn{16}{|c|}{ From } \\
\hline & $\mathrm{BR}$ & HY 1 & HY2 & MXY & $\mathrm{MYC}$ & MET & URT & APL & ASC & PAR & IDM & CRI & FIL & SPI & $\mathrm{COR}$ \\
\hline To:BR & 0.294 & 0.029 & 0.074 & 0.084 & 0.036 & 0.266 & 0.036 & 0.122 & 0,116 & 0.076 & 0.104 & 0.069 & 0.108 & 0.127 & 0.076 \\
\hline HY 1 & 0.101 & 0.771 & 0.056 & 0.052 & 0.017 & 0.026 & 0.009 & 0.199 & 0.242 & 0.321 & 0.309 & 0.145 & 0.117 & 0.158 & 0.056 \\
\hline HY 2 & 0.013 & 0.002 & 0.586 & 0.025 & 0.008 & 0.004 & 0.016 & 0.015 & 0.014 & 0.003 & 0.010 & 0.011 & 0.013 & 0.018 & 0.007 \\
\hline MYX & 0.025 & 0.017 & 0.026 & 0.710 & 0.006 & 0.018 & 0.012 & 0.038 & 0.051 & 0.023 & 0.031 & 0.031 & 0.035 & 0.018 & 0.028 \\
\hline MYC & 0.010 & 0.004 & 0.011 & 0.004 & 0.839 & 0.030 & 0.000 & 0.018 & 0.016 & 0.000 & 0.010 & 0.011 & 0.004 & 0.018 & 0.008 \\
\hline MET & 0.017 & 0.002 & 0.005 & 0.007 & 0.011 & 0.537 & 0.008 & 0.020 & 0.005 & 0.000 & 0.008 & 0.008 & 0.005 & 0.006 & 0.002 \\
\hline URT & 0.005 & 0.001 & 0.006 & 0.004 & 0.001 & 0.000 & 0.863 & 0.007 & 0.024 & 0.000 & 0.006 & 0.005 & 0.008 & 0.006 & 0.000 \\
\hline APL & 0.034 & 0.012 & 0.026 & 0.008 & 0.006 & 0.016 & 0.008 & 0.154 & 0.041 & 0.020 & 0.031 & 0.025 & 0.032 & 0.018 & 0.020 \\
\hline ASC & 0.048 & 0.019 & 0.011 & 0.008 & 0.004 & 0.010 & 0.002 & 0.044 & 0.105 & 0.030 & 0.042 & 0.022 & 0.033 & 0.030 & 0.025 \\
\hline PAR & 0.011 & 0.005 & 0.001 & 0.002 & 0.000 & 0.003 & 0.000 & 0.009 & 0.014 & 0.248 & 0.012 & 0.005 & 0.006 & 0.000 & 0.005 \\
\hline $\mathrm{IDM}$ & 0.031 & 0.014 & 0.021 & 0.004 & 0.004 & 0.003 & 0.003 & 0.027 & 0.033 & 0.020 & 0.165 & 0.015 & 0.007 & 0.030 & 0.007 \\
\hline CRI & 0.320 & 0.102 & 0.147 & 0.061 & 0.054 & 0.068 & 0.024 & 0.235 & 0.223 & 0.179 & 0.228 & 0.609 & 0.218 & 0.449 & 0.222 \\
\hline FIL & 0.062 & 0.016 & 0.020 & 0.020 & 0.005 & 0.016 & 0.016 & 0.089 & 0.080 & 0.063 & 0.027 & 0.028 & 0.404 & 0.085 & 0.036 \\
\hline SPI & 0.013 & 0.003 & 0.005 & 0.008 & 0.003 & 0.003 & 0.000 & 0.009 & 0.012 & 0.000 & 0.006 & 0.004 & 0.005 & 0.030 & 0.003 \\
\hline $\mathrm{COR}$ & 0.017 & 0.003 & 0.006 & 0.005 & 0.007 & 0.001 & 0.005 & 0.016 & 0.025 & 0.017 & 0.013 & 0.012 & 0.006 & 0.006 & 0.507 \\
\hline
\end{tabular}

Table 4.2: Transition matrix for the subtidal community. The $(i, j)$ element in the matrix is the probability that a patch in state $j$ at time $t$ changes to state $i$ at time $t+1$. The transition time $(t, t+1)$ is one year. State codes are given in table 4.1 . 
ranging over almost two orders of magnitude. The best colonizer by far is the bryozoan Crisia $\left(a_{1,12}=0.32\right)$, which is three times as likely as the next best colonizer (Hymedesmia $1 ; a_{1,2}=0.10$ ) to occupy a previously empty patch. The remaining species in the community have much lower colonization rates (probabilities range between 0.005 and 0.062 ). The probability that an empty patch will not be colonized by any species over a one-year period is 0.29 .

Persistence probabilities and disturbance probabilities also vary a great deal among species, reflecting a wide range of variation in invertebrate life histories. The species with the largest persistence probabilities are Urticina (0.863) and Mycale (0.839), while the species with the smallest persistence probability is Spirorbis (0.03). The species with the largest disturbance probability is Metridium (0.266), while the species with the smallest disturbance probability is Hymedesmia 1 (0.029).

Approximately $94 \%$ of the replacement probabilities (off-diagonal elements of A) and $90 \%$ of the symmetric pairs of replacement probabilities are non-zero $\left(a_{i j}\right.$ and $a_{j i}>0 ; i, j \neq 1$ ), indicating that competition between species is reciprocal (i.e. species $i$ can replace species $j$, and species $j$ can replace species $i$ ). Most replacement transitions, however, are improbable, with $84 \%$ of them having probabilities less than 0.05. Replacement probabilities greater than 0.05 are generally much larger than their symmetrical pair $\left(a_{i j}>>a_{j i}\right.$ or visa versa), indicating that species competition is directional (i.e. species $i$ replaces species $j$ more often than $j$ replaces $i$ ). The one exception is for the species Hymedesmia 1 and Crisia, which tend to replace each other with approximately equal probability.

The row sum $\left(R S=\sum_{\substack{j \neq 1 \\ j \neq i}} a_{i j}\right)$ of the elements of the ith row of A gives a measure of the competitive ranking of species $i$ relative to other species in the community. The highest ranked competitors are Crisia $(R S=2.21)$, Hymedesmia $1(R S=1.71)$ and Filograna $(R S=0.50)$. The lowest ranked competitors are Parasmittina $(R S=$ $0.061)$, Spirorbis $(R S=0.062)$, and Urticina $(R S=0.067)$. 
a.

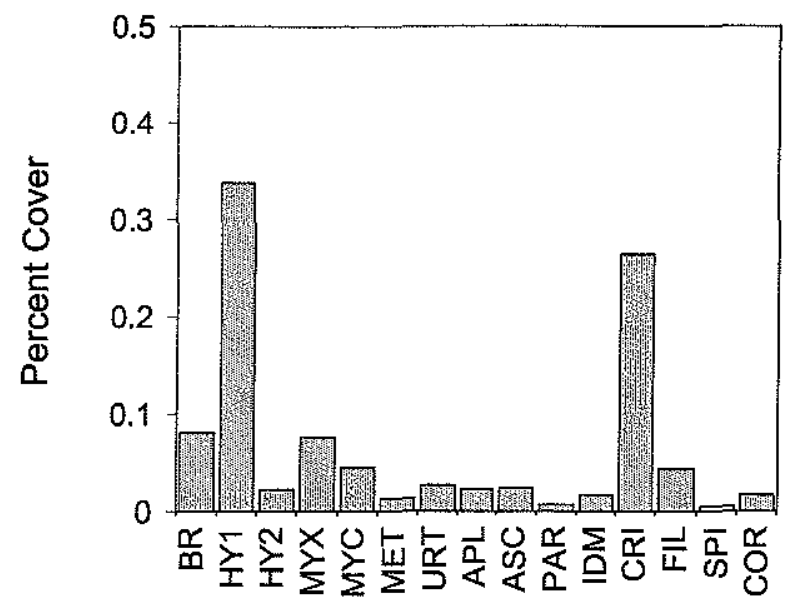

b.

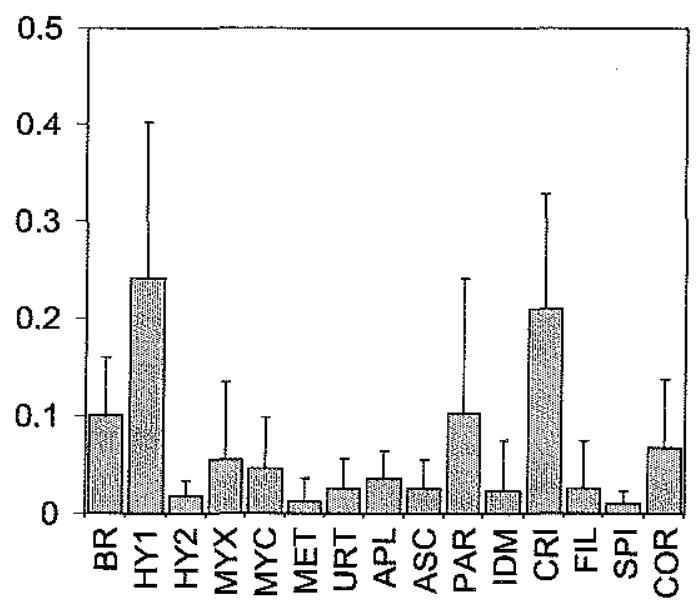

Figure 4.3: Predicted and observed species abundances. a. Predicted equilibrium abundances of subtidal species (given as the percent cover of substrate) at Ammen rock in the Gulf of Maine, as given by the dominate eigenvector $\mathbf{w}$ of the transition matrix A. $b$. Observed species abundances at three rock wall sites located nearby the quadrats for the years 1987, 1990, and 1992. The observed values are means taken over all 3 sites and across all 3 years. Error bars represent 1 S.D.

\subsubsection{Equilibrium distribution}

The equilibrium distribution of patch states is shown in Figure 4.3a. At equilibrium the model predicts that sponges (Hymedesmia spp., Myxilla, and Mycale) will occupy over $50 \%$ of the rock substrate, and that Hymedesmia 1 and the bryozoan Crisia will dominate the community (together occupying $58 \%$ of the substrate). As a comparison, Figure $4.3 \mathrm{~b}$ shows the mean relative abundance distribution at six quadrats located within 75 meters of the model quadrats. Mean abundances are for the years 1987, 1990, and 1991, and include only those species represented by our model (about $20 \%$ of the substrate was occupied by species not found in the model quadrats). The equilibrium abundances predicted by the Markov chain fall within the range of species variability observed among the six independent quadrats. 


\subsubsection{Quantifying functional relatedness and combining species into functional groups}

To quantify the degree of relatedness between species in the subtidal community, we generated a dissimilarity dendogram based on Spears' distance metric $\delta_{i j}$ (Fig. 4.4). The dendogram was constructed using the UPGMA method (unweighted pair group method with arithmetic mean) (Ayala 1976). The most similar states are bare rock and the polychaete Spirorbis $(\delta=0.022)$. This implies that the other species do not perceive a patch occupied by Spirorbis differently than an unoccupied patch. The next most similar states are the ascidians, Aplidium and Ascidia $(\delta=0.040)$, followed by the pairing of the ascidians with the bryozoan Idmidronea $(\delta=0.081)$. The dendogram shows there is much more functional redundancy among the bryozoans, ascidians, and polychaetes, then among the sponges and sea anemones, and that species do not necessarily cluster according to taxonomic groups.

Figure 4.5 shows the percent change, $\Phi$, in the equilibrium distribution (Eq. 4.6) as a function of the reduction in the number of states in the model. We reduced the size of the state space by combining pairs of states in order of their similarity $\left(\delta_{i j}\right)$, using the compression algorithm described in section (4.3.2). As more states are combined $\Phi$ increases, rising to a maximum of about $20 \%$ after combing 13 out of the original 15 states (at which point $\mathbf{A}$ is a $2 \times 2$ matrix).

We set a threshold level of $\Phi=1 \%$ (dashed line in Fig. 4.5) as a cutoff criteria for combining species into functional groups. Reducing the number of states by five produces a change in the equilibrium distribution of about $0.7 \%$. The order in which these states are combined by the compression algorithm is as follows:

- BR with SPI

- APL with ASC

- $[\mathrm{APL} \cup \mathrm{ASC}]$ with $\mathrm{IDM}$ 


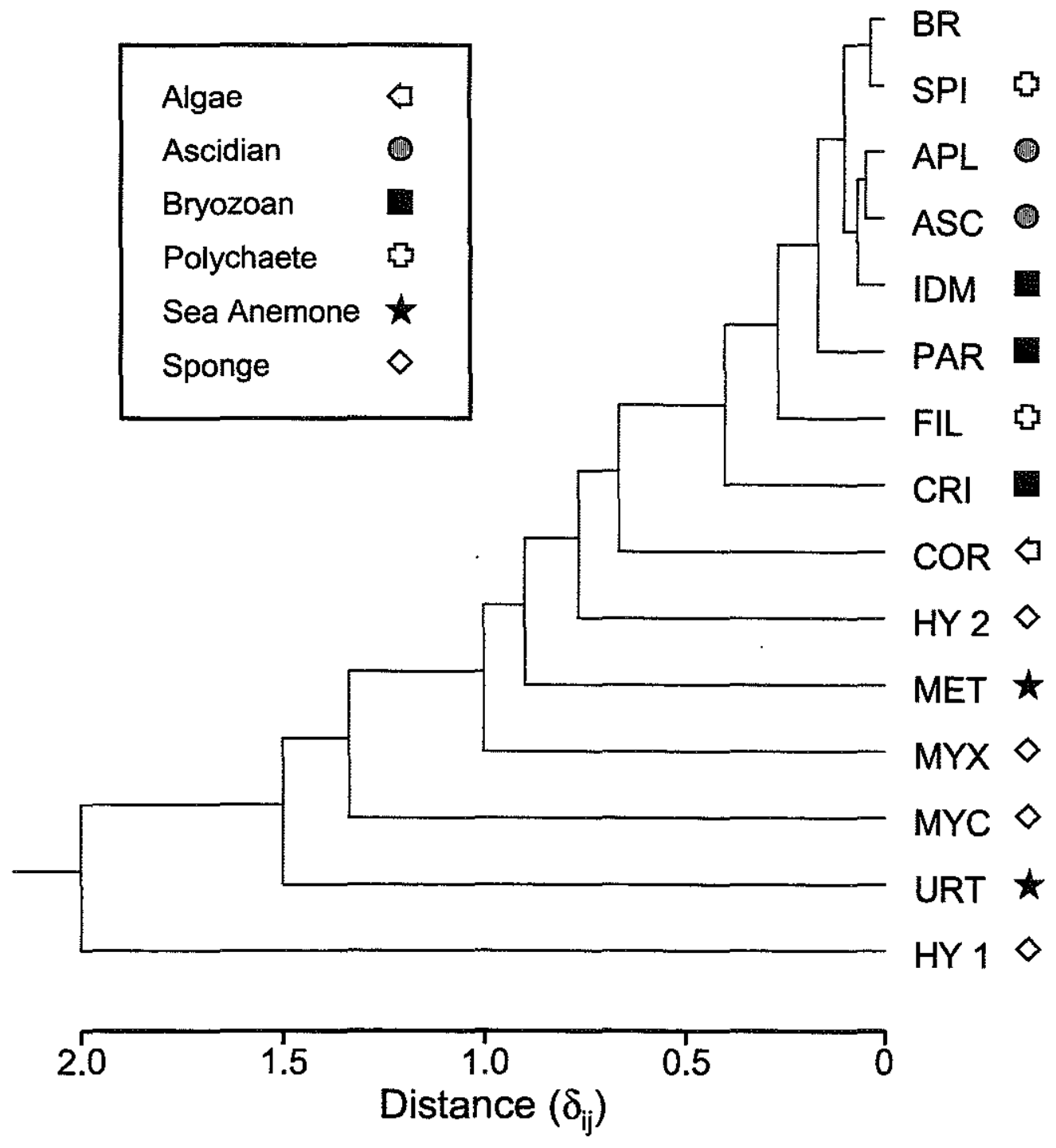

Figure 4.4: Distance dendogram of species states generated using Spears' $\delta_{i j}$ metric. States are clustered according to their degree of functional similarity with other states measured as the distance between the rows and columns of the transition matrix A (see text for details). The dendogram was created using the UPGMA method (unweighted pair group method with arithmetic mean). 


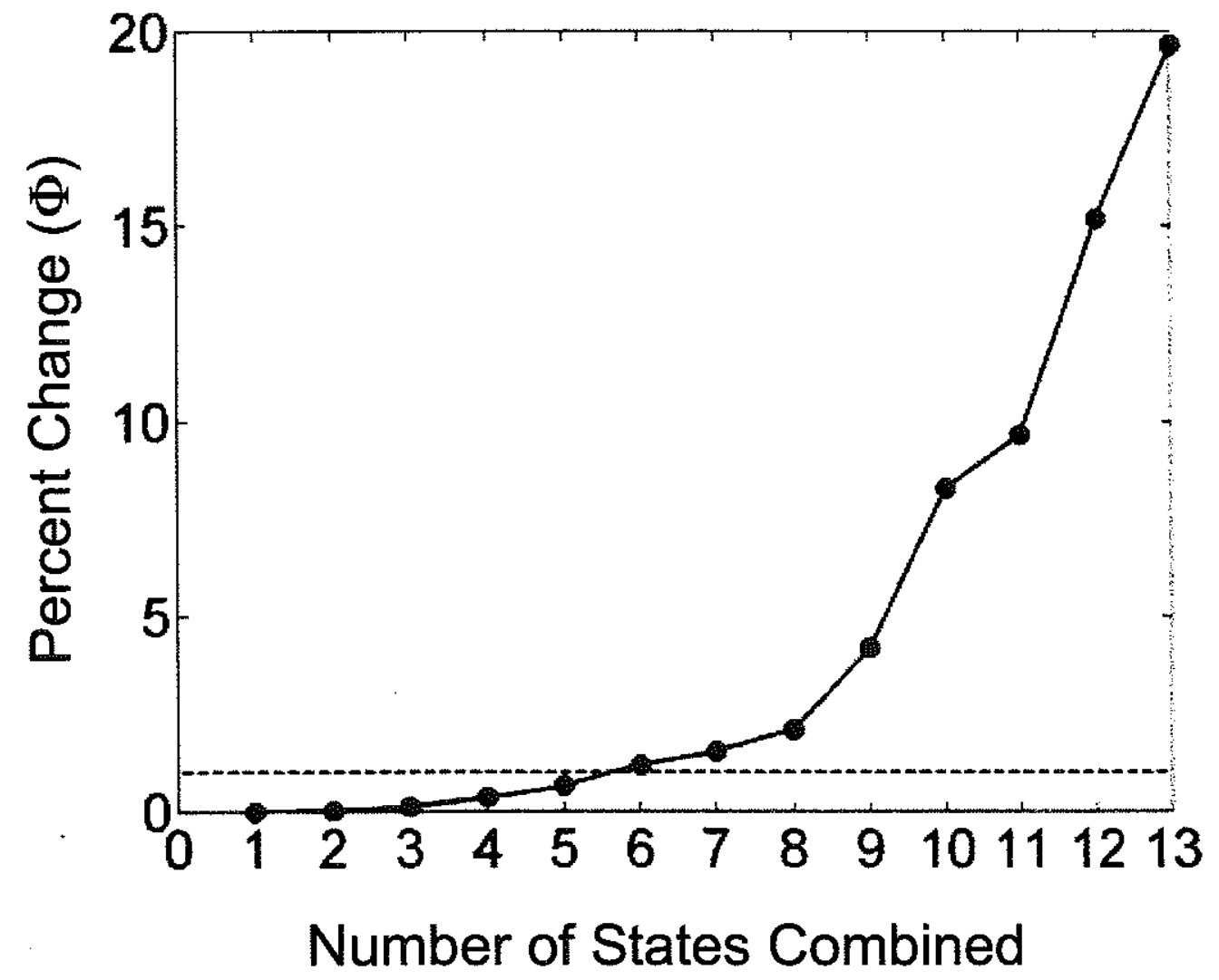

Figure 4.5: The percent change in equilibrium distribution $\left(\Phi_{w}\right)$ as a function of the reduction in the number of states in the Markov chain. The number of states were reduced by combining states in the order of their similarity. (see text for details). 


\begin{tabular}{c}
\hline \hline Functional Group (FG) \\
\hline APL \\
ASC \\
IDM \\
PAR \\
SPI \\
\hline
\end{tabular}

Table 4.3: Subtidal species that were combined into a single functional group. These species were combined because they perform functional similar roles in community dynamics (see text for details). Species are identified by the state codes used in table Tab:C4T1.

- $[\mathrm{APL} \cup \mathrm{ASC} \cup \mathrm{IDM}]$ with $[\mathrm{BR} \cup \mathrm{SPI}]$

- $[B R \cup S P I \cup A P L \cup A S C \cup I D M]$ with PAR

where $[i \cup j \cup k]$ means that the states $i, j$, and $k$ form a functional group. For our model, the compression algorithm reduces the size of the state space by combing states into a single functional group. While the threshold of $1 \%$ is somewhat arbitrary, we felt it was a good cut off point because it kept a polychaete state in our model (Filograna).

We chose to combine the states SPI, APL, ASC, IDM and PAR into a single functional group, thereby reducing the number of states in our model to 11 . We chose not to include $\mathrm{BR}$ in this grouping since it represents processes related to disturbance, and is a non-species state. We will identify the functional group species by the symbol FG (Table 4.3). The compressed transition matrix obtained by forming this functional group is shown in Table 4.4. All results throughout the remainder of the paper are based on this matrix (which we will still refer to as $\mathbf{A}$ ).

\subsubsection{Characterizing patch dynamics}

\section{Turnover rates}

The mean turnover rate of a patch is $0.3762 \mathrm{yr}^{-1}$ (Eq. 4.7). Thus on average, a patch 


\begin{tabular}{|c|c|c|c|c|c|c|c|c|c|c|c|}
\hline \multicolumn{12}{|c|}{ From } \\
\hline & $\mathrm{BR}$ & HY 1 & HY2 & MXY & MYC & MET & URT & $\mathrm{FG}$ & CRI & FIL & COR \\
\hline To:BR & 0.294 & 0.029 & 0.074 & 0.084 & 0.036 & 0.266 & 0.036 & 0.105 & 0.069 & 0.108 & 0.076 \\
\hline HY 1 & 0.101 & 0.771 & 0.056 & 0.052 & 0.017 & 0.026 & 0.009 & 0.262 & 0.145 & 0.117 & 0.056 \\
\hline HY 2 & 0.013 & 0.002 & 0.586 & 0.025 & 0.008 & 0.004 & 0.016 & 0.011 & 0.011 & 0.013 & 0.007 \\
\hline MYX & 0.025 & 0.017 & 0.026 & 0.710 & 0.006 & 0.018 & 0.012 & 0.034 & 0.031 & 0.035 & 0.028 \\
\hline MYC & 0.010 & 0.004 & 0.011 & 0.004 & 0.839 & 0.030 & 0.000 & 0.011 & 0.011 & 0.004 & 0.008 \\
\hline $\mathrm{MET}$ & 0.017 & 0.002 & 0.005 & 0.007 & 0.011 & 0.537 & 0.007 & 0.008 & 0.008 & 0.005 & 0.002 \\
\hline URT & 0.005 & 0.001 & 0.006 & 0.004 & 0.001 & 0.000 & 0.863 & 0.008 & 0.005 & 0.008 & 0.000 \\
\hline $\mathrm{FG}$ & 0.137 & 0.053 & 0.064 & 0.029 & 0.017 & 0.035 & 0.012 & 0.248 & 0.071 & 0.083 & 0.059 \\
\hline CRI & 0.320 & 0.102 & 0.147 & 0.061 & 0.054 & 0.068 & 0.024 & 0.231 & 0.609 & 0.218 & 0.222 \\
\hline FIL & 0.062 & 0.016 & 0.020 & 0.020 & 0.005 & 0.016 & 0.016 & 0.066 & 0.028 & 0.404 & 0.036 \\
\hline COR & 0.017 & 0.003 & 0.006 & 0.005 & 0.006 & 0.001 & 0.004 & 0.017 & 0.012 & 0.006 & 0.507 \\
\hline
\end{tabular}

Table 4.4: Compressed Transition matrix for the subtidal community. The compressed state FG represents the function group composed of Aplidium, Ascidia, Parasmittina, Idmidronea, and Spirorbis. State codes for the remaining species are given in table 4.1 . 
a.

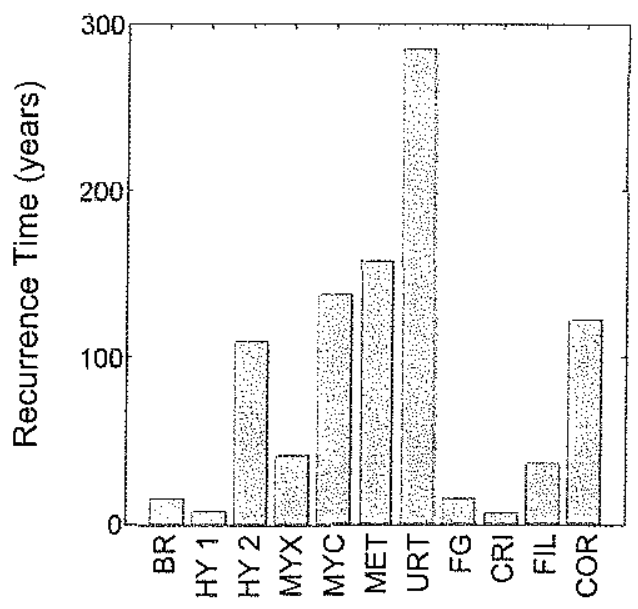

b.

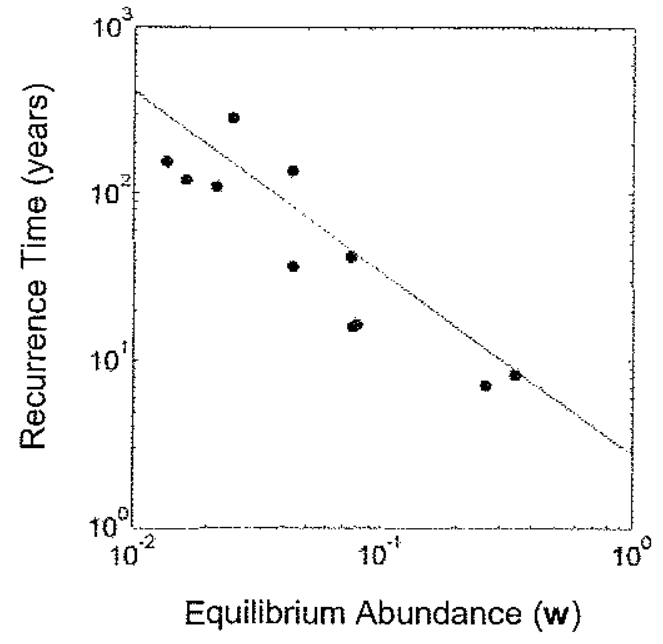

Figure 4.6: Smoluchowski recurrence times. a. Bar graph of the Smoluchowski recurrence time $\theta_{i}$ for each state in the model. The height of each bar is the expected time (in years) elapsing between a patch leaving states $i$ and then returning to it again. $b$. Smoluchowski recurrence times as a function of the equilibrium distribution of patch states w. A least square regression of the data on a log-log scale yields the power function $\theta_{i}=2.78 w_{i}^{-1.09}\left(R^{2}=0.81 p<0.001\right)$.

changes state once every 2.6 years and approximately $38 \%$ of the patches in a given quadrat change state each year.

\section{Smoluchowski recurrence time}

Figure 4.6a shows that Smoluchowski recurrence times $\theta_{i}$ are highly variable among species, ranging from 7.2 to 285.9 years. Hymedesmia 1 and Crisia have the shortest recurrence times $(\approx 8$ years $)$. The sea anemone Urticina has by far the longest recurrence time, while Metridium, Mycale, Hymedesmia 2, and coralline algae have recurrence times on the order of 100 years. Figure $4.6 \mathrm{~b}$ shows that recurrence times are highly correlated with equilibrium abundances. Thus the dominant species have the shortest return times once they are displaced from a patch.

The Smoluchowski recurrence time for bare rock, $\theta_{1}$, gives an estimation of the mean time that a patch remains occupied once it is colonized. The value of $\theta_{1}$ for 
the subtidal system is approximately 16.5 years (Fig. 4.6a). Since the mean turnover time is 2.6 years, this result suggests that after colonization a patch changes state approximately 6-7 times before it becomes unoccupied again.

\section{First Passage Times}

Figure 4.7a depicts the elements of the first passage time matrix $\mathbf{T}$. In general, there is little variation in first passage times across the rows of $\mathbf{T}$ (except for the diagonal elements $\tau_{i i}$ ), indicating that the expected time it takes for a patch to become occupied by state $i$ is independent of by the current state of the patch (given that it is not currently in state $i$ ). Figure $4.7 \mathrm{~b}$ illustrates this point by comparing the average value of the $i t h$ row of $\mathbf{T}$ (excluding $\tau_{i i}$ ) to $\theta_{i}$. The values of the two measures are almost identical suggesting that successional processes are only slightly affected by the current state of the community.

\subsubsection{Sensitivity Analysis}

The eigenvector sensitivities characterize how changes in the elements of the transition matrix affect the predicted equilibrium distribution in the community. Applying the eigenvector sensitivity formula (Eq. 4.10) to the matrix A gives the sensitivity of $\mathbf{w}$ to changes in each of the elements of A. Since there are 11 states in our model the number of eigenvector sensitivities is $11 \times 11 \times 11=1331$. Table 4.5 shows the distribution in the magnitude of the eigenvector sensitivities. The majority of them are small. Only about $4.5 \%$ of the sensitivities have magnitudes that are less than -0.25 or greater than 0.25 , suggesting that changes in the majority of transition probabilities have little effect on community composition.

The sensitivity of diversity better illustrates how changes in the transition probabilities affect community structure. Figure 4.8 shows the sensitivity of $H$ to changes in the elements of $\mathbf{A}$. We have separated positive and negative sensitivity values 
a.

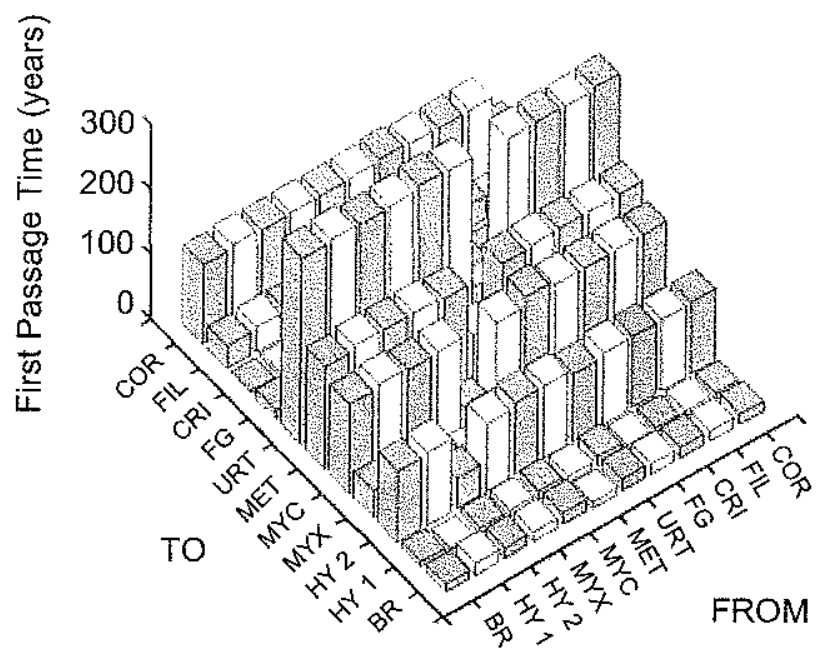

b.

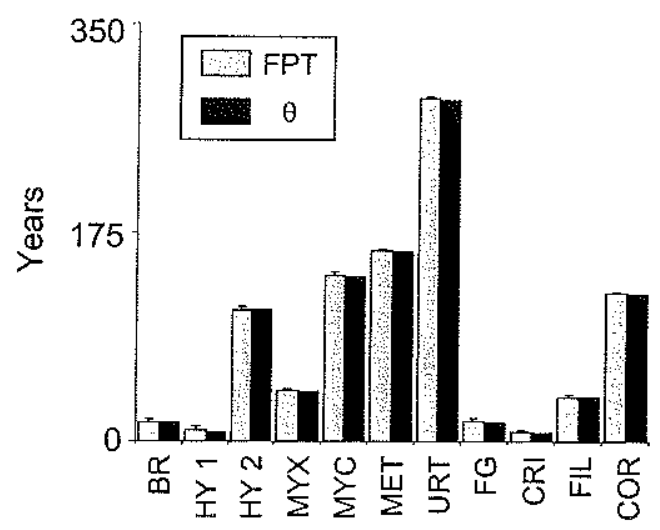

Figure 4.7: First passage times. a. A 3-D bar graph showing the value of the elements of the first passage time matrix $\mathbf{T}$. The height of each bar represents the expected time (in years) it takes a patch to go from state $j$ to states $i$. $b$. Comparison of the mean of the ith row of $\mathbf{T}$ (excluding $\tau_{i i}$ ) with $\theta_{i}$. Error bars represent $95 \%$ confidence levels of row means.

onto two graphs for visual clarity. Changes in $a_{i j}$ that have the largest positive affect on $H$ primarily involve transitions from Hymedesmia 1 and Crisia (Fig. 4.8a). Diversity is also positively sensitive to several transitions from Bare Rock (i.e. colonization probabilities), the FG functional group and Myxilla. Changes in $a_{i j}$ that have the largest negative affect on $H$ primarily involve transitions to Hymedesmia 1 and Crisia (Fig. 4.8b). Note that the magnitudes of the negative sensitivities are much less than the positive sensitivities. In general, the diversity sensitivities predict that increases in the replacement probabilities of Hymedesmia 1 and Crisia by the less dominant species (especially Urticina and Mycale) would result in the greatest increase in community diversity. 
a.

b.
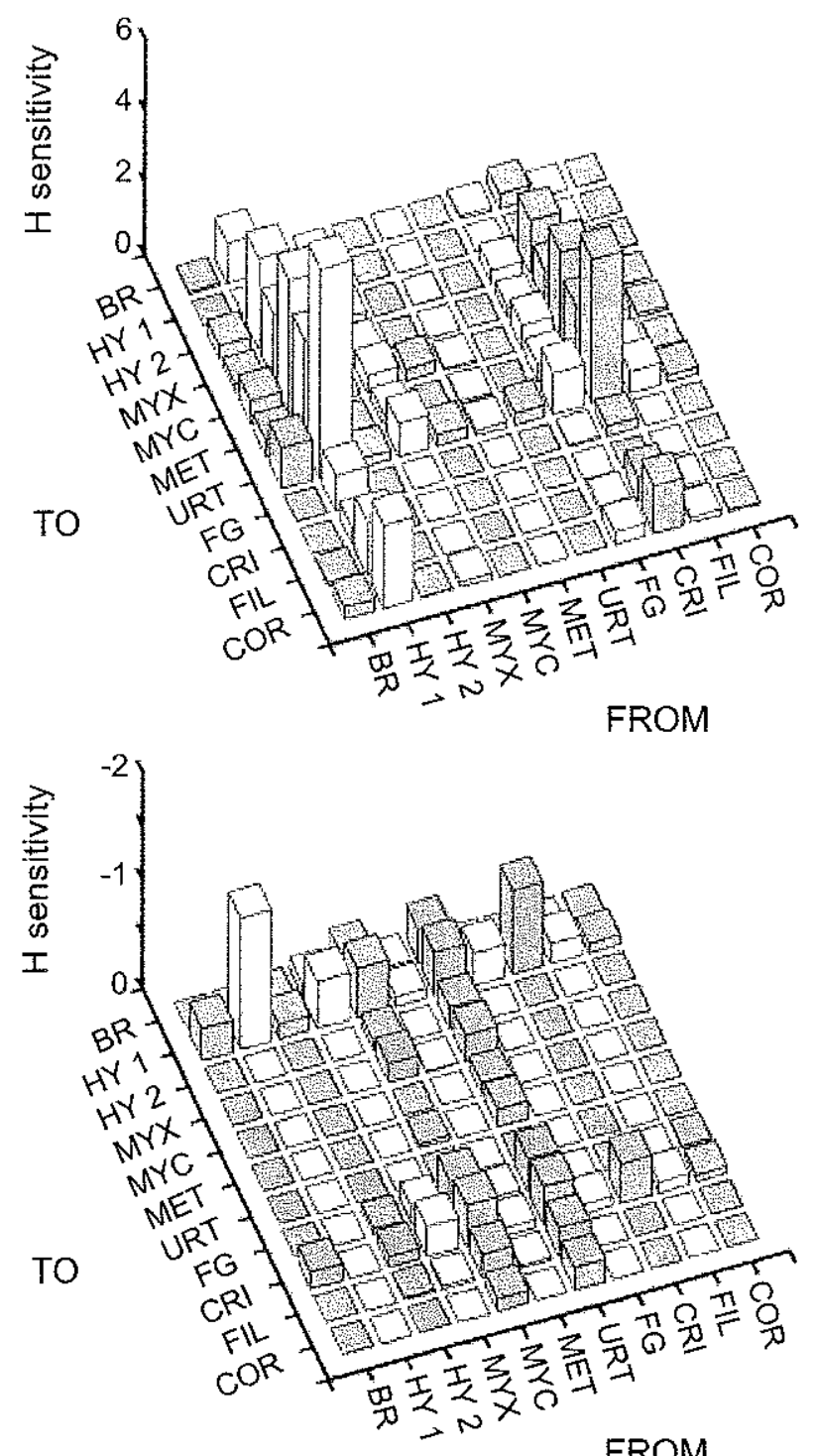

Figure 4.8: Sensitivity of equilibrium diversity $(H)$ to changes in $a_{i j}$. (a.) Positive sensitivities. Bars with large positive values represent transition probabilities that lead to greater community diversity when increased. $b$. Negative sensitivities. Bars with large negative values represent transition probabilities that lead to a reduction in community diversity when increased. 


\begin{tabular}{crr}
\hline \hline Sensitivity Range & Number & Percent \\
\hline$<-1$ & 4 & 0.30 \\
-1 to -0.25 & 21 & 1.58 \\
-0.25 to -0.1 & 60 & 4.51 \\
-0.1 to 0.1 & 1173 & 88.13 \\
0.1 to 0.25 & 39 & 2.93 \\
0.25 to 1 & 29 & 2.18 \\
$>1$ & 5 & 0.38 \\
\hline Total & 1331 & \\
\hline \hline
\end{tabular}

Table 4.5: Distribution of eigenvector sensitivities. The first column gives the range of sensitivity values, the second column is the number of sensitivities that fall within a given range, and the third column is the percent of sensitivities that fall within a given range. There are a total of 1331 eigenvector sensitivities values $(11 \times 11 \times 11)$ for our model.

\subsubsection{Species removal effects}

Figure 4.9 shows the effect of removing a species on evenness. The removal of Myxilla produces the largest decrease in $\Delta J_{i}$, however, the overall percent change in evenness is only about $5 \%$. The removal of Crisia or the functional group species (FG) also reduces evenness, resulting in a decrease in $\Delta J_{i}$ of about $3 \%$. The removal of Hymedesmia 1 , on the other hand, produces the largest increase in $\Delta J_{i}$, but again the overall change in evenness is only about $5 \%$. Thus the removal of any one species appears to have little impact on the evenness of the remaining species in the community.

Figure 4.10 shows the effect of removing a species (or bare rock) on the minimum contraction rate $\bar{\alpha}(\mathbf{A})$ and the mean contraction rate $\bar{\phi}$. The dashed lines are the values of $\bar{\alpha}(\mathbf{A})$ and $\bar{\phi}$ when all species are present. The greatest increase in $\bar{\alpha}(\mathbf{A})$ occurs when bare rock is removed, while the greatest decrease occurs when Urticina is removed. The greatest increase in $\bar{\phi}$ occurs when bare rock and Crisia are removed, while the greatest decrease occurs when Urticina and Mycale are removed. Thus the model predicts that Urticina and Mycale have a destabilizing effect on the community, while bare rock, and Crisia have a stabilizing effect on the community. 


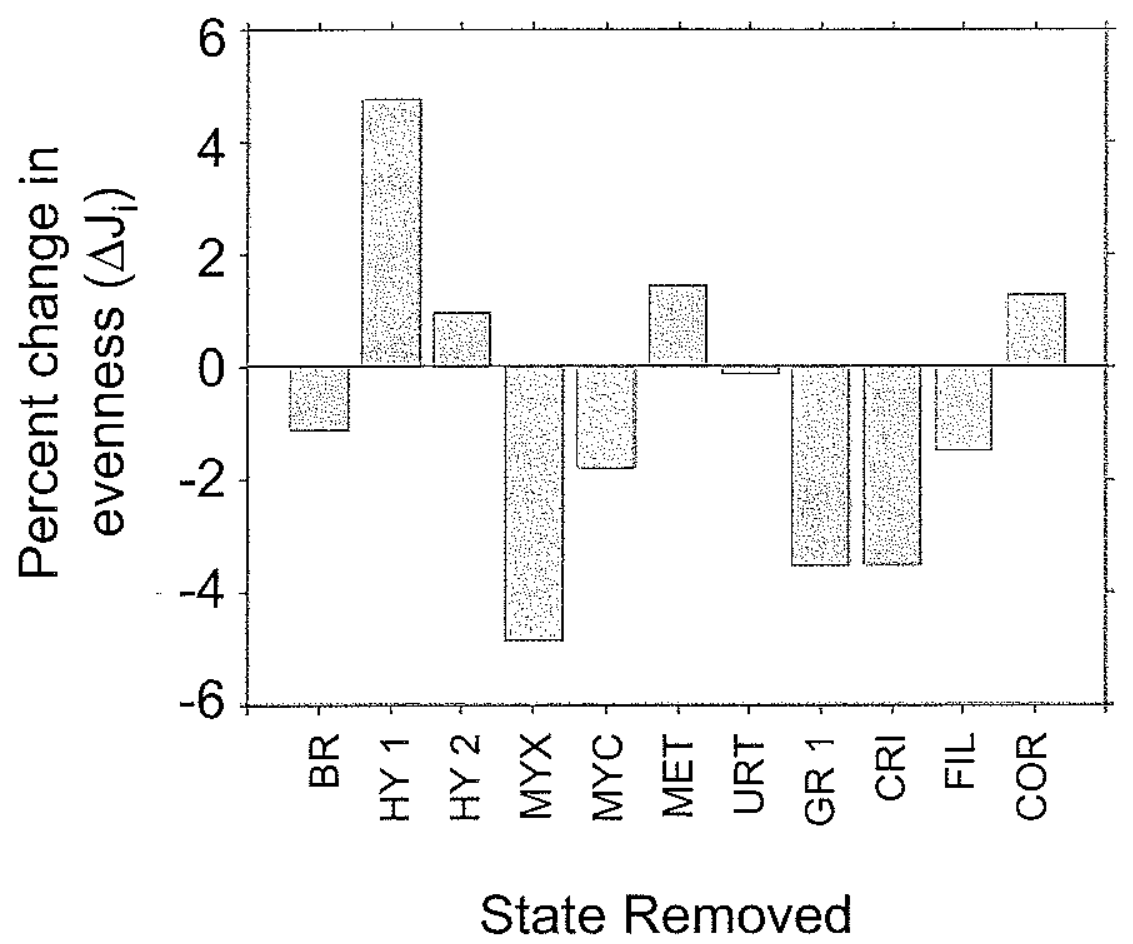

Figure 4.9: Predicted change in species evenness diversity, $\Delta J_{i}$, resulting from the removal of state $i$ from the community. 

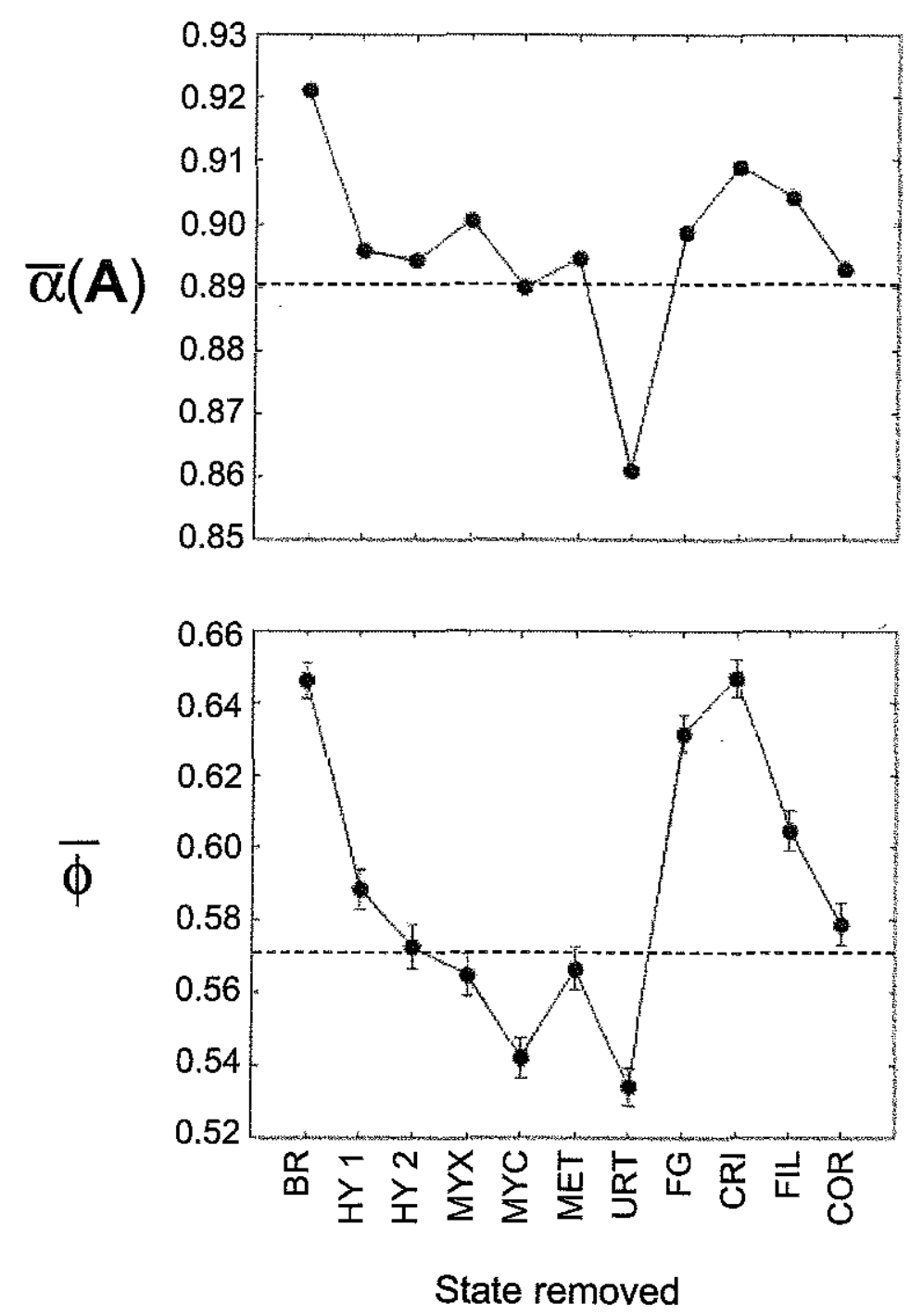

Figure 4.10: Predicted change in community resilience resulting from the removal of state $i$ from the community. $a$. Dobrushin's coefficient of ergodicity, $\bar{\alpha}(\mathbf{A})$, as a function of the removal of species $i . \bar{\alpha}(\mathbf{A})$ gives the minimum contraction rate between two probability vectors after multiplication by $\mathbf{A}$. $b$. Mean contraction rate for random pairs of probability vectors $\bar{\phi}$ as a function of the removal of species $i$. Error bars represent $95 \%$ confidence intervals. The dashed lines in the graphs are the $\bar{\alpha}(\mathbf{A})$ and $\bar{\phi}$ values for the entire subtidal community. 


\subsection{Discussion}

Markov chains are powerful tools for analyzing the dynamics of sessile communities. This paper has presented an array of analytical methods that ecologists can apply to Markov transition matrices to quantify the functional relatedness of species, to organize species into functional groups, and to characterize rates of successional change. We also introduced a method for calculating the sensitivity of the dominant eigenvector and species diversity measures to changes in species transition probabilities. Finally, we presented methods for examining the consequences of species removal on community diversity and resilience.

The transition matrix of the Markov chain provides insights into the complexity of interactions within the community. In particular, the replacement probabilities (offdiagonal elements of $\mathbf{A}$ ) provide a measure of species connectivity, sensitive to both the number and strength of species interactions. Tanner et al. (1994) have proposed that the proportion of positive off-diagonal elements of $\mathbf{A}$ is an index of the level of connectivity in a community, and have suggested that it could be used to characterize and compare different ecosystems. While we agree with their assessment, we suggest that a better measurement of connectivity would take into account both the number and relative magnitude of the positive off-diagonal elements. One possibility is to use a matrix entropy index, normalized to the number of species states in the model. Let $\hat{\mathbf{A}}$ be the transition matrix in which the column and row corresponding to bare rock have been removed (we are only interested in species replacement probabilities), with the columns renormalized so they sum to one. The normalized entropy index is

$$
E=-\left(\sum_{j}^{N} \sum_{i \neq j}^{N} \hat{a}_{i j} \ln \left(\hat{a}_{i j}\right)\right) \frac{1}{N \ln (N)}
$$

where $N$ is the number of states in $\hat{\mathbf{A}}$, and $N \ln (N))$ is the maximum entropy of the matrix. A value of $E=1$ means that all the replacement probabilities equal $1 / N$. A value of $E=0$ means that in each column of $\hat{\mathbf{A}}$ either the persistence probability, 
or one of the replacement probabilities equals 1 and all the other elements are zero. The value of the entropy index for the uncompressed subtidal transition matrix is $E=0.40$, and for the compressed matrix is $E=0.36$. Entropy values for published transition matrices of several marine and terrestrial systems are shown in Table 4.6. Calculations of Tanner et al.'s complexity ratio $(R C)$ are also shown as a comparison. There is a slight correlation between $E$ and $R C\left(r_{p}=0.52, p \approx 0.11\right)$, however, this correlation is not significant. More importantly, entropy values appear to do a better job of distinguishing between community types than $R C$. For example, three transition matrices published by Rego et al. (1993), describing the dynamics of deciduous forests subject to different fire regimes, all have $R C$ values equal to one, yet their $E$ values range from 0.43 to 0.75 (Table 4.6).

The simplest form of information obtained from a Markov chain is the steady state distribution of patches. The dominant eigenvector $(\mathbf{w})$ predicts the expected equilibrium abundance of species. In our analysis we found that predicted equilibrium abundances were in good agreement with observed species abundances within a 75 meter radius of the permanent quadrats. Several ecologists have utilized Markov chains primarily as a tool for predicting the future composition of a community (e.g. Formacion and Saila 1994; Aaviksoo 1995; Srinath 1996, Childress et al. 1998). In some cases these predictions have done a relatively poor job (e.g. Childress et al. 1998). We feel, however, the real strength of Markov chains for community ecology lies not in their ability to accurately predict the future, but in the insights they can provide into community processes.

Identifying functional groups: Spears (1998) distance metric $\delta_{i j}$ is a novel way of categorizing species into groups based on their level of functional similarity, where function is measured by the species role in community dynamics. When this metric is applied to the subtidal transition matrix, the results suggest there is a large amount of functional similarity between the polychaete Spirorbis, the ascidians Ascdia and 


\begin{tabular}{lcccl}
\hline \hline Community & Entropy (E) & Tanner's complexity ratio & Matrix size & Author \\
\hline Deciduous Forest & 0.38 & 0.85 & $5 \times 5^{*}$ & Waggoner and Stephens (1970) \\
Deciduous Forest & 0.63 & 0.80 & $11 \times 11^{*}$ & Horn (1975) \\
Deciduous Forest & & & Rego et al. (1993) \\
$\quad$ a. Biannual Spring Burn & 0.65 & 1.00 & $6 \times 6$ & \\
$\quad$ b. Biannual Autumn Burn & 0.43 & 1.00 & $6 \times 6$ & \\
$\quad$ c. Control & 0.75 & 1.00 & $7 \times 7^{*}$ & \\
Coastal vegetation & 0.21 & 0.43 & $8 \times 8$ & Gibson et al. 1997 \\
Coral Reef & & & & Tanner et al. (1994) \\
$\quad$ a. Exposed Crest & 0.21 & 0.88 & $9 \times 9$ & \\
b. Exposed Pools & 0.33 & 0.82 & $9 \times 9$ & \\
$\quad$ c. Protected Pools & 0.23 & 0.82 & $9 \times 9$ & \\
Subtidal Invertebrates & & & & Our model \\
$\quad$ a. Uncompressed Matrix & 0.40 & 0.94 & $15 \times 15$ & \\
$\quad$ b. Compressed Matrix & 0.36 & 0.97 & $11 \times 11$ & \\
\hline \hline
\end{tabular}

Table 4.6: Summary of matrix entropy $E$ values, and Tanner et al.'s complexity ratio $R C$ (defined as the number of non-zero off diagonal elements divided by the total number of off-diagonal elements) for published transition matrices. $*=$ transition matrices that do not include a bare rock (empty space) state. See text for details. 
Aplidium, and the bryozoans Parasmittina and Idmidronea. These species were all functionally similar to bare rock suggesting that they are relatively weak competitors for space.

The dendogram in Fig. 4.5 shows that it is not always wise to combine species into functional groups based solely on taxonomic considerations. For example, the sea anemone Metridium is more functionally similar to the sponges Hymedesmia 2 and Myxilla then it is to the sea anemone Urticina. This suggests that Metridium and Urticina play functional distinct roles within the subtidal communities. Incorporating these species into a single functional group would have a large effect on model behavior and could potentially obliterate important interactions.

We were able to reduce the number of states in our model by pooling similar species into functional groups using Spears' (1998) compression algorithm. This algorithm allowed us to combine states together with only a minimal loss of ecological information (less than a $1 \%$ change in the equilibrium community). In the subtidal community, the compression algorithm reduced the size of the state space by combining species into a single functional group. The reduction process, however, need not follow this pattern. If similar pairs of species cluster into distinct groupings, then the compression algorithm will organize species into multiple functional groups. The size and number of functional groups formed depends on the particularities of the species in a given community and the threshold amount of error $(\Phi)$ one is willing to accept in the model.

Rates and patterns of community change: Turnover rates and Smoluchowski recurrence times $\theta_{i}$ describe rates of succession at the spatial scale of a patch, and are useful ways of comparing the time scale of local dynamics between different types of communities. For example, we calculated turnover rates for transition matrices compiled by Tanner et. al. (1994) of three coral reef communities and obtained values of $0.10,0.20$ and 0.23 per year. For our model, we calculated a turnover rate of 0.38 
suggesting that species turnover in the subtidal community is 1.7 to 3.8 times greater than in the reef communities studied by Tanner et al (1994). Similarly, comparisons of the rate of recurrence of species within patches could be made by calculating the mean value of $\theta_{i}$ among all species $(\bar{\theta})$. For instance, in the rocky subtidal community $\bar{\theta}$ equals $81.4 \pm 23.1$ years ( 1 S.E.), while the coral reef communities have $\bar{\theta}$ values of $201.2 \pm 64.2$ years, $171.4 \pm 72.4$ years, and $190.8 \pm 100.3$ years. This suggests that the subtidal community of epifaunal invertebrates studied here is more dynamic than scleractinian coral communities on the Great Barrier Reef.

First passage times provide information on patterns of successional change. In the subtidal community, the order of species replacement is fairly independent of the species currently occupying the patch. In general there is no evidence that colonization of patches follows any kind of successional pathway (i.e. species $i \rightarrow$ species $j \rightarrow$ species $k$ ). Thus facilitation does not appear to be an important factor in community development. Instead our analysis suggests that patch succession follows an inhibition model (Connell and Slatyer 1977) in which species colonize a patch and maintain it until they die or, in some rare cases, are competitively replaced by another species (usually either Hymedesmia 1 or Crisia).

Sensitivity analysis: The eigenvector sensitivities show that changes in the majority of the transition probabilities would have little effect on the structure of the subtidal community. While only 59 of the 1331 eigenvector sensitivities had large values, there was no obvious pattern relating transition probabilities to changes in community structure. The problem with analyzing the eigenvector sensitivities in general is that there is often so many of them that their meaning is hard to interpret. The eigenvector sensitivity formula for Markov chains, however, is useful for calculating summary statistics related to the dominant eigenvector. Here we used this formula to derive an equation for the sensitivity of Shannon-Wiener diversity as a function of changes in the elements of A. Similarly, we could use equation (4.10) to calculate 
the sensitivity of several other measures of diversity. For instance, the sensitivity of Simpson's index of diversity $\left(D=1-\sum_{i} w_{i}^{2}\right)$ is $\frac{\partial D}{\partial a_{i j}}=-2 \sum_{i} w_{i} \frac{\partial w_{i}}{\partial a_{i j}}$.

The diversity sensitivities show that changes in transition probabilities associated with Hymedesmia 1 and Crisia have the greatest effect on community diversity. Because these species dominate in the community, increases in the ability of less dominant species to replace them is certain to have a positive impact on diversity. While this result is not surprising, other sensitivity predictions are less obvious. For instance, transitions from Bare Rock are predicted to have a greater effect on diversity than transitions to Hymedesmia 1 and Crisia. This pattern suggests that competition overgrowth by the two dominant species plays a relatively minor role in structuring the community compared to colonization processes. This finding is consistent with observations by Witman and Sebens (1990), who found little evidence that vegetative encroachment by Hymedesmia spp. had a significant affect on community structure in the Gulf of Maine.

Species removal: The removal of Hymedesmia 1 would produce the largest increase in species evenness $\left(\Delta J_{i}=5 \%\right)$. This is not completely surprising since Hymedesmia 1 occupies the largest proportion of patches. Figure 4.11a shows the percent change in the equilibrium abundance of each state as a result of removing Hymedesmia 1. While the abundance of all the remaining species increase, several of the less dominant species (Hymedesmia 2, Mycalle, Urticina, and coralline algae) show greater percentage increases than the dominant species Crisia. Still, the overall change in evenness is relatively small, suggesting that community composition would not change very much.

The removal of the Myxilla would produce the largest decrease in species evenness, but again the impact is small $\left(\Delta J_{i}=-5 \%\right)$. Figure $4.11 \mathrm{~b}$ shows that in the absence of Myxilla the abundance of the dominant species, Hymedesmia 1 and Crisia, would increase by about $10 \%$, while increases in the less dominant species range from $2.5 \%$ to 
a.

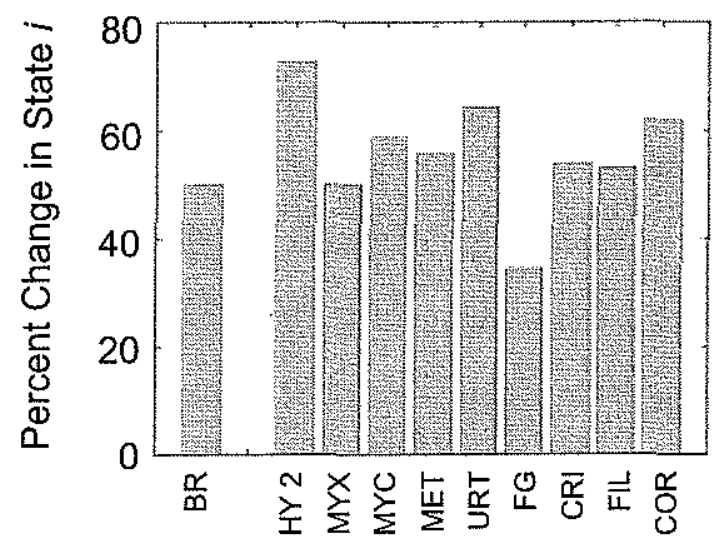

b.

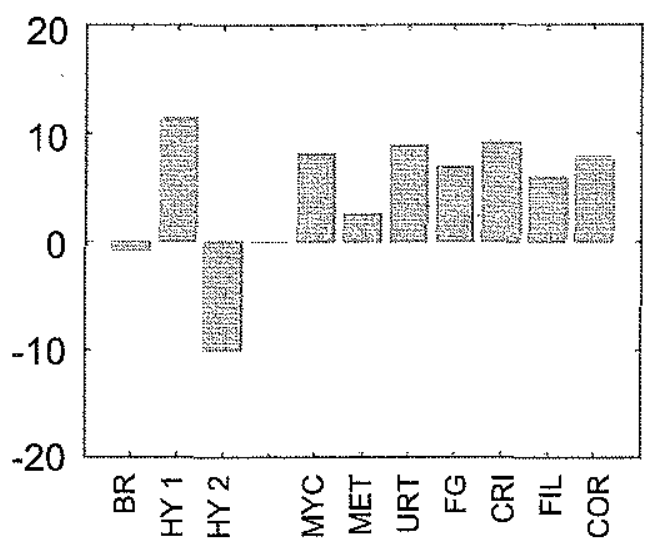

Figure 4.11: The percent change in species abundance as a as a result of removing $a$. Hymedesmia 1, and b. Myxilla from the model.

$8 \%$ (except for Hymedesmia 2) which decreases by about 10\%). Overall, the removal of any one species produces only a small change in evenness, suggesting that none of the species plays a keystone role in maintaining diversity.

The stability analyses, $\alpha(\mathbf{A})$ and $\bar{\phi}$, show that the removal of the Bare Rock state and Crisia would result in the largest decrease in the convergence rates. We can quantify the relative importance of this decrease on community resilience by estimating the time it would take two probability vectors to converge to within a distance of 0.01 (i.e. $\left\|\mathbf{x}_{1}-\mathbf{x}_{2}\right\|=0.01$ ). The maximum convergence time is calculated as $T_{m a x}=\ln 0.01 / \ln \bar{\alpha}(\mathbf{A})$, while the mean time is $\bar{T}=\ln 0.01 / \ln \bar{\phi}$. When all states are present, $T_{\max }=39.6$ years and $\bar{T}=8.2$ years. In the absence of bare rock, $T_{\max }=56.1$ years and $\bar{T}=10.6$ years, which amounts to an increase in $T_{\max }$ and $\bar{T}$ of $41.2 \%$ and $28.5 \%$ respectively. The removal of Crisia results in a $22.1 \%$ increase in $\bar{T}$ but only an $8.3 \%$ increase in $T_{\max }$. Thus, while bare rock and Crisia are both important for community resilience, bare rock appears to be more important in cases when perturbations are large. These results suggest that processes that open up space (e.g. disease, physical disturbance, predation) and processes that effect colonization 
(e.g. larval transport, regional species pool), help to stabilize the community.

While our focus here is on rocky subtidal communities, these methods are applicable to a wide range of terrestrial and aquatic communities, such as plant communities, coral reefs, or rocky intertidal communities. Markov chains are relatively easy to parameterize. Data can be collected simply by censusing quadrats at fixed intervals and recording the species occupying the space at each of a set of points. Pooling the data to estimate transition probabilities averages over small scale spatial and temporal variability and results in a homogenous Markov chain. While such models ignore the effects of temporal-, density-, and spatial-dependence, analyses of homogenous Markov chains can provide important insights into successional processes and the effects of species interactions on community structure. To assess the importance of stochastic and nonlinear effects, non-homogenous Markov models can be developed which reflect the temporal-, density-, and spatial-dependent realities of the community (Hill et al. in prep.). Whether including these higher-level effects provides new insights into the role of species in a community, however, remains to be determined. 


\subsection{References}

Aaviksoo K. 1995. Simulating vegetation dynamics and land use in a mire landscape using a Markov model. Land. Urban Plann., 31:129-142.

Acevedo, M. 1982. On Horn's Markovian model of forest dynamics with particular reference to tropical forests. Theoretical Population Biology, 19:230-250.

Ayala, F.J. 1976. Molecular Evolution. Sinauer, Sunderland, MA.

Ayling, A.M. 1981. The role of biological disturbance in temporal subtidal encrusting communities. Ecology, 62:830-847.

Bond, W.J. 1994. Keystone species. In E.D. Schulze and H.A. Mooney, Biodiversity and Ecosystem Function. Springer-Verlag, Berlin, pp. 237-253.

Bruno, F. and J.D. Witman. 1996. Defensive mechanisms of scleractinian cup corals against overgrowth by colonial invertebrates. J. Exp. Mar. Biol. Ecol., 207:229241 .

Buss, L.W and J.B.C. Jackson. 1979. Competitive networks: nontransitive competitive relationships in cryptic coral reef environments. Am. Nat, 113:223-234.

Caswell H. 1989. Matrix population models. Construction, analysis, and interpretation. Sinauer Associates Inc., Sunderland, MA.

Caswell H. 2000. Matrix population models. Construction, analysis, and interpretation. Sinauer Associates Inc., Sunderland, MA. 2nd Ed., in press.

Caswell H., and J.E. Cohen. 1991a. Communities in patchy environments: a model of disturbance, competition, and heterogeneity. In J. Kolasa and S.T.A. Pickett (eds.) Ecological Heterogeneity. Springer-Verlag, New York, pp. 97-122.

Caswell H., and J.E. Cohen. 1991b. Disturbance, interspecific interaction and diversity in metapopulations.Biol. J. Linnean Soc., 42:193-218. 
Childress, W.M., Crisafulli, C.M., and Rykiel Jr., E.J. 1998. Comparison of Markovian matrix models of a primary successional plant community. em Ecological Modeling, 107:93-102.

Connell J.H. 1961a. The effects of competition, predation by Thais lapillus and other factors on natural populations of the barnacle Balanus balanoides. Ecol. Monogr., 31:61-104.

Connell J.H. 1961b. The influence of interspecific competition and other factors on the distribution of the barnacle Chthamalus stellatus. Ecology, 42:710-723.

Connell J.H. 1972. Community interactions on marine rocky intertidal shores. Ann. Rev. Eco. Sys., 3:169-192.

Connell J.H. 1983. On the prevalence and relative importance of interspecific competition: evidence from field experiments. Am. Nat., 122:666-696.

Connell J.H., and R.O. Slatyer. 1977. Mechanisms of succession in natural communities and their roles in community stability and organization. Am. Nat., 111:11191144.

Connell, J.H. and M.J. Keough. 1985. Disturbance and patch dynamics of subtidal marine animals on hard substrata. In S.T.A. Picket and P.S. White (eds.), The ecology of natural disturbance and patch dynamics. Academic Press, Orlando, pp. 125-147.

Dayton P.K. 1971. Competition, disturbance, and community organization: the provision and subsequent utilization of space in a rocky intertidal community. Ecological Monographs, 41:351-389.

Dayton, P.K., G.A. Robilliard and R.T. Paine. 1970. Benthic faunal zonation as a result of anchor ice at McMurdo Sound, Antarctica. In Antarctic ecology. Martin Holdgate ed., Academic Press, London 
Dobrushin, R.L. 1956a. Central limit theorem for nonstationary Markov chains, I. Theory of Probability and its Application, 1:65-80.

Dobrushin, R.L. 1956b. Central limit theorem for nonstationary Markov chains, II. Theory of Probability and its Application, 1:329-383.

Duggins, D.O. 1983. Starfish predation and the creation of mosaic patterns in a kelp dominated community. Ecology, 64:1610-1619.

Formacion S.P., and S.B. Salia. 1994. Markov chain properties related to temporal dominance changes in a Philippine pelagic fishery. Fish. Res., 19:241-256.

Gaines, S.D. and M.D. Bertness. 1992. Dispersal of juveniles and variable recruitment in sessile marine species. Nature, 360:579-580.

Genovese, S.J. 1996. Regional and temporal variation in the ecology of an encrusting bryozoan in the Gulf of Maine. Ph.D. Dissertation, Northeastern University.

Genovese, S.J. and J.D. Witman. 1999. Interactive effects of flow speed and particle concentration on the growth rates of an active suspension feeder. Limnology and oceanography, 44;1120-1131.

Gibson, D.J., J.S. Ely and P.B. Looney. 1997. A Markovian approach to modeling succession on a coastal barrier island following beach nourishment. Journal of Coastal Research, 13, 831-841.

Goldwasser, L. and J. Roughgarden 1993. Construction and analysis of a large Caribbean food web. Ecology, 74:1216-1233.

Graham, K.R. and K.P. Sebens. 1996 The distribution of marine invertebrate larvae near vertical surfaces in the rocky subtidal zone. Ecology 77:933-949

Grant, W.E., J.H. Matis, and W. Miller. 1988. Forecasting commercial harvest of marine shrimp using a Markov chain model. Ecol. Model., 45:183-193. 
Horn, H.S. 1975. Markovian properties of forest succession. pp. 196-211 in M.L. Cody and J.M. Diamond (eds.) Ecology and evolution of communities. Harvard University Press, Cambridge, MA.

Hughes, T.P. 1994. Catastrophes, phase shifts, and large-scale degradation of a Caribbean coral reef. Science, 265:1547-1551.

Iosifescu M. 1980. Finite Markov processes and their applications. Wiley, New York. Isagi Y., and N. Nakagoshi. 1990. A Markov approach for describing post-fire succession of vegetation. Ecol. Res., 5:163-171.

Jackson J.B.C. 1977. Competition on hard marine substrata: the adaptive significance of solitary and colonial strategies. Am. Nat., 111:743-767.

Jackson J.B.C. 1991. Adaptation and diversity of reef corals. Bioscience, 41:475-481.

Keough, S.J. 1983. Patterns of recruitment of sessile invertebrates in two subtidal communities. Journal of Experimental Marine Biology and Ecology, 66:213-245.

Laska, M.S. and T. Wootton. 1998. Theoretical concepts and empirical approaches to measuring interaction strength. Ecology, 79:461-476.

Leichter, J.J. and J.D. Witman. 1997. Water flow over subtidal rock walls: relationship to distribution and growth rate of sessile suspension feeders in the Gulf of Maine. Journal of Experimental Marine Biology and Ecology, 209:293-307.

Lloyd, M. and R.J. Ghelardi. 1964. A table for calculating the equability component of species diversity. J. Anim. Ecol., 33:217-225.

Macarthur, R.H. 1972. Strong or weak interactions? Transactions of the Connecticut Academy of Arts and Science, 44:177-188.

Masaki, T., W. Suzuki, K. Niiyama, S. Iida, H. Tanaka, and T. Nakashizuka. 1992. Community structure of a species-rich temperate forest, Ogawa Forest Reserve, central Japan. Vegetatio, 98:97-111. 
Menge, B.A. 1976. Organization of the New England rocky intertidal community: role of predation, competition and environmental heterogeneity. Ecological Monographs, 46:355-393.

Menge B.A. and J.P. Sutherland. 1976. Species diversity gradients: Synthesis of the roles of predation, competition, and temporal heterogeneity. Am. Nat, 110:351369 .

Menge, B.A., E.L. Berlow, C.A. Blanchette, S.A. Navarrete, and S.B. Yamada. 1994. The keystone species concept; variation in interaction strength in a rocky intertidal zone. Ecological Monographs, 64:249-286.

Osman R.W. 1977. The establishment and development of a marine epifaunal community. Ecol. Monogr., 47:37-63.

Paine R.T. 1966. Food web complexity and species diversity. Am. Nat., 100:65-75.

Paine R.T. 1974. Intertidal community structure: Experimental studies on the relationship between a dominant competitor and its principle predator. Oecologia, 15:93-120.

Paine, R.T. 1976. Controlled manipulations in the marine intertidal zone, the their contributions to ecological theory. In The changing scene in the natural sciences. Special publication, Academy of Natural Sciences, 12:245-270.

Paine, R.T. 1980. Food webs: linkage, interaction strength and community infrastructure. The third Tansley lecture. Journal of Animal Ecology, 49:667-685.

Paine, R.T. 1992. Food-web analysis through field measurement of per capita interaction strengths. Nature, 355:73-75.

Paine R.T., and S.A. Levin. 1981. Intertidal landscapes: disturbance and the dynamics of pattern. Ecol. Monogr., 51:145-178. 
Quinn J.F. 1982. Competitive hierarchies in marine benthic communities. Oecologia, 54:129-125.

Rego, F., Pereira, J. and Trabaud, L. 1993. Modeling community dynamics of a Quercus coccifera L. garrigue in relation to fire using Markov chains. Ecological Modeling, 66:251-260.

Roughgarden J., S. Gaines, and H. Possingham. 1988. Recruitment dynamics in complex life cycles. Science, 241:1460-1466.

Runkle, J.R. 1981. Gap generation in some old growth forests of the eastern United States. Ecology, 62:1041-1051.

Russ, G.R. 1982. Overgrowth in a marine epifaunal community: competition hierarchies and competitive networks. Oecologia, 53:12-19.

Salia, S.B., and K. Erzini. 1987. Empirical approach to multispecies stock assessment. Trans. Am. Fish. Soc., 116:601-611.

Scheibling, R.E. and A.W. Hennigar 1997. Recurrent outbreaks of disease in sea urchins Strongylocentrotus droebachiensis in Nova Scotia: evidence for a link with large-scale meterologic and oceanographic events. Marine Ecology Progress Series, 152:155-165.

Sebens K.P. 1985. The ecology of the rocky subtidal zone. American Scientist, 73:548-557.

Sebens K.P. 1986. Spatial relationships among encrusting marine organisms in the New England subtidal zone. Ecol. Monogr., 56:73-96.

Shannon C.E., and W. Weaver 1963. The mathematical theory of communication. University of Illinois Press, Urbana, Il.

Smith, F. and J.D. Witman. 1999. Species diversity in subtidal landscapes: Maintenance by physical processes and larval recruitment. Ecology, 80:51-69. 
Sousa, W.P. 1985. Disturbance and patch dynamics on rocky intertidal shores. In S.T.A. Picket and P.S. White (eds.), Natural Disturbance: the patch dynamics perspective. Academic Press, NY.

Spears W.M. 1998. A compression algorithm for probability transition matrices. SIAM J. Matrix Anal. Appl., 20:60-77.

Srinath, M. 1996. Markov chain applications to the dynamics of the pelagic fishery along the Kerala coast, India. Indian J. Fish., 43:115-120.

Steneck, R.S. 1982. A limpet-coralline alga association: adaptations and defenses between a selective herbivore and its prey. Ecology 63:507-522.

Steneck, R.S. 1997. Fisheries induced biological changes to the structure and function of the Gulf of Maine ecosystem. In G.T. Wallace and E.F. Braasch (eds.), Proceedings of the Gulf of Maine ecosystem dynamics. RARGOM report 97-1. Hanover, NH.

Sutherland J.P. 1974. Multiple stable points in natural communities. Am. Nat., 108:859-873.

Tanner J.E., T.P. Hughes and J.H. Connell. 1994. Species coexistence, keystone species, and succession: a sensitivity analysis. Ecology, 75:2204-2219.

Tanner J.E., T.P. Hughes and J.H. Connell. 1996. The role of history in community dynamics: a modeling approach. Ecology, 77:108-117.

Underwood, A.J. and E.J. Denley. 1994. Paradigms, explanations and generalizations in models for the structure of intertidal communities on rocky shores. In D.R. Strong, D. Simberloff, L.G. Abele and A.B. Thistle. (eds.), Ecological Communities: conceptual issues and the evidence. Princeton University Press, Princeton.

Usher, M.B. 1979. Markovian approaches to ecological succession. Journal of Animal Ecology 48:413-426. 
Vadas, R.L. and R.S. Steneck. 1988. Zonation of deep water benthic algae in the Gulf of Maine. Journal of Phycology, 24:338-346.

Vance, R.R. 1978. A mutualistic interaction between a sessile marine clam and its epibionts. Ecology, 59:679-685.

Velimirov, B. and C.L. Griffiths. 1979. Wave induced kelp movement and its importance for community structure. Botanica Marina, 22:169-172.

Waggoner P.E., and G.R. Stephens. 1970. Transition probabilities for a forest. $\mathrm{Na-}$ ture, 255:1160-1161.

Watling, L and E.A. Norse. 1998. Disturbance of the Seabed by Mobile Fishing Gear: A Comparison to Forest Clearcutting. Conservation Biology, 12:1180-1197.

Witman J.D. 1985. Refuges, biological disturbance, and rocky subtidal community structure in New England. Ecol. Monogr., 55:421-445.

Witman J.D. 1987. Subtidal coexistence: storms, grazing mutualism, and the zonation of kelp and mussels. Ecol. Monogr., 57:167-187.

Witman J.D. 1996. Dynamics of Gulf of Maine benthic communities. pp 51-69 in D. Dow and E. Braasch (eds.) The health of the Gulf of Maine ecosystem: cumulative impacts of multiple stressors. RARGOM Report 96-1, Hanover, NH.

Witman, J.D. and R.A. Cooper. 1983. Distribution and contrasting patterns of population structure in the brachiopod Terebratulina septentrionalis from two subtidal habitats. J. Exp. Mar. Biol. Ecol., 73:57-79.

Witman, J.D. and K.P. Sebens. 1988. Benthic community structure at a subtidal rock pinnacle in the central Gulf of Maine. In I. Babb and M. DeLuca (eds.), Benthic Productivity and Marine resources of the Gulf of Maine. National Undersea Research Program report, 88:67-104. 
Witman, J.D. and K.P. Sebens. 1990. Distribution and ecology of sponges at a subtidal rock ledge in the central Gulf of Maine. PP. 391-396 in K. Rutzler (ed.) New perspectives in sponge biology. Smithsonian Institution Press, Washington, D.C.

Witman, J.D. and K.P. Sebens 1992. Regional variation in fish predation intensity: a historical perspective in the Gulf of Maine. Oecologia, 90:305-315.

Witman, J.D. and K.R. Grange. 1998. Links between rain, salinity and predation in a rocky subtidal community. Ecology, 79:2429-2447.

Witman, J.D. and P.K. Dayton. 2000. Rocky subtidal communities. In Bertness, M.D. and M. Hay eds. Marine Benthic Ecology. Sinauer Press (in press)

Wootton J.T. 1993. Indirect effects and habitat use in an intertidal community: Interaction chains and interaction modifications. Am. Nat., 141:71-89. 


\section{Chapter 5}

\section{Temporal and spatial variation in successional patterns in a rocky subtidal community: Statistical methods, Markov chain models, and Species distributions}

Consistency is contrary to nature, contrary to life.

-Aldous Huxley

\subsection{Introduction}

Subtidal rock surfaces in New England support highly diverse communities of sessile invertebrates that form a tight patchwork of species bound to the hard substrate (Sebens 1985; Witman and Dayton 2000). Several processes are thought to be important in maintaining subtidal diversity, include predation (Ayling 1981; Duggins 1983; Witman and Cooper 1983), disease (Scheibling and Hennigar 1997) competition (Osman 1977, Sebens 1986), physical disturbance (Dayton et al 1970, Witman 1987, Witman and Dayton 2000), recruitment (Smith and Witman 1999), sedimentation (Witman and Dayton 2000), and current flow (Genovese 1996, Genovese and

\footnotetext{
${ }^{1}$ This chapter been submitted to Ecology Letters for publication.
} 
Witman 1999). While the rates of these processes can vary over a range of spatial and temporal scales (Witman 1995), there is little information about the effect this variation has on successional dynamics and community composition.

Hill et al.(2000) recently developed a Markov chain model of subtidal community dynamics, based on observations of species succession in ten replicate quadrats monitored over an eight-year period. 'To model the community, we subdivided quadrats into an array of small patches, each of which is in one of a number of possible states. Species or groups of species were identified with states of the Markov chain, and the course of succession within patches was defined by a transition matrix A whose $(i, j)$ entry gives the probability that a patch in state $j$ changes to state $i$ in one time step.

The model developed by Hill et al. (2000) is a homogenous Markov chain. It averages transition probabilities over small-scale spatial and temporal variability to produce the best single realization of a Markov chain for the subtidal community. Patch transition probabilities, however, characterize ecological processes (e.g., colonization, disturbance, competition, predation, persistence) that are affected by changes in environmental conditions. Because homogenous Markov models ignore the effects of spatial and temporal variation on transition probabilities, they are inadequate to evaluate the effects of environmental change on successional patterns.

The transition data collected by Hill et al. can be partitioned into categories corresponding to specific locations (i.e. quadrat) and time intervals. If multiple transition matrices are estimated from counts of observed patch transitions at different spatial locations and at different times, then the effects of temporal and spatial variability on successional processes can be tested using log-linear models (Bishop et al. 1975, Caswell 1989, 2000). These models are the more powerful descendants of the Anderson and Goodman (1957) model, which was original proposed to test the timehomogeneity of Markov chains. The Anderson and Goodman (1957) test has been applied to community transition matrices (Tanner et al. 1994; Wootton 2000), however, the log-linear approach is more general and can be extended to more complex 
experimental designs (Caswell 2000).

Temporal and spatial variability of the environment not only affects the successional dynamics of patches, but also the regional dynamics of the community. We can characterize the effects of this variability on regional dynamics by examining how the relative abundance of subtidal species varies over time and space. This method was proposed by Cohen et al. (1998), and involves constructing a series of probability matrices describing the distribution of each species among quadrats through time. As far as we know, this method has never been applied to an actual data set.

In this paper we report on the use of log-linear analysis to test for significant effects of temporal and spatial variation on patch transition probabilities in the subtidal community. We use the information from this analysis as a guide for developing sets of Markov transition matrices to predict mean species abundances and to characterize successional dynamics. We also examine the effects of temporal and spatial variation on regional dynamics by constructing probability matrices describing the distribution of species in space and time. These matrices are analyzed to quantify how the spatial distribution of each species varies as a function of time, and how the temporal distribution of each species varies as a function of quadrat location (Cohen et al. 1998).

\subsection{Methods}

\subsubsection{Data collection}

The focus of our study is a community of sessile invertebrates (sponges, sea anemones, polychaetes, bryozoans, ascidians) and crustose coralline algae living on rock walls at 30 - $33 \mathrm{~m}$ depth on Ammen Rock Pinnacle in the central Gulf of Maine. The data consists of a series of photographs of ten permanent quadrats (each $30 \times 20 \mathrm{~cm}^{2}$ in area) chronicling the spatial distribution of species and bare rock over a eight year period (1986 - 1994). The permanent quadrats were photographed at least annually 


\begin{tabular}{l|lc}
\hline \hline Model States & \multicolumn{3}{|l}{ Species Type } & State Codes \\
\hline Bare Rock & & BR \\
\hline Hymedesmia $1 \mathrm{sp}$. & Sponge & HY 1 \\
\hline Hymedesmia $2 \mathrm{sp}$. & Sponge & HY 2 \\
\hline Myxilla fimbriata & Sponge & MYX \\
\hline Mycale lingua & Sponge & MYC \\
\hline Metridium senile & Sea anemone & MET \\
\hline Urticina crassicornis & Sea anemone & URT \\
\hline Aplidium pallidum & Ascidian & \\
Ascidia callosa & Ascidian & \\
Parasmittina jeffreysi & Bryozoan & FG \\
Idmidronea atlantica & Bryozoan & \\
Spirorbis spirorbis & Polychaete & \\
\hline Crisia eburnea & Bryozoan & CRI \\
\hline Filograna implexa & Polychaete & FIl \\
\hline Coralline Algae & Encrusting algae & COR \\
\hline \hline
\end{tabular}

Table 5.1: Subtidal species identified in the nine quadrats located at 30 meters depth on Ammen Rock pinnacle in the Gulf of Maine. Species are identified in the model using the state codes in the left-hand column.

with a Nikonos V camera equipped with a $15 \mathrm{~mm}$ lens and two strobes mounted on a rigid camera frame (quadrapod) as described in Witman (1985). Within a sampling period, each quadrat was treated as an independent replicate, because individual quadrats were separated by a horizontal distance of 1 to 5 meters.

A total of 14 species, each occupying at least $0.5 \%$ of the study area, were recorded in the quadrats. We grouped these species into 11 different state categories (table 5.1). We chose to combine the ascidians, Aplidium and Ascidia, the bryozoans, Parasmittina and Idmidronea, and the polychaete Spirorbis into a single functional group based on an objective analysis showing a high level of functional similarity among these species. Function similarity is measured by the role these species play in community dynamics (see Hill et al. 2000 for details).

Transition data were obtained by superimposing a lattice of evenly spaced points 
over quadrat photographs and following the state of each point through time. Approximately 600 points (a single point every $1 \mathrm{~cm}$ ) were assayed per quadrat. We chose this scale because it was approximately equivalent to the size of the smallest organism in our data set. For simplicity we will refer to each point as a patch, where the size of a patch is taken to be $1 \mathrm{~cm}^{2}$. Since individuals from many of the subtidal species are capable of growing much larger than $1 \mathrm{~cm}^{2}$, a single individual can occupy more than one patch.

\subsubsection{Statistical Analysis}

The observed transition data form a four-way contingency table $\mathbf{N}$, in which patches are classified by their state $(S)$ at time $\mathrm{t}$, their fate $(F)$ at time $\mathrm{t}+1$, the time $(T)$ of the observation, and the location $(L)$ of the quadrat. The entry $n_{i j k l}$ in cell $(i j k l)$ of the table gives the number of patches making the transition from state $i$ to fate $j$ at time $k$ in location $l$ (Caswell 2000).

To evaluate the significance of temporal and spatial heterogeneity in the transition data, we used log-linear analysis (Bishop et al. 1975, Caswell 1989, 2000). In loglinear analysis the logarithm of the cell frequencies of $\mathbf{N}$ is modeled as a linear function of the effects of $F, S, T, L$, and their interactions. The significance of the effects of time, location, and their interaction are evaluated by comparing the likelihood of models including progressively more interactions.

The null hypothesis in tests for differences among Markov chains is one of conditional independence: given the initial state $(S)$, the fate $(F)$ of a patch is independent of time and location (Bishop et al. 1975). Because we consider only hierarchical models, the null model is denoted FS, STL; which implies that the cell frequencies are modeled as a function of the effects of $F S$ and $S T L$, plus the effects of $S T, S L, T L$, $S, T$, and $L$ (see the appendix for details).

The effects of time and location on transition probabilities can be tested in several ways as shown in figure 5.1. Beginning with the null hypothesis $F S, S T L$ at the top 
of the figure, we add location effects by including the terms $F L$ and FSL. Their effect is evaluated by comparing the models $F S, S T L$ and $F S L, S T L$. Similarly, we add time effects to the null model by including the terms $F T$ and $F S T$ and evaluate their effects by comparing the models $F S, S T L$ and FST,STL (Caswell 2000).

We can also test for the effects of location by adding the terms $F L$ and $F S L$ to the model that includes the time effects and comparing the models FSL,STL and $F S L, F S T, S T L$. Similarly, we can test for the effects of time by adding the terms $F T$ and FST to the model that includes the location effects and comparing the models $F S T, S T L$ and $F S L, F S T, S T L$. Finally, we test for the effects of time and location by comparing the models FSL, FST, STL and FSTL (Caswell 2000).

The goodness-of-fit of a model is measured using the log-likelihood ratio statistic $G^{2}$ which is asymptotically distributed as $\chi^{2}$ with degrees of freedom equal to the difference between the number of cells in $\mathbf{N}$ and the number of parameters in the model. The goodness-of-fit test compares a model to the saturated model FSLT, which fits the data exactly. Tests of a specific interaction are assessed by examining the difference in $G^{2}$ values $\left(\Delta G^{2}\right)$ between models that include and exclude that interaction. The likelihood statistic $\Delta G^{2}$ is distributed as $\chi^{2}$ with degrees of freedom equal to the difference between the degrees of freedom for the two models (Caswell 2000).

Increasing the number of parameters in a log-linear model will always produce a better fit to the data. To determine which model best approximates the mechanisms generating the transition data we used the Akaike Information Criteria (AIC)(Akaike 1973; Burnham and Anderson 1998). For log-linear models AIC is defined as

$$
A I C=G^{2}-2(d f)
$$

(Christensen 1990) where $G^{2}$ is the goodness of fit likelihood statistic and $d f$ is the degrees of freedom of the model. The log-linear model that minimizes AIC is considered the most parsimonious, best-fitting model (Burnham and Anderson 1998). 


\subsection{Results: The effects of time and location on patch succession}

A graphic representation of the results of statistical tests for the effects of time and location on transition data in the subtidal community is shown in Figure 5.1. The effect of location is highly significant whether it is evaluated with the effect of time excluded or included in the analysis. Time is also highly significant whether or not location is excluded or included in the analysis. The interaction of time and location, however, is not significant $(\mathrm{P}=0.998)$.

Comparison of AIC values shows that the best model is FST, FSL, STL (AIC = -7566.2). Thus both location and time have important affects on patch transitions in rocky subtidal communities. Incorporation of a time $\times$ location interaction, however, is unnecessary to explain observed patch transition patterns. Note that the model $F S T, F S L$ has a much lower $A I C$. value than $F S T, F S T$, indicating that location has a larger effect on transition probabilities than time.

\subsection{Markov chain models: Species composition and community dynamics}

Species composition and successional dynamics in the rocky subtidal community can be studied using Markov chain models. The log-linear analysis suggests that these models should incorporate the variability in patch transitions associated with time and location, without including a time $\times$ location interaction. To explore the implication of these results we constructed three sets of transition matrices: 1 . a set of 10 spatial matrices in which the effects of time are ignored, 2. a set of 8 time-varying matrices in which the effects of space are ignored, and 3 . a set consisting of a single homogeneous matrix in which temporal and spatial effects are ignored.

Let $\mathcal{S}$ represent the set of spatially-varying transition matrices, and let $\mathbf{S}^{(l)}$ denote 


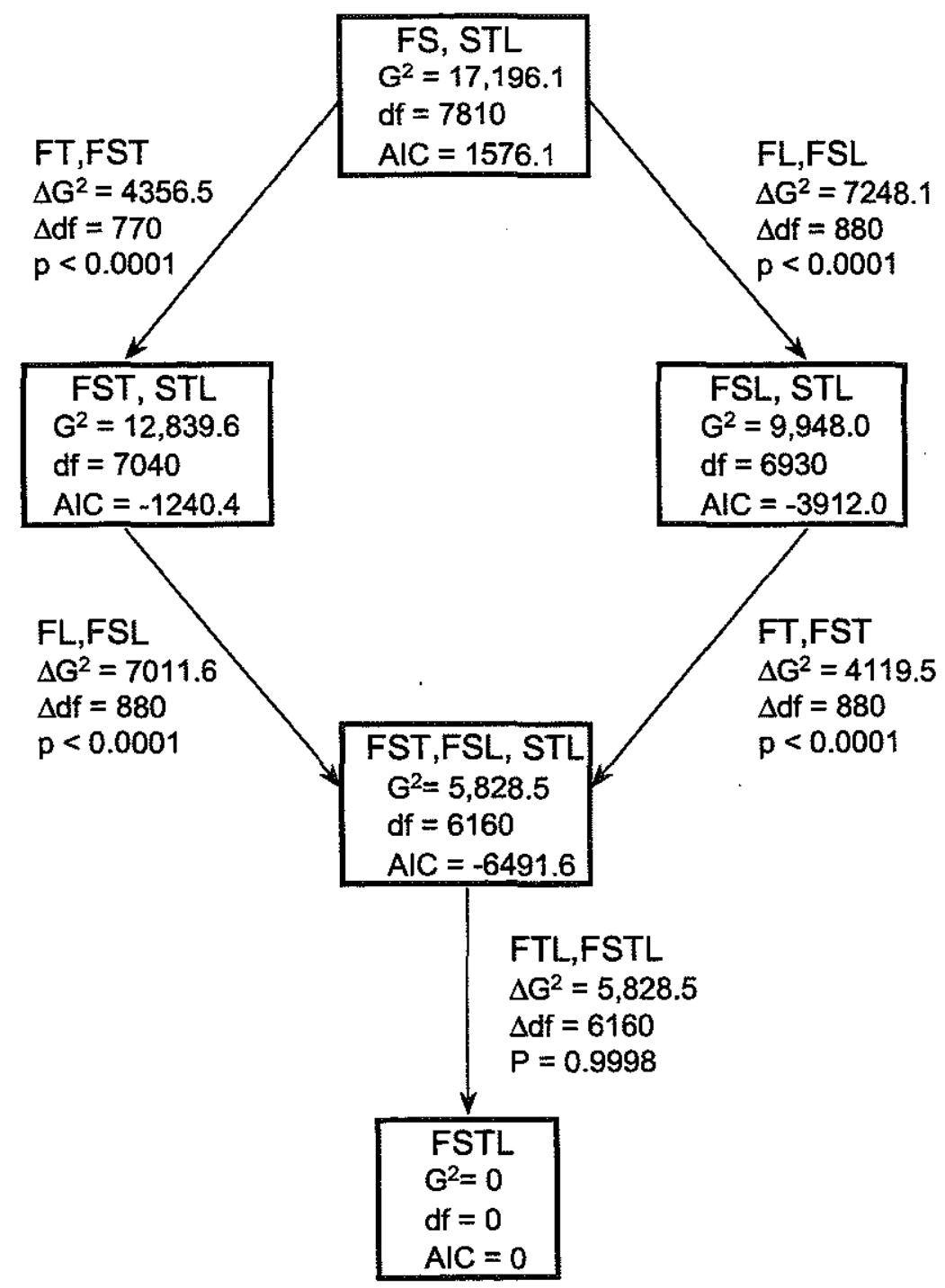

Figure 5.1: Tests for the effects of time (T) and location (L) in a loglinear analysis of the subtidal transition data. Each box designates a particular model, its goodnessof-fit $G^{2}$ statistic, its degrees of freedom, and its AIC value. The top box gives the results for the null model (Fate is dependent only on State). The lower boxes represent models that include higher order interactions between Fate and $T, L$, or both. Terms added to each model along with the corresponding changes in $G^{2}\left(\Delta G^{2}\right)$ and degrees of freedom are shown along the arrows. 
the $l$ th matrix in $\mathcal{S}$. The element $s_{i j}^{(l)}$ of $\mathbf{S}^{(l)}$ gives the probability that a patch in state $j$ will be in state $i$ the following year, in quadrat $l$. The transition probabilities for $\mathbf{S}^{(l)}$ are estimated as

$$
s_{i j}^{(l)}=\frac{\sum_{k}^{T} n_{i j k l}}{\sum_{i}^{S} \sum_{k}^{T} n_{i j k l}}
$$

where $n_{i j k l}$ is the number of patches that change from state $j$ to state $i$ at time $k$ in location $l$.

Similarly, let $\mathcal{T}$ represent the set of time-varying transition matrices, and let $\mathbf{T}^{(k)}$ denote the $k$ th matrix in $\mathcal{T}$. The transition probabilities for $\mathbf{T}^{(k)}$ are estimated as

$$
\left.t_{i j}^{(k)}\right)=\frac{\sum_{l}^{L} n_{i j k l}}{\sum_{i}^{S} \sum_{l}^{L} n_{i j k l}}
$$

Finally, let $\mathbf{A}$ denote the homogeneous transition matrix. The transition probabilities for $\mathbf{A}$ are estimated as

$$
a_{i j}=\frac{\sum_{k}^{T} \sum_{l}^{L} n_{i j k l}}{\sum_{i}^{S} \sum_{k}^{T} \sum_{l}^{L} n_{i j k l}}
$$

\subsubsection{Equilibrium Predictions}

To examine what the effects of temporal and spatial variation are on equilibrium predictions, we compared:

- the equilibrium distribution that would result if each of the quadrat-specific matrices in the set $(\mathcal{S})$ were treated as a homogenous Markov chain, with the observed species distribution in each quadrat.

- the equilibrium distribution that would result if each of the time-specific matrices in the set $(\mathcal{T})$ were treated as a homogenous Markov chain, with the observed species distribution at each time.

- the equilibrium distribution for the homogenous Markov chain A with the observed species distribution averaged over time and space. 
Note that the equilibrium predictions of the different sets of Markov chains depend exclusively on the transition probabilities measured in the data set, which are independent of observed species abundances in the quadrats (see for example Horn 1975). Comparing predicted and observed abundances provides insights into how small-scale temporal and spatial variation in transition processes affect the ability of Markov chain models to predict community composition.

The equilibrium distribution of a Markov chain is calculated using eigenanalysis. Let $\mathbf{M}_{k}$ be the $k$ th matrix within the set of spatially-vary (temporally-varying) transition matrices. If $\mathbf{M}_{k}$ is primitive, its largest eigenvalue equals 1 . The corresponding eigenvector $\mathbf{z}_{k}$ (i.e. the dominant eigenvector) gives the equilibrium distribution of patch states, to which the community would converge if the transition probabilities specified by $\mathbf{M}_{k}$ remained constant.

If $\mathbf{u}_{l}$ is the dominant eigenvector of $\mathbf{S}^{(l)}$, then the mean equilibrium distribution for the set $\mathcal{S}$ is

$$
\overline{\mathbf{u}}=\frac{1}{L} \sum_{l=1}^{L} \mathbf{u}_{l}
$$

where $L=10$. Similarly, if $\mathbf{v}_{k}$ is the dominant eigenvector of $\mathbf{T}^{(k)}$, then the mean equilibrium distribution for the set $\mathcal{T}$ is

$$
\overline{\mathbf{v}}=\frac{1}{T} \sum_{k=1}^{T} \mathbf{v}_{k}
$$

where $T=8$. Finally, the equilibrium distribution for homogenous transition matrix is given by the dominant eigenvector of $\mathbf{A}$, which we shall denote as $\mathbf{w}$. To measure the variance in the equilibrium distributions for the sets $\mathcal{S}$ and $\mathcal{T}$, we calculated the variance in each element of $\mathbf{u}_{k}$ and $\mathbf{v}_{k}$.

We can quantify the abilifty of the by comparing The equilibrium predictions of the different sets of Markov chains depend exclusively on the transition probabilities measured in the data set and not on the initial abundances of species in the quadrats (see for example Horn 1975). 
Figure 5.2 shows comparisons of the equilibrium species distribution predicted by the Markov chains vs. relative species abundance within the quadrats. Fig. 5.2a compares the mean equilibrium distribution for the set of spatially-varying transition matrices $(\overline{\mathbf{u}})$ with the mean value of the average relative species abundance in each quadrat between 1986 and 1994. The agreement is excellent. Figure 5.2b compares predicted $\left(\mathbf{u}_{l} l=1, \ldots, L\right)$ and observed relative abundances, over all species and all quadrats. The correlation is $r_{p}=0.94, p<0.001$. Comparisons of the equilibrium distributions for the set of temporally-varying transition matrices (fig. 5.2c, d), and the homogenous transition matrix (fig. 5.2e, f) with observed species abundances yield similar results. This last result indicates that homogenous Markov chains can accurately predict community composition in the rocky subtidal zone. Thus while temporal and spatial variation is statistically significant, the effect on predicted abundances is biologically trivial.

\subsubsection{Community Dynamics}

Pooling transition data and eigenvector analyses provide information on long-term trends in species abundances, but tell us little about community dynamics. Here, we illustrate how temporal variation in transition probabilities affect community dynamics, using the set of time-varying transition matrices to model community dynamics as a non-homogenous Markov chain.

The general form for the non-homogenous Markov chain is given by

$$
\begin{aligned}
\mathbf{x}(t+1) & =\mathbf{A}_{t} \mathbf{x}(t) \\
& =\mathbf{A}_{t} \mathbf{A}_{t-1} \mathbf{A}_{t-2} \ldots \mathbf{A}_{1} \mathbf{x}(0)
\end{aligned}
$$

where $\mathbf{x}(t)$ is a column vector giving the probability distribution of patch states at time $t, \mathbf{x}(0)$ is some initial distribution of patch states, and the sequential product $\mathbf{A}_{1}, \mathbf{A}_{2}, \ldots, \mathbf{A}_{t}$ represents a single stochastic realization of the environment. 

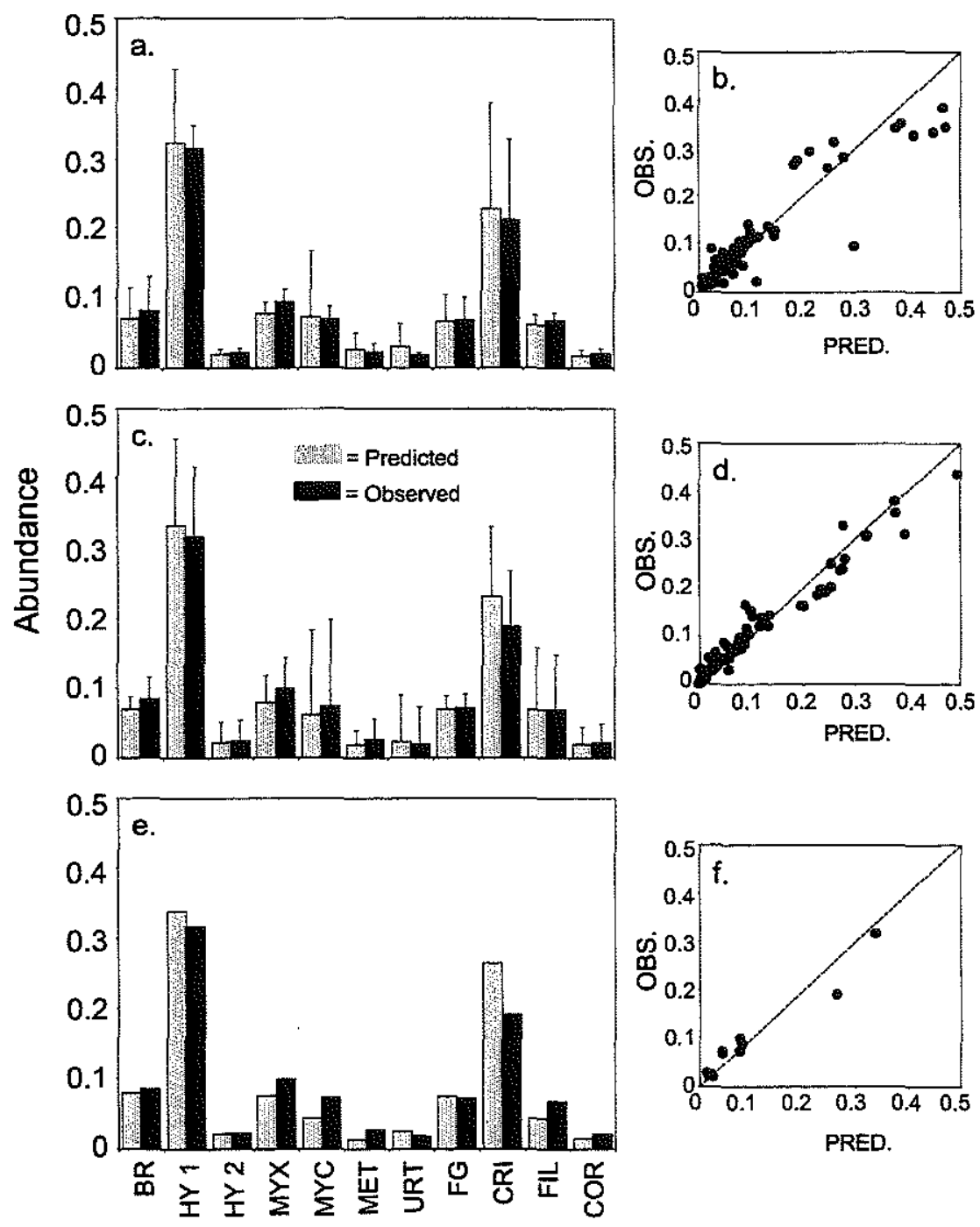

Figure 5.2: Predicted vs. observed species frequencies. Quadrat-specific transition matrices $\mathcal{S}: a$. Equilibrium distribution specified by the set of quadrat-specific transition matrices $\mathbf{u}$ vs. the mean observed species distribution in each quadrat, averaged over space (error bars represent $1 \mathrm{SD}$ ). $b$. Equilibrium distribution specified for each quadrat $\mathbf{u}_{k}(k=1, \ldots, L)$ as a function of the observed species distribution in each quadrat $\left(r_{p}=0.94, p<0.001\right)$. Time-specific transition matrices $T: c$. Equilibrium distribution specified by the set of time-specific transition matrices $\mathbf{v}$ vs. the mean observed species distribution in each year, averaged over time. $d$. Equilibrium distribution specified for each time interval $\mathbf{v}_{k}(k=1, \ldots, T)$ as a function of the observed species frequencies at each year $\left(r_{p}=0.98, p<0.001\right)$. Homogeneous transition matrix: $e$. and $f$. Equilibrium distribution specified by the dominant eigenvalue of $\mathbf{A}$ vs. the observed species distribution averaged over time and space $\left(r_{p}=0.99, p<0.001\right)$. 
The dynamics of the community depend on the sequential ordering of the matrices $\mathbf{A}_{t}$. If we assume that the matrices $\mathbf{A}_{t}(t=1, \ldots, \infty)$ are drawn from the set $\mathcal{T}$ then the sequence of transition matrices in equation 5.7 can be described by a columnstochastic transition matrix $\mathbf{P}$, whose $(i, j)$ entry is

$$
p_{i j}=P\left(\mathbf{A}_{t+1}=\mathbf{T}^{(i)} \mid\left(\mathbf{A}_{t}=\mathbf{T}^{(j)}\right)\right.
$$

(Caswell 2000).

We simulated community dynamics using for two different types of environmental variability to specify the sequence of matrices in equation 5.7 .

- Periodic environment; in which the sequence of transition matrices $\mathbf{T}^{(i)}$ was specified by the matrix

$$
\mathbf{P}=\left(\begin{array}{cccccc}
0 & 0 & 0 & \cdots & 0 & 1 \\
1 & 0 & 0 & \cdots & 0 & 0 \\
0 & 1 & 0 & \cdots & 0 & 0 \\
0 & 0 & 1 & \cdots & 0 & 0 \\
\vdots & \vdots & \vdots & \ddots & \vdots & \vdots \\
0 & 0 & 0 & \cdots & 1 & 0
\end{array}\right)
$$

- Stochastic environment; in which the sequence of transition matrices $\mathbf{T}^{(i)}$ were chosen independently and with equal probability; i.e. according to the matrix

$$
\mathbf{P}=\left(\begin{array}{cccc}
\frac{1}{8} & \frac{1}{8} & \cdots & \frac{1}{8} \\
\frac{1}{8} & \frac{1}{8} & \cdots & \frac{1}{8} \\
\vdots & \vdots & \ddots & \vdots \\
\frac{1}{8} & \frac{1}{8} & \cdots & \frac{1}{8}
\end{array}\right)
$$

Community dynamics specified by the non-homogeneous Markov chain for the 


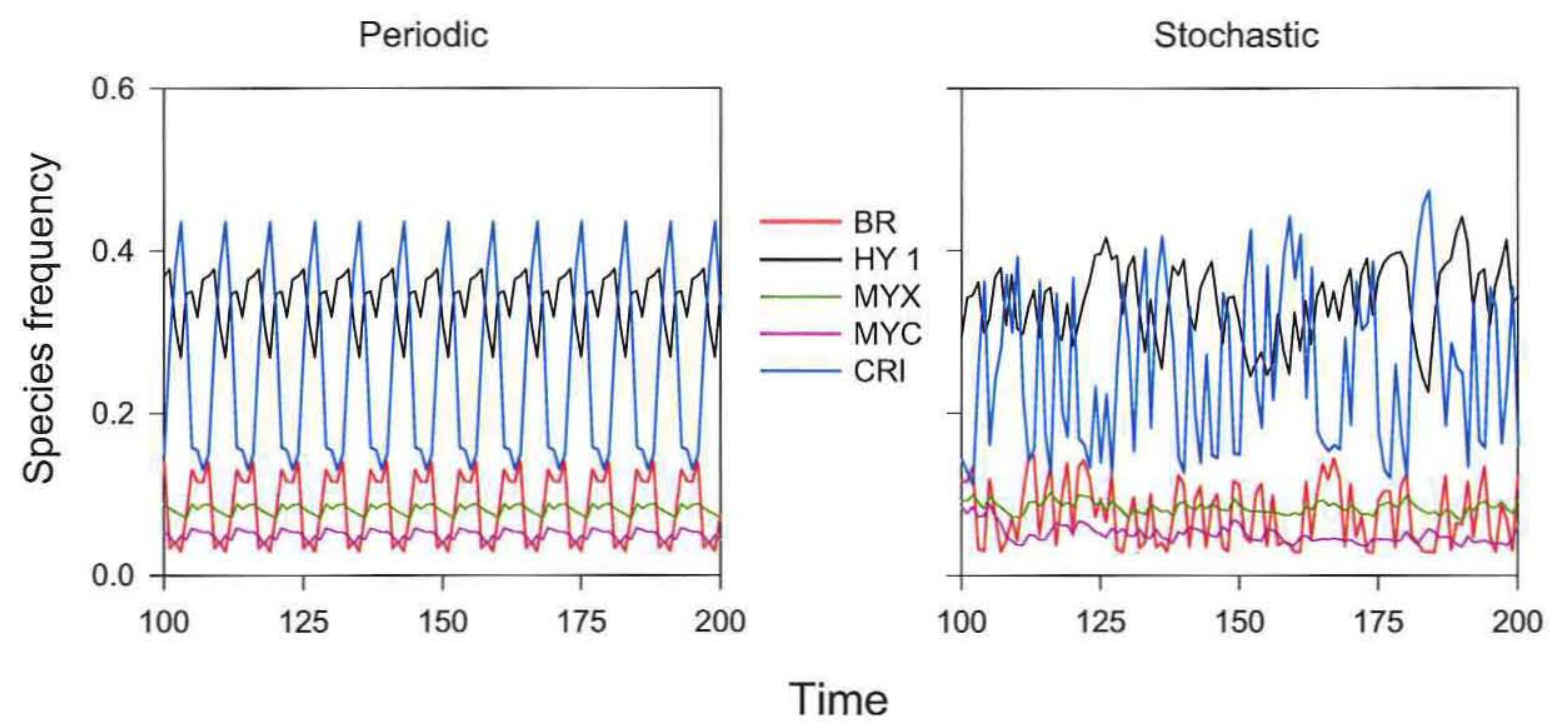

Figure 5.3: Simulated realizations of the non-homogeneous Markov chain showing the transient dynamics of Hymedesmia 1, Crisia, Myxilla, Mycale, and Bare Rock in a periodic and stochastic environment.

five most abundant states, Hymedesmia 1, Crisia, Myxilla, Mycale, and Bare Rock, are shown in figure 5.3. Simulations in both environments predict that species abundances are highly variable through time, with frequent switches in dominance between Hymedesmia 1 and Crisia (where dominance refers to the species with the greatest abundance in the community).

Figure 5.4 shows frequency distributions of dominance times for Hymedesmia 1 (fig. 5.4a) and Crisia (fig. 5.4b). Dominance time refers to a continuous length of time in which species $i(i=H Y 1$ or $C R I)$ is the most abundant species in the community. The median dominance time for Hymedesmia 1 is three years, with a range of 1 to 19 years. The median dominance time for Crisia is one year, with a range of 1 to 7 years.

Table 5.2 lists temporal correlations between the abundances of several subtidal species with Hymedesmia 1 and Crisia. The pattern of correlations indicates that the composition of the community changes depending on which species, Hymedesmia 1 


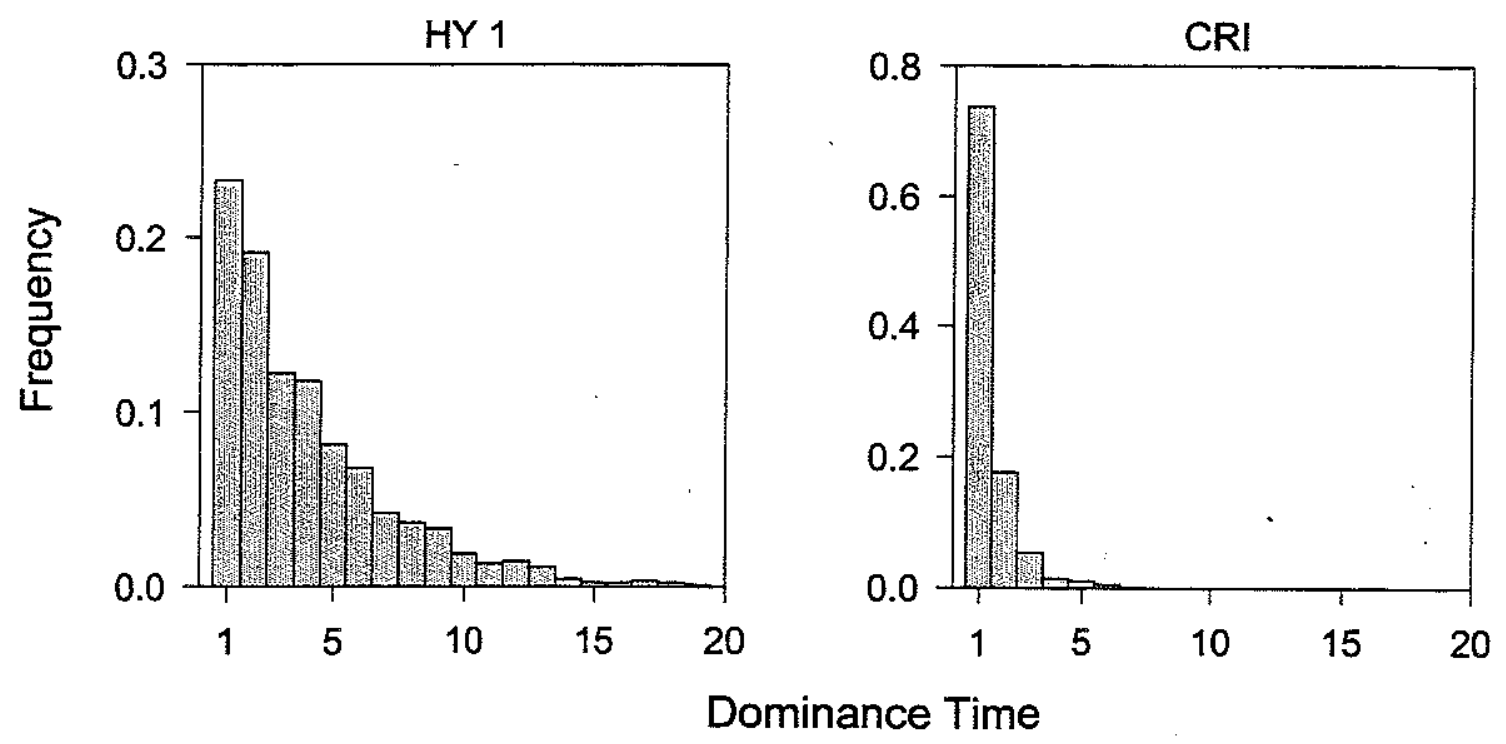

Figure 5.4: Frequency distribution of the dominance time of Hymedesmia 1 and Crisia. Results are based on 10,000 stochastic iterations of the non-homogeneous Markov chain using the stochastic environment.

\begin{tabular}{lcc}
\hline \hline & HY 1 & CRI \\
\hline BR & 0.256 & -0.880 \\
HY 1 & $\cdots$ & -0.557 \\
HY2 & 0.304 & $-0.110 *$ \\
MYX & $0.114 *$ & -0.499 \\
CRI & -0.557 & $\ldots$ \\
FG & 0.270 & -0.510 \\
COR & 0.237 & -0.713 \\
\hline \hline
\end{tabular}

Table 5.2: Product-moment correlation coefficients $r_{p}$ for the temporally-varying Markov chain. The second and third columns of the table show $r_{p}$ values for Hymedesmia 1 (HYM 1) and Crisia (CRI) vs. the states listed in the first column. Correlations are based on 10,000 iterations of the non-homogenous Markov chain in the stochastic environment. All correlations are significant at the $p<0.01$ level except where marked by $a *$, in which case $p<0.05$.

or Crisia, is most abundant. Since Hymedesmia 1 and Crisia oscillate out of phase with each other (Fig. 5.3), the correlation analysis suggests that species composition switches between two points in community phase space. 


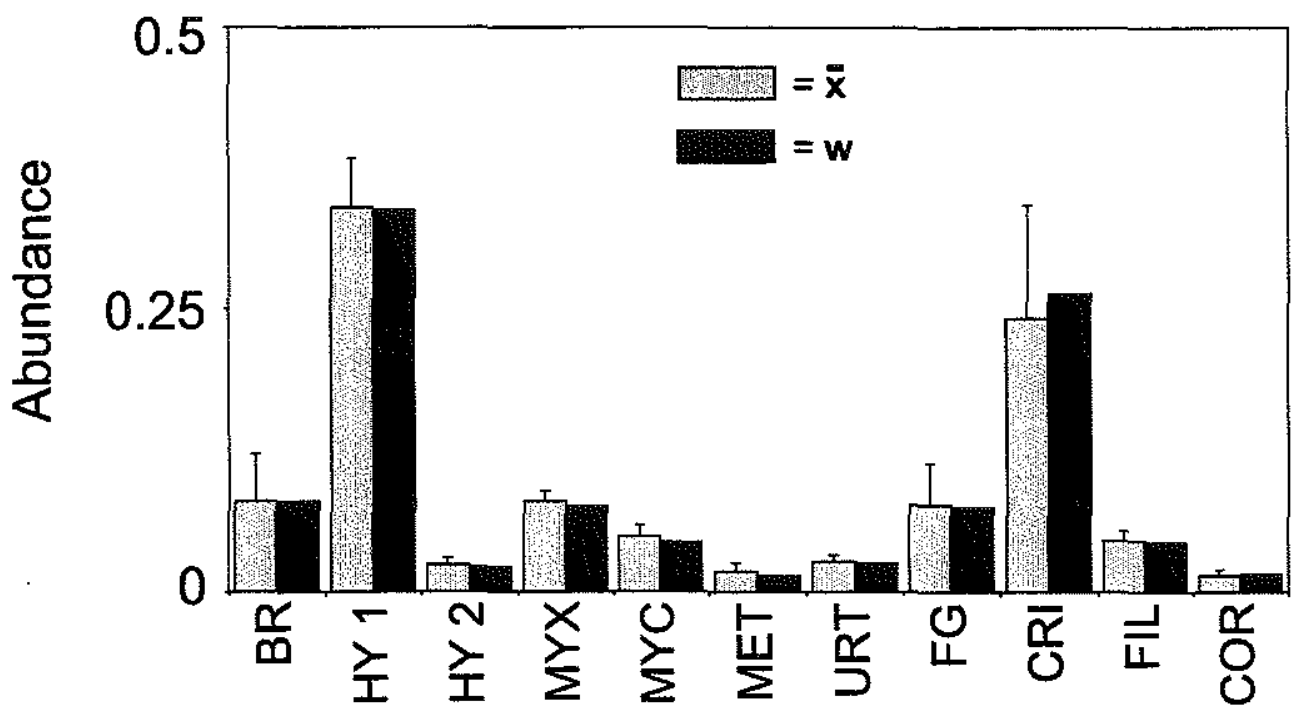

Figure 5.5: Comparison of the long-term temporal average of species abundance for the non-homogeneous Markov chain $(\overline{\mathbf{x}})$ and the equilibrium distribution (w predicted by the homogenous Markov chain. Averages for the non-homogeneous Markov chain are based on 10,000 iterations in the stochastic environment (the periodic environment gives similar results). Error bars represent $1 \mathrm{SD}$.

The long-term temporal average of the species composition for the non-homogeneous Markov chain can be calculated as

$$
\overline{\mathbf{x}}=\lim _{t \rightarrow \infty} \frac{1}{t} \sum_{k=0}^{t} \mathbf{x}_{k}
$$

where $\mathbf{x}_{k}$ is a vector whose $j$ th element is the abundance of species $j$ at time $k$. Figure 5.5 compares $\overline{\mathrm{x}}$ with the equilibrium distribution, $\mathbf{w}$, for the homogenous Markov chain. In general, there is no difference in the predicted mean species abundances between these methods of calculation.

The temporal and spatial variations in transition probabilities, as measured by log-linear analysis, reflect the highly dynamic nature of species succession in the subtidal community. The effects of this variation on community dynamics cannot be understood through eigenanalysis, but instead require stochastic realizations of time- 
and/or spatial-varying Markov chain to examine the behavior of the community. The average species composition of the community (over long time scales), however, can be obtained either from the dominant eigenvector of the homogeneous Markov chain A or by simulating the non-homogenous Markov chain specified by the matrices $\mathcal{T}$ and then applying equation 5.11 .

\subsection{Temporal and spatial variability in species dis- tributions}

We have shown that transition probabilities and community dynamics vary significantly in time and space, but what about the relative spatial and temporal distribution of individual species? To answer this question we employed a method for comparing stochastic matrices introduced by Cohen et al. (1998). The general idea is to construct a set of probability matrices $\mathcal{M}$, in which the $k$ th matrix of the set $\left(\mathbf{M}_{k}\right)$ describes the distribution of species $k$ among locations (time periods) at each time period (location). Variations in the spatial (temporal) distribution of species $k$ is assesses by measuring the mutual distance between the columns of $\mathbf{M}_{k}$.

Let $\mathbf{Q}$ be a 3 dimensional array, where the $(s, l, t)$ element of $\mathbf{Q}$ is the number of patches containing species $s$, at location $l$, at time $t$. For each species $s$, we define the $L \times T$ column stochastic matrix $\mathbf{A}_{s}$ with elements $\mathbf{A}_{s}(l, t)=\mathbf{Q}(s, l, t) /\left(\sum_{l} \mathbf{Q}(s, l, t)\right)$, and the $T \times L$ column stochastic matrix $\mathbf{B}_{s}$ with elements $\mathbf{B}_{s}(t, l)=\mathbf{Q}(s, l, t) /\left(\sum_{t} \mathbf{Q}(s, l, t)\right)$. Column $t$ of $\mathbf{A}_{s}$ is the probability distribution over locations of species $s$ at time $t$. If the columns of $\mathbf{A}_{s}$ are nearly identical then there is little temporal variation in the spatial distribution of species $s$ (i.e. relative abundances of species $s$ within the quadrats are similar over time). Column $l$ of $\mathbf{B}_{s}$ is the probability distribution over

time of the abundance of species $s$ at location $l$. If the columns of $\mathbf{B}_{s}$ are nearly identical then there is little spatial variation in the temporal distribution of species $s$ (i.e. relative abundances of species $s$ over time are similar between the quadrats). 
We can assess the mutual distance between columns in any probability matrix $\mathbf{M}$ using Dobrushin's coefficient of ergodicity

$$
\bar{\alpha}(\mathbf{M})=\frac{1}{2} \max _{j, k}\left\|m_{: j}-m_{: k}\right\|_{p}
$$

(Dobrushin 1956a,b), where $m_{: j}$ is the $j$ th column of $\mathbf{M}$ and $\|\mathbf{x}\|_{p}=\left(\sum|\mathbf{x}|^{p}\right)^{1 / p}$ is the $p$ norm $(p \geq 1)$ for the vector $\mathbf{x}$. Calculating $\bar{\alpha}\left(\mathbf{A}_{s}\right)(\mathrm{s}=1, \ldots, 12)$ gives a measure of how much the spatial distribution patterns of species $s$ varies in time. Calculating $\bar{\alpha}\left(\mathbf{B}_{s}\right)$ gives a measure of how much the temporal distribution patterns of species $s$ varies among locations.

Figure 5.6 shows $\bar{\alpha}\left(\mathbf{A}_{s}\right)$ and $\bar{\alpha}\left(\mathbf{B}_{s}\right)$ values for all 11 species states. The species with the greatest temporal variation in their spatial distribution patterns are Hymedesmia 2, Metridium, and Filograna (fig. 5.6a). The species with the greatest spatial variation in their temporal distribution patterns are Hymedesmia 2, Mycale, Metridium, Urticina, Crisia, Filograna and coralline algae (fig. 5.6b). Note that the spatial variation in the temporal distribution of Bare Rock is also relatively high. If we assume that the frequency of Bare Rock is correlated with levels of predation and on rock wall invertebrates, then this result suggest that the temporal frequency of predator attacks (and other forms of disturbance) is relatively variable in space. Finally, figure 5.6 shows that the relative variability in the abundance of Hymedesmia 1, Myxilla, and the functional group species (FG) are both temporally and spatially stable.

\subsection{Discussion}

To evaluate the significance of temporal and spatial heterogeneity in patch transition processes in a rocky subtidal community, we used log-linear analysis of the observed transition frequency tables. These models are the more powerful descendants of the original Anderson and Goodman (1957) test, which were introduced to test the time-homogeneity of Markov chains. While this test has been applied to matrix 

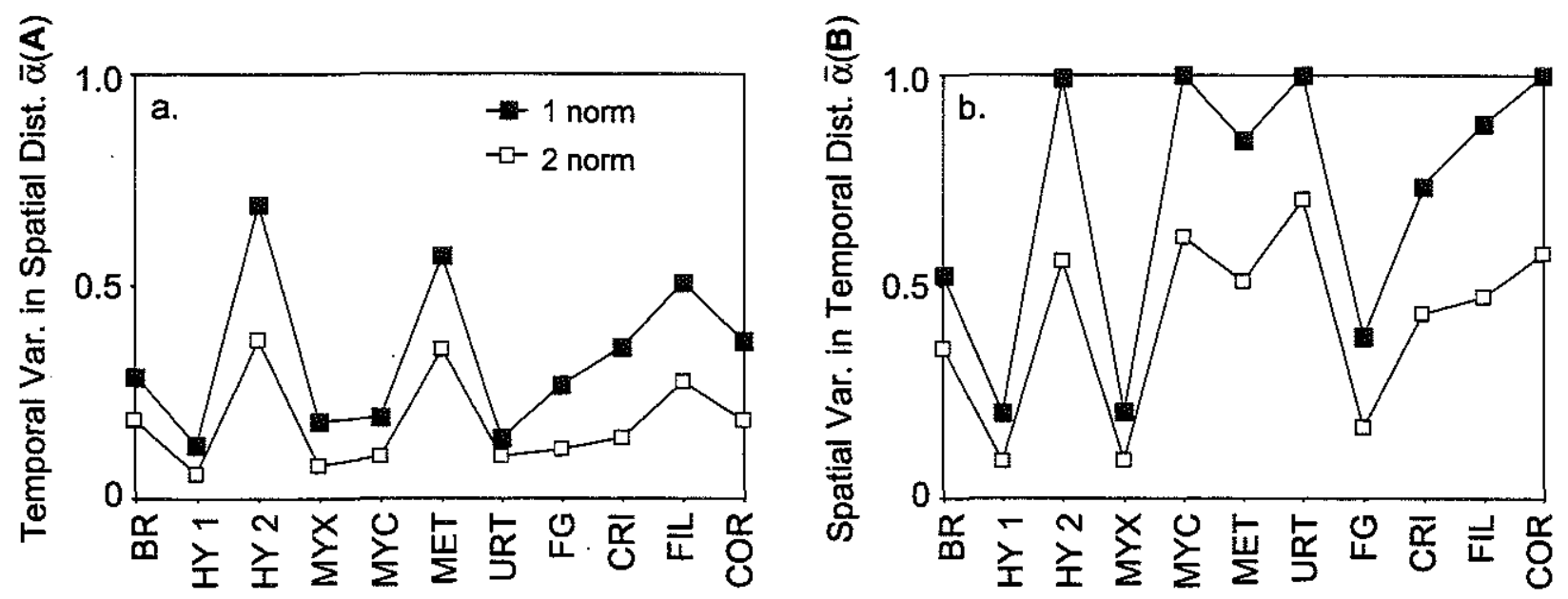

\section{Species}

Figure 5.6: Temporal and spatial variability of relative species abundances in the rocky subtidal community. Results are shown using the 1 norm and the 2 norm to calculate Dobrushin's coefficient of ergodicity (see text for details). a. Temporal variation in the spatial distribution of each species. A species with a low value of $\bar{\alpha}(\mathbf{A})$ has a spatial pattern of relative abundance that varies less in time than a species with a high value of $\bar{\alpha}(\mathbf{A})$. $b$. Spatial variation in the temporal distribution of each species. A species with a low value of $\bar{\alpha}(\mathbf{B})$ has a temporal pattern of relative abundance that varies less among quadrats than a species with a high value of $\bar{\alpha}(\mathbf{A})$. 
population models (Bierzychudek 1982; Cochran 1986) and community transition matrices (Tanner et al. 1994), the log-linear approach is more general and can be extended to more complex experimental designs (Caswell 2000). The Anderson and Goodman test is a likelihood ratio test for comparing the effects of a single factor (e.g. time) on transition probabilities. In essence, using it to investigate a more complex experimental design is analogous to using all possible pair wise t-tests in place of an ANOVA. While some authors continue to use the Anderson and Goodman test to develop community Markov chains, it is not an appropriate method for characterizing the effects of time and space on transition probabilities, and should be retired.

A log-linear analysis showed that patch transition probabilities varied significantly between locations and between years. Such variation can result from stochastic processes such as environmentally induced fluctuations in recruitment, or deterministic processes such as density- and spatial-dependent species interaction effects. The importance of including temporal and spatial variation in a model of the subtidal community, however, depends on the questions being asked. If we are interested in predicting long term trends in community composition, over large spatial areas, then a homogenous Markov chain (formed by pooling all the transition data across time and space) should provide adequate estimates of average species densities. On the other hand, if we want to understand the biological processes that drive transient dynamics in communities, then we need to develop Markov chains that incorporate processes affecting patch transition probabilities over smaller spatial and temporal scales.

In communities with many species it is important to quantify how the distribution of each species varies over time and space. The methods of Cohen et al. (1998) provide a simple but effective way of characterizing the spatial and temporal stability of individual species relative to other organisms in the community. For example in the subtidal community, the two dominant species, Hymedesmia 1 and Crisia, show somewhat different stability patterns. The distribution of Hymedesmia 1 is highly 
stable both temporally and spatially, suggesting that fluctuations in its abundance are highly synchronized across large spatial scales. The temporal distribution of Crisia, on the other hand, tends to vary a lot over small spatial scales, indicating that Crisia is more affected by changes in local conditions than Hymedesmia 1.

Figure 5.6 shows that there is more variability between quadrats in the temporal distribution of species, than there is over time in the spatial distribution of species. This result suggests location has a greater effect on species distribution than time and is consistent with the log-linear analysis, which shows a greater effect of space on transition probabilities (excluding $F T$ effects has a lower $A I C$ value than excluding $F L$, Fig. 1). Testing for differences in the variability of species distribution, however, is only a qualitative test, and is not as statistically rigorous as log-linear analysis. To put Cohen's approach into practice will require the development of statistical tests. Such tests can be developed using analytical methods, resampling techniques and randomization procedures (Cohen et. al. 1998).

One of our goals is to understand the relative extent to which deterministic forces are responsible for spatial and temporal fluctuations in the transition probabilities. To do this we are currently developing maximum likelihood methods to identify transitions whose values are dependent on species densities. We are using these methods to develop a nonlinear Markov chain model of the subtidal community in order to study long term community dynamics, look for multiple stable states and nonlinear dynamics, and examine whether species can be competitively excluded from the community (Hill, M., J.D. Witman and H. Caswell, in prep.). 


\subsection{References}

Akaike, H. 1973. in International Symposium on Information Theory, eds. B.N. Petran \& F. Csaki. Akademiai Kiado, Budapest, Hungary

Anderson, T.W. and L.A. Goodman. 1957. Statistical inference about Markov chains. Annals of Mathematical Statistics, 28:89-110.

Ayling, A.M. 1981. The role of biological disturbance in temporal subtidal encrusting communities. Ecology, 62:830-847.

Bierzychudek, P. 1982. The demography of Jack-in the pulpit, a forest perennial that changes sex. Ecological Monographs, 52:335-351.

Bishop, Y.M.M., S.E. Fienberg, and P.W. Holland. 1975. Discrete multivariate analysis. MIT Press, Cambridge.

Burnham, K.P. and D.R. Anderson. 1998. Model Selection and Inference: A Practical Information-Theoretical Approach. Springer-Verlag, New York, New York.

Caswell H. 1989. Matrix population models. Construction, analysis, and interpretation. Sinauer Associates Inc., Sunderland, MA.

Caswell H. 2000. Matrix population models. Construction, analysis, and interpretation. Sinauer Associates Inc., Sunderland, MA. 2nd Ed., in press.

Christensen, R. 1990. Log-Linear Models. Springer-Verlag, New York, New York.

Cochran, M.E. 1986. Consequences of pollination by chance in the pink lady's slipper, Cypripedium acaule. Ph.D. Dissertation, University of Tennessee, Knoxville.

Cohen J.E., J.H.B. Kemperman, and Gh. Zbăganu. 1998. Comparisons of Stochastic Matrices; with applications in Information Theory, Statistics, Economics, and Population Sciences. Birkhäuser, Boston, MA.

Dayton, P.K., G.A. Robilliard and R.T. Paine. 1970. Benthic faunal zonation as a 
result of anchor ice at McMurdo Sound, Antarctica. In Antarctic ecology. Martin Holdgate ed., Academic Press, London

Dobrushin, R.L. 1956a. Central limit theorem for nonstationary Markov chains, I. Theory of Probability and its Application, 1:65-80.

Dobrushin, R.L. 1956b. Central limit theorem for nonstationary Markov chains, II. Theory of Probability and its Application, 1:329-383.

Duggins, D.O. 1983. Starfish predation and the creation of mosaic patterns in a kelp dominated community. Ecology, 64:1610-1619.

Genovese, S.J. 1996. Regional and temporal variation in the ecology of an encrusting bryozoan in the Gulf of Maine. Ph.D. Dissertation, Northeastern University.

Genovese, S.J. and J.D. Witman. 1999. Interactive effects of flow speed and particle concentration on the growth rates of an active suspension feeder. Limnology and Oceanography, 44:1120-1131.

Lesser M.P., J.D. Witman, and K.P. Sebens. 1994. Effects of flow and seston availability on the scope for growth of benthic suspension feeding invertebrates form the Gulf of Maine. Biological Bulletin, 187:319-335.

Leicheter, J.J. and Witman, J.D. 1997. Water flow over subtidal rock walls: relationship to disturbance and growth rate of sessile suspension feeders in the Gulf of Maine. Journal of Experimental Marine Biology and Ecology, 209:293-307.

Osman R.W. 1977. The establishment and development of a marine epifaunal community. Ecological Monographs, 47:37-63.

Scheibling, R.E. and A.W. Hennigar 1997. Recurrent outbreaks of disease in sea urchins Strongylocentrotus droebachiensis in Nova Scotia: evidence for a link with large-scale meterologic and oceanographic events. Marine Ecology Progress Series, 152:155-165. 
Sebens K.P. 1985. The ecology of the rocky subtidal zone. American Scientist, 73:548-557.

Sebens K.P. 1986. Spatial relationships among encrusting marine organisms in the New England subtidal zone. Ecological Monographs, 56:73-96.

Silva, J.F., J. Raventos, and H. Caswell. 1990. Fire and fire exclusion effects on the growth and survival of two savanna grasses. Acta Oecologica, 11:783-800.

Smith, F. and J.D. Witman. 1999. Species diversity in subtidal landscapes: Maintenance by physical processes and larval recruitment. Ecology, 80:51-69.

Tanner J.E., T.P. Hughes and J.H. Connell. 1994. Species coexistence, keystone species, and succession: a sensitivity analysis. Ecology, 75:2204-2219.

Witman J.D. 1985. Refuges, biological disturbance, and rocky subtidal community structure in New England. Ecological Monographs, 55:421-445.

Witman J.D. 1996. Dynamics of Gulf of Maine benthic communities. pp 51-69 in D. Dow and E. Braasch (eds.). The health of the Gulf of Maine ecosystem: cumulative impacts of multiple stressors. RARGOM Report 96-1, Hanover, NH.

Witman J.D. 1987. Subtidal coexistence: storms, grazing mutualism, and the zonation of kelp and mussels. Ecological Monographs, 57:167-187.

Witman, J.D. and R.A. Cooper. 1983. Distribution and contrasting patterns of population structure in the brachiopod Terebratulina septentrionalis from two subtidal habitats. Journal of Experimental Marine Biology and Ecology, 73:57-79.

Witman, J.D. and K.P. Sebens. 1988. Benthic community structure at a subtidal rock pinnacle in the central Gulf of Maine. In I. Babb and M. DeLuca (eds.), Benthic Productivity and Marine resources of the Gulf of Maine. National Undersea Research Program report, 88-3:67-104.

Witman, J.D. and P.K. Dayton. 2000. Rocky subtidal communities. In Bertness, 
M.D. and M. Hay eds. Marine Benthic Ecology. Sinauer Press (in press)

Wootton J.T. 2000. Effects of extinction in complex ecosystems: predictions from Markov models. Ecology, in press. 


\section{Appendix}

In log-linear analysis the $\log$ of the cell frequencies in $\mathbf{N}\left(n_{i j k l}\right)$ is modeled as a linear function of the effects of $F, S, T, L$, and their interactions. The null hypothesis is one of conditional independence: given the initial state, the fate is independent of time and location. The resulting model for the null hypothesis is

$$
\begin{aligned}
\log n_{i j k l}= & u+u_{F(i)}+u_{S(j)}+u_{T(k)}+u_{L(l)} \\
& +u_{F S(i j)}+u_{S T(i k)}+u_{S L(j l)}+u_{T L(k l)} \\
& +u_{S T L(j k l)}
\end{aligned}
$$

where $u$ is the $\log$ of the total number of transitions in the table, $u_{S(j)}$ represents the effect that the $j$ th initial state has on the cell frequencies in $\mathbf{N}, u_{F S(i j)}$ represents the effect that the interaction of the $j$ th initial state and the $i$ th fate have on the cell frequencies in $\mathbf{N}$, etc.

To test the effect of location, we add the effect of time to the null model by including the terms $u_{F T(i k)}$ and $u_{F S T(i j k)}$

$$
\begin{aligned}
\log n_{i j k l}= & u+u_{F(i)}+u_{S(j)}+u_{T(k)}+u_{L(l)} \\
& +u_{F S(i j)}+u_{S T(i k)}+u_{S L(j l)}+u_{T L(k l)} \\
& +u_{F T(i k)}+u_{F S T(i j k)}+u_{S T L(j k l)}
\end{aligned}
$$

which gives the model FST, STL. To test the effect of time, we add the effect of location to the null model by including the terms $u_{F L(i l)}$ and $u_{F S L(i j l)}$

$$
\begin{aligned}
\log n_{i j k l}= & u+u_{F(i)}+u_{S(j)}+u_{T(k)}+u_{L(l)} \\
& +u_{F S(i j)}+u_{S T(i k)}+u_{S L(j l)}+u_{T L(k l)} \\
& +u_{F L(i l)}+u_{F S L(i j l)}+u_{S T L(j k l)}
\end{aligned}
$$


which gives the model FSL, STL. Finally, to test for the effects of time and location we use the model FST, FSL, STL

$$
\begin{aligned}
\log n_{i j k l}= & u+u_{F(i)}+u_{S(j)}+u_{T(k)}+u_{L(l)} \\
& +u_{F S(i j)}+u_{F T(i k)}+u_{F L(i l)}+u_{S T(j k)}+u_{S L(j l)}+u_{T L(k l)} \\
& +u_{F S T(i j k)}+u_{F S L(i j l)}+u_{S T L(j k l)}
\end{aligned}
$$

The parameters for all models are estimated by maximum likelihood methods. See Caswell (2000) for a general discussion of this methodology or Silva, Raventos and Caswell (1991) for an example in a demographic study. 


\section{Chapter 6}

\section{A Nonlinear Markov Chain of a Rocky Subtidal Community: Quantifying the effects of species interactions on community dynamics}

The community stagnates without the impulse of the individual. The impulse dies away without the sympathy of the community

-William James

\subsection{Introduction}

Nonlinear species interactions can have important consequences for the structure and dynamics of natural communities (Lawton 1992; Paine 1992; McCann et al. 1998). Numerous models have shown that nonlinearities in population growth rates and interspecific interactions can give rise to chaotic dynamics (e.g. Gilpin 1979; Hastings and Powell 1991; Kot et al 1992; Caswell and Neubert 1998), and that community stability is dependent on the number and strength of these interactions (May 1973; McCann et al. 1998).

In marine benthic communities, nonlinearities can arise from density-dependent 
effects on larval settlement (Planes et al. 1998;), post-settlement mortality (Weiss 1948; Connell 1985; Zajac et al 1989; McShane 1991), and competition for space between adults (Grant 1977; Denley and Underwood 1979; Sebens 1985,1986; Bertness 1989; Witman and Dayton 2000). Most empirical studies attempting to quantify the effects of species interactions on the dynamics of benthic systems typically involve only one or a few sessile species (e.g., Davis 1988, Raimondi 1990, Minchinton and Scheibling 1991). Paine (1992) proposed measuring per capita interaction strengths among species through a series of species removal experiments. As an alternative, we propose to estimate interaction strengths using Markov chain models.

Markov chains provide a statistical approach to modeling community dynamics in marine systems and have been used to characterize successional change in coral reef communities (Tanneret al. 1994, 1996), subtidal communities (Hill et al. 2000), and intertidal communities (Wootton 2000). A Markov chain describes a community as a landscape of patches, each of which is in one of a number of possible states. States are defined by the presence of an individual of a given species or species group (functional group). The model is based on a transition matrix $\mathbf{A}$, whose $(i, j)$ entry gives the probability that a patch in state $j$ changes to state $i$ in one time step. Most applications of Markov chains to marine and terrestrial communities have been limited to linear models, in which transition probabilities are assumed to remain constant over time (e.g. Waggoner and Stephens 1970, Horn 1975, Usher 1979, Acevedo 1982, Salia and Erxini 1987, Grant et. al. 1988, Isagi and Nakagoshi 1990, Masaki et al. 1992, Rego et al. 1993, Tanner et al. 1994,1996, Formacion and Salia 1994, Hill et al. 2000).

A Markov chain can incorporate effects of species interactions on patch succession by allowing transition probabilities to depend on species densities (Caswell and Cohen 1991a,b, 1995, Barradas and Cohen 1994, Barradas et al. 1996). If $\mathbf{x}(t)$ is a vector whose ith element gives the proportion of the landscape occupied by state $i$ at time 
$t$, then the dynamics of the community over time is given by

$$
\mathbf{x}(t+1)=\mathbf{A}_{\mathbf{x}} \mathbf{x}(t)
$$

where the subscript on $\mathbf{A}_{\mathbf{x}}$ means that the elements of the transition matrix, $a_{i j}\left(\mathbf{x}_{t}\right)$, are functions of the state densities at time $t$. These functions must take into account the requirement that $\mathbf{A}_{x}$ is always nonnegative (i.e. $a_{i j}\left(\mathbf{x}_{t}\right) \geq 0 ; \forall t \geq 0$ ) and that each column of $\mathbf{A}_{x}$ always sums to 1 ( $\mathbf{A}_{x}$ is column-stochastic).

Nonlinear Markov chain models for simple communities have been studied theoretically by Caswell and Cohen (1991a,b, 1993, 1995), and Barradas et al. (1996). Those models described various models for competition, succession, predator-mediated coexistence, and local and regional determination of species richness. However, we know of no attempt to apply this theory to data on real communities (Although Cornell and Karlson (1997) have invoked some of it to explain patterns of species diversity in coral reef communities).

In this paper we develop a nonlinear Markov chain of a rocky subtidal community to study the effects of species interactions on community dynamics. We derive maximum likelihood methods to estimate density-dependent transition probabilities from spatial time series data. The data for our model comes from permanent quadrats monitored over an 8-year period of epifaunal invertebrate communities living on subtidal rock walls in the Gulf of Maine. We study the dynamics of the model using numerical simulation, characterizing the behavior of the community starting from a large set of initial conditions. We also parameterize nonlinear Markov chains by calculating species densities at different spatial scales, to study the dependence of interactions strengths on neighborhood size. Finally, we use bifurcation analysis to characterize how changes in the strength of the interactions among species affect the temporal dynamics of the community. 


\begin{tabular}{ll}
\hline \hline Model States & Species Type \\
\hline Bare Rock & \\
Hymedesmia $1 \mathrm{sp}$. & Sponge \\
Hymedesmia $2 \mathrm{sp}$. & Sponge \\
Myxilla fimbriata & Sponge \\
Mycale lingua & Sponge \\
Metridium senile & Sea anemone \\
Urticina crassicomis & Sea anemone \\
Aplidium pallidum & Ascidian \\
Ascidia callosa & Ascidian \\
Parasmittina jeffreysi & Bryozoan \\
Idmidronea atlantica & Bryozoan \\
Crisia eburnea & Bryozoan \\
Filograna implexa & Polychaete \\
Spirorbis spirorbis & Polychaete \\
Coralline Algae & Encrusting algae \\
\hline \hline
\end{tabular}

Table 6.1: Invertebrate species identified in the photo quadrat data set. These species were originally used by Hill et al. (2000) to develop a linear Markov chain of a rocky subtidal community.

\subsection{Data collection}

The focus of our study is a vertical rock wall community located at approximately 30 meters depth on Ammen Rock Pinnacle in the Gulf of Maine (Witman and Sebens 1988; Leichter and Witman 1997). The data for our model were collected over an eight-year period (1986-1994). They consist of a series of photographs chronicling the spatial distribution of sessile species on the rock wall substrate through time. Ten replicate quadrats, positioned randomly along a 20 meter span of rock wall habitat were photographed at least yearly with a Nikonos V mounted on a quadrapod camera frame (as in Witman 1985; Cayer et al. 1999). Color prints were made of the high resolution color slides to identify the species of five major taxa of epifaunal invertebrates (sponges, sea anemones, ascidians, bryozoans, and polychaetes). A total of 14 species were recorded in the quadrats (Table 6.1).

This community has already been described by several authors (e.g., Osman 1977, 


\begin{tabular}{|l|c|}
\hline \hline Model States & State Codes \\
\hline Hymedesmia $1 \mathrm{sp}$. & HYM \\
\hline Myxilla fimbriata & MYX \\
\hline Mycale lingua & MYC \\
\hline $\begin{array}{l}\text { Metridium senile } \\
\text { Urticina crassicomis }\end{array}$ & SEA \\
\hline Crisia eburnea & CRI \\
\hline Filograna implexa & FIL \\
\hline $\begin{array}{l}\text { Aplidium pallidum } \\
\text { Ascidia callosa } \\
\begin{array}{l}\text { Parasmittina jeffreysi } \\
\text { Idmidronea atlantica } \\
\text { Spirorbis spirorbis }\end{array}\end{array}$ & FG \\
\hline Bare Rock & BR \\
\hline \hline
\end{tabular}

Table 6.2: Species groups used to develop the nonlinear Markov chain. Species groups are identified in the model using the state codes in the right-hand column of the table.

Ayling 1981, Russ 1982, Duggins 1983, Sebens 1985,1986; Witman 1985,1987,1996, Scheibling and Hennigar 1997, Witman and Dayton 2000) and modeled as a linear Markov chain by Hill et al. (2000a). In the linear model five species were combined into a single group (Aplidium, Ascidia, Parasmittina, Idmidronea, and Spirorbis) as they were shown to perform functionally similar roles in community dynamics (see Hill et al. 2000 for details). In this paper we reduced the state space further by eliminating two species (Hymedesmia 2 and coralline algae) from the data set (due to their low abundance) and combining the sea anemones, Metridium senile and Urticina crassicornis, into a single group. Thus the state space for the nonlinear Markov chain consists of 7 species states, plus a bare rock state representing empty patches (table $6.2)$.

Data for the analysis were obtained by superimposing a lattice of evenly spaced points over quadrat photographs and following state transitions at each point through time. Approximately 600 points (a single point every $1 \mathrm{~cm}$ ) were assayed per quadrat. We chose this scale because it was approximately equivalent to the size of the smallest 


\begin{tabular}{ccccccccc}
\hline \hline & HYM & MXY & MYC & SEA & CRI & FIL & FG & BR \\
\hline To: HYM & $a_{11}\left(\mathbf{x}_{t}\right)$ & $a_{12}\left(\mathbf{x}_{t}\right)$ & $a_{13}\left(\mathbf{x}_{t}\right)$ & $\cdots$ & $\cdots$ & $\cdots$ & $\cdots$ & $a_{1 S}\left(\mathbf{x}_{t}\right)$ \\
MXY & $a_{21}\left(\mathbf{x}_{t}\right)$ & $a_{22}\left(\mathbf{x}_{t}\right)$ & $a_{23}\left(\mathbf{x}_{t}\right)$ & $\cdots$ & $\cdots$ & $\cdots$ & $\cdots$ & $a_{2 S}\left(\mathbf{x}_{t}\right)$ \\
MYC & $a_{31}\left(\mathbf{x}_{t}\right)$ & $a_{32}\left(\mathbf{x}_{t}\right)$ & $a_{33}\left(\mathbf{x}_{t}\right)$ & $\cdots$ & $\cdots$ & $\cdots$ & $\cdots$ & $a_{3 S}\left(\mathbf{x}_{t}\right)$ \\
SEA & $\vdots$ & $\vdots$ & $\vdots$ & $\ddots$ & & & & $\vdots$ \\
CRI & $\vdots$ & $\vdots$ & $\vdots$ & & $\ddots$ & & & $\vdots$ \\
FIL & $\vdots$ & $\vdots$ & $\vdots$ & & & $\ddots$ & & $\vdots$ \\
FG & $\vdots$ & $\vdots$ & $\vdots$ & & & & $\ddots$ & $\vdots$ \\
BR & $a_{S 1}\left(\mathbf{x}_{t}\right)$ & $a_{S 2}\left(\mathbf{x}_{t}\right)$ & $a_{S 3}\left(\mathbf{x}_{t}\right)$ & $\cdots$ & $\cdots$ & $\cdots$ & $\cdots$ & $a_{S S}\left(\mathbf{x}_{t}\right)$ \\
\hline \hline
\end{tabular}

Table 6.3: Schematic representation of the transition matrix for the nonlinear Markov chain. The vectors $\mathbf{x}_{t}$ in the matrix indicate that the transition probabilities are functions of the states densities at time $t$.

organism in our data set. For simplicity we will refer to each point as a patch, where the size of a patch is taken to be $1 \mathrm{~cm}^{2}$. Since individuals of many of the subtidal species are capable of growing much larger than $1 \mathrm{~cm}^{2}$, a single individual can occupy more than one patch.

\subsection{A nonlinear Markov chain model}

A nonlinear Markov chain is based on a matrix of density-dependent transition probabilities. The matrix is arranged so that column $j$ represents transitions from state $j$ at time $t$ to states $i, i=1, \ldots, S$, at time $t+1$. The form of the transition matrix for the subtidal community is shown in table 6.3.

Estimating the dependence of transition probabilities on species densities requires a flexible functional form and a procedure to estimate the parameters in the function. Multiple logistic regression is a familiar method for describing a binary outcome as a function of a set of independent variables. Here, we use the generalization of logistic regression to polychotomous variables describing multiple outcomes (e.g. Cox 1970, Hosmer and Lemeshow 1989). 
Suppose there are multiple outcomes for some event with probabilities $q_{i}, i=$ $1, \ldots, S$, and associated with each outcome is a vector $\mathbf{z}$ of independent variables and $a$ vector of parameters $\mathbf{b}_{i}$. Polychotomous logistic regression defines a logit function for each of the possible outcomes. To ensure that the resulting set of probabilities sum to one, we write logit functions by specifying one of the outcomes as a reference value. If we let $q_{S}$ be the reference outcome, then the logit function for $q_{i}$ is

$$
\begin{aligned}
\ln \frac{q_{i}}{q_{S}} & =b_{0, i}+b_{1, i} z_{1}+\cdots=+b_{S, i} z_{S} \\
& =\left\langle\mathbf{b}_{i}, \mathbf{z}\right\rangle
\end{aligned}
$$

where the first element $\left(z_{0}\right)$ of $\mathbf{z}=1$ and $\left\langle\mathbf{b}_{i}, \mathbf{z}\right\rangle$ represents the scalar product of the two vectors. The parameter $b_{0}$ is a constant, which is unaffected by changes in the independent variables. Using the fact that $\sum_{i} q_{i}=1$, it follows that the probability of outcome $i$ is

$$
q_{i}= \begin{cases}\frac{\left.\exp \left(\left\langle\mathbf{b}_{i}, \mathbf{z}\right\rangle\right)\right)}{1+\sum_{j}^{S-1} \exp \left(\left\langle\mathbf{b}_{j}, \mathbf{z}\right\rangle\right)} & \text { if } i=1, \ldots, S-1 \\ \frac{1}{1+\sum_{j}^{S-1} \exp \left(\left\langle\mathbf{b}_{j}, \mathbf{z}\right\rangle\right)} & \text { if } i=S\end{cases}
$$

To develop a nonlinear Markov chain, we treat the probabilities of all transitions from state $j$ (i.e column $j$ of $\mathbf{A}_{x}$ ) as a polychotomous logistic regression problem. The vector of independent variables is $\mathbf{z}=[1, \mathbf{x}]^{T}$, where $\mathbf{x}$ is the vector of species densities and the probability $q_{i}$ is the matrix entry $a_{i j}(\mathbf{x})$. Since we are interested in the effects of species interactions on transition probabilities, we use the bare rock state, $\mathrm{BR}$, as the reference outcome.

Let $\mathbf{b}_{i}^{(j)}$ be the vector of parameters associated with the probability of changing form state $\mathrm{jth}$ to state $i$ in a single time step. The set of functions $a_{i j}(\mathbf{x})$ in the $j$ th 
column of $\mathbf{A}$ are given by

$$
a_{i j}(\mathbf{x})= \begin{cases}\frac{\exp \left(\left\langle\mathbf{b}_{i}^{(j)}, \mathbf{z}\right\rangle\right)}{1+\sum_{k}^{S-1} \exp \left(\left\langle\mathbf{b}_{k}^{(j)}, \mathbf{z}\right\rangle\right)} & \text { if } i<S \\ \frac{1}{1+\sum_{k}^{S-1} \exp \left(\left\langle\mathbf{b}_{k}^{(j)}, \mathbf{z}\right\rangle\right)} & \text { if } i=S\end{cases}
$$

Note that the logistic function can range from nearly linear (no effect of density) to having a sharp threshold in the density effects. It is also flexible enough to represent both positive and negative density effects. Positive effects might result from gregarious settlement or from local reproduction; negative effects from competitive interactions.

\subsection{Methods}

\subsubsection{Parameter estimation}

The transition probabilities are estimated from data on the number of patches that change from state $j$ at time $t$ to state $i$ at time $t+1$, and the densities of the various states in each quadrat at time $t$. The density of state $i$ within a quadrat is the number of patches occupied by state $i$ divided by the total number of quadrat patches.

Let $\mathbf{B}^{(j)}$ be a matrix associated with the jth column of $\mathbf{A}$, whose ith row (denoted $\left.\mathbf{B}_{i}^{(j)}\right)$ is the vector $\mathbf{b}_{i}^{(j)}$. The elements of $\mathbf{B}^{(j)}$ are estimated by maximum likelihood methods. The likelihood function is

$$
\begin{aligned}
L\left(\mathbf{B}^{(j)} \mid \text { Data }\right)=\prod_{t=1}^{T} \prod_{l=1}^{L}\{ & \left(\prod_{i=1}^{S-1}\left[\frac{\exp \left(\left\langle\mathbf{B}_{i}^{(j)}, \mathbf{z}_{l t}\right\rangle\right)}{1+\sum_{k}^{S-1} \exp \left(\left\langle\mathbf{B}_{k}^{(j)}, \mathbf{z}_{l t}\right\rangle\right)}\right]^{N_{i, l, t}}\right) \\
& \left.\times\left(\frac{1}{1+\sum_{k}^{S-1} \exp \left(\left\langle\mathbf{B}_{k}^{(j)}, \mathbf{z}_{l t}\right\rangle\right)}\right)^{N_{S, l, t}}\right\}
\end{aligned}
$$

where $T$ is the number of time intervals, $\mathrm{L}$ is the number of quadrats, $\mathbf{z}_{l t}$ is a vector of 
species densities in quadrat $l$ at time $t$, and $N_{i, l, t}$ is the number of patches that change from state $j$ to state $i$ in quadrat $l$ within the interval $(t, t+1)$. This function insures that the probabilities in each column of $\mathbf{A}_{\mathbf{x}}$ always sum to one, and all possible pairs of probabilities have a logistic relationship (Cox 1970).

The log-likelihood is

$$
\begin{array}{r}
\ln L\left(\mathbf{B}^{(j)} \mid \text { Data }\right)=\sum_{t=1}^{T} \sum_{l=1}^{L} N_{1, l, t}\left(\mathbf{B}_{1}^{(j)}-\Phi_{l t}\right)+\cdots \\
+N_{(S-1), l, t}\left(\mathbf{B}_{(S-1)}^{(j)}-\Phi_{l t}\right)-N_{S, l, t} \Phi_{l t}
\end{array}
$$

where $\Phi_{l t}=\ln \left\{1+\sum_{k} \exp \left(\left\langle\mathbf{B}_{k}^{(j)} \mathbf{z}_{l t}\right\rangle\right)\right\}$. The values of the coefficients in $\mathbf{B}^{(j)}$ that maximize (6.6), are the maximum likelihood estimates of the model parameters in column $j$. This process is repeated for each column of $\mathbf{A}_{\mathbf{x}}$.

\subsubsection{Set of candidate models}

The set of models we fit to the data represent different hypotheses regarding the number of species interactions affecting the replacement of species $j$ by species $i$. These include a model with no species interactions $\left(M_{0}\right)$, plus a set of models in which each $a_{i j}(\mathbf{x})$ in column $j$ is a function of the density of a single species $\left(M_{1}\right)$, of two species $\left(M_{2}\right)$, of three species $\left(M_{3}\right)$, etc. The number of parameters estimated for each column of $\mathbf{A}_{\mathbf{x}}$ depends on which model we are fitting to the data (table 6.4). The maximum number of parameters is $(S+1) \times(S-1)$. This corresponds to the situation in which each $a_{i j}(\mathbf{x})$ in column $j$ is a function of the densities of all the species in the community (model $M_{8}$ ).

For a given model, $M_{h} h=0, \ldots, 8$, we used the MATLAB routine fminu to find parameter values that maximized the likelihood function (Eq. 6.6). To determine which model best approximates the mechanisms generating the transition data we used the Akaike Information Criteria (AIC) (Akaike 1973; Burham and Anderson 1998). If $L_{h}$ is the likelihood of model $M_{h}$ and $p_{h}$ is the number of parameters in 


\begin{tabular}{lll}
\hline \hline Model Name & No. of interactions & No. Parameters \\
\hline$M_{0}$ & 0 & $S-1$ \\
$M_{1}$ & 1 & $2(S-1)$ \\
$M_{2}$ & 2 & $2(S-1)$ \\
$\vdots$ & $\vdots$ & $\vdots$ \\
$M_{S}$ & $\mathrm{~S}$ & $(S+1)(S-1)$ \\
\hline \hline
\end{tabular}

Table 6.4: The models fitted to the subtidal data, with the number of species interactions affecting each entry $a_{i j}, i=1, \ldots, S$, in the $j$ th column and the number of parameters estimated for each column of $\mathbf{A}_{\mathbf{x}}$. The variable $S$ is the number of states in the Markov chain $(S=8)$.

model $M_{h}$, then the AIC for $M_{h}$ is

$$
A I C=-2 \ln L_{h}+2 p_{h}
$$

The model that minimizes AIC is considered the most parsimonious, best-fitting model (Akaike 1973). Because models are fit separately to each column, it is possible to have different best fitting models for different columns of $\mathbf{A}_{\mathbf{x}}$.

For each model $M_{h}, h=0, \ldots, 8$, we assume that the set of the probability functions $a_{i j}(\mathbf{x})$ in column $j$ depend on the densities of exactly $h$ species (table 6.4). Each $a_{i j}(\mathbf{x})$, however, can depend on a different set of species (as long as the set contains $h$ species). This means that the total number of possible models for each column is

$$
\sum_{n=0}^{S}\left(\frac{S !}{n !(S-n) !}\right)^{S}
$$

and for the entire transition matrix is

$$
\left(\sum_{n=0}^{S}\left(\frac{S !}{n !(S-n) !}\right)^{S}\right)^{S}
$$

Thus for a community with 8 states $(S=8)$ the total number of possible nonlinear Markov chains is approximately $10^{199}$. Obviously, this is an incredible large model 
space to search for the best-fitting nonlinear Markov Chain. Not surprisingly, trying to find the best-fitting model using polychotomous logistic regression methods alone takes an unreasonably long time.

\subsubsection{Model Fitting using a Two Step Method}

To more efficiently search this model space, we developed nonlinear Markov chains using the following two-step process.

1. We treat each $a_{i j}$ as a binary logistic regression problem to rank the strength of the effect each species has on the replacement of species $j$ by species $i$.

2. We then use the species rankings obtained in step 1 to specify a set of polychotomous logistic models for each column of $\mathbf{A}_{\mathbf{x}}$.

We can write a binary logistic model for each $a_{i j}$, by assuming there are two outcomes for a patch in state $j$; it either becomes occupied by state $i$ at time $t+1$, or it does not. The logit function for $a_{i j}$ is

$$
\ln \frac{a_{i j}}{1-a_{i j}}=\langle\mathbf{c}, \mathbf{y}\rangle
$$

where $\mathbf{c}$ is a vector of parameters and $\mathbf{y}=[1, \mathbf{x}]$ is a vector of species densities. Solving (6.10) for $a_{i j}$ yields

$$
a_{i j}=\frac{e^{\langle\mathbf{c}, \mathbf{y}\rangle}}{1+e^{\langle\mathbf{c}, \mathbf{y}\rangle}}
$$

To rank the species according to their effect on $a_{i j}$, we first assume that $a_{i j}$ depends on the density of a single species. For each species $k(k=1, \ldots, S)$ we maximize the likelihood formula

$$
\ln L(\mathbf{c} \mid \text { Data })=\sum_{l} \sum_{t} n(i, l, t)\left(\left\langle\mathbf{c}, \mathbf{y}_{l t}\right\rangle-\ln \left(1+e^{\left\langle\mathbf{c}, \mathbf{y}_{l t}\right\rangle}\right)\right.
$$


where $\mathbf{y}_{l t}$ are the densities of species $k$ in quadrat $l$ at time $t$ and $n(i, l, t)$ is the number of patches that change from state $j$ to state $i$ in quadrat $l$ within the time interval $(t, t+1)$. The species that yields the largest likelihood value $(\ln L)$ is ranked as having the strongest effect on the replacement of species $j$ by species $i$. We denote this species as $x^{(1)}$.

To identify the seconded ranked species we repeated this procedure, assuming that $a_{i j}$ depends on the density of $x^{(1)}$ and a second species $m\left(m \neq x^{(1)}\right)$. The species $m$ that yields the largest likelihood value (in combination with species $x^{(1)}$ ) is ranked as having the second strongest effect on the replacement of species $j$ by species $i$. This process is continued until all species have been ranked according to their effect on $a_{i j}(\mathbf{x})$. Note that estimations of the parameter values $\mathbf{c}$ are important for determining the best ranking of species, however, these values are not used to parameterize the nonlinear Markov chain.

Once the species ranking are specified for each $a_{i j}$, we write the transition probabilities in each column of $\mathbf{A}_{x}$ as a set of polychotomous logistic functions (Eq. 6.4). To fit a specific model $M_{h}$ to column $j$, we create a set of density matrices in the form

$$
\mathbf{z}_{l t}=\left(\begin{array}{cccc}
1 & 1 & \cdots & 1 \\
x_{1}^{(1)} & x_{2}^{(1)} & \cdots & x_{S-1}^{(1)} \\
\vdots & \vdots & \ddots & \vdots \\
x_{1}^{(h)} & x_{2}^{(h)} & \cdots & x_{S-1}^{(h)}
\end{array}\right)
$$

where $x_{i}^{(k)}$ is the density of the species with the $k$ th strongest effect on the replacement of species $j$ by species $i$ (as specified by the binary logistic analysis). For example, to fit the model $M_{2}$ we substitute the set of matrices

$$
\mathbf{z}_{l t}=\left(\begin{array}{cccc}
1 & 1 & \cdots & 1 \\
x_{1}^{(1)} & x_{2}^{(1)} & \cdots & x_{S-1}^{(1)} \\
x_{1}^{(2)} & x_{2}^{(2)} & \cdots & x_{S-1}^{(2)}
\end{array}\right)
$$


into the polychotomous likelihood function (Eq. 6.6).

\subsubsection{Best fitting model}

Table 6.5 shows log-likelihood and relative $\triangle A I C$ value $(\triangle A I C=A I C$ value relative to the best model) for the models $M_{h}, h=0, \ldots, 8$, for each column of $\mathbf{A}_{\mathbf{x}}$ (HYM, MYX, MYC, etc.). The best fitting model for each column has a $\triangle A I C$ value equal to 0.0. In general, the best fitting models include the densities of all but one or two of the species in the community. For example, the best fitting model for the set of functions $a_{1, i}(\mathbf{x})$ describing the replacement of Hymedesmia by species $i(i=1, \ldots, 8)$ include the densities of seven species.

Table 6.5 shows that the best nonlinear Markov chain is comprised of a transition

matrix $\mathbf{A}_{\mathbf{x}}$ whose first column consists of a set of probability functions specified by model $M_{7}$, whose second column consists of a set of probability functions specified by model $M_{6}$ model, etc. This transition matrix can be represented using the symbol $M_{76777766}$, where the first entry in the subscript gives the number of species interactions for column one, the second entry gives the number of species interactions for column two, and so on. The rank ordering of species interaction strengths for each of the probability functions $a_{i j}(\mathbf{x})$ is shown in the appendix.

\subsection{Results: Nonlinear Dynamics of the Subtidal Community}

\subsubsection{Numerical simulations}

The analysis of the nonlinear Markov Chain $M_{76777766}$ depends on numerical simulation, as there is no hope of finding analytical solutions for a model of this complexity. Numerical analysis were carried out by specifying an initial probability vector $\mathbf{x}(0)$ (where $\mathbf{x}(0)$ is an $8 \times 1$ non-negative column vector whose elements sum to 1 ) and 


\begin{tabular}{lccccccccc}
\hline \hline & \multicolumn{1}{c}{ HYM } & \multicolumn{2}{c}{ MYX } & \multicolumn{2}{c}{ MYC } & \multicolumn{2}{c}{ SEA } \\
Model & $\mathrm{n}$ & $\log L$ & $\Delta A I C$ & $\log L$ & $\Delta A I C$ & $\log L$ & $\Delta A I C$ & $\log L$ & $\Delta A I C$ \\
\hline$M_{0}$ & 7 & -8739.9 & 597.4 & -3213.3 & 273.9 & -1457.6 & 407.9 & -1681.9 & 517.4 \\
$M_{1}$ & 14 & -8565.5 & 262.6 & -3125.7 & 105.3 & -1361.2 & 229.1 & -1649.8 & 467.1 \\
$M_{2}$ & 21 & -8469.4 & 84.5 & -3084.2 & 43.5 & -1287.2 & 95.1 & -1515.5 & 4212.6 \\
$M_{3}$ & 28 & -8438.5 & 36.7 & -3059.9 & 9.0 & -1253.1 & 41.0 & -1438.4 & 72.4 \\
$M_{4}$ & 35 & -8428.4 & 30.3 & -3051.7 & 6.6 & -1237.5 & 23.8 & -1413.0 & 35.4 \\
$M_{5}$ & 42 & -8419.2 & 26.0 & -3046.2 & 9.6 & -1224.4 & 11.6 & -1395.5 & 14.5 \\
$M_{6}$ & 49 & -8411.3 & 24.2 & -3034.4 & $\mathbf{0 . 0}$ & -1217.0 & 10.7 & -1385.8 & 9.0 \\
$M_{7}$ & 56 & -8392.2 & $\mathbf{0 . 0}$ & -3031.0 & 7.2 & -1204.6 & $\mathbf{0 . 0}$ & -1374.3 & $\mathbf{0 . 0}$ \\
$M_{8}$ & 63 & -8391.3 & 12.2 & -3029.1 & 10.1 & -1202.8 & 10.4 & -1374.2 & 13.9 \\
\hline \hline
\end{tabular}

\begin{tabular}{lccccccccc}
\hline \hline & \multicolumn{1}{c}{ CRI } & \multicolumn{2}{c}{ FIL } & \multicolumn{2}{c}{$\overline{\mathrm{FG}}$} & \multicolumn{2}{c}{$\mathrm{BR}$} \\
Model & $\mathrm{n}$ & $\log L$ & $\Delta A I C$ & $\log L$ & $\Delta A I C$ & $\log L$ & $\Delta A I C$ & $\log L$ & $\Delta A I C$ \\
\hline$M_{0}$ & 7 & -7862.4 & 913.5 & -2456.3 & 347.3 & -4435.2 & 578.1 & -5432.2 & 817.4 \\
$M_{1}$ & 14 & -7559.2 & 321.0 & -2323.6 & 95.9 & -4239.7 & 196.1 & -5240.0 & 442.9 \\
$M_{2}$ & 21 & -7443.8 & 104.4 & -2303.9 & 70.6 & -4169.4 & 74.5 & -5069.7 & 120.6 \\
$M_{3}$ & 28 & -7418.5 & 67.6 & -2269.9 & 16.4 & -4145.5 & 40.7 & -5038.8 & 72.7 \\
$M_{4}$ & 35 & -7393.4 & 31.6 & -2260.0 & 10.6 & -4128.0 & 19.6 & -5015.2 & 39.5 \\
$M_{5}$ & 42 & -7378.5 & 15.8 & -2252.1 & 8.8 & -4117.9 & 13.4 & -5003.3 & 29.7 \\
$M_{6}$ & 49 & -7371.4 & 15.6 & -2244.2 & 7.0 & -4104.2 & $\mathbf{0 . 0}$ & -4981.5 & $\mathbf{0 . 0}$ \\
$M_{7}$ & 56 & -7356.6 & $\mathbf{0 . 0}$ & -2233.7 & $\mathbf{0 . 0}$ & -4099.7 & 4.9 & -4976.7 & 4.5 \\
$M_{8}$ & 63 & -7355.5 & 11.7 & -2233.6 & 13.9 & -4098.6 & 16.7 & -4975.4 & 15.9 \\
\hline \hline
\end{tabular}

Table 6.5: The models fitted to the photo quadrat data, with the number of parameters $(n)$ for each column, the $\log$ likelihood $(\log L)$, and the $A I C$ value relative to the best model $(\triangle A I C)$.

then iterating the model according to equation (6.1). At each iteration the value of the elements of $\mathbf{A}_{\mathbf{x}}$ were calculated as a function of $\mathbf{x}(t)$ using formula (6.4).

The nonlinear Markov chain has several classes of potential behavior

- Convergence to a stable equilibrium distribution $\hat{\mathbf{x}}$

- Convergence to multiple stable equilibrium distributions $\hat{\mathbf{x}}_{i}$

- Periodic, quasiperiodic, or chaotic dynamics

To examine all possible behaviors of the model we ran 10,000 simulations for 500 time steps. Each simulation was initialized by randomly choosing a probability vector $\mathbf{x}_{0}$ 
from a uniform distribution on the $S-1$ simplex.

\subsubsection{Equilibrium dynamics}

Simulations of the nonlinear Markov chain revealed three coexisting attractors; a stable 2-cycle and two stable equilibria (Fig. 6.1). The 2-cycle has the largest basin of attraction, with convergence to the 2-cycle attractor occurred in $57.2 \%$ of the simulations (Fig. 6.1a). Species densities on this attractor are almost completely dominated by Mycale, which occupies $89.1 \%$ of the patches (Fig. 6.1b). The functional group (FG) has the second highest density, with an average patch occupancy of $7.1 \%$. Three of the species states, Filograna, Crisia and the sea anemones, are almost completely eliminated from the community.

Initial conditions within the second largest basin of attraction converge monotonically to a stable equilibrium (occurring in $34.2 \%$ of the simulations) (Fig. 6.1c,d). Species densities on this attractor are dominated by Crisia and Hymedesmia, which occupy $55.1 \%$ and $30.0 \%$ of the substrate, respectively. We refer to this equilibrium distribution as the CRI-HYM equilibrium.

Initial conditions within the smallest basin of attraction converge slowly to a stable equilibrium via a damped 2-cycle oscillation (occurring in $8.6 \%$ of the simulations) (Fig. 6.1e,f). This attractor is dominated by the sea anemones, which occupies $49.6 \%$ of the substrate at equilibrium (SEA equilibrium). The remaining proportion of the substrate is almost completely unoccupied $(B R=49.0 \%)$, with no other species having densities greater than $0.5 \%$.

While the 2-cycle has the largest basin of attraction, the species frequencies predicted by the CRI-HYM equilibrium are much more similar to observed frequencies in the subtidal quadrats. Figure 6.2 shows a comparison of the CRI-HYM equilibrium species frequencies with the mean observed frequencies. The mean observed 
Stable 2-cycle
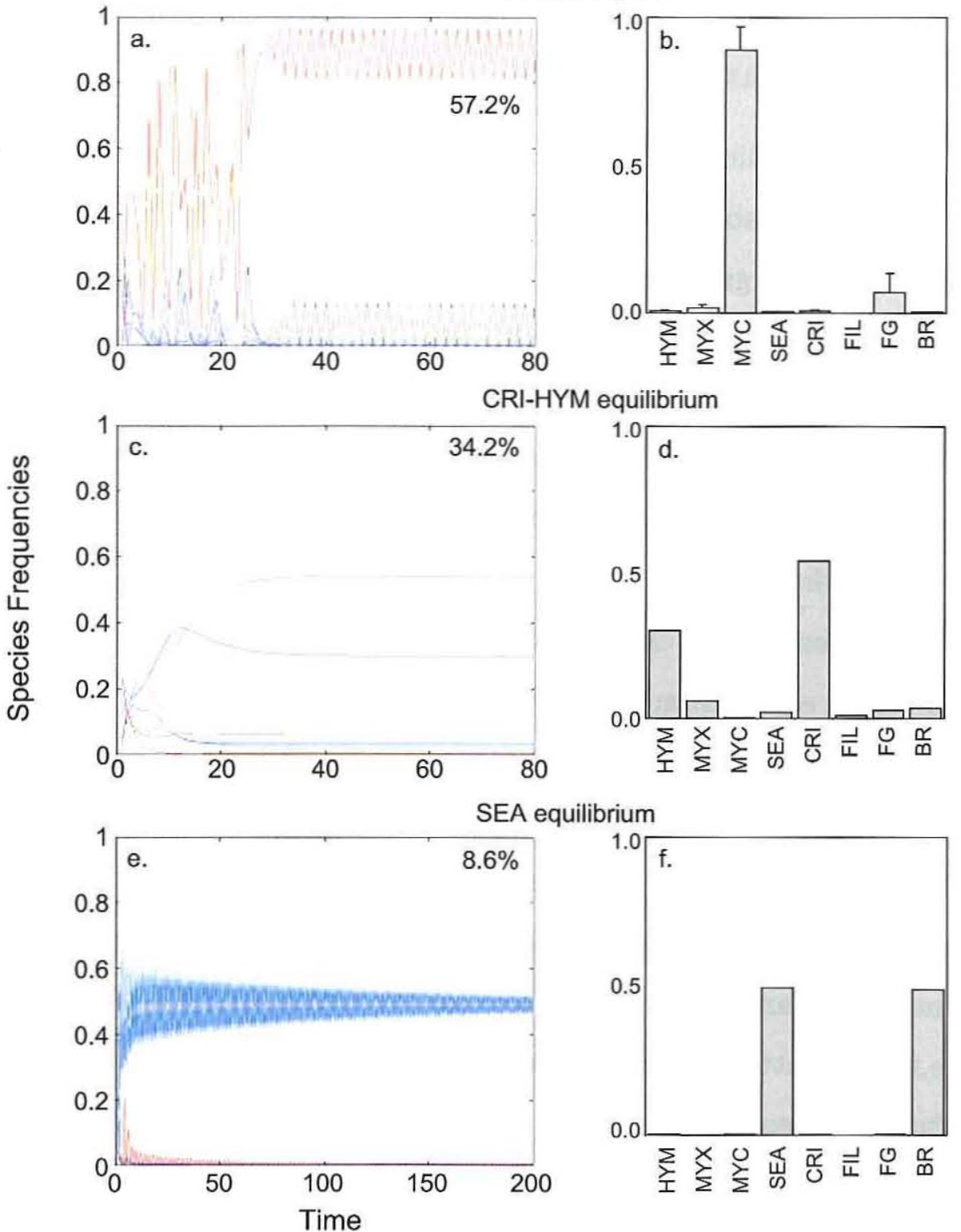

Figure 6.1: Coexisting attractors for the best fitting nonlinear Markov chain, in which parameters were estimated based on species densities within quadrats. Time series for the attractors are shown in $a, c$, and $e$, along with the proportion of times the model converged to each attractor starting from 10,000 random initial conditions. Histograms of the equilibrium species frequencies for each attractor are shown in $b$, $d$, and $f$. 


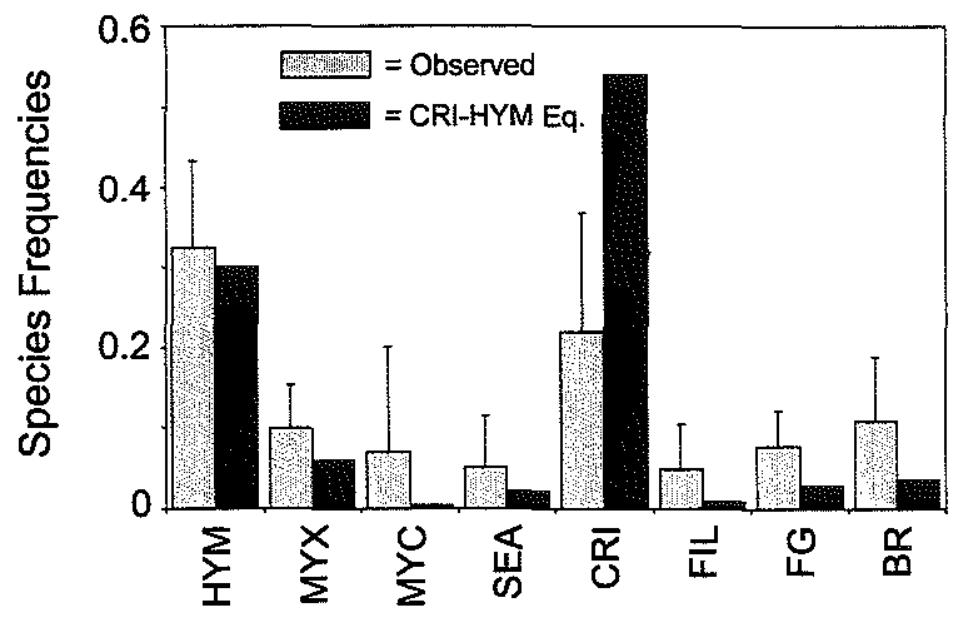

Figure 6.2: Mean abundance of subtidal species among quadrats over time (Observed) vs. CRI-HYM equilibrium distribution. Error bars represent $1 \mathrm{SD}$. The predicted $C R I-H Y M$ equilibrium abundances of all species except Crisia fall within 1 standard error of observed abundances.

frequency of species $i$ was calculated as

$$
\hat{O}_{i}=\frac{1}{L T} \sum_{l=1}^{L} \sum_{t=1}^{T} \frac{n_{i, l, t}}{\sum_{j} n_{j, l, t}}
$$

where $n_{i, l, t}$ is the number of patches containing species $i$ in quadrat $l$ at time $t$.

\subsubsection{Convergence times}

Convergence times for each attractor were calculated by simulating the model $M_{76777766}$ according to Eq. (6.1) starting from 10,000 initial conditions $\mathbf{x}(0)$, randomly selected from a uniform distribution on the $(S-1)$ simplex. Convergence times were calculated as the mean number of iterations required to satisfy the following criteria

$$
\sum_{i=1}^{8}\left|x_{i}(t-2)-x_{i}(t)\right|<0.001
$$


where $x_{i}(t)$ is the frequency of state $i$ at time $t$ (we used $x_{i}(t-2)$ instead of $x_{i}(t-1)$ because one of the attractors is a 2-cycle). The mean convergence time for the 2-cycle attractor is $22.4 \pm 5.8$ years (1 S.D.), for the CRI-HYM equilibrium is $31.1 \pm 6.7$ years, and for the SEA equilibrium is $397.6 \pm 308.2$ years.

\subsection{Dependence of species interactions on neigh- borhood size}

The above results are based on the assumption that species interactions operate over a spatial scale of $600 \mathrm{~cm}^{2}$ (i.e. the size of a single quadrat). Since the subtidal species in our model are sessile, however, they probably interact primarily with their neighbors. Thus species replacements are more likely to be effected by local neighborhood densities surrounding a patch than by the average population densities among quadrats (Pacala and Silander 1985, 1990).

Here we examine the effect of spatial scale on species interactions by subdividing the quadrats into either 4 or 8 subsections. In effect, making the spatial scale of the quadrats smaller is equivalent to decreasing the neighborhood size (NS) around each patch. Subdividing the quadrats into 4 sections created $4 \times 9=36$ sub-quadrats, each with an area of $150 \mathrm{~cm}^{2}$. Subdividing the quadrats into 8 sections created 72 sub-quadrats, each with an area of $75 \mathrm{~cm}^{2}$. Once the data was portioned in this manner, we followed the two step procedure outlined in section (6.4.3).

Table 6.6 shows the best fitting models using a neighborhood size of $150 \mathrm{~cm}^{2}$ and $75 \mathrm{~cm}^{2}$. In all cases, the second best fitting model for each column of $\mathbf{A}_{\mathbf{x}}$ has a $\triangle A I C$ value greater than 4 , suggesting that it has considerably less support than the best fitting model (Burnham and Anderson 1998). In general, decreasing the neighborhood size tends to reduce the number of species interactions in the model. For example, in the first column of $\mathbf{A}_{\mathbf{x}}$ (transitions from HYM), the $a_{i j}$ are a function

of four interspecific interactions when $N S=150 \mathrm{~cm}^{2}$, but only three interspecific 


\begin{tabular}{l|cc}
\hline \hline & \multicolumn{2}{|c}{ Neighborhood Size } \\
Column of A & $150 \mathrm{~cm}^{2}$ & $75 \mathrm{~cm}^{2}$ \\
\hline HYM & $M_{4}$ & $M_{3}$ \\
MYX & $M_{4}$ & $M_{2}$ \\
MYC & $M_{4}$ & $M_{4}$ \\
SEA & $M_{5}$ & $M_{5}$ \\
CRI & $M_{5}$ & $M_{5}$ \\
FIL & $M_{4}$ & $M_{2}$ \\
FG & $M_{4}$ & $M_{4}$ \\
BR & $M_{6}$ & $M_{3}$ \\
\hline \hline
\end{tabular}

Table 6.6: Best fitting models for each column of $\mathbf{A}$ obtained by dividing the quadrats into either 4 or 8 sub-quadrats. The sub-quadrats are equivalent to a neighborhood size of $150 \mathrm{~cm}^{2}$ and $75 \mathrm{~cm}^{2}$, respectively. The symbol $M_{h}$ specifics the model with the lowest $A I C$ value for the transition probabilities in each column of $\mathbf{A}$, where the subscript $h$ gives the optimal number of interspecific species interactions.

interactions when $N S=75 \mathrm{~cm}^{2}$. The best fitting model for the $150 \mathrm{~cm}^{2}$ neighborhood is $M_{44455446}$ and for the $75 \mathrm{~cm}^{2}$ neighborhood is $M_{32455243}$ (Table 6.6).

\subsubsection{Equilibrium distributions}

The nonlinear Markov chain models $M_{44455446}$ and $M_{32455243}$ have multiple- stable equilibrium states of attraction. Figure 6.3 shows the co-existing attractors at neighborhood sizes of $150 \mathrm{~cm}^{2}$ and $75 \mathrm{~cm}^{2}$. In both cases the dominant attractor is the CRI-HYM equilibrium. Two other attractors coexist when $N S=150 \mathrm{~cm}^{2}$, an equilibrium distribution dominated by Filograna (FIL equilibrium) and an equilibrium distribution dominated by Mycale (MYC equilibrium). Both of these equilibria, however, have much smaller basins of attraction than the CRI-HYM equilibrium. When $N S=75 \mathrm{~cm}^{2}$, the MYC equilibrium disappears completely, and the basin of the CRI-HYM attractor increases in size.

To examine the effect of neighborhood size on the ability of the model to predict the species distribution in the subtidal community, we calculated the distance between the various CRI-HYM equilibria and the observed species distribution shown in figure 
a.
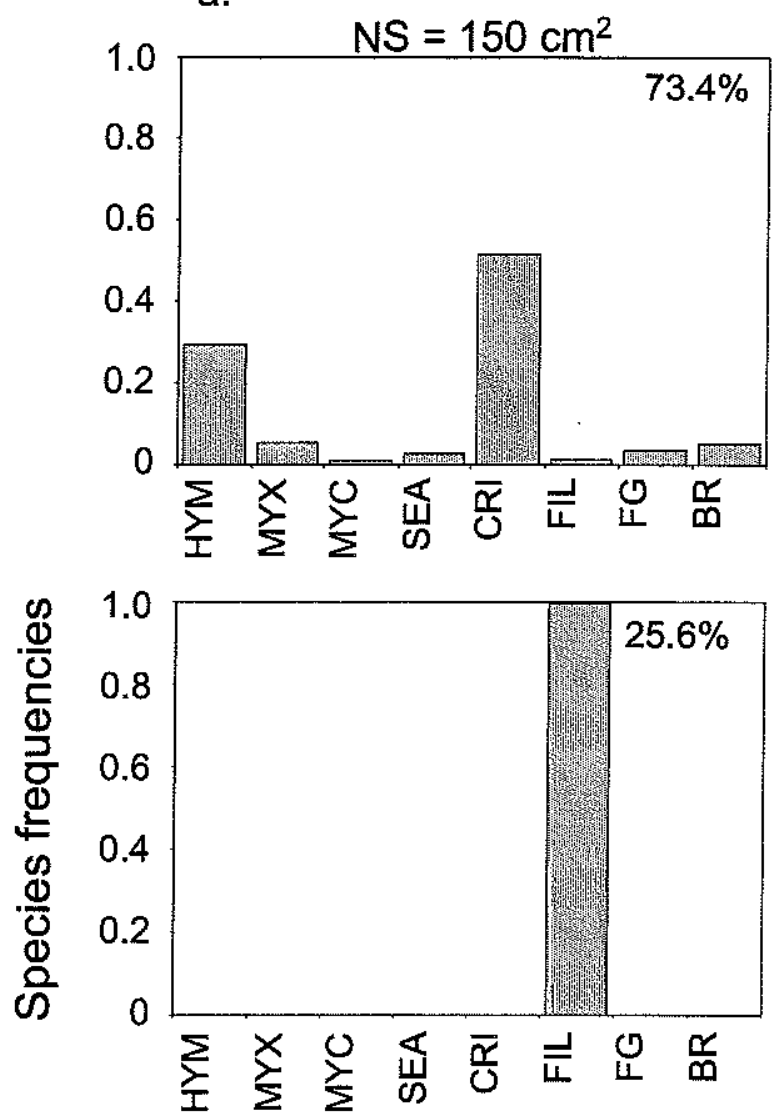

b.
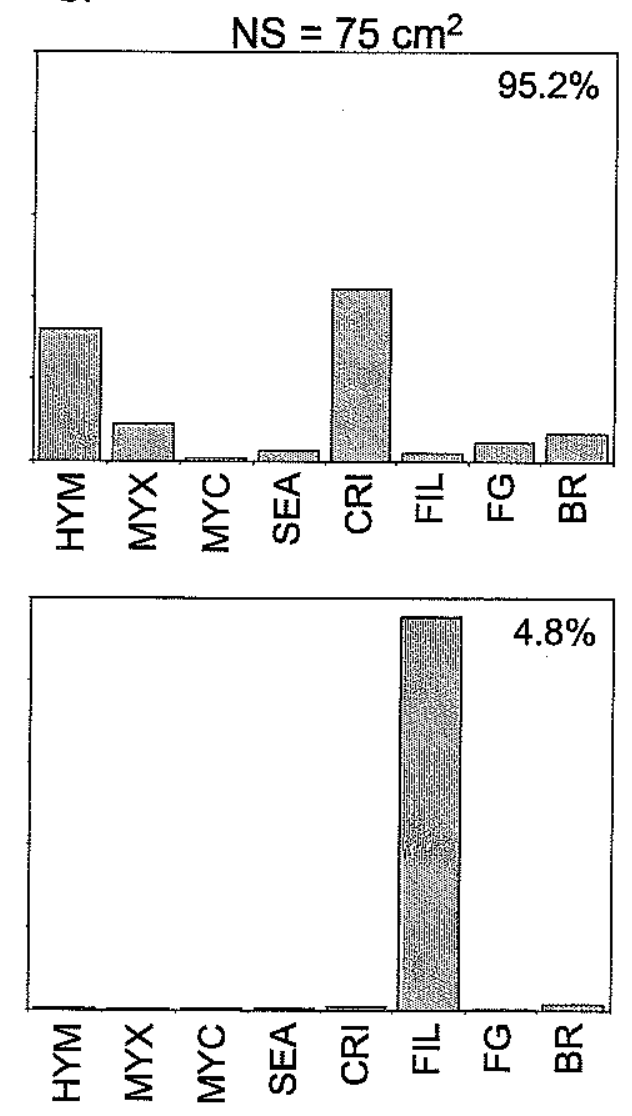

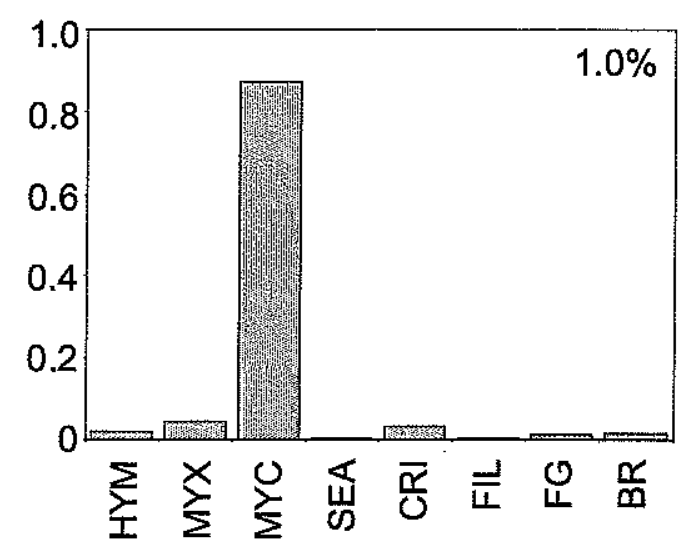

Figure 6.3: Coexisting attractors for the best fitting nonlinear Markov chain for neighborhood sizes of $a \mathrm{NS}=150 \mathrm{~cm}^{2}$ ), and $b . \mathrm{NS}=75 \mathrm{~cm}^{2}$ ). The percent in right hand corner of each histogram gives the proportion of times the model converged to each attractor starting from 10,000 random initial conditions. 


\begin{tabular}{lc}
\hline \hline Model & Distance $(D)$ \\
\hline Nonlinear & \\
$N S=600 \mathrm{~cm}^{2}$ & 0.68 \\
$N S=150 \mathrm{~cm}^{2}$ & 0.63 \\
$N S=75 \mathrm{~cm}^{2}$ & 0.44 \\
Linear & 0.19 \\
\hline \hline
\end{tabular}

Table 6.7: Distance between the predicted and observed equilibrium distribution for nonlinear and linear Markov chains. Comparisons for the nonlinear Markov chains use the CRI-HYM equilibrium distribution.

6.2. The distance $D$ is measured as

$$
D=\sum_{i=1}^{8}\left|\hat{x}_{i}-\hat{O}_{i}\right|
$$

where $\hat{x}_{i}$ is the predicted equilibrium frequency of species $i$ and $\hat{O}_{i}$ is the mean frequency of species $i$. Table 6.7 shows values of $D$ for neighborhood sizes of $600 \mathrm{~cm}^{2}$, $150 \mathrm{~cm}^{2}$, and $75 \mathrm{~cm}^{2}$, and for the linear Markov chain $M_{0}$ (i.e. the $a_{i j}$ are independent of species densities). While decreasing the neighborhood size in the nonlinear model decreases the distance between predicted and observed abundances, the linear model does a much better job at predicting community composition.

\subsubsection{Distribution of species interaction coefficients}

Figure 6.4 shows the distribution of species interaction coefficients for the best fitted models, estimated at different neighborhood sizes. In all cases the mean value is approximately 0 , but the variance decreases with decreasing neighborhood size from $105.6\left(\mathrm{NS}=600 \mathrm{~cm}^{2}\right.$.), to $44.2\left(\mathrm{NS}=150 \mathrm{~cm}^{2}\right)$, to $19.52\left(\mathrm{NS}=75 \mathrm{~cm}^{2}\right)$. Thus, as the neighborhood size decreases, the proportion of species interactions that are relatively weak increases. 

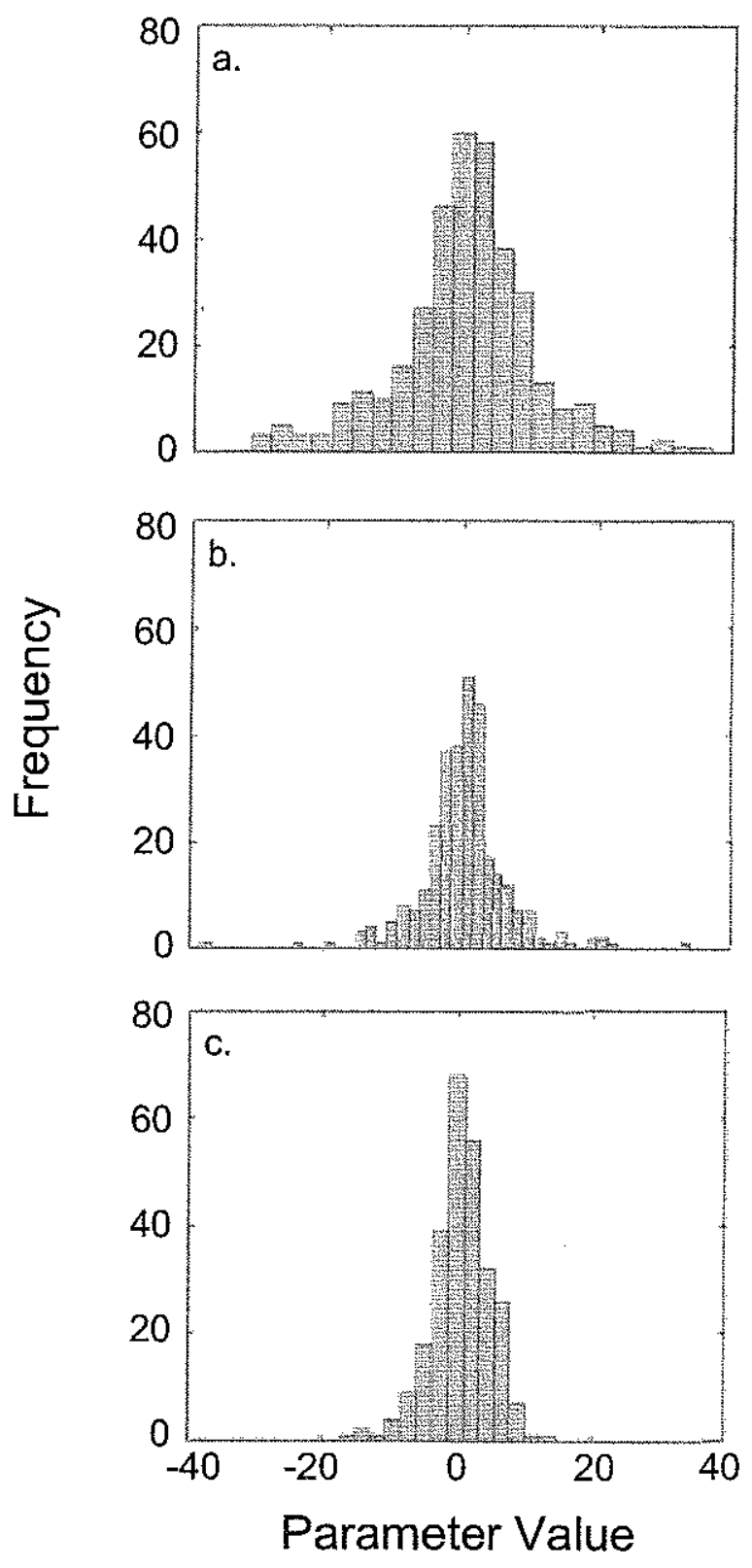

Figure 6.4: Distribution of interaction coefficients for the best fitting nonlinear Markov chain estimated different size neighborhoods. a. NS $=600 \mathrm{~cm}^{2}$ ). The total number of interaction coefficients is $371 . b . N S=150 \mathrm{~cm}^{2}$ ). The total number of interaction coefficients is 308 . $c$. NS $=75 \mathrm{~cm}^{2}$ ). The total number of interaction coefficients is 266 . 


\begin{tabular}{l|ccc}
\hline & \multicolumn{3}{|c}{ Neighborhood Size } \\
Equilibrium & $600 \mathrm{~cm}^{2}$ & $150 \mathrm{~cm}^{2}$ & $75 \mathrm{~cm}^{2}$ \\
\hline CRI-HYM & $31.15(6.73)$ & $76.41(26.78)$ & $30.56(26.28)$ \\
FIL & - & $9.61(2.35)$ & $13.19(3.03)$ \\
\hline \hline
\end{tabular}

Table 6.8: Mean convergence time to the CRI-HYM and FIL equilibriums, based on 10,000 simulations of the nonlinear Markov chain associated with each Neighborhood size. Values in parenthesis are 1 standard deviation (see text for details).

\subsubsection{Convergence times and patterns of succession}

The mean convergence times for the models $M_{44455446}\left(\mathrm{NS}=150 \mathrm{~cm}^{2}\right)$ and $M_{32455243}$ (NS $=75 \mathrm{~cm}^{2}$ ) to the CRI-HYM equilibrium and the FIL equilibrium are shown in Table 6.8. The mean convergence time to the CRI-HYM equilibrium is 3 to 8 times longer than the convergence time to the FIL equilibrium. There is no general relation between convergence times and neighborhood size. Convergence time for the CRIHYM equilibrium is longest when NS $=150 \mathrm{~cm}^{2}$ (intermediate spatial scale), while for the FIL equilibrium it is longest when $\mathrm{NS}=75 \mathrm{~cm}^{2}$.

Figure 6.5 shows the pattern of succession starting from an initial condition in which the abundance of bare rock is set to one, and abundances of all the species are set to zero. This represents succession following the total destruction of the existing community. When the neighborhood size is $600 \mathrm{~cm}^{2}$ the community converges almost immediately to the periodic 2-cycle attractor (Fig. 6.5a.). When the neighborhood size is $150 \mathrm{~cm}^{2}$ or $75 \mathrm{~cm}^{2}$, however, the community converges to the CRI-HYM equilibrium (Fig. $6.5 \mathrm{~b}, \mathrm{c}$ ). Note that the time track of colonization differs between many of the species. For example, Filograna, the sea anemones, and the functional group (FG) reach an early peak in abundance within $2-4$ years and then drop off fairly sharply before they approach their equilibrium. The sponges Hymedesmia and Myxilla, on the other hand, tend to increase monotonically towards their equilibrium abundance. Finally, Crisia increases monotonically when $N S=150 \mathrm{~cm}^{2}$, but shows an early peak in abundance followed by a slow decline towards equilibrium when $N S=75 \mathrm{~cm}^{2}$. 
a.
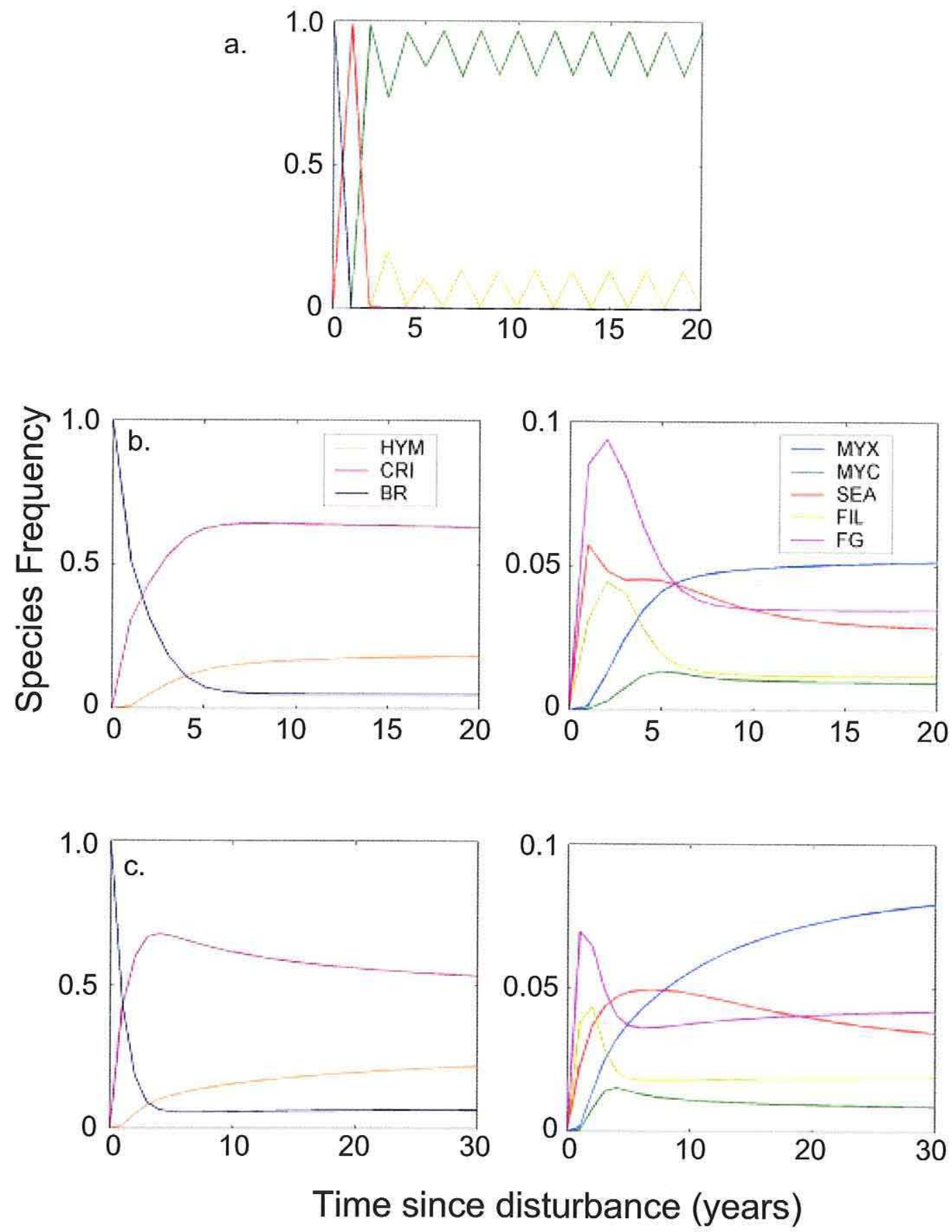

Figure 6.5: Simulation of state abundances over time, following complete destruction of the community (initial condition equals $100 \%$ bare rock). $a$. NS $=600 \mathrm{~cm}^{2}$ ). $b$. $\left.\left.\mathrm{NS}=150 \mathrm{~cm}^{2}\right) . c . \mathrm{NS}=75 \mathrm{~cm}^{2}\right)$. 


\subsubsection{Rates of local succession}

A Markov chain describes two spatial scales-the dynamics of the community and the local dynamics of patches. At equilibrium the proportion of patches in each state is constant, however, individual patches change states continuously through time.

Once the community converges to an equilibrium distribution the matrix $\mathbf{A}_{\mathbf{x}}$ remains constant. From the equilibrium matrix $\hat{\mathbf{A}_{\mathbf{x}}}$ we investigate rates of successional change at the spatial scale of a patch by calculating mean turnover rates and Smouchowski recurrence times for each state.

- The mean turnover rate describes the probability that a randomly selected patch changes state between $t$ and $t+1$, and is given by

$$
\sum_{i=1}^{s} w_{i}\left(1-a_{i i}\right)
$$

where $w_{i}$ is the $i$ th element of the dominant eigenvector, and $\left(1-a_{i i}\right)$ is the probability that a patch in state $i$ changes states in the time interval from $t$ to $t+1$ (Iosifescu 1980).

- The Smouchowski recurrence time $\theta_{i}$ of state $i$ is the time elapsing between a patch leaving state $i$ and then returning to it again. Its mean is given by

$$
\bar{\theta}_{i}=\frac{1-w_{i}}{w_{i}\left(1-a_{i i}\right)}
$$

(Iosifescu 1980).

Table 6.9 shows mean turnover rates at the various equilibrium points. Patch turnover rates are much greater when the system has converged to the CRI-HYM equilibrium, than when it has converged the FIL or MYC equilibrium. In the case of the CRI-HYM and FIL equilibriums, the predicted turnover rate increases as the spatial scale of the density dependence decreases. 


\begin{tabular}{l|ccc}
\hline & \multicolumn{3}{|c}{ Density Spatial Scale } \\
Equilibrium & $300 \mathrm{~cm}^{2}$ & $150 \mathrm{~cm}^{2}$ & $75 \mathrm{~cm}^{2}$ \\
\hline CRI-HYM & 0.2700 & 0.3112 & 0.3655 \\
FIL & - & 0.0031 & 0.0786 \\
MYC & - & 0.1673 & - \\
\hline \hline
\end{tabular}

Table 6.9: Mean turnover rate of patches when the subtidal community is at an equilibrium point. Turnover rates give the probability that a randomly chosen patch changes states in one time interval. The turnover rates in each column correspond to the best fit nonlinear Markov chain for a given spatial scale of density dependence (see text for details).

Figure 6.6 shows Smouchowski recurrence times for each species at the CRI-HYM equilibrium and the FIL equilibrium. In general, $\bar{\theta}_{i}$ decreases as the neighborhood size decreases. This is especially true in the case of the FIL equilibrium, where the recurrence time for many species decreases by as much as ten-fold when the $N S$ decreases form $150 \mathrm{~cm}^{2}$ to $75 \mathrm{~cm}^{2}$.

\subsection{Bifurcation Analysis}

When the neighborhood size of a patch is small, the nonlinear Markov chain always converges to a stable equilibrium. One of the signatures of nonlinear models, however, is their ability to produce periodic cycles, aperiodic oscillations and chaos. Shifts in parameter values can cause bifurcations from one of these behaviors to another (e.g., May 1974,1976, Hastings and Powell 1991; Pascual 1993, Costantino et al. 1995; Caswell and Neubert 1998). Here we look for bifurcations caused by changes in the strength of the interactions among species. We focus on the nonlinear Markov chain $M_{32455243}$, parameterized using a neighborhood size of $75 \mathrm{~cm}^{2}$.

The interaction coefficients for a given species can be changed in the following way. Let $\mathbf{C}^{(k)}$ be a coefficient matrix whose $(\mathrm{i}, \mathrm{j})$ element is the interaction coefficient associated with species $k$ in the function $a_{i j}(\mathbf{x})$. The coefficient matrix for Hymedesmia 


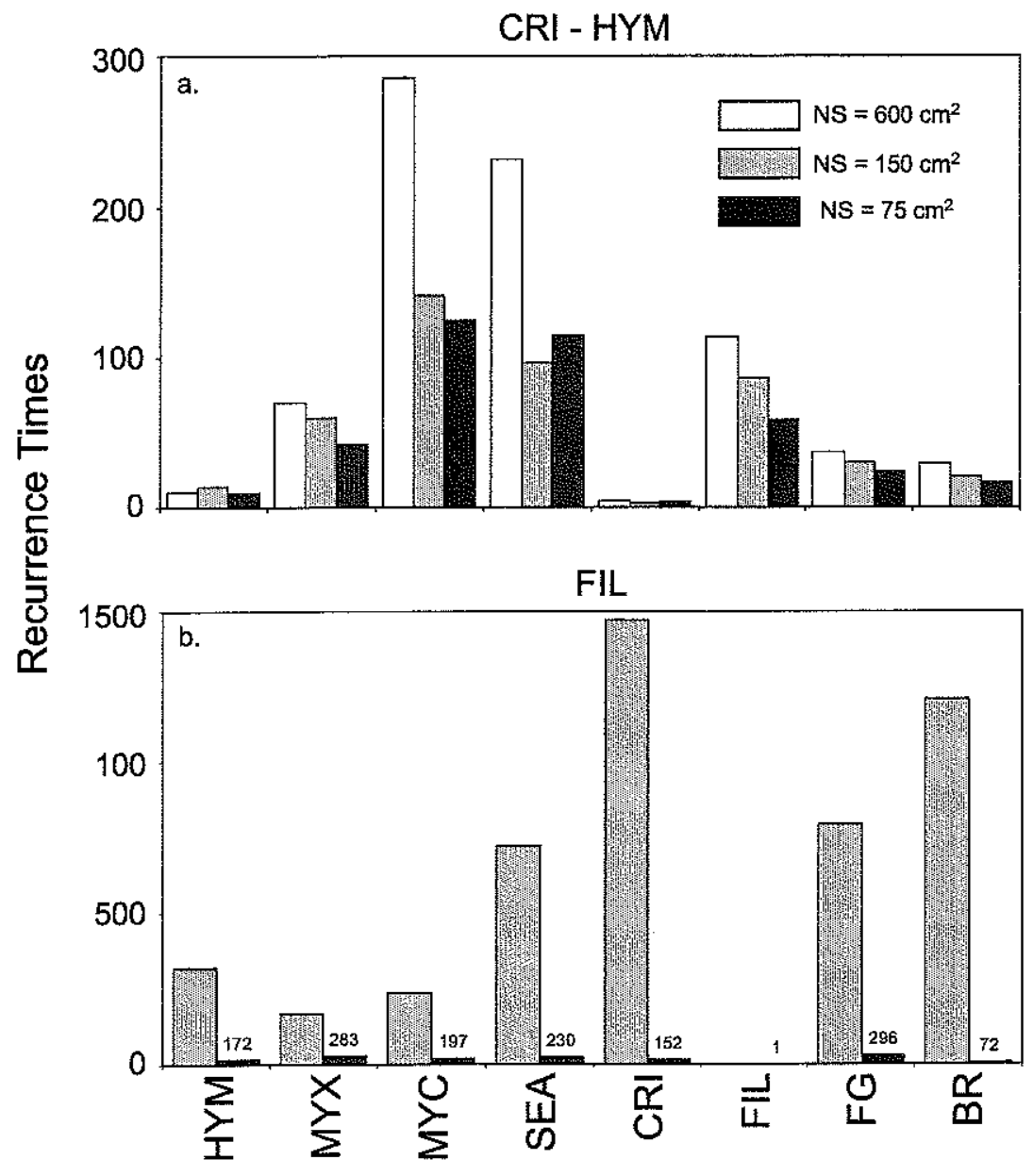

Figure 6.6: Smouchowski recurrence times for each patch state $\bar{\theta}_{i}$ at equilibrium. The different colored bars in the histograms represent recurrence times for different size neighborhoods. $a$. Smouchowski recurrence times for state $i$ after the community has converged to the CRI-HYM equilibrium. $b$. Smouchowski recurrence times for state $i$ after the community has converged to the FIL equilibrium. 
$(k=1)$ is shown below

$$
\mathbf{C}^{(1)}=\left(\begin{array}{rrrrrrrr}
0.77 & 2.52 & -2.41 & 0.46 & 2.85 & 2.41 & 1.32 & 2.06 \\
-0.46 & 1.27 & - & -0.44 & -0.44 & - & - & - \\
-1.85 & - & - & - & 0.21 & -2.87 & 0.97 & 1.93 \\
- & - & -5.14 & - & -0.08 & - & -0.34 & -4.51 \\
-0.44 & 1.64 & 0.20 & - & 0.90 & - & -1.06 & - \\
- & - & - & - & - & - & - & -0.67 \\
-0.11 & - & - & - & -0.83 & - & 3.44 & -
\end{array}\right)
$$

where the element $c_{11}^{(1)}$ is the value of the interaction coefficient associated with $x_{1}$ (i.e. the density of Hymedesmia) in the function $a_{11}(\mathbf{x}), c_{21}^{(1)}$ is the value of the interaction coefficient associated with $x_{1}$ in the function $a_{21}(\mathrm{x})$, etc. The dashed symbols indicate transition probabilities that are independent of the density of Hymedesmia. An example is the function $a_{32}(\mathbf{x})$ which represents the probability that Myxilla is replaced by Mycalle.

To obtain a bifurcation diagram for species $k$ we proportionally increase the interaction coefficients by a factor $p$

$$
\mathbf{C}_{p}^{(k)}=\mathbf{C}^{(k)}+\left(\mathbf{C}^{(k)} \times p\right)
$$

and then insert the new coefficient values given in $\mathbf{C}_{p}^{(k)}$ into the functions $a_{i j}(\mathbf{x})$ to obtain a new transition matrix $\mathbf{A}_{\mathbf{x}}{ }^{(k, p)}$ (the superscript $(k, p)$ indicates that the interaction coefficients associated with species $k$ in the functions $a_{i j}$ have been increased by a factor of $p$ ). Bifurcation diagrams were obtained by starting the system at an equilibrium point and then increasing the value of $p$ from 0 to 10 in increments of 0.01. At each increment, we iterated the model 500 times to remove any transient dynamics, and then plot 50 iterates of the value of species $k$ (i.e. $x_{k}$ ).

Figure 6.7 shows a set of bifurcation diagrams starting from the CRI-HYM equi- 
librium, in which the value of $x_{k}$ is plotted as a function of the proportional change $(p)$ in the interaction coefficients of species $k$. Increases in the interaction strength of Crisia and Mycale produce the most dramatic bifurcation patterns, including periodic cycles, phase locking and chaotic dynamics. Increases in the interaction strength of Myxilla results in a bifurcation from a stable equilibrium to a 2-cycle at $p=3.67$, while increases in the interaction strength of Filograna and the sea anemones produce a sharp transition in their frequencies (from less than 0.05 to 1 ) at $p$ equals 3.43 and 0.78 , respectively. For the remaining species, increasing $p$ results in a smooth change in equilibrium abundance, but no bifurcations.

The most dramatic bifurcation patterns are associated with Crisia (Figure 6.8). If we start the system in the basin of attraction of the CRI-HYM equilibrium (Fig. 6.8a), the attractor is a fixed point for $p<2.67$. At $p=2.67$ a Hopf bifurcation occurs in which the fixed point is replaced by an invariant circle. At $p \approx 3.51$, the invariant circle is replaced by a 3 -cycle. Increasing $p$ further results in period doublings and eventually leads to chaos. In the chaotic region of parameter space, species densities are aperiodic in time and show sensitive dependence to initial conditions (Fig. 6.9). When we start the system in the basin of attraction of the FIL equilibrium, the attractor remains a stable fixed point until $p \approx 7.09$ (Fig. 6.8b). At $p>7.09$ the fixed point becomes unstable and the system falls into the basin of attraction of the chaotic attractor.

Increasing the interaction strength of the remaining species has no effect when we start the system in the basin of attraction of the FIL equilibrium. Thus even though the basin of attraction of the FIL equilibrium is comparatively small (relative to the CRI-HYM equilibrium), the FIL equilibrium is more stable to large perturbations in interaction strengths.

Because many interaction coefficients are positive, increasing the interaction strength of one species increases the relative abundance of other species in the community: Figure 6.10 shows how the abundance of Hymedesmia, Mycale, and the FG species vary 

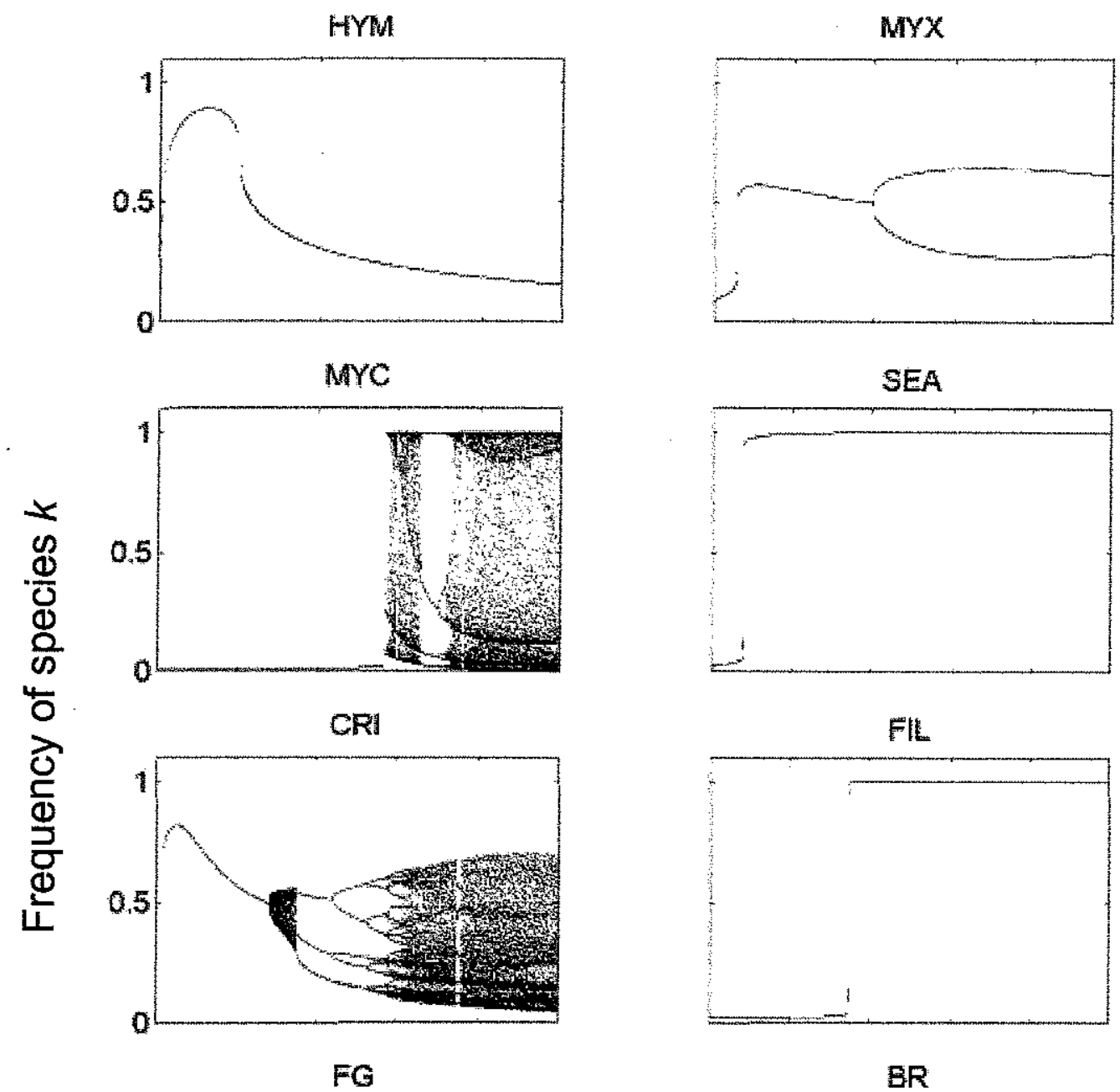

트
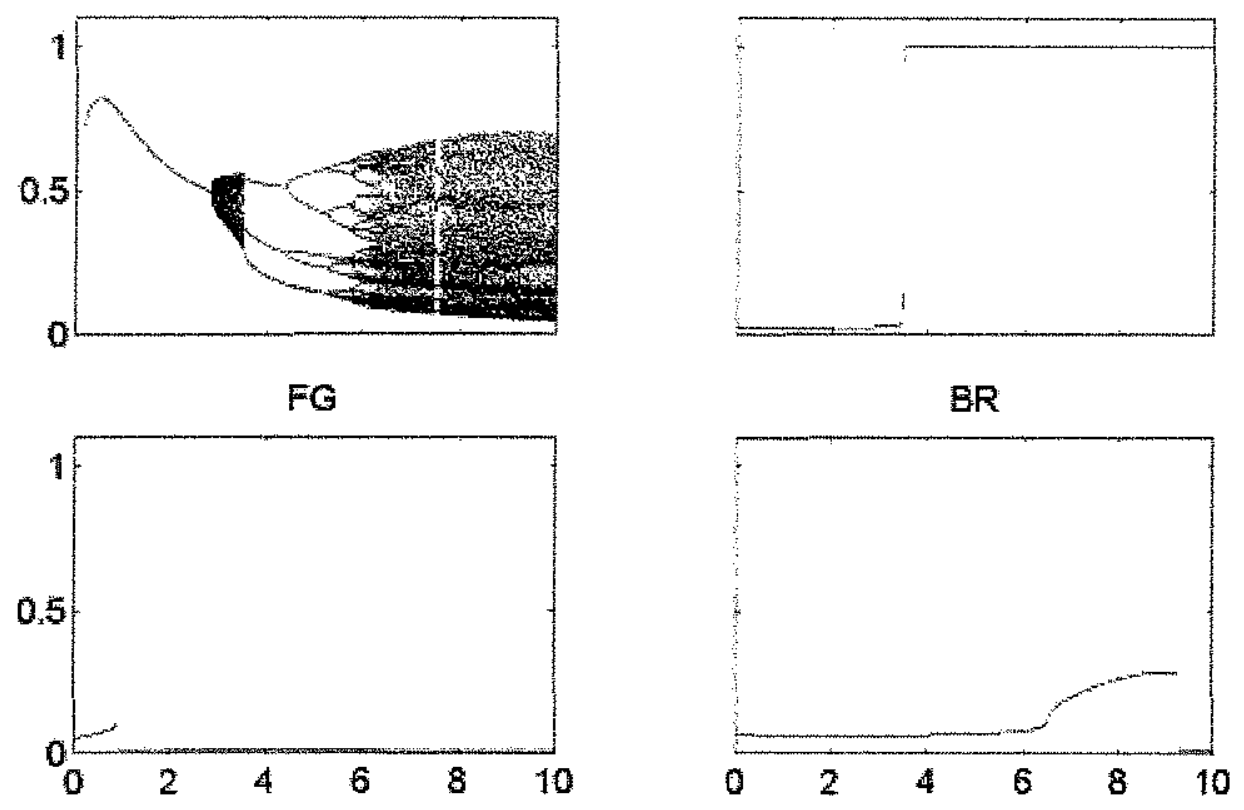

\section{Proportional increase $(p)$ in the interaction coefficients of state $k$}

Figure 6.7: Bifurcation diagrams plotting the frequency of each species $k$ ( $k=$ $1, \ldots, 8)$ as a function of the proportional increase $(p)$ in the interaction coefficients of species $k\left(\mathrm{NS}=75 \mathrm{~cm}^{2}\right)$. Each bifurcation diagram was generated by starting from an initial condition in the basin of attraction of the CRI-HYM equilibrium. 


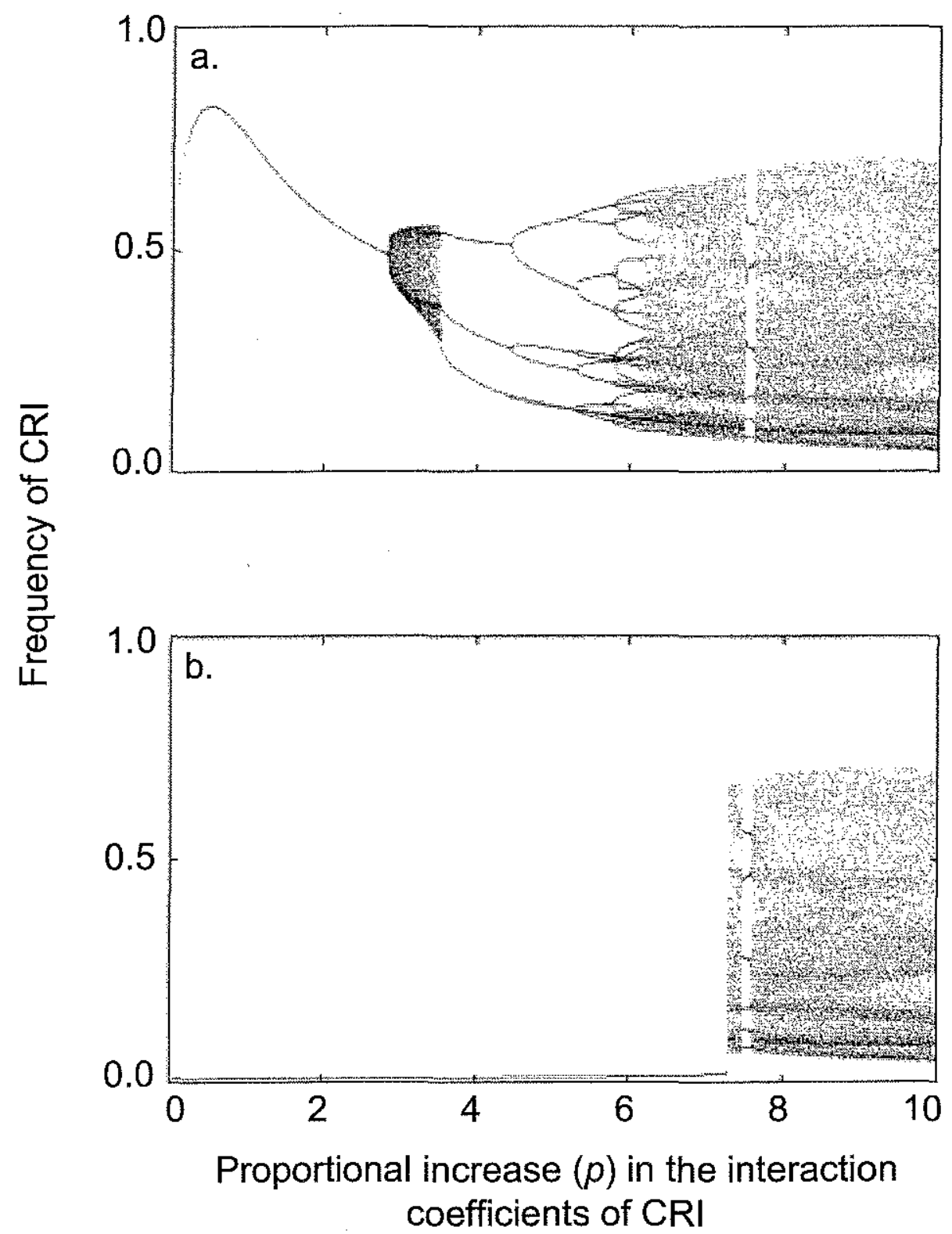

Figure 6.8: Bifurcation diagrams plotting the frequency of Crisia on the attractor as a function of the proportional increase $(p)$ in the interaction coefficients of Crisia $\left(\mathrm{NS}=75 \mathrm{~cm}^{2}\right)$. a. Bifurcation diagram starting on the CRI-HYM attractor. $b$. Bifurcation diagram starting on the FIL attractor. 


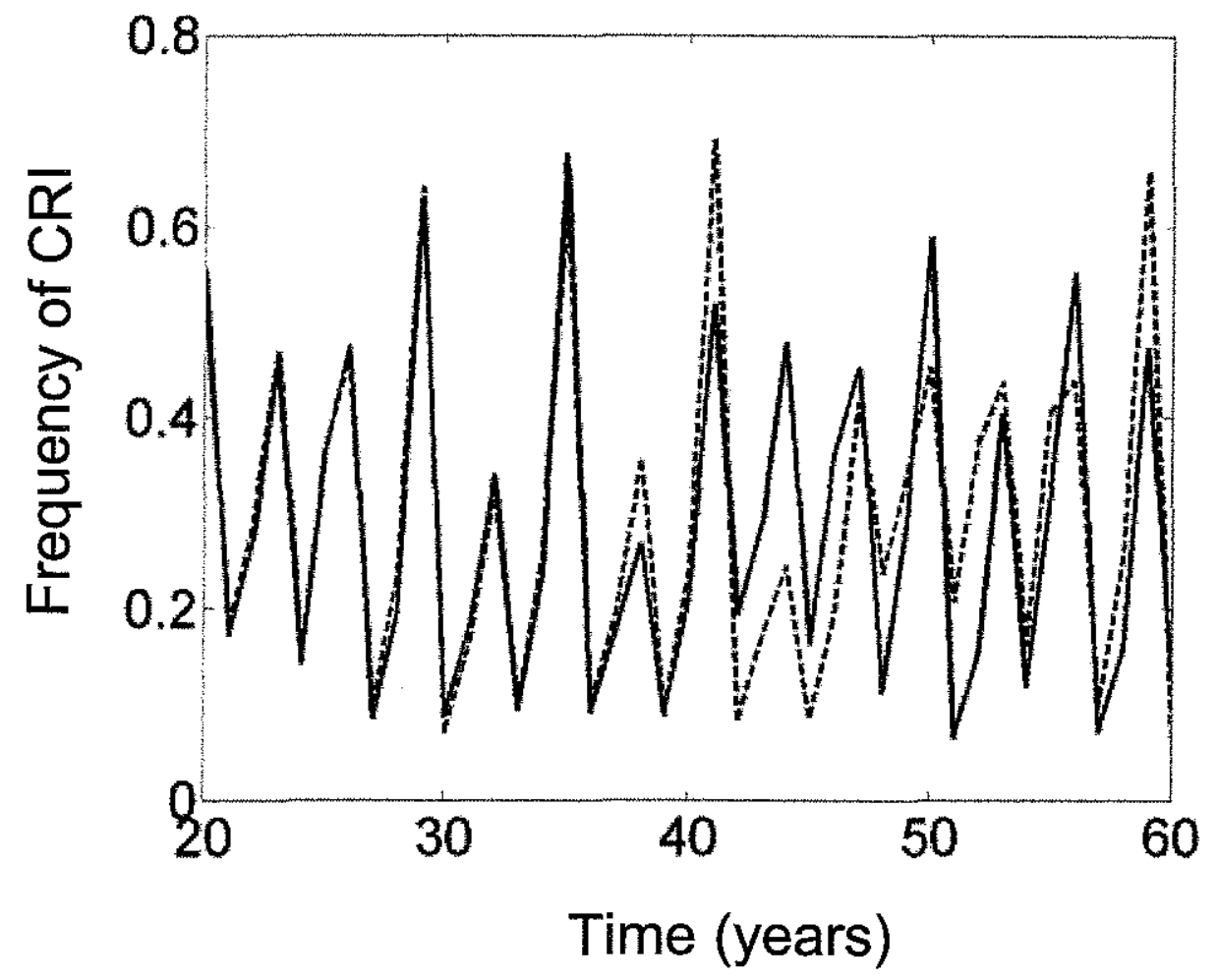

Figure 6.9: Sensitivity to initial conditions. Time series plot of Crisia showing that temporal solutions diverge for small initial differences. Two trajectories of Crisia are shown in which the initial frequency of Crisia differs by only $0.001(p=8)$. 
as a function of the proportional increase in the interaction strength of Hymedesmia. Increasing $p$ from 0 to 1 increases the abundance of Hymedesmia. At $p \approx 1$, however, there is an abrupt decline in Hymedesmia accompanied by a sharp increase in the FG species. When $p>1$, Hymedesmia abundance is a decreasing function of $p$ while Mycale abundance is a monotonically increasing function of $p$. Thus, increasing the interaction strength of Hymedesmia enables Mycale and the FG species to increase their abundance at the expense of Hymedesmia.

\subsection{Discussion}

There have been very few empirical studies that have tried to quantify interaction strengths among assemblages of species. Most studies have relied on estimating per capita interaction strengths through single species manipulation experiments (e.g., Fowler 1981, Menge and Farrell 1989). The problem with this approach is that there may be a large number of interactions and that the prevalence of indirect effects in most communities (the effects of one species on another via its effect on a third species) means that pair-wise estimates of interaction strengths are not additive. Thus, extrapolating results from single species manipulation experiments to whole communities are likely to be unsuccessful (Wilbur 1972; Neil 1974; Wilbur and Fauth 1990; Wooten 1993).

The methods presented here allowed us to estimate density-dependent interactions in an unmanipulated community using spatial time series data. By focusing on patch transition probabilities, instead of individual species, we were able to simultaneously estimate interaction coefficients for all species states in our model using maximum likelihood methods. Any indirect effects are automatically incorporated into the model because interaction coefficients are estimated by taking into account the dynamics of the community as a whole. This is not true for species removal experiments. 


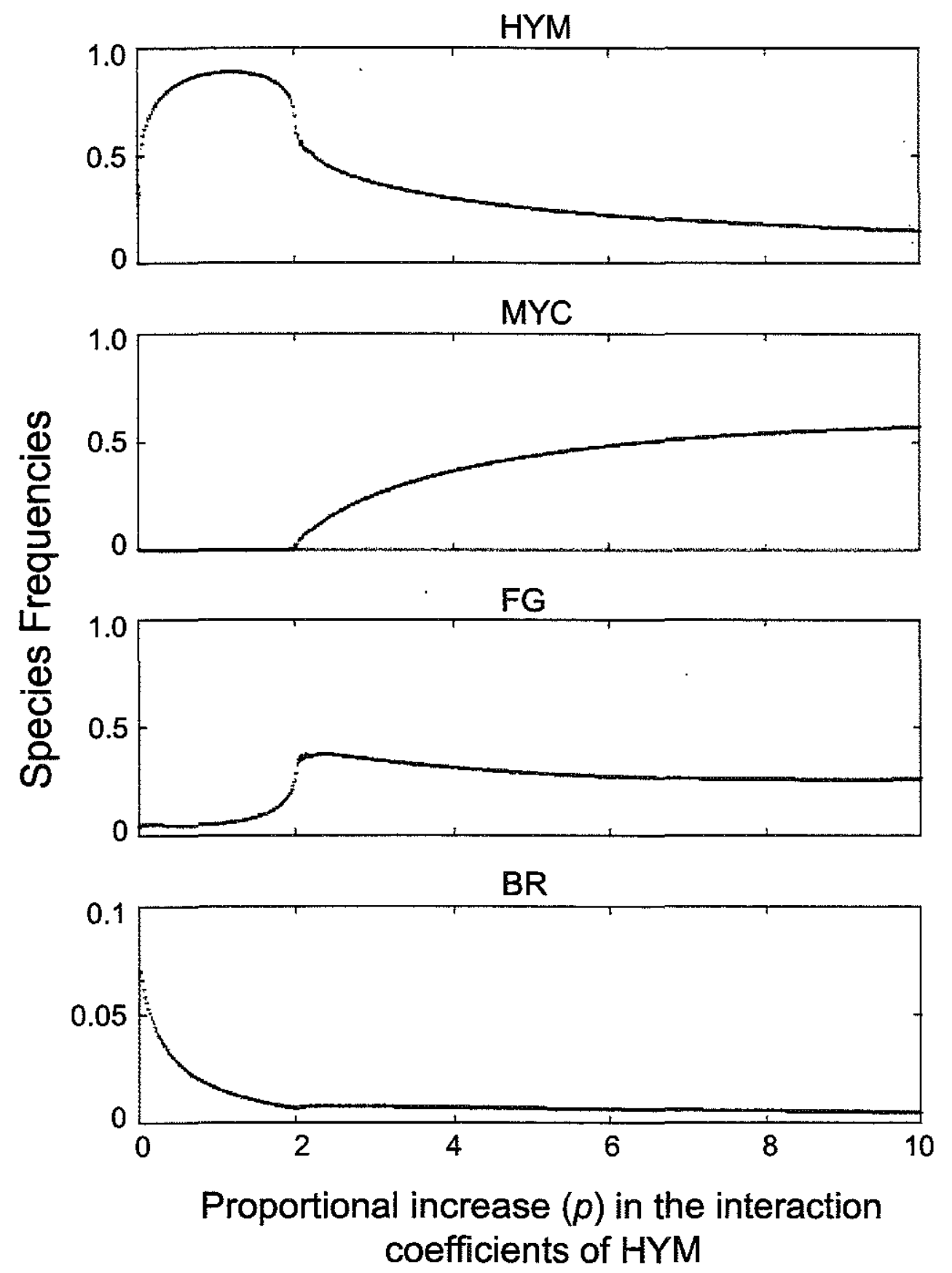

Figure 6.10: Bifurcation diagrams for the abundance of the states HYM, MYC, FG and $\mathrm{BR}$ as a function of the proportional increase $(p)$ in the interaction coefficients of Hymedesmia. 
Another difference between our methods and species removal experiments is that removal experiments tend to focus on interesting or significant interactions, which means they are usually selected because they are strong (Lawton 1992). Our analysis shows not only that the best fitting models contains a large number of species interactions but also that most of these interactions are relatively weak. This result is consistent with several empirical studies of plant communities showing they are dominated by a large number of competitively weak interactions (e.g., Schoener 1983, Mahdi et al. 1989, Polis 1991).

Parameter estimation: The two-step method outlined in section 6.4 .3 allowed us to identify the best fitting nonlinear Markov models, within an extremely large parameter space, in a relatively short period of time (2 to 4 days depending on the size of the neighborhood). The reason for this is two fold. First, the binary logistic regression method greatly reduces the number of species combinations one needs to test to find the best fit for a given model. For example, to find the best $M_{1}$ model for column $j$, one needs to identify the species whose density has the greatest effect on the replacement of species $j$ by species $i, i=1, \ldots, S$ (i.e. the highest ranked species for each $a_{i j}(\mathbf{x})$ ). Since the number of possible choices per transition probability is $S-1$, the number of possible species combinations we need to test to find the highest ranked species for all the transition probabilities in column $j$ is $S(S-1)$. In contrast, if we used polychotomous logistic regression to perform the same task, the number of possible combinations we would need to test is $(S-1)^{(S-1)}$. The problem, of course, only gets worse as the number of species interactions increases.

The second reason the two-step method shortens the search time is that maximization of Eq. 6.11 (binary likelihood function) is much faster than Eq. 6.6 (polychotomous likelihood function). Thus, the binary regression methods enable us to quickly identify the relative ranking of the species, according to their effect on the replacement of species $j$ by species $i$, for each entry in $\mathbf{A}_{\mathbf{x}}$. Once the species rankings 
are determined, however, the interaction coefficients associated with a given model $M_{h}$ are found fairly quickly using the polychotomous regression method (since we only need to maximize Eq. 6.6 once for a given set of species interactions).

Community dynamics: Simulation of the nonlinear Markov chain suggested that the subtidal community is capable of periodic orbits and multiple equilibria. This suggests that large perturbations in species abundance (caused by random climate change or biological disturbances) may result in ecological shifts from one equilibrium region to another. This pattern of multiple equilibrium states has been observed in several empirical studies of natural communities (Holling 1973, 1992; Sutherland 1974; Levin 1976; May 1977; Hughes 1994). Sutherland (1974) and Connell and Slatyer (1977) have extensively reviewed work on the marine rocky intertidal, on coral reefs, on freshwater lakes, and on terrestrial plant communities, and concluded that community structure is indelibly tied to historical events and therefore multiple equilibrium states are an undeniable reality of natural systems.

Our model of the subtidal community corroborates these conclusions and suggests that large-scale phase shifts in community composition are possible. If we assume that the current state of subtidal communities in the Gulf of Maine falls within the basin of attraction of the CRI-HYM equilibrium, then any change in the physical environment, which pushes the system out of that basin, will drastically change the structure of the community. All of the other equilibrium states in our model predict complete domination of the substrate by a single species (regardless of the density spatial scale used to parameterize the model). Thus perturbations that shift the community towards a new equilibrium will drastically reduce the diversity of the community. Huges (1994) has documented such an event in coral reef communities off Jamaica, where overfishing, disease, and hurricane damage have combined to destroy most corals, resulting in dramatic phase shift to a low diversity system dominated by fleshy macroalgae. 
Distribution of interaction coefficients: We found that interaction strengths in the subtidal community were symmetrically distributed around zero, with interaction coefficients equally as likely to be positive as negative. While negative coefficients probably represent competition between species for space, positive coefficients represent not only mutualistic interactions but also indirect species effects (e.g., Callaway 1997, Hodge et al.. 1999, Levine 1999). If the presence of species $X$, inhibits species $Y$ which has a negative effect on species $Z$, then the interaction coefficient corresponding to species $X$ in the function $a_{Z j}(\mathbf{x})$ is likely be positive. Paine (1992) found similar indirect positive interactions between intertidal invertebrates (chitons and limpets) and two species of brown algae (Hedophyllum sessile and Alaria ssp.). In this case the invertebrates prey on a crustose coralline algae, which in turn inhibits the recruitment of $H$. sessile and Alaria. Thus, the actions of the invertebrate predators have a positive effect on the brown algae because it increases the recruitment rate of $H$. sessile and Alaria onto the substrate surface.

The effects of neighborhood size: The number of species interactions and the distribution of interaction strengths in our model is dependent on neighborhood size we chose to parameterize the model. When density effects are measured over smaller spatial scales the number of interactions decreases and interaction strengths became weaker (Fig. 6.4). The pattern of species interactions specified by our model is similar to several recent empirical community studies which show that most species have weak effects on the abundance of other species, while only a few have strong effects (Paine 1992, Fagan and Hurd 1994, Raffaelli and Hall 1996; Wootton 1997).

The neighborhood size used to estimate species densities also affected the behavior of the nonlinear Markov chain. When we parameterize the model using an neighborhood size of $600 \mathrm{~cm}^{2}$, the dominant behavior of the system is a two-cycle, in which the community is dominated by the sponge Mycale. As we decreased the neighborhood size, however, the best-fitting model predicts a more stable and diverse community 
structure (CRI-HYM equilibrium). This may be due to the increase in the number of weak interactions between species. Weak species links have been shown to dampen oscillations in theoretical food web models, thus reducing the probability that species go extinct (McCann et al. 1998).

Finally, neighborhood size also plays an important role in measurements of local patch dynamics. Mean turnover rates and species recurrence times both increase as the neighborhood size decreases. This result points out one of the fundamental dilemmas in ecology; identifying the characteristic scale at which local interactions operate to produce observed community patterns at larger temporal and spatial scales (Levin 1992; Pascual and Levin 1999).

Nonlinear Dynamics: The bifurcation analysis allowed us to examine what would happen to the community if the intensity of interactions associated with one species were increased. Increases in the strength of the interaction of the bryozoan Crisia (and to a lesser extent Mycale) with the rest of the community lead to periodic oscillations and chaotic dynamics. While chaos has been found in numerous theoretical community models (e.g., Gilpin 1979, Hastings and Powell 1991, Kot et al 1992, Caswell and Neubert 1998), this is the first time it has been reported in a Markov chain model for community competition.

The bifurcation analysis also shows that increasing the interaction strength of a species does not guarantee its abundance will increase. For example, increases in the strength of the interaction of Hymedesmia with the rest of the community actually lead to a decrease in its abundance (Fig. 6.10). This occurred because Hymedesmia has a strong negative effect on the abundances of Crisia, Myxilla, Filograna, and the sea anemones, which in turn have a negative effect on the abundance of Mycale. If the interactions of Hymedesmia with other species become too strong, then the species inhibiting Mycale are almost completely wiped out. This allows Mycale to increase its abundance in the community and actually inhibit Hymedesmia. 


\subsection{Conclusion}

Most applications of Markov chain models to marine systems have been limited to linear models, in which transition probabilities are time-invariant. Patch transitions, however, certainly depend on species abundances. To assess the importance of such factors in a rocky subtidal community, we constructed a nonlinear Markov chain by making patch transition probabilities a function of species densities. We introduced a two-step method, based on logistic regression analyses for binary and polychotomous variables, that allowed us to estimate the density dependence of the elements $\mathbf{A}_{\mathbf{x}}$. Parameters for these functions were estimated using maximum likelihood analysis and the best models chosen using $A I C$ methods.

By applying this methodology to the subtidal data set, we showed that the community possess multiple equilibrium states, that the distribution of species interactions are symmetrically distributed around zero (and mostly weak), and that the behavior of the model depends on the spatial scale (neighborhood size) at which the parameters are estimated. These results illustrate the complexities involved in predicting the behavior of any species assemblage. First, systems with multiple equilibria can change radically given a large perturbation (May 1977) and thus future states of the community are highly dependent on random environmental events. Second, choosing the correct spatial scale to parameterize the model is not a simple task, and nonlinear models parameterized using different density spatial scales can give entirely different results (Pascual and Levine 1999).

Our approach differs from traditional methods of measuring interaction strengths because we are concerned with their effect on patch transitions as opposed to species abundances. The advantage of our methodology is that it allows us to simultaneously estimate a set of interaction coefficients based solely on time series data. Because of the nonlinearities inherent in ecological systems, the effect of changes in interaction strengths and/or species densities can only be understood if all possible combinations of interactions are estimated at the same time (Bender et al. 1984). Pair-wise 
estimations of interaction strengths (via species removal experiments) provide little information about how species will interact when they co-occur within an assemblage of organisms, and what effect those interactions will have on the dynamics of the community (Wootton 1993). 


\section{References}

Acevedo, M. 1982. On Horn's Markovian model of forest dynamics with particular reference to tropical forests. Theoretical Population Biology, 19:230-250.

Akaike, H. 1973. in Intermational Symposium on Information Theory, eds. B.N. Petran \& F. Csaki. Akademiai Kiado, Budapest, Hungary

Ayling, A.M. 1981. The role of biological disturbance in temporal subtidal encrusting communities. Ecology, 62:830-847.

Barradas, I. and J.E. Cohen. 1994. Disturbances allow coexistence of competing species. Journal of Mathematical Biology, 32:663-676.

Barradas, I., H. Caswell and J. E. Cohen. 1996. Competition during colonization vs. competition after colonization in disturbed environments: A metapopulation approach. Bulletin of Mathematical Biology, 58:1187-1207.

Bertness, M.D. 1989. Intraspecific competition and facilitation in a northern acorn barnacle population. Ecology, 70:257-268.

Callaway, RM. 1997. Positive interactions in plant communities and the individualisticcontinuum concept. Oecologia, 112:143-149

Caswell H., and J.E. Cohen. 1991a. Communities in patchy environments: a model of disturbance, competition, and heterogeneity. In J. Kolasa and S.T.A. Pickett (eds.) Ecological Heterogeneity. Springer-Verlag, New York, pp. 97-122.

Caswell H., and J.E. Cohen. 1991b. Disturbance, interspecific interaction and diversity in metapopulations. Biol. J. Linnean Soc., 42:193-218.

Caswell, H. and J.E. Cohen. 1993. Local and regional regulation of species-area relations: a patch-occupancy model. pp. 99-107 in R.E. Ricklefs and D. Schluter (eds.) Species diversity in ecological communities. University of Chicago Press, Chicago. 
Caswell, H. and J.E. Cohen. 1995. Red, white and blue: environmental variance spectra and coexistence in metapopulations. Journal of Theoretical Biology: 176:301316.

Caswell H. and M.G. Neubert. 1998. Chaos and closure terms in plankton food chain models. Journal of Plankton research, 20:1837-1845.

Connell, J.H. 1985. The consequences of variation in initial settlement vs. postsettlement mortality in rocky intertidal communities. Journal of Experimental $\mathrm{Ma}$ rine Biology and Ecology, 93:11-45.

Connell J.H., and R.O. Slatyer. 1977. Mechanisms of succession in natural communities and their roles in community stability and organization. Am. Nat., 111:11191144 .

Cornell, H.V. and R.H. Karlson. 1997. Species richness of reef-building corals determined by local and regional processes. Journal of Animal Ecology, 65:233-241.

Cox, D.R. 1970. The analysis of binary data. Methuen,London.

Denley, E.J. and A.J. Underwood. 1979. Experiments on factors influencing settlement, survival, and growth of two species of barnacles in New South Wales. Journal of Experimental Marine Biology and Ecology, 36:269-293.

Duggins, D.O. 1983. Starfish predation and the creation of mosaic patterns in a kelp dominated community. Ecology, 64:1610-1619.

Fagan, W.F. and L.E. Hurd. 1994. Hatch density variation of a generalist arthropod predator: population consequences and community impact. Ecology, 75:2022-2032.

Formacion S.P., and S.B. Salia. 1994. Markov chain properties related to temporal dominance changes in a Philippine pelagic fishery. Fish. Res., 19:241-256.

Fowler, N.J. 1981. Competition and coexistence in a North Carolina grassland II. The effects of the experimental removal of species. Journal of Ecology, 69;843-854. 
Gilpin, M.E. 1979. Spiral chaos in a predator-prey model. American Naturalist, 107: 306-308.

Grant, W.S. 1977. High intertidal community organization on a rocky headland in Maine, USA. Marine Biology, 44:15-25.

Grant, W.E., J.H. Matis, and W. Miller. 1988. Forecasting commercial harvest of marine shrimp using a Markov chain model. Ecol. Model., 45:183-193.

Hastings, A. and T. Powell. 1991. Chaos in a three-species food chain. Ecology, 72:896-903.

Hill, M.F., J.D. Witman and H. Caswell. 2000a. A Markov chain model of a rocky subtidal community: succession and species interactions in a complex assemblage. Ecology, submitted.

Hill, M.F., J.D. Witman and H. Caswell. 2000b. The effects of temporal and spatial variation on successional patterns in a rocky subtidal community: Statistical methods, Markov chain models, and Species distributions.

Hodge, S, P. Mitchell and W. Arthur. 1999. Factors affecting the occurrence of facilitative effects in interspecific interactions: an experiment using two species of Drosophila and Aspergillus niger. Oikos, 87:166-174.

Holling, C.S. 1973. Resilience and stability of ecological systems. Annual $R$ of Ecology and Systematics, 4:1-23.

Holling, C.S. 1992. Cross-scale morphology, geometry, and dynamics of ecosystems. Ecological Monographs, 62:447-502.

Horn, H.S. 1975. Markovian properties of forest succession. pp. 196-211 in M.L. Cody and J.M. Diamond (eds.) Ecology and evolution of communities. Harvard University Press, Cambridge, MA.

Hosmer, D.W. and S. Lemeshow. 1989. Applied Logistic regression. John Wiley \& 
Sons, New York.

Hughes, T.P. 1994. Catastrophes, phase shifts, and large-scale degradation of a Caribbean coral reef. Science, 265:1547-1551.

Iosifescu M. 1980. Finite Markov processes and their applications. Wiley, New York.

Isagi Y., and N. Nakagoshi. 1990. A Markov approach for describing post-fire succession of vegetation. Ecol. Res., 5:163-171.

Kokkoris, G.D., A.Y. Troumbis, and J.H. Lawton. 1999. Patterns of species interaction strength in assembled theoretical competition communities. Ecology Letters, 2:70-74.

Kot, M., G.S. Sayler, and T.M. Schultz. 1992. Complex dynamics in a model microbial system. Bulletin of Mathematical Ecology, 54:619-648.

Lawton, J.H. 1992. Feeble links in food webs. Nature, 355:19-20.

Leichter, J.J. and J.D. Witman. 1997. Water flow over subtidal rock walls: relationship to distribution and growth rate of sessile suspension feeders in the Gulf of Maine. Journal of Experimental Marine Biology and Ecology, 209:293-307.

Levin, S.A. 1976. Population dynamic models in heterogeneous environments. $A n$ nual review of Ecology and Systematics, 7:287-310.

Levin, S.A. 1992. The problem of pattern and scale in ecology. Ecology, 73:1943-1967.

Levine, J.M. 1999. Indirect facilitation: Evidence and predictions from a riparian community. Ecology, 80: 1762-1769.

Mahdi, A, R. Law and A.J. Willis. 1989. Large niche overlaps among coexisting plant species in a limestone grassland community. Journal of Ecology, 77:386-400.

Masaki, T., W. Suzuki, K. Niiyama, S. Iida, H. Tanaka, and T. Nakashizuka. 1992. Community structure of a species-rich temperate forest, Ogawa Forest Reserve, 
central Japan. Vegetatio, 98:97-111.

May, R. 1973. Stability and complexity in model ecosystems. Monographs in population biology. Princeton University Press, Princeton, New Jersey.

May, R. 1977. Thresholds and breakpoints in ecosystems with a multiplicity of stable states. Science, 269:471-477.

McCann, K., A. Hastings and G.R. Huxel. 1998. Weak trophic interactions and the balance of nature. Nature, 395: 794-798.

McShane, P.E. 1991. Density-dependent mortality of recruits of the abalone Haliotis rubra (Mollusca: Gastropoda). Marine Biology, 110:385-389.

Menge, B.A. and T.M. Farrell. 1989. Community structure and interaction webs in shallow marine hard-bottom communities: tests of an environmental stress model Advances in Ecological Research, 19:189-262.

Neil, W. 1974. the community matrix and interdependence of the competition coefficients. American Naturalist, 108:399-408.

Paine, R.T. 1992. Food-web analysis through field measurement of per capita interaction strength. Nature, 355:73-75.

Pascual, M., and Levine, S.A. 1999. From individuals to population densities: searching for the intermediate scale of nontrivial determinism. Ecology, 80:2225-2236.

Planes. S., J.Y. Jouvenel and P. Lenfant. 1998. Density dependence in postrecruitment processes of juvenile sparids in the littoral of the Mediterranean Sea. Oikos, 83:293-300.

Polis, G.A. 1991. Complex trophic interactions in deserts: An empirical critique of food-web theory. American Naturalist,138:123-155.

Raimondi P.T. 1990. Patterns, mechanisms, consequences of variability in settlement and recruitment of an intertidal barnacle. Oecologia, 85:349-360. 
Raffaelli, D.G. and S.J. Hall. 1966. Assessing the relative importance of trophic links in food webs. in G. Polis and K. Winemiller, eds. Food weds: integration of pattern and dynamics. Chapmen and Hall, New York.

Rego, F., Pereira, J. and Trabaud, L. 1993. Modeling community dynamics of a Quercus coccifera L. garrigue in relation to fire using Markov chains. Ecological Modeling, 66:251-260.

Russ, G.R. 1982. Overgrowth in a marine epifaunal community: competition hierarchies and competitive networks. Oecologia, 53:12-19.

Salia, S.B., and K. Erzini. 1987. Empirical approach to multispecies stock assessments. Trans. Am. Fish. Soc., 116:601-611.

Scheibling, R.E. and A.W. Hennigar 1997. Recurrent outbreaks of disease in sea urchins Strongylocentrotus droebachiensis in Nova Scotia: evidence for a link with large-scale meterologic and oceanographic events. Marine Ecology Progress Series, 152:155-165.

Schoener, T.W. 1983. Field experiments on interspecific competition. American Naturalist, 122:240-285.

Sebens K.P. 1985. The ecology of the rocky subtidal zone. American Scientist, 73:548-557.

Sebens K.P. 1986. Spatial relationships among encrusting marine organisms in the New England subtidal zone. Ecological Monographs, 56:73-96.

Sutherland, J.P. 1974. Multiple stable points in natural communities. American Naturalist, 108:859-873.

Tanner J.E., T.P. Hughes and J.H. Connell. 1994. Species coexistence, keystone species, and succession: a sensitivity analysis. Ecology, 75:2204-2219.

Tanner J.E:, T.P. Hughes and J.H. Connell. 1996. The role of history in community 
dynamics: a modeling approach. Ecology, 77:108-117.

Usher, M.B. 1979. Markovian approaches to ecological succession. Journal of Animal Ecology 48:413-426.

Waggoner P.E., and G.R. Stephens. 1970. Transition probabilities for a forest. $\mathrm{Na}$ ture, 255:1160-1161.

Weiss, C.M. 1948. Seasonal and annual variations in the attachment and survival of barnacle cyprids. Biological Bulletin, 94:236-243.

Wilbur, H.M. 1972. Competition, predation, and the structure of the ambystomaRana sylvatica community. Ecology, 57:3-21.

Wilbur H.M. and J.E. Fauth. 1990. Experimental aquatic food webs: interactions between two predators and two prey. American Naturalist, 135:176-204.

Witman J.D. 1985. Refuges, biological disturbance, and rocky subtidal community structure in New England. Ecological Monographs, 55:421-445.

Witman J.D. 1987. Subtidal coexistence: storms, grazing mutualism, and the zonation of kelp and mussels. Ecological Monographs, 57:167-187.

Witman J.D. 1996. Dynamics of Gulf of Maine benthic communities. pp 51-69 in D. Dow and E. Braasch (eds.) The health of the Gulf of Maine ecosystem: cumulative impacts of multiple stressors. RARGOM Report 96-1, Hanover, NH.

Witman, J.D. and K.P. Sebens. 1988. Benthic community structure at a subtidal rock pinnacle in the central Gulf of Maine. In I. Babb and M. DeLuca (eds.), Benthic Productivity and Marine resources of the Gulf of Maine. National Undersea Research Program report, 88:67-104.

Witman, J.D. and P.K. Dayton. 2000. Rocky subtidal communities. In Bertness, M.D. and M. Hay eds. Marine Benthic Ecology. Sinauer Press (in press)

Wooten, J.T. 1993. Indirect effects and habitat use in an intertidal community: 
interaction chains and interaction modifications. American Naturalist, 141:71-89.

Wooten, J.T. 1997. Estimates and tests of per capita interaction strength: diet, abundance, and impact of intertidally foraging birds. Ecological Monographs, 67:4564 .

Wootton J.T. 2000. Effects of extinction in complex ecosystems: predictions from Markov models. Ecology submitted.

Zajac R.N., R.B. Whitlatch and R.W. Osman. 1989. Effects of interspecific density and food supply on survivorship and growth of newly settled benthos. Marine Ecological Progress Series, 127:149-155. 


\section{Appendix}

The tables below show the rank ordering of species interaction strengths for each of the probability functions $a_{i j}(\mathbf{x})$ pertaining to the best-fit model (Neighborhood Size $=600 \mathrm{~cm}^{2}$ ). Each row in the tables identifies the species whose densities affect the transition from state $j$ to state $i$. The order of the species in the rows gives the species ranking, from strongest to weakest, according to their affect on the probability that a patch in state $j$ changes to state $i$ in one time interval (see section 6.4.3 for details). The species in the columns designated as "Out" are not included in the probability functions $a_{i j}(\mathbf{x})$.

\begin{tabular}{l|ccccccc|c}
\hline \hline & \multicolumn{7}{|c|}{ From HYM } & \\
To & 1 & 2 & 3 & 4 & 5 & 6 & 7 & Out \\
\hline HYM & MYC & SEA & FG & HYM & MYX & BR & CRI & FIL \\
MYX & MYX & MYC & BR & FIL & FG & SEA & CRI & HYM \\
MYC & MYC & HYM & FG & SEA & BR & MYX & CRI & FIL \\
SEA & FIL & MYX & HYM & SEA & BR & MYC & FG & CRI \\
CRI & FG & MYC & MYX & HYM & FIL & BR & SEA & CRI \\
FIL & FIL & SEA & FG & HYM & MYC & MYX & CRI & BR \\
FG & BR & FIL & CRI & HYM & MYC & FG & SEA & MYX \\
\hline
\end{tabular}

\begin{tabular}{l|cccccc|cc}
\hline \hline & \multicolumn{7}{|c|}{ From MYX } & \multicolumn{2}{c}{} \\
To & 1 & 2 & 3 & 4 & 5 & 6 & \multicolumn{2}{c}{ Out } \\
\hline HYM & HYM & MYC & MYX & BR & FG & CRI & FIL & SEA \\
MYX & HYM & CRI & FG & BR & MYC & MYX & FIL & SEA \\
MYC & MYC & FIL & FG & MYX & BR & HYM & CRI & SEA \\
SEA & MYX & HYM & SEA & FG & MYC & BR & FIL & CRI \\
CRI & CRI & MYC & FIL & MYX & BR & FG & SEA & HYM \\
FIL & FIL & SEA & CRI & BR & MYC & MYX & FG & HYM \\
FG & FIL & CRI & HYM & FG & SEA & MYX & MYC & BR \\
\hline
\end{tabular}




\begin{tabular}{l|ccccccc|c}
\hline \hline & \multicolumn{7}{|c|}{ From MYC } \\
To & 1 & 2 & 3 & 4 & 5 & 6 & 7 & Out \\
\hline HYM & FG & MYC & MYX & HYM & CRI & BR & SEA & FIL \\
MYX & MYC & HYM & MYX & BR & FIL & SEA & FG & CRI \\
MYC & FIL & CRI & FG & SEA & HYM & MYC & BR & MYX \\
SEA & SEA & FG & HYM & FIL & CRI & BR & MYX & MYC \\
CRI & FG & CRI & FIL & HYM & MYX & MYC & BR & SEA \\
FIL & FIL & CRI & SEA & HYM & FG & BR & MYC & MYX \\
FG & FG & MYX & BR & CRI & SEA & MYC & FIL & HYM \\
\hline
\end{tabular}

\begin{tabular}{l|ccccccc|c}
\hline \hline & \multicolumn{7}{|c|}{ From SEA } \\
To & 1 & 2 & 3 & 4 & 5 & 6 & 7 & Out \\
\hline HYM & MYX & SEA & BR & FIL & MYC & FG & CRI & HYM \\
MYX & MYX & SEA & BR & HYM & CRI & FG & FIL & MYC \\
MYC & HYM & FG & BR & CRI & SEA & MYX & FIL & MYC \\
SEA & MYX & BR & FIL & SEA & FG & HYM & CRI & MYC \\
CRI & SEA & MYC & FG & MYX & FIL & CRI & BR & HYM \\
FIL & FIL & HYM & MYC & BR & CRI & MYX & FG & SEA \\
FG & BR & FIL & CRI & MYC & MYX & SEA & FG & HYM \\
\hline
\end{tabular}

\begin{tabular}{l|ccccccc|c}
\hline \hline & \multicolumn{7}{|c|}{ From CRI } & \\
To & 1 & 2 & 3 & 4 & 5 & 6 & 7 & Out \\
\hline HYM & HYM & FG & FIL & CRI & SEA & BR & MYX & MYC \\
MYX & MYX & FG & HYM & FIL & BR & MYC & SEA & CRI \\
MYC & MYC & CRI & FIL & BR & HYM & MYX & FG & SEA \\
SEA & FIL & HYM & MYC & SEA & FG & MYX & BR & CRI \\
CRI & FG & CRI & MYX & FIL & BR & HYM & MYC & SEA \\
FIL & FIL & FG & MYX & SEA & MYC & BR & HYM & CRI \\
FG & CRI & FG & HYM & MYX & SEA & BR & FIL & MYC \\
\hline
\end{tabular}




\begin{tabular}{l|ccccccc|c}
\hline \hline & \multicolumn{7}{|c|}{ From FIL } & \\
To & 1 & 2 & 3 & 4 & 5 & 6 & 7 & Out \\
\hline HYM & HYM & MYX & BR & SEA & FIL & MYC & CRI & FG \\
MYX & MYX & SEA & FIL & BR & MYC & CRI & FG & HYM \\
MYC & SEA & FG & MYX & FIL & CRI & HYM & MYC & BR \\
SEA & BR & SEA & CRI & HYM & MYC & MYX & FG & FIL \\
CRI & CRI & FIL & FG & BR & MYX & SEA & MYC & HYM \\
FIL & FIL & BR & MYX & CRI & SEA & FG & MYC & HYM \\
FG & FG & BR & CRI & FIL & HYM & MYC & MYX & SEA \\
\hline
\end{tabular}

\begin{tabular}{l|cccccc|cc}
\hline \hline & \multicolumn{7}{|c|}{ From FG } & \multicolumn{2}{c}{} \\
To & 1 & 2 & 3 & 4 & 5 & 6 & \multicolumn{2}{c}{ Out } \\
\hline HYM & FG & HYM & BR & SEA & FIL & MYC & MYX & CRI \\
MYX & MYX & HYM & BR & FG & SEA & FIL & CRI & MYC \\
MYC & HYM & BR & SEA & CRI & FIL & MYX & FG & MYC \\
SEA & FG & MYX & FIL & BR & HYM & SEA & MYC & CRI \\
CRI & CRI & FG & FIL & MYX & HYM & MYC & SEA & BR \\
FIL & FIL & BR & MYX & FG & HYM & SEA & MYC & CRI \\
FG & CRI & FIL & BR & FG & MYC & SEA & HYM & MYX \\
\hline
\end{tabular}

\begin{tabular}{l|cccccc|cc}
\hline \hline & \multicolumn{7}{|c|}{ From BR } & \multicolumn{2}{|c}{} \\
To & 1 & 2 & 3 & 4 & 5 & 6 & \multicolumn{2}{c}{ Out } \\
\hline HYM & SEA & FIL & MYX & FG & BR & HYM & MYC & CRI \\
MYX & MYX & CRI & HYM & FIL & SEA & FG & BR & MYC \\
MYC & MYC & FG & CRI & FIL & SEA & MYX & HYM & BR \\
SEA & SEA & HYM & MYC & FIL & BR & FG & MYX & CRI \\
CRI & FG & CRI & HYM & MYX & SEA & FIL & MYC & BR \\
FIL & FIL & MYX & HYM & SEA & CRI & FG & MYC & BR \\
FG & FIL & CRI & HYM & MYX & SEA & FG & BR & MYC \\
\hline
\end{tabular}




\section{Chapter 7}

\section{Conclusion and Future Directions}

In this thesis I have presented a series of spatial models describing the dynamics of metapopulations and a rocky subtidal community. These models are motivated by recent theoretical and empirical evidence that the distribution of organisms in space can have important consequences for the function and structure of terrestrial and aquatic systems (Levin and Paine 1974; Steel 1978; Pickett and White 1985; Caswell and Cohen 1991a,b; Weins et. al. 1993; Wu and Levin 1994; Wu and Loucks 1995; Hanski 1999).

While the questions addressed in each of the chapters are diverse, the underlying assumption of the models is that an assemblage of organisms can be represented as a mosaic landscape of discrete patches. Patches can be in one of $N$ possible states, where the state of a patch is defined by the organism or organisms occupying it. In the metapopulation models (Chapters 2 and 3) patches represent habitat fragments capable of supporting a local population. In subtidal community models (Chapters 4,5 , and 6) a patch represents the spatial location of an invertebrate species on the rock wall substrate. In either case, however, the models are formulated in terms of a transition matrix whose $a_{i j}$ entry gives the probability that a patch in state $j$ changes to state $i(i=1, \ldots, N)$ in a single time step. 


\subsection{New insights into the effects of habitat de- struction}

In the past ten to fifteen years, metapopulation models have become an important tool for studying the effects of habitat destruction on metapopulation persistence. While modeling methods have varied, the general thrust of these studies has been to predict how habitat destruction will affect occupied patch frequencies and to quantify the amount of habitat destruction the population can tolerate before it goes extinct (the so called extinction threshold; Tilman et al. 1994). Today the identification of extinction thresholds for endangered species has become an important conservation strategy.

Metapopulation models have typically been predicated on three assumptions

1. The landscape is composed of an infinite number of habitat patches.

2. Propagules are capable of dispersing throughout the landscape; i.e. colonization of an empty patch is not affected by distance.

3. Suitable patches are randomly distributed in space.

Extinction thresholds on fractal landscapes: Chapter 2 looks at the effect of relaxing assumptions 2 and 3 , by exploring the effects of habitat destruction in a spatially explicit cellular automaton (CA) model. In the CA, the dispersal range of propagules is limited to a 3-patch radius and the habitat destruction pattern is explicitly defined using fractal maps. The general findings of this study are illustrated in Figure 7.1. When dispersal is local, the equilibrium frequency of occupied patches $(\hat{p})$ decreases and the extinction threshold $\left(h_{c}\right)$ increases as the fractal dimension $(D)$ of the habitat destruction pattern increases. The take home message is that in large landscapes (consisting of 100's if not 1000's of patches), the spatial structure of suitable habitat is at least as important as the amount of suitable habitat. 


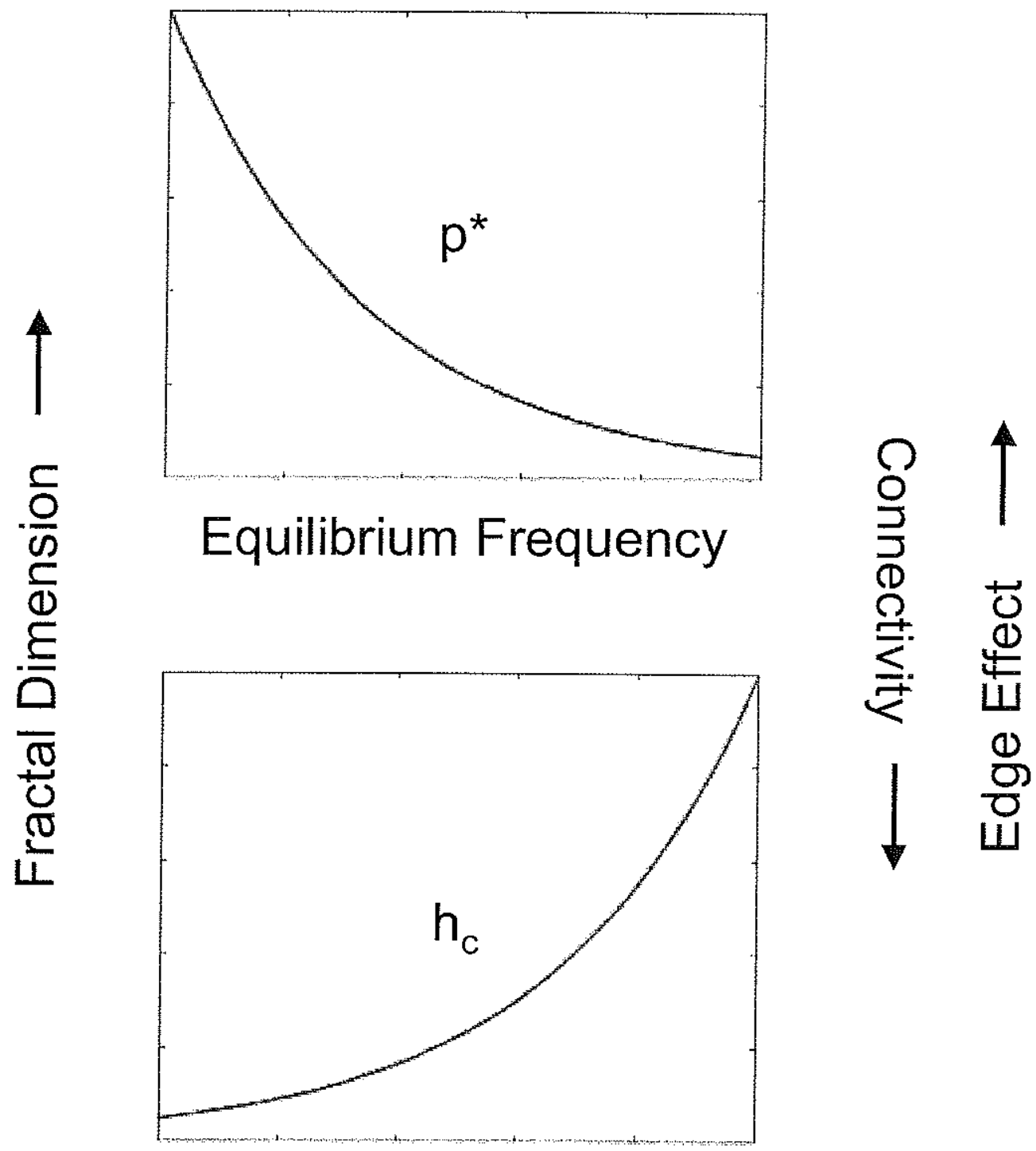

\section{Extinction Threshold}

Figure 7.1: Generalized plots of the equilibrium frequency $\hat{p}$ and the extinction threshold $h_{c}$ as a function of the fractal dimension of the landscape. The arrows on the right and left of the graphs show that decreasing the fractal dimension of the landscape is equivalent to increasing the connectivity of suitable patches and decreasing the amount to edge between suitable and unsuitable habitat. 
In today's modern world, habitat loss is inevitable. While the situation is grim for many endangered species, efforts should at least be made to ensure that habitat destruction patterns have the lowest possible fractal dimension. The fractal dimension of a landscape can be easily measured (Krummel et. al. 1987; Sole and Manrubia 1995; Milne 1988) and conservation efforts to minimize $D$ will maximize connectivity in the remaining suitable territory while minimizing edge effects (Fig. 7.1). Lowering the fractal dimension of habitat destruction patterns may at least give some endangered species a fighting chance. Perhaps this is the best we can hope for in a globalize economy bent on extracting resources at an ever increasing pace.

Chain-Binomial Metapopulation Model: Chapter 3 looks at the effects of relaxing assumption 1, by exploring the effects of habitat destruction in finite patch networks. The chain-binomial metapopulation (CBM) model develop to describe this situation is applicable to marine populations that produce planktonic larvae capable of long distance dispersal. In finite landscapes there is no such thing as a positive equilibrium frequency or extinction threshold, because with probability 1 the metapopulation always goes extinct. The important problem for such systems is to determine how habitat destruction affects the extinction time, $\tilde{\tau}$, of the metapopulation. The CBM model reveals a dangerous scenario regarding attempts to predict the effects of habitat destruction $-\bar{\tau}$ declines greater than exponentially as the number of suitable patches, $S$, declines. Thus, a small amount of habitat destruction has the potential to drastically reduce $\bar{\tau}$ from effectively infinite values (on an ecological time scale) to very small values, leaving a once seemingly healthy population on the verge of extinction. The sensitivity of $\bar{\tau}$ to changes in $S$ makes the task of identifying critical habitat thresholds in finite landscapes a near impossibility. This result has especially grave consequences for endangered species, who by definition are confined to small patch networks. 


\subsection{Markov chain models of sessile communities}

The structure of marine communities is an emergent property of species interactions occurring over relatively small spatial scales and physical processes occurring over a range of spatial scales. Determining how these processes interact to produce complex community structures, however, remains a fundamental problem in marine ecology.

In the second half of this thesis, I have shown that Markov chains have the potential to vastly improve our ability to identify key ecological factors (succession, disturbance, competition, environmental variability, etc.) and key species that are important for the structure and function of marine sessile communities. The approach I have taken is to start with simple Markov chain models, which exclude much of the detail of the system, before proceeding to more complex models. Even the most complicated of them, however, is not intended as a detailed quantitative description of all the factors affecting rocky subtidal communities. My philosophy is that comparing related models that differ in biological details is a more powerful approach for understanding community dynamics than analyzing any one model.

Linear Markov chain models: In chapter 4 I began the analyses of the rocky subtidal community by constructing a linear time-invariant Markov chain. This is the simplest model that incorporates the basics of patch transitions, species replacement, and disturbance. While the linear Markov chain ignores the effects of environmental variability and density dependence, it accurately predicted species distribution in the Gulf of Maine, provided ecological information for categorizing species into functional groups, and revealed that $38 \%$ of the substrate was occupied by a different species each year.

One of the biggest advantages of linear Markov chains is that sensitivity analysis can be developed to determine how changes in model parameters affect predicted community patterns. Sensitivity analysis has already become an essential part of demographic analysis (Tuljapurkar and Caswell 1997, Caswell 2000), and has the potential 
to become equally valuable in community modeling. Here I used sensitivity analysis to characterize how changes in elements of the transition matrix affect predictions of species abundance and community diversity. The results suggest that diversity is much more sensitive to changes in colonization probabilities than to species replacement probabilities. This finding is consistent with observations by Witman and Sebens (1990), who found that competition plays a relatively minor role in structuring subtidal communities.

Linear Markov chain models are also ideal for quantifying the effects of species removal on diversity and community resilience. Analysis of the subtidal transition matrix showed that the removal of Hymedsmia spp. 1 or Myxilla fimbriata produces the largest change in diversity, however, the overall change is relatively small. This finding indicates that there are no real keystone species in the subtidal community. The removal of bare rock or Crisia eburnea, however, produced relatively large changes in the rate of converge to equilibrium, suggesting that these states are important for community resilience. The removal analyses developed in chapter 4 are Markov chain analogs to species removal experiments in natural systems. They provide a means of classifying the relative importance of individual species to the structure and stability of the community when experimental manipulations are not possible.

Environmental variability: The next step in the complexity hierarchy (chapter 5) was to test for significant effects of temporal and spatial variation on patch transition probabilities. Because this is a 2-factorial test, I used log-linear analysis, which showed that transition probabilities varied significantly between quadrat locations and between years in the subtidal zone. While some authors (e.g. Li 1996, Childress et al. 1998) have argued that such variability must be taken into account for Markov chains to adequately predict equilibrium community structure, I found that including this variability in the subtidal model had little effect on predicted species abundances. On the other hand, simulation of the time-varying Markov chain shows that temporal 
variation in successional processes (i.e. transition probabilities) has important consequences for transient dynamics. In the subtidal community the time-varying model predicts frequent shifts in composition between a Hymedesmia dominated community and a Crisia dominated community. This pattern is consistent with observations in the Gulf of Maine over the past ten years (Witman 1996).

Chapter 5 also introduces a method for comparing probability matrices (Cohen et al. 1998) and uses it to characterize regional variability in the distribution of species in space and time. While this methodology is somewhat of a digression from the Markov chain approach, it provides a simple but effective way of characterizing the spatial and temporal stability of individual species relative to other organisms in the community. In the subtidal community, Cohen's method predicts there is more variability between quadrats in the temporal distribution of species, than there is over time in the spatial distribution of species. This result is consistent with the log-linear analysis, which shows a greater effect of space on transition probabilities than time.

Density dependence Chapter 6 increases the complexity in the subtidal models further by incorporating the effects of density dependence on transition probabilities. The most important contribution of this work is the development of maximum likelihood methods to estimate the effects of species densities on transition probabilities. The likelihood methods will allow ecologist to estimate density-dependent interactions in an unmanipulated community from spatial time series data. By focusing on patch transition probabilities, instead of individual species, the strength of interactions among all species can be estimated simultaneously. Any indirect effects are automatically incorporated into the model because species interactions are estimated by taking into account the dynamics of the community as a whole. This is not true for species removal experiments, which have traditionally been used to measure species interaction strengths in community assemblages (Paine 1992). 


\subsection{Future research directions}

Below I briefly describe some future research directions related to the modeling methods presented in this thesis.

\subsubsection{Metapopulations models: Habitat restoration and Ma- rine reserves}

Habitat restoration: To study the effects of habitat destruction I assumed that once habitat was destroyed it remained destroyed forever. In many marine systems, however, there is a continuous turnover of suitable habitat, in which the destruction of habitat in one region is balanced by the restoration of habitat in another. A classic example is hydrothermal vents (Mullineaux et al. 1991), in which subpopulations among vent sites can be described as a metapopulation living on a geographical expanse of ephemeral patches. Extinction of a subpopulation results when the vent site it occupies becomes inactive (a process equivalent to habitat destruction). Propagules produced by many vent species, however, can disperse over long distances (Lutz 1988) and are capable of colonizing newly developing vent sites (a process equivalent to habitat restoration).

To study the characteristic of such systems I will include habitat restoration in the metapopulation models presented in chapter 2. Figure 7.2 shows an idealize transition diagram for a metapopulation model with habitat restoration. The goal of this research is to analyze the system of equations specific by the transition diagram in order to characterize how the rate of destruction and recovery affect persistence and extinction times. I will then translate this model into a spatially explicit cellular automaton model to study how the spatial location of habitat sites affects the dynamics of the system. 


\section{Suitable habitat}

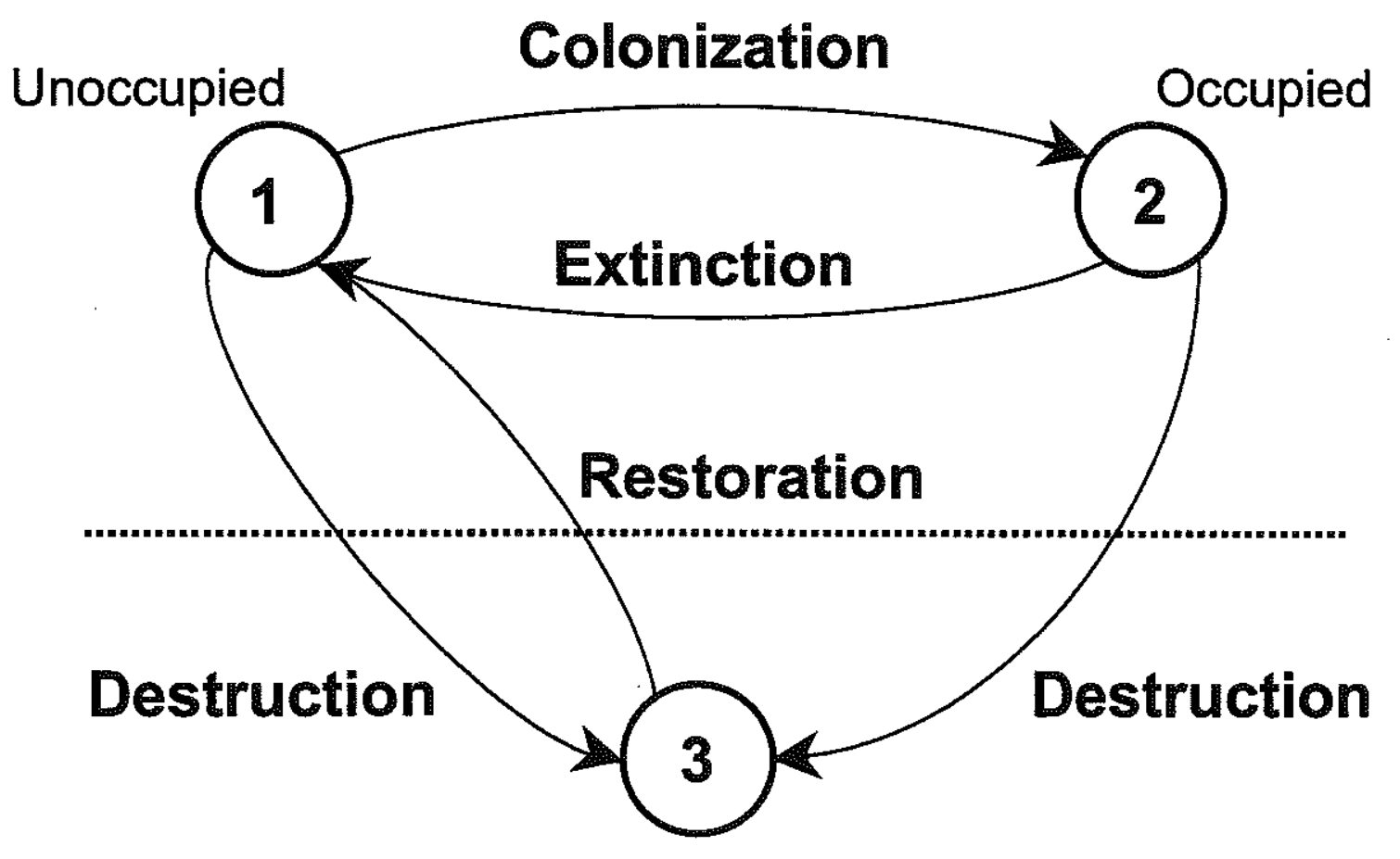

\section{Unsuitable habitat}

Figure 7.2: Generalized transition diagram for a metapopulation model with habitat destruction and habitat restoration 


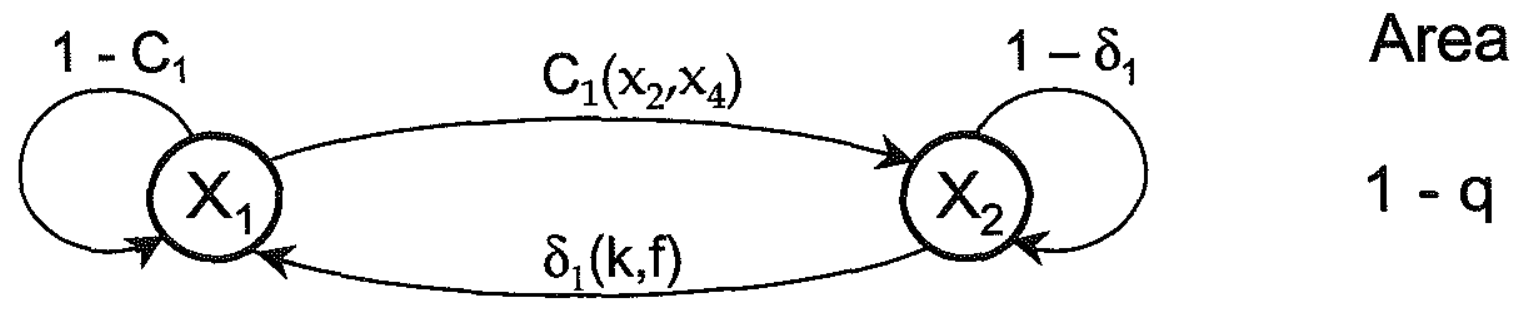

Non-Reserve

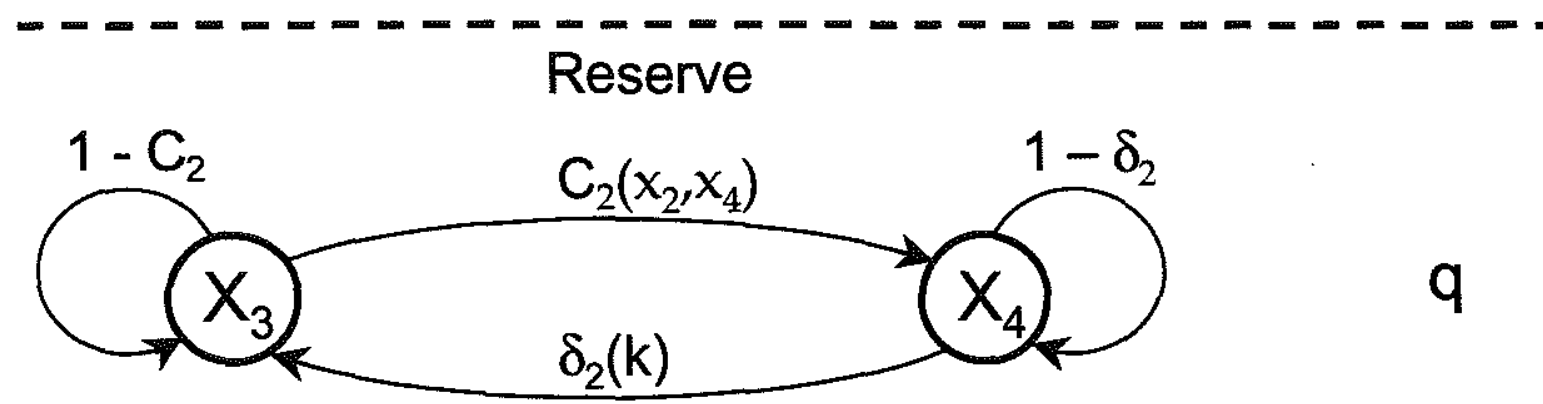

Figure 7.3: Transition diagram for a hyper-population model of a marine reserve. The proportion of the area that falls within the reserve equals $q$, where $0 \leq q \leq 1$. $X_{1}$ and $X_{3}$ represent unoccupied patches in the non-reserve and reserve, respectively. $X_{2}$ and $X_{4}$ represent occupied patches in the non-reserve and reserve. $C_{i}(i=1,2)$ is the probability that an unoccupied patch is colonized. $\delta_{i}(i=1,2)$ is the probability that a local population goes extinct. See text for details.

Marine reserve design: Setting aside reserve areas for protection from fishing is an important option being considered in the management of fisheries (Clark 1996; Fogarty 1999). To study how marine reserves could help conserve fish populations and benefit fisheries I have developed a hyper-population model describing the dynamics of a pair of metapopulations located in two distinct regions. Figure 7.3 shows a transition diagram for the hyper-population model, in which the distinct regions correspond to reserve and non-reserve areas in a fishery. Fishing is only allowed in the non-reserve areas, and the proportion of the fishery that is set aside as non-reserve is $1-q(0 \leq q \leq 1)$.

Metapopulations in the reserve and non-reserve regions are connected to each 
other via the colonization functions $C_{1}\left(x_{2}, x_{4}\right)$ and $C_{2}\left(x_{2}, x_{4}\right)$, given by the equations

$$
\begin{aligned}
& C_{1}\left(x_{2}, x_{4}\right)=1-\exp \left(\frac{b\left(x_{2}\left(1-\alpha_{21}\right)+x_{4} \alpha_{12}\right)}{q}\right) \\
& C_{2}\left(x_{2}, x_{4}\right)=1-\exp \left(\frac{b\left(x_{2} \alpha_{21}+b_{2} x_{4}\left(1-\alpha_{12}\right)\right)}{1-q}\right)
\end{aligned}
$$

where $b$ is the reproductive parameter (see Eq. 2.10), $x_{i}(i=2,4)$ is the proportion of occupied patches in the fishery, $\alpha_{12}$ is the proportion of propagules produced in the reserve that end up in the non-reserve $\left(0 \leq \alpha_{12} \leq q\right)$, and $\alpha_{21}$ is the proportion of propagules produced in the non-reserve that end up in the reserve $\left(0 \leq \alpha_{21} \leq 1-q\right)$. Thus, the parameters $\alpha_{12}$ and $\alpha_{21}$ define the level of mixing of propagules between reserve and non-reserve areas.

Fishing mortality in the non-reserve areas increases the disturbance rate of occupied patches and the total fishing pressure is assumed to be constant (i.e. fisherman don't stop fishing just because some of the fishing grounds are set aside as reserves). This means that the fishing pressure per unit area increases as $q$ increases. This effect can be modeled as

$$
\begin{aligned}
& \delta_{1}=1-\exp -\left(k+\frac{f}{1-q}\right) \\
& \delta_{2}=1-\exp (k)
\end{aligned}
$$

where $k$ is a disturbance parameter and $f$ is a measure of the total fishing pressure.

The marine reserve metapopulation model is applicable to benthic species, such as scallops and oysters, as well as coral many reef fish (Man et al. 1995). I propose to analysis this model to determine the optimal reserve size for maximizing the exploitable stock and the sustainable yield of the fishery. I will study how this optimum varies as a function $b, k, f, \alpha_{12}$ and $\alpha_{21}$. I will also translate the reserve-model into a spatially explicit cellular automaton to look at the effect of the spatial arrangement of reserve areas on stock size and maximum yield. 


\subsubsection{Spatially explicit models of benthic communities}

Spatial-Dependent Markov Chain Models: In this project I will develop a spatially explicit model of the subtidal community by identifying patch transition probabilities that are dependent on neighboring species densities. This approach is similar to the methods I used in chapter 6 except that the likelihood functions must estimate species interaction effects on a patch-by-patch basis. Thus, each cell might have its own vector of independent variables, and the parameters would be estimated over the entire set of observed transitions. This approach will be computationally intensive, but it is worth exploring because it may give different results from the previous methods, which only approximated spatial-dependent effects by dividing the quadrats into smaller subunits.

I will use the information from this analysis to build a cellular automaton (CA) model in which patches transitions are a function of the states of neighboring patches. There is wide latitude in the form of the neighborhood and the nature of the transition functions. The difference between a CA model and the nonlinear Markov chain model is that the explicit spatial arrangement will affect the outcome. I will use the CA model to explore these effects on coexistence and spatial pattern, and explore the possibility of long-term phase shifts in community composition.

A Mechanistic Cellular Automaton Model: State transitions are actually determined by mechanisms that involve growth, recruitment, disturbance, and competition for space. Here I propose to develop a cellular automaton model based on measurements of these mechanisms. My goal is to produce a matrix that will give the probabilities of transitions among states as a function of the state of the neighboring patches.

The procedure will be as follows. 
1. First, I will estimate the probabilities of each of the basic interactions. My goal is to obtain estimates that give, as nearly as possible, the probabilities of each process in the absence of the others.

(a) I will estimate the probability that an empty patch is colonized by each species from a set of species removal experiments (Witman, unpublished data). These experiments were performed by clearing $30 \times 20 \mathrm{~cm}^{2}$ areas of all organisms and following recolonization over a two year period.

(b) I will estimate probabilities of growth into empty patches from the photographic records of quadrats over time. This will require some ingenuity, because growth rates (which are directly measurable) depend on the amount of available space and the species present in neighboring patches.

(c) I will estimate probabilities of competitive overgrowth by examining locations where two species occupy adjacent patches.

(d) I will estimate probabilities of disturbance by monitoring the appearance of empty patches. I will use the method of Caswell and Etter (1993) to model disturbances of different sizes.

2. The different processes by which a patch can change state are a set of competing risks (e.g., Chiang 1966, David and Moeschberger 1978). I will use competing risk theory to calculate the probabilities of changing state in the presence of competing risks. These probabilities will form the cellular automaton transition matrix.

I will study the model by simulation, in order to determine how changes in recruitment, growth, and competitive exclusion rates affect community structure. I will also investigate the impact of the frequency and size distribution of disturbance events on model behavior. Finally, I will compare predictions of the mechanistic CA with the non-mechanistic models in order to determine which method better characterizes the dynamics of the rocky subtidal community. 


\subsection{References}

Caswell H. 2000. Matrix population models. Construction, analysis, and interpretation. Sinauer Associates Inc., Sunderland, MA. 2nd Ed., in press.

Caswell, H. and J. E. Cohen. 1991a. Communities in patchy environments: a model of disturbance, competition, and heterogeneity. In Ecological Heterogeneity, J. Kolasa (ed.). Springer-Verlag, Berlin.

Caswell, H. and J. E. Cohen. 1991b. Disturbance and diversity in metapopulations. Biological Journal of the Linnaean Society, 42:193-218.

Caswell H., and R.J. Etter. 1993. Ecological interactions in patchy environments: From patch-occupancy models to cellular automata, in J.H. Steele and S. Levon (eds.) Patch dynamics. Springer-Verlag, New York.

Chiang, C.L. 1968. Introduction to stochastic processes in biostatistics. Wiley, New York.

Clark, C. 1996. Marine reserves and the precautionary management of fisheries. Ecological Applications, 6:369-370.

Cohen J.E., J.H.B. Kemperman, and Gh. Zbăganu. 1998. Comparisons of Stochastic Matrices; with applications in Information Theory, Statistics, Economics, and Population Sciences. Birkhäuser, Boston, MA.

Cox, D.R. 1970. The analysis of binary data. Methuen, London.

David, H.A. and M.L. Moeschberger. 1978. The theory of competing risks. Charles Griffin, London.

Fogert, M.J. 1999. Essential habitat, marine reserves and fishery management. Trends in Ecology and Evolution, 14:133-134.

Hanski, I. 1999. Metapopulation ecology. Oxford University Press, Oxford. 
Krummel, J.R., R.H. Gardner, G. Sugihara, R.V. O'Neill and P.R. Coleman. 1987. Landscape patterns in a disturbed environment. Oikos, 48:321-324.

Levin, S.A. and R.T. Paine. 1974. Disturbance, patch formation and community structure. Proceedings of the National Academy of Sciences, 71:2744:2747.

Lutz, R.A.D. 1988. Dispersal of organisms at deep-sea hydrothermal vents: A review. Oceanologica Acta: Hydrothremalism, Biology and Ecology Symposium 1985, pp 2329.

Man, A., R. Law and N.V.C. Polunin. 1995. Role of Marine reserves in recruitment to reef fisheries: a metapopulation model. Biological Conservation, 71:197-204.

Milne, B.T. 1988. Measuring the fractal geometry of landscapes. Applied Mathematics and Computation, 27:67-79.

Mullineaux, L.S., P.H. Wiebe, and E.T. Baker. 1991. Hydrothermal vent plumes: Larval highways in the deep sea? Oceanus, 34:64-68.

Paine, R.T. 1992. Food-web analysis through field measurement of per capita interaction strength. Nature, 355:73-75.

Pickett S.T.A. and P.S. White 1985. Ecology of Natural Disturbance and Patch Dynamics. Academic Press, San Diego.

Sole, R.V. and S.C. Manrubia. 1995. Are rainforests self-organized in a critical state? Journal of Theoretical Biology, 173:31-40.

Steel, J.H. 1978. Spatial Patterns in Plankton Communities. Plenum, New York.

Tillman, D., R.M. May, C.L. Lehman and M.A. Nowak. 1994. Habitat destruction and the extinction debt. Nature, 371:65-66.

Tuljapurkar, S. and H. Caswell. 1997. Structured population models in marine, terrestrial and freshwater systems. Chapman and Hall, New York. 
Weins, J.A., N.C. Stenseth, B. Van Horne and R.A. Ims. 1993. Ecological mechanisms and landscape ecology. Oikos, 66:369-380.

Witman J.D. 1996. Dynamics of Gulf of Maine benthic communities. pp 51-69 in D. Dow and E. Braasch (eds.) The health of the Gulf of Maine ecosystem: cumulative impacts of multiple stressors. RARGOM Report 96-1, Hanover, NH.

Witman, J.D. and K.P. Sebens. 1990. Distribution and ecology of sponges at a subtidal rock ledge in the central Gulf of Maine. PP. 391-396 in K. Rutzler (ed.) New perspectives in sponge biology. Smithsonian Institution Press, Washington, D.C.

Wu, J. and S.A. Levin 1994. A spatial patch dynamic modeling approach to pattern and process in an annual grassland. Ecological Monogrpahs,64:447-464.

Wu, J. and O.L. Loucks. 1995. From balance of nature to hierarchical patch dynamics: a paradigm shift in ecology. The Quarterly Review in Biology, 70:439-466. 